Immunomodulating polysaccharides, triterpenoids and melanin from the medicinal fungus Inonotus obliquus (Chaga)

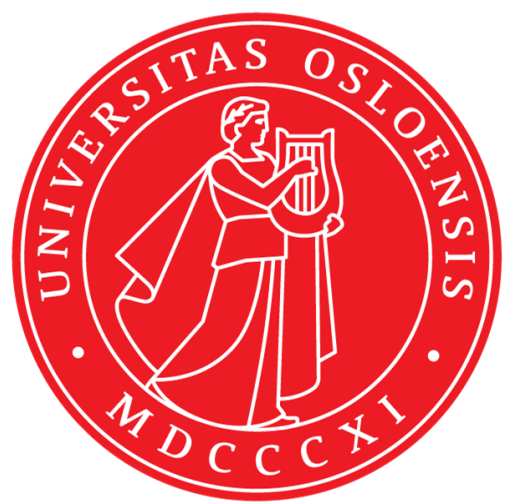

PhD Thesis

Christian Winther Wold

Section for Pharmaceutical Chemistry

Department of Pharmacy

Faculty of Mathematics and Natural Sciences

University of Oslo

Norway

2020 
(C) Christian Winther Wold, 2020

Series of dissertations submitted to the

Faculty of Mathematics and Natural Sciences, University of Oslo No. 2300

ISSN $1501-7710$

All rights reserved. No part of this publication may be reproduced or transmitted, in any form or by any means, without permission.

Cover: Hanne Baadsgaard Utigard.

Print production: Reprosentralen, University of Oslo. 


\section{TABLE OF CONTENTS}

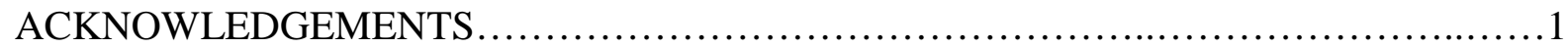

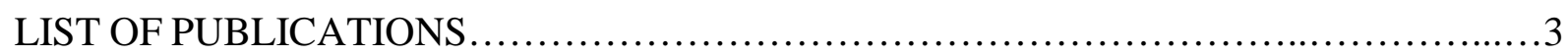

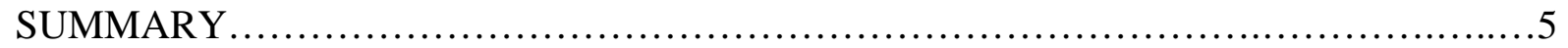

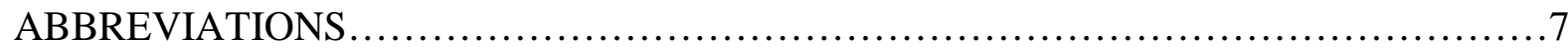

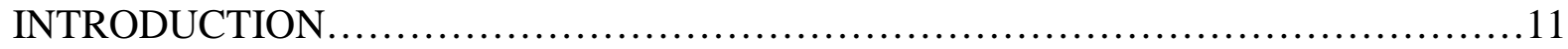

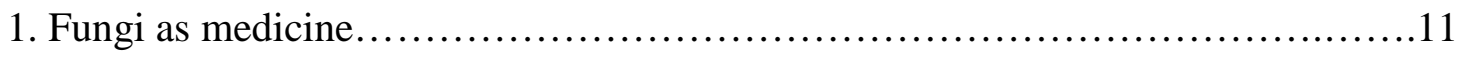

1.1. Inonotus obliquus...............................................12

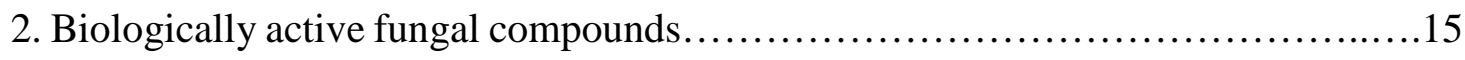

2.1. Polysaccharides................................................ 15

2.2. Triterpenoids.................................................... 18

2.3. Melanin.......................................................... 20

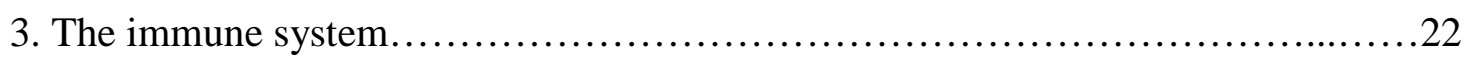

3.1. The complement system......................................... 24

3.2. Macrophages and dendritic cells.................................26

3.2.1. Tumor-associated macrophages...........................28

3.3. Pattern recognition receptors...................................29

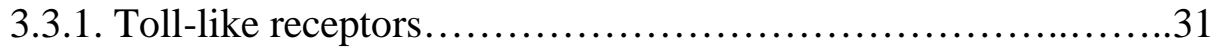

3.3.2. C-type lectin receptors................................. 31

4. Immunomodulating polysaccharides from fungi in cancer therapy................32

AIMS OF THE THESIS ............................................................ 33

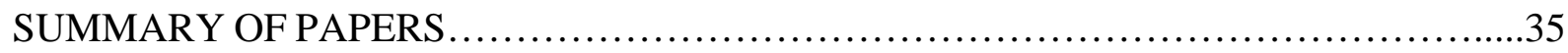

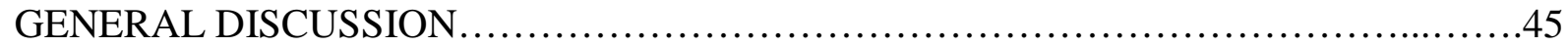

CONCLUDING REMARKS AND FUTURE PERSPECTIVES.........................63

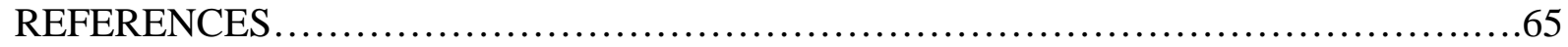

SCIENTIFIC PAPERS I-III................................................... 72 



\section{ACKNOWLEDGEMENTS}

This work was performed from 2015 through 2020 at the section for Pharmaceutical Chemistry, Department of Pharmacy, University of Oslo, Norway. A majority of the biological assays were conducted in the Tumor Immunology lab at the Department of Pathology, Rikshospitalet, Oslo University Hospital. Some of the work was carried out during research stays at the Department of Chemistry, Denmark Technical University and at Scripps Institution of Oceanography, University of California, San Diego.

I am grateful to all the people who have made this thesis possible.

First and most importantly, a big thank you to my main supervisor Kari Inngjerdingen. Without you, this thesis would not have been possible. From the first day of the project, you believed in me - and you made me believe in myself. You allowed me to follow my own path while at the same time making sure I was on the right track throughout the project. You have been an invaluable source of knowledge and a person I always could come to for scientific and nonscientific discussions. You have always been there when I needed support.

I would also like to express my sincerest gratitude to my co-supervisors Helle Wangensteen and Alexandre Corthay. Helle - your deep insight into the world of chemistry has been invaluable, and your enthusiasm and positive energy is contagious. Alex - your immense knowledge of the world of immunology never seizes to amaze me, and I have felt welcome in your group from the first day of the project.

I would like to thank all the people in the Pharmacognosy group and the Tumor Immunology group for making these years interesting, challenging and fun! There are too many people to thank personally, but you all know who you are.

To all my friends - thank you for giving me the proper perspectives in life. A special thanks to Even for making me become interested in Chaga in the first place.

Thank you to my family - my two brothers, mom and dad. Your unconditional love and support in whatever I am doing is and will always be invaluable.

Lastly, I would like to thank my loving girlfriend Caroline for supporting me throughout all these years. You are the most important person in my life, and without you, I would not have been where I am today. 


\section{LIST OF PUBLICATIONS INCLUDED IN THE THESIS}

\section{Paper I}

Structural characterization of bioactive heteropolysaccharides from the medicinal fungus Inonotus obliquus (Chaga)

Christian Winther Wold, Christian Kjeldsen, Alexandre Corthay, Frode Rise, Bjørn E. Christensen, Jens Øllgaard Duus, Kari Tvete Inngjerdingen

Carbohydrate Polymers (2018), 185: 27-40

\section{$\underline{\text { Paper II }}$}

Polysaccharides from the fungus Inonotus obliquus (Chaga) activate macrophages into a tumoricidal phenotype via interaction with TLR2, TLR4 and Dectin-1a

Christian Winther Wold, Panagiotis Christopoulos, Maykel Arias, Inger Øynebråten, Alexandre Corthay, Kari Tvete Inngjerdingen

Manuscript

\section{Paper III}

Bioactive triterpenoids and water-soluble melanin from Inonotus obliquus (Chaga) with immunomodulatory activity

Christian Winther Wold, William H. Gerwick, Helle Wangensteen, Kari Tvete Inngjerdingen Journal of Functional Foods (2020), 71: 104025 


\section{OTHER CO-AUTHORED PAPERS}

Neutral polysaccharide from the leaves of Pseuderanthemum carruthersii: presence of 3$O$-methyl galactose and anti-inflammatory activity in LPS-stimulated RAW 264.7 cells Bac VH, Paulsen BS, Truong LV, Koschella A, Trinh TC, Wold CW, Yogarajah S, Heinze T Polymers (2019), 11 (7): 1219

Immunomodulating polysaccharide complexes and antioxidant metabolites from cyanobacteria

Georgiev YN, Batsalova TG, Dzhambazov BM, Ognyanov MH, Denev PN, Antonova DV, Wold CW, Yanakieva IZ, Teneva I, Paulsen BS, Simova SD

To be submitted to Algal Research

Immunomodulatory polysaccharide complexes from Geranium sanguineum L. and Macrolepiota procera (Scop.) Singer

Georgiev YN, Batsalova TG, Ognyanov MH, Denev PN, Dzhambazov BM, Simova SD, Wold $\underline{\mathrm{CW}}$, Paulsen BS,

To be submitted to International Journal of Biological Macromolecules

Simulated green turtle grazing reduces seagrass productivity and alters benthic community structure while triggering further disturbance by feeding stingrays Cannon AL, Hynes MG, Brandt M, Wold CW, O’Dea A, Altieri A, Smith JE Submitted to Marine Ecology Progress Series

Ethnopharmacology, biological activities, and chemical compounds of Canarium strictum: an important resin-yielding medicinal tree in India Seethapathy GS, Wold CW, Ravikumar K, de Boer HJ, Wangensteen H Manuscript 


\section{SUMMARY}

Inonotus obliquus (Family: Hymenochaetaceae) is a fungus growing on trees from the Betula and Alder genera and is used in traditional medicine in Northern European and Asian countries. The fungus is widely prepared as a decoction in a similar fashion to herbal teas and used both internally and externally, for instance in the treatment of external wounds or against gastrointestinal disorders. Despite its widespread use, the immunological potential of $I$. obliquus has been poorly investigated. The overall aim of this thesis was to isolate and chemically characterize compounds from I. obliquus and to investigate the immunomodulating properties of these compounds.

Polysaccharides were obtained from water- and alkali extracts, and these were purified using column chromatography and characterized using spectroscopic and chromatographic methods, including NMR spectroscopy and GC-MS. The water-soluble polysaccharides were complex and relatively small (10-73 kDa), consisting of several different monosaccharides and types of linkages, such as $(1 \rightarrow 3)$-linked and $(1 \rightarrow 6)$-linked $\beta$-glucose, $(1 \rightarrow 6)$-linked $\alpha$-galactose and $(1 \rightarrow 4)$-linked $\alpha$-galacturonic acid. The alkaline extracted polysaccharides were large $(>500$ $\mathrm{kDa})$, particulate polymers consisting mainly of $(1 \rightarrow 3)$-linked $\beta$-glucose. The acidic, watersoluble polysaccharides IOI-WAcF1 and IOI-WAcF3 were shown to induce the most potent pro-inflammatory effects in bone-marrow derived macrophages in vitro. The activation of the macrophages resulted in increased production of nitric oxide and the pro-inflammatory cytokines IL- 6 and TNF- $\alpha$. Furthermore, macrophages activated with polysaccharides in combination with IFN- $\gamma$ became tumoricidal, as seen by their ability to inhibit cell growth of Lewis lung carcinoma cells in a co-culture model. Using HEK-Blue ${ }^{\mathrm{TM}}$ reporter cell lines, the polysaccharides were shown to act through multiple receptors, including TLR2, TLR4 and Dectin-1a.

Six triterpenoids were obtained from dichloromethane extracts of I. obliquus, isolated by using chromatographic methods including preparative HPLC and TLC, and characterized by NMR spectroscopy and MS. The triterpenoids inotodiol and 3 $\beta$-hydroxy-8,24-dien-21-al were shown to activate the human complement cascade through the classical and lectin pathways. In addition, betulin-3-O-caffeate, isolated from the first time from I. obliquus, displayed antiinflammatory effects by inhibiting LPS-induced NO production in macrophages, while betulinic acid, betulin and inotodiol showed anti-proliferative effects against the lung carcinoma cell line H460 and the colorectal adenocarcinoma cell line HT29-MTX. 
A water-soluble melanin fraction was obtained after repeated steps of water extraction, acidic treatment and dialysis. The pigment was characterized by various methods such as combustion analysis and HPLC analysis of its degradation products, as well as by NMR spectroscopy. The melanin fraction inhibited the human complement cascade though interaction with the classical, lectin and alternative complement pathways. In addition, the melanin fraction showed antiinflammatory effects by inhibiting LPS-induced NO production in macrophages.

Collectively, the results presented in this thesis demonstrate the immunological capacity of compounds from I. obliquus belonging to widely different classes of natural products. The specific immunological activities were shown to be highly dependent on which class of compound that were tested and the experimental setup utilized. This emphasizes the importance in natural product research of testing isolated compounds as well as crude extracts, because different compounds could potentially negate each one's individual effects. In conclusion, $I$. obliquus contains polysaccharides, triterpenoids and melanin pigments with potent immunomodulating activities, some of the compounds being unique to this fungus. These compounds would be worthwhile investigating further, in order to discover the full potential of I. obliquus as a medicinal fungus and as a source for new pharmaceutical drugs. 


\section{ABBREVIATIONS}

3-O-Me-Gal 3-O-methylated galactose

Abbreviation for the alkaline polysaccharide fraction IOI-A1

AcF1 Abbreviation for the acidic polysaccharide fraction IOI-WAcF1

AcF2 Abbreviation for the acidic polysaccharide fraction IOI-WAcF2

AcF3 Abbreviation for the acidic polysaccharide fraction IOI-WAcF3

Ara

Arabinose

BMDM Bone marrow-derived macrophage

CLR C-type lectin receptor

CR3 Complement receptor 3

DAMP Damage-associated molecular patterns

DCM Dichloromethane

DHI 5,6-Dihydroxyindole

DHICA 5,6-Dihydroxyindole-2-carboxylic acid

DHN 1,8-Dihydroxynaphthalene

EtOH Ethanol

EWN Abbreviation for the neutral polysaccharide fraction IOE-WN

Gal Galactose

GalA Galacturonic acid

GC Gas chromatography

GC-MS Gas chromatography - mass spectrometry

Glc Glucose

HMBC Heteronuclear multi-bond correlation

HPLC High-performance liquid chromatography

HSQC Heteronuclear single quantum coherence/correlation 


\begin{tabular}{|c|c|}
\hline IFN- $\gamma$ & Interferon-gamma \\
\hline IL-4 & Interleukin 4 \\
\hline IL-6 & Interleukin 6 \\
\hline IL-17 & Interleukin 17 \\
\hline iNOS & Inducible nitric oxide synthase \\
\hline IOE & Inonotus obliquus exterior part \\
\hline IOE-WN & 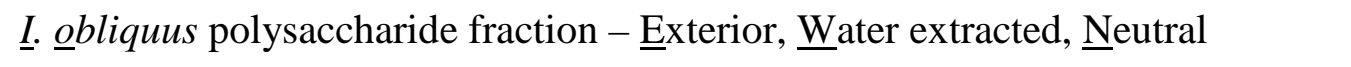 \\
\hline IOI & Inonotus obliquus interior part \\
\hline IOI-A1 & 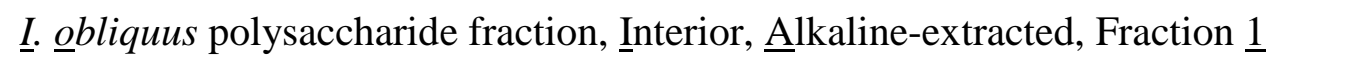 \\
\hline IOI-WAcF1 & 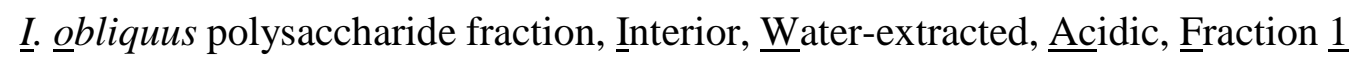 \\
\hline IOI-WAcF2 & 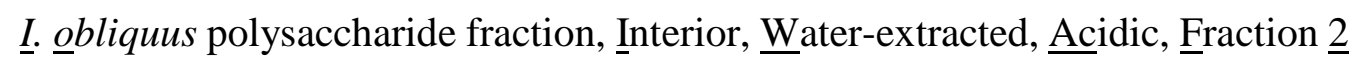 \\
\hline IOI-WAcF3 & 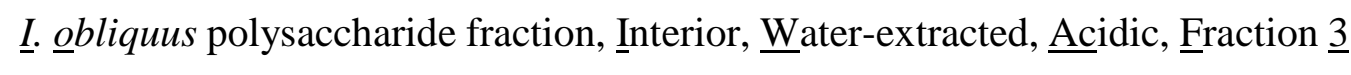 \\
\hline IOI-W & A crude polysaccharide extract obtained after $\mathrm{EtOH}$ precipitation and dialysis. \\
\hline IOI-WN & 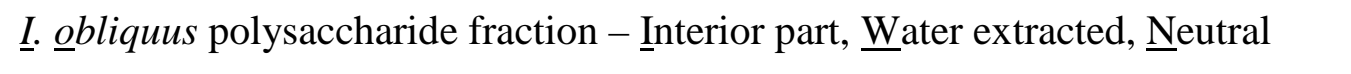 \\
\hline IWN & Abbreviation for the neutral polysaccharide fraction IOI-WN \\
\hline LPS & Lipopolysaccharide \\
\hline Man & Mannose \\
\hline $\mathrm{MeOH}$ & Methanol \\
\hline MAC & Membrane attack complex \\
\hline NK cell & Natural killer cell \\
\hline NLR & NOD-like receptors \\
\hline NMR & Nuclear magnetic resonance \\
\hline $\mathrm{NO}$ & Nitric oxide \\
\hline PAMP & Pathogen-associated molecular patterns \\
\hline
\end{tabular}


PRR Pattern recognition receptors

Rha Rhamnose

RLR RIG-1-like receptors

SEC Size-exclusion chromatography

SEC-MALLS Size-exclusion chromatography, Multi angle laser light scattering

Syk Spleen tyrosine kinase

TAM Tumor-associated macrophages

$\mathrm{T}_{\mathrm{h}} 1$ cell $\quad$ Subset of $\mathrm{T}$ helper cells (producing IFN- $\gamma$ )

$\mathrm{T}_{\mathrm{h}} 2$ cell $\quad$ Subset of $\mathrm{T}$ helper cells (producing IL-4)

$\mathrm{T}_{\mathrm{h}} 17$ cell $\quad$ Subset of $\mathrm{T}$ helper cells (producing IL-17)

TNF- $\alpha \quad$ Tumor necrosis factor alpha

TLC Thin-layer chromatography

TLR Toll-like receptors

Xyl Xylose 


\section{INTRODUCTION}

\section{Fungi as medicine}

Throughout human history, plants and mushrooms have been used as a source of medicine and food in virtually all parts of the world. Even today, nature continues to be important for the pharmaceutical industry, for example as a starting point for drug discovery (1). The fungal kingdom is a vast resource of pharmaceutical compounds, exemplified by antibiotics from Penicillium fungi, the cholesterol-lowering statins and the immunosuppressant cyclosporine. A recent report on fungal diversity estimated that only 3-8 \% of total fungal species in the world have been accounted for, and thus fungi serve as an untapped source of new and exciting compounds to be discovered in years to come $(2,3)$. Most existing fungal-derived drugs come from fungi belonging to the Ascomycota phylum (e.g. molds), but fungi from the Basidiomycota phylum - which includes the edible mushrooms - are getting increased attention from the scientific community (4). Compounds of medicinal interest have been isolated from mushrooms that have had a long history in traditional cuisine and medicine, such as Ganoderma lucidum (Reishi/ Lingzhi), Lentinula edodes (Shiitake), Agaricus blazei Murill, and Inonotus obliquus (Chaga). In a recent Cochrane review, the authors investigated clinical trials that had used G. lucidum extracts against cancer and concluded that this fungus has a promising potential in cancer treatment as an adjuvant by stimulating host immunity, although more research is needed (5). It remains to be seen which compounds are responsible for the immune stimulating effects, but polysaccharides and triterpenoids such as gandoderic acid have been suggested to be important for the bioactivity of this mushroom. L. edodes has attracted interest mainly due to the $\beta$-glucan lentinan, which is used as an adjuvant in cancer therapy in Japan and China. A recent review on lentinan summarized results from several clinical trials involving patients with lung, colorectal or gastric cancer (6). The authors concluded that lentinan was shown to increase efficacy of chemotherapy and radiation treatment, as well as increasing the well-being of patients, and the mechanism of action of lentinan was likely to be through stimulation of the immune system. A. blazei has also attracted interest due to positive immunological effects in clinical trials on patients with inflammatory bowel disease (7). Although the specific compounds responsible for the effects could not be identified, it was speculated that polysaccharides such as $\beta$-glucans and low-molecular weight compounds such as phenolic acids or other antioxidants could be important for the effects. 


\subsection{Inonotus obliquus}

The white-rot fungus Inonotus obliquus (pers.: Fr.) Pilát, popularly known as Chaga, is a fungus traditionally used for medicinal purposes. It belongs to the Hymenochaetaceae family and the Basidiomycota division of fungi. The fungus is characterized by its black, charcoal-like appearance and grows on trees of the Betula and Alder genera in cold climates in the Northern Hemisphere (Figure 1), where it attaches to the stem and slowly degrades lignin and cellulose as it grows (8). Although the fungus is considered a parasite, it might also benefit the host tree by patching up damage on the bark where it initially attaches. After attachment to the tree, the fungus slowly starts producing mycelium, which over time condenses into sterile conks of sclerotia that remain attached on the tree until its death many years or decades later. These sterile conks may vary in size depending on the attachment site and the age of the fungus, with some samples weighing more than $10 \mathrm{~kg}$ and having diameters above $50 \mathrm{~cm}$. I. obliquus differs from most wood-decaying fungi, since it remains in an asexual stage throughout the tree's lifetime and thus cannot spread its spores the way other polypore mushrooms do. Therefore, it is not uncommon to find just a single I. obliquus sclerotium on any given tree, and this might be an important reason why trees are able to live for decades even with an I. obliquus infection. The sclerotia of I. obliquus are sometimes mistakenly called fruiting bodies. Although technically considered a polypore fungus, fruiting bodies of I. obliquus have only been documented a few times, and are usually produced after the tree is dead, although in rare cases they may form on living trees as well (9). During this stage, I. obliquus enters its reproduction cycle once again and may spread its spores to surrounding trees. 


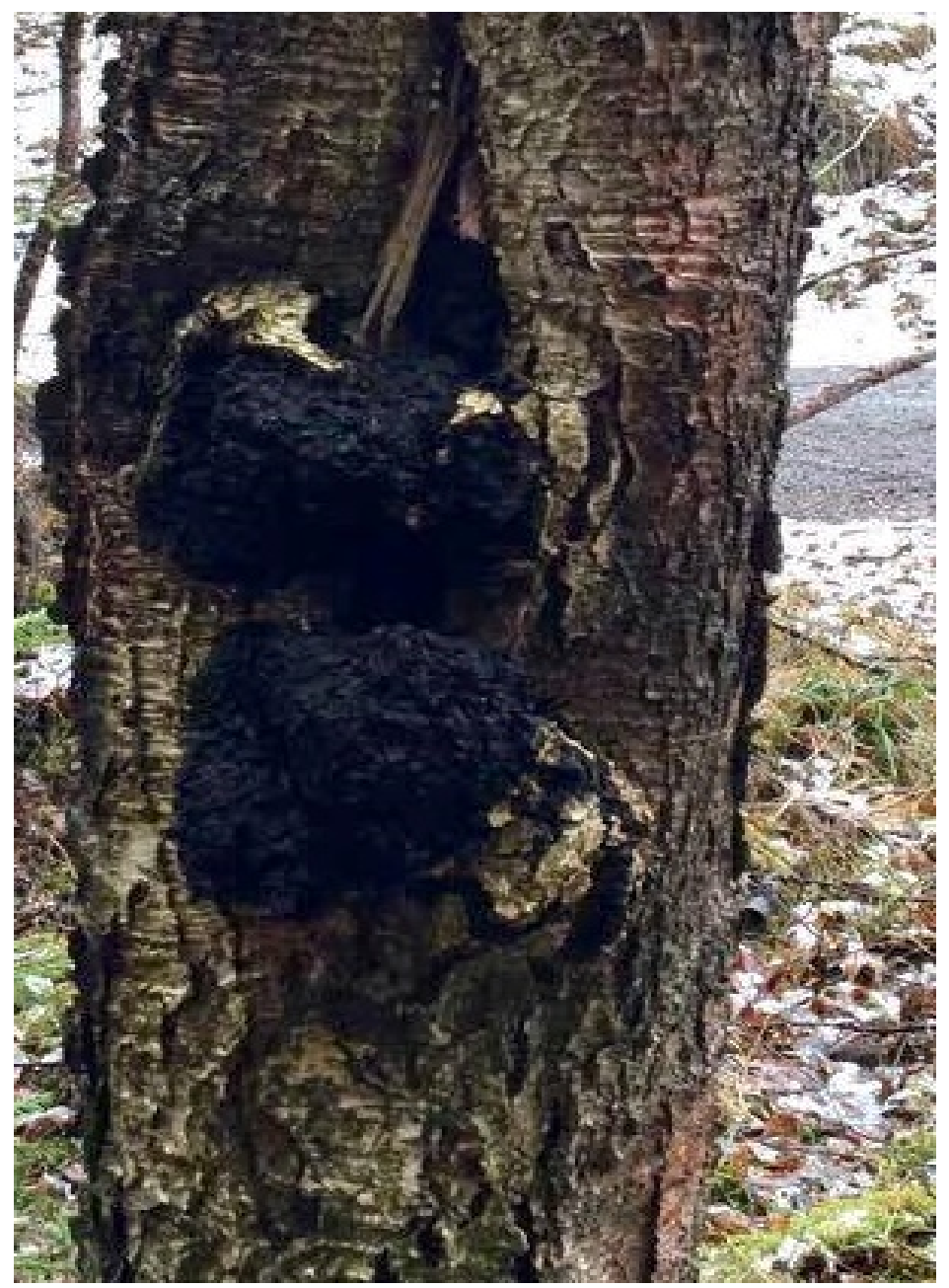

Figure 1. I. obliquus sclerotia growing on a birch tree in the Oslo area (taken during a field trip in April 2017. Photo taken by Cindy Suen).

Usage of I. obliquus in Russia can been dated back to at least the $12^{\text {th }}$ century, where tribal communities like the Khanty people of Siberia used it for medicinal purposes $(10,11)$. The fungus was usually cut into small pieces and boiled in water, before the extract was consumed as a decoction against stomach illnesses or applied to external wounds. Today, a standardized I. obliquus water-ethanol dual extract called Befungin ${ }^{\circledR}$ is available in Russia and is prescribed by doctors for treatment of psoriasis and gastrointestinal disorders (11). I. obliquus also has a long history of use in China, Korea, Japan and Canada, as well as in several Northern and Eastern European countries. For example, the Sámi people of northern Norway are known to use I. obliquus as an alternative to tea and coffee during the winter due to its alleged health promoting properties. The fungus is now registered in the European Union novel food catalogue, where it is listed as a food supplement due to documented use in European countries 
prior to 1997 (12). Popular interest for I. obliquus in western countries can probably be traced back to the book "The Cancer Ward" published in 1966 by the Nobel Prize-winning Russian author Alexander Solzhenitsyn. In this controversial and semi-autobiographical book, Solzhenitsyn mentions consumption of I. obliquus as a way of curing cancer (13). Indeed, the last decades an increasing amount of I. obliquus research has focused on its anti-cancer properties and studies have demonstrated cancer growth inhibition in mice by using I. obliquus extracts $(14,15)$. Polysaccharides with immunomodulating properties have been obtained from I. obliquus, and several authors describe in vivo anti-tumor effects by these polysaccharides, possibly caused by stimulation of the immune system leading to an enhanced immune response against the tumors $(16,17)$. Further, compounds of the triterpenoid class with in vitro cancer cell cytotoxicity have been isolated and characterized, with notable examples being betulinic acid and inotodiol (18). In addition, I. obliquus contains many other compounds with potential medicinal value. One of the components of I. obliquus sclerotia is what many Russians refer to as the "chromogenic complex", which are usually classified as melanin pigments (19). These pigments are produced in massive amounts by the fungus and although poorly characterized they are the main ingredient in water extracts, but their specific biological effects remain to be determined (20). Low molecular weight phenolic compounds have also been characterized, and these often demonstrate strong antioxidative properties in vitro (21).

Despite many promising pre-clinical studies on I. obliquus extracts and metabolites, clinical trials are lacking. Research is also hampered because a significant amount of knowledge is being kept within Russia due to the lack of translated articles. For example, some Russian authors claim that promising clinical trials have been carried out on cancer patients using $I$. obliquus extracts, but these articles are not available in English or even in public databases, and their quality can therefore not be properly assessed by others (11). In the future, such articles should be made available for a broader scientific community, so that research can continue to advance instead of repeating itself. Research on I. obliquus will surely continue for many years to come, hopefully unravelling the many mysteries still surrounding this fungus and provide discoveries that ultimately can benefit the society in one way or the other. 


\section{Biologically active fungal compounds}

As mentioned in section 1, the fungal kingdom is a source of pharmaceutical drugs. In general, fungi contain a wide range of secondary metabolites belonging to different classes of natural products, such as triterpenoids, polyketides, polyphenols, phenolic acids, peptides and alkaloids (22). In addition, many fungi contain polymers like polysaccharides and melanin with immunological properties. One important reason behind the immunological properties is that our immune system has evolved alongside pathogenic fungi, and is able to recognize a broad specter of fungal molecular patterns (23). The classes of fungal compounds that were investigated in this thesis are summarized below.

\subsection{Polysaccharides}

Polysaccharides are complex, long-chained carbohydrates that serve important functions in all living organisms. For humans and other animals, polysaccharides from plants are utilized as an energy source in the form of starch, which are $\alpha$-linked glucans. In plants, fungi and bacteria, polysaccharides serve important structural functions in the cell wall, such as cellulose and pectin in plants, and chitin and $\beta$-glucans in fungi (24). Plant and fungal cell wall polysaccharides can be complex and heterogeneous, and are mainly non-digestible by humans due to the lack of proper enzymes to cleave the glycosidic linkages between the monomers. These polysaccharides can pass untreated through the small intestine as fibers, and some of them eventually end up being degraded and used as a food source (prebiotics) by bacteria in the colon (24). This degradation by the intestinal microbiota leads to production of short-chain fatty acids (SCFAs), compounds that are crucial for gut health and can act as local signaling molecules. In addition, SCFAs are associated with immune regulation in the gut (25) and have also been suggested to induce systemic effects such as improving insulin sensitivity in obese people, although this needs to be verified in randomized clinical trials (26). Another possible fate for polysaccharides entering the gastrointestinal tract is to be detected by immune cells and endothelial cells in the gut, e.g. in Peyer's patches localized throughout the small intestine (27). The innate immune system of animals has co-evolved along with pathogenic fungi, and several immune cells such as neutrophils, macrophages and dendritic cells are capable of recognizing structures from fungal cell walls (23). Figure 2 gives an overview of polymeric structures from pathogenic fungi with immunological effects, demonstrating the complexity of the fungal cell wall and the structural variability between fungal species. 


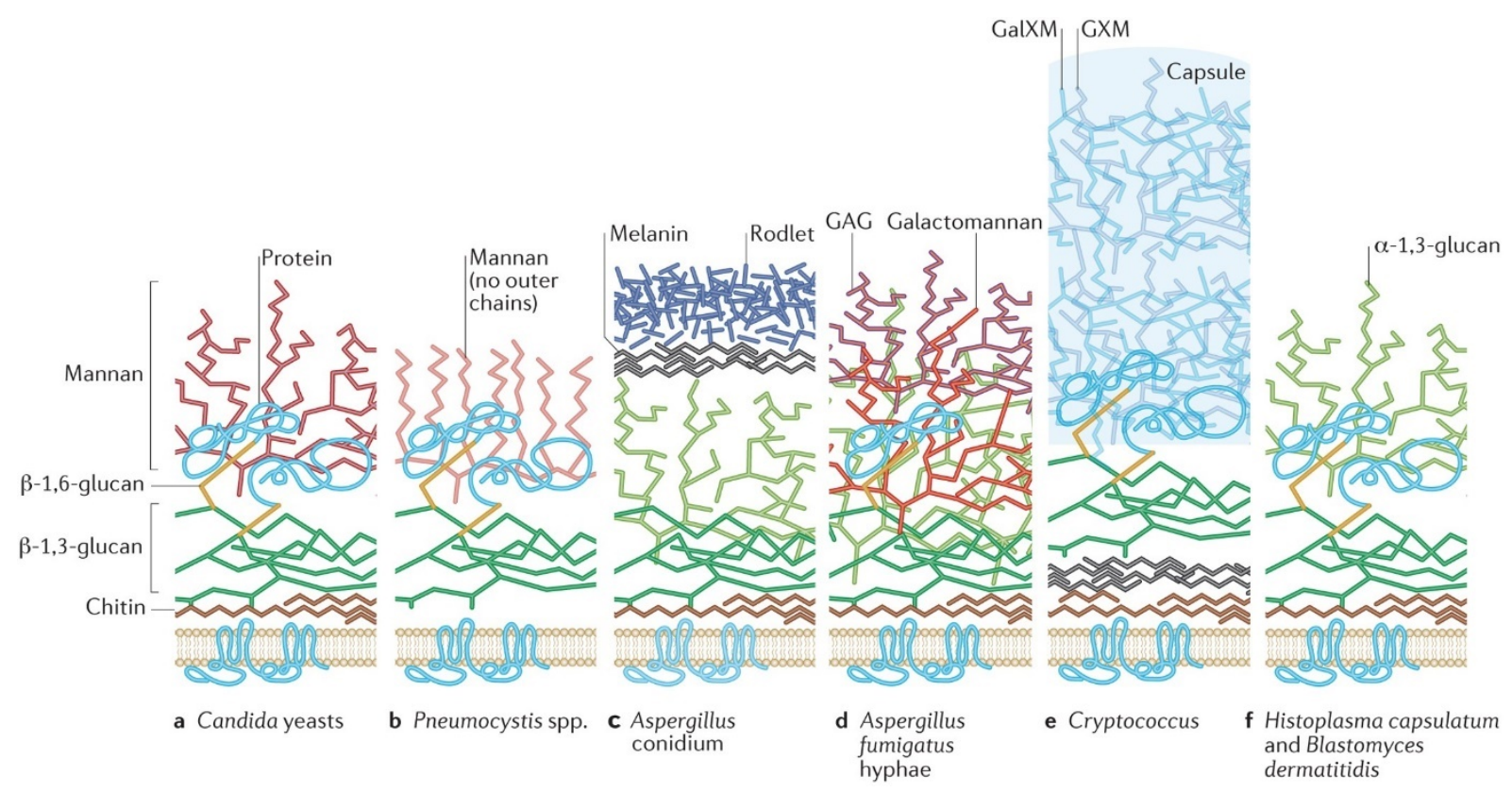

Figure 2. An overview of polymers present in the cell wall of some pathogenic fungi, demonstrating the complexity of the fungal cell wall. Many types of polymers can be present in the fungal cell wall, such as $(1 \rightarrow 3)-\beta$-glucans, $(1 \rightarrow 6)-\beta$-glucans, mannans, chitin, proteins, melanin and heteropolysaccharides such as galactomannans and glucuronoxylomannans (GXM). These polymers can also be cross-linked, further increasing cell wall complexity. From left to right: Candida yeast (a), Pneumocystis spp. (b), Aspergillus conidum (c), Aspergillus fumigaus hyphae (d), Cryptococcus (e) and Histoplasma capsulatum/Blastomyces dermatitidis (f). Adapted from (23) with permission from Springer Nature.

Fungal polysaccharides are a diverse group of polymers, and recognition by immune cells depend on several features of the polymers, including monomeric composition, polymer size, degree of branching, linkage types, anomeric configuration and three-dimensional confirmation (28). An important class of fungal polysaccharides are the $\beta$-glucans. As the name implies, these polymers consist of glucose (Glc) monomers that are linked by $\beta$-glycosidic linkages. Fungal $\beta$-glucans are usually $(1 \rightarrow 3) /(1 \rightarrow 6)$-linked polymers (Figure 3 ), although variations in the basic structure are commonly found (28). 


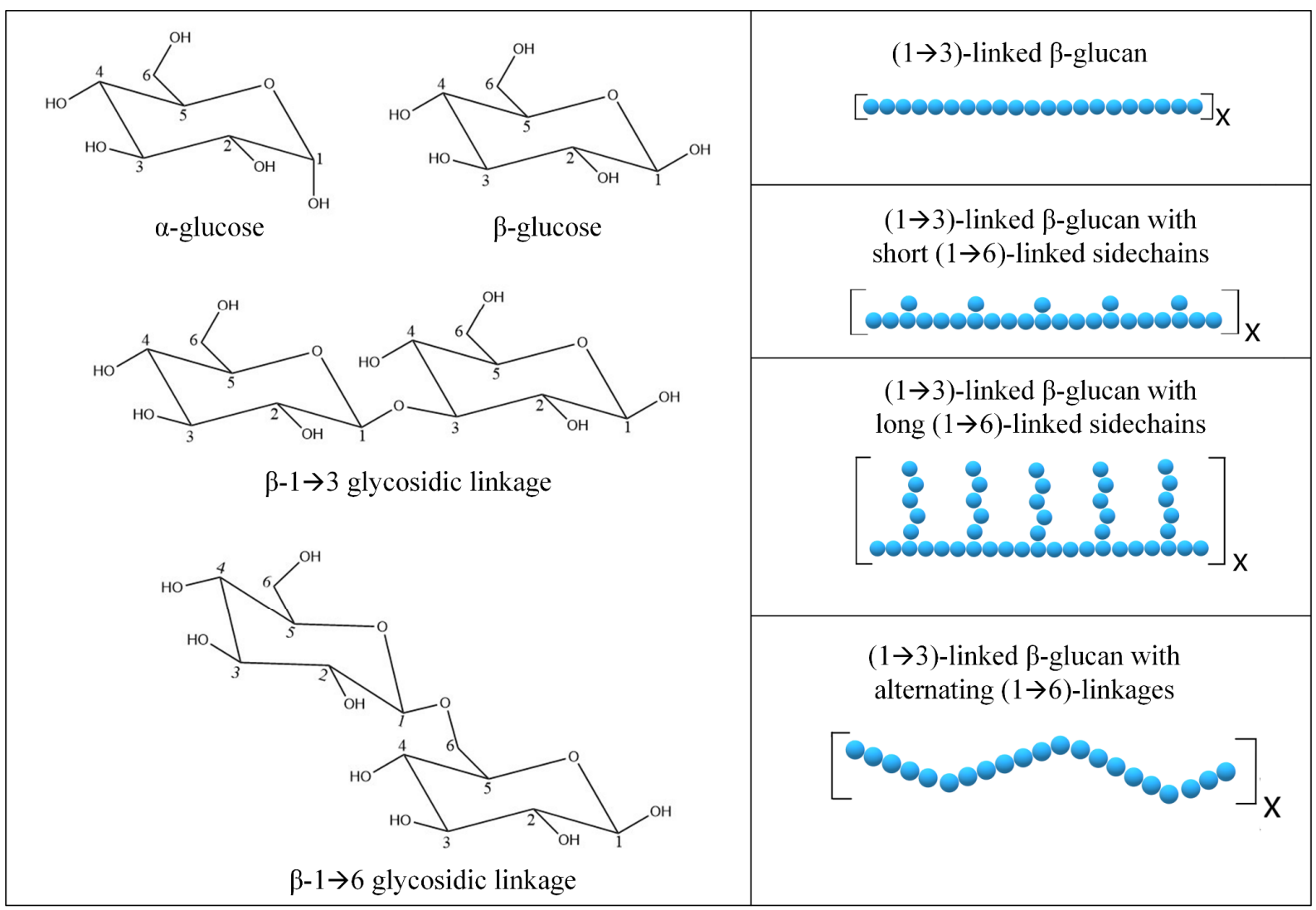

Figure 3. Chemistry of glucose and $\beta$-glucans. Left: $\alpha$ - and $\beta$ - anomeric configuration of Glc, and $1 \rightarrow 3$-linked and $1 \rightarrow 6$-linked Glc monomers. Numbers designate the carbon atoms starting with the anomeric carbon (C1). Right: Variations in the basic $\beta$-glucan structure depending on the relationship and ratio between $(1 \rightarrow 3)$-linkages and $(1 \rightarrow 6)$-linkages.

Fungal $\beta$-glucans with $(1 \rightarrow 3) /(1 \rightarrow 6)$ linkages are known to possess immunomodulating activity (29). On the other hand, $\beta$-glucans from plants such as oat and barley are $(1 \rightarrow 4) /(1 \rightarrow 3)$-linked polymers (30) that are mainly known for their cholesterol-lowering effects through increasing viscosity in the gut, and these polymers are not considered immunologically active (31). The linkage configuration denotes how the Glc monomers are linked together. For example, a $(1 \rightarrow 3)$ linkage means that carbon atom $1(\mathrm{C} 1)$ on a given Glc molecule is attached through a glycosidic linkage to carbon atom 3 (C3) on the next Glc molecule, which will affect the three dimensional shape of the polymer.

Alkaline-extracted fungal $\beta$-glucans have high molecular weights, tend to be insoluble (particulate) in water, and usually have one long chain of $(1 \rightarrow 3)$-linked monomers and shorter $(1 \rightarrow 6)$-linked branches. $(1 \rightarrow 6)$-Linkages interspersed within the main chain can also exist, such 
as for the $\beta$-glucan lentinan from L. edodes (32). The water solubility of the polymers is highly dependent on the molecular weight. For example, it was reported that lentinan was insoluble in its full size of $1,000 \mathrm{kDa}$, but when using partial hydrolysis to reduce the molecular weight to $16 \mathrm{kDa}$, the polymer became soluble in water (32). Fungal $\beta$-glucans are reported to form single or triple helices or random coils in aqueous solutions, but the conformation depends on many factors, such as the linkage type, anomeric configuration, degree of branching, type of solvent and $\mathrm{pH}(27)$.

In addition to $\beta$-glucans, several other types of polysaccharides are commonly found in fungi. Chitin, a polysaccharide consisting of $(1 \rightarrow 4)$-linked $\beta-N$-acetylglucosamine units, is one of the most common fungal cell wall polysaccharides, and the presence of chitin is considered an important defining feature when characterizing fungi from soil samples (2). Another common cell-wall polysaccharide in fungi is $\alpha$-mannan, which is often bound to proteins, such as is the case in Saccharomyces cerevisiae (baker's yeast) (33). Interestingly, it has been suggested that mannoproteins, which make up the outer part of the cell wall, are used as an immune evasion strategy by pathogenic fungi, because they are able to hide the more immunologically active $\beta$ glucans, thus rendering them less exposed to the exterior environment (34).

Apart from homogenous polysaccharides, there are numerous reports on complex, heterogeneous polysaccharides from fungi, and these may consist of many different monomers, types of linkages and both $\alpha$ - and $\beta$-configurations (23). For example, several research groups, including our own group, have isolated and characterized water-soluble heteropolysaccharides from I. obliquus $(16,20,35)$. These polysaccharides are usually water-soluble and smaller than the pure $\beta$-glucans, giving them other properties that might be worthwhile investigating. For example, the heterogeneity of these polysaccharides might affect their immunomodulating effects, as some of them might contain $\beta$-glucan motifs that are recognized by $\beta$-glucan detecting immune receptors, in addition to other structural motifs that might produce different effects by binding to other types of receptors.

\subsection{Triterpenoids}

Terpenes are secondary metabolites with ubiquitous distribution in nature, which are often used as protective agents by plants and fungi against predators and microbial attacks and to communicate with surrounding organisms (36). The terpenes are usually divided into different classes based on the number of isoprene units in their carbon skeleton (37), with the most 
common types being monoterpenes $(\mathrm{C} 10)$, sequiterpenes $(\mathrm{C} 15)$, diterpenes $(\mathrm{C} 20)$, triterpenes (C30) and tetraterpenes (C40). Fungal triterpenoids, which are oxygenated triterpenes, have attracted attention due to their interesting biological activities (38). Triterpenoids usually have a high carbon to oxygen ratio, making them poorly soluble in aqueous solutions, therefore organic solvents such as ethanol or dichloromethane (DCM) are used to extract them from the fungal material.

Many fungal triterpenoids are reported to have cytotoxic effects on human cancer cell lines, such as ganoderic acids isolated from G. lucidum (39). Dozens of such compounds have also been isolated from I. obliquus throughout the years $(40,41)$. Some of them, in particular inotodiol and betulinic acid (Figure 4), have been extensively investigated because of their cytotoxic potential against many types of cancer cells.

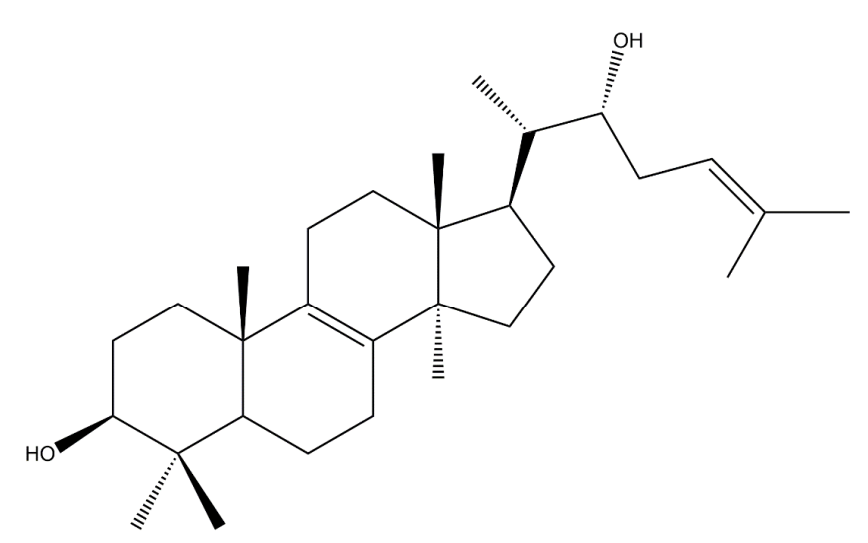

Inotodiol

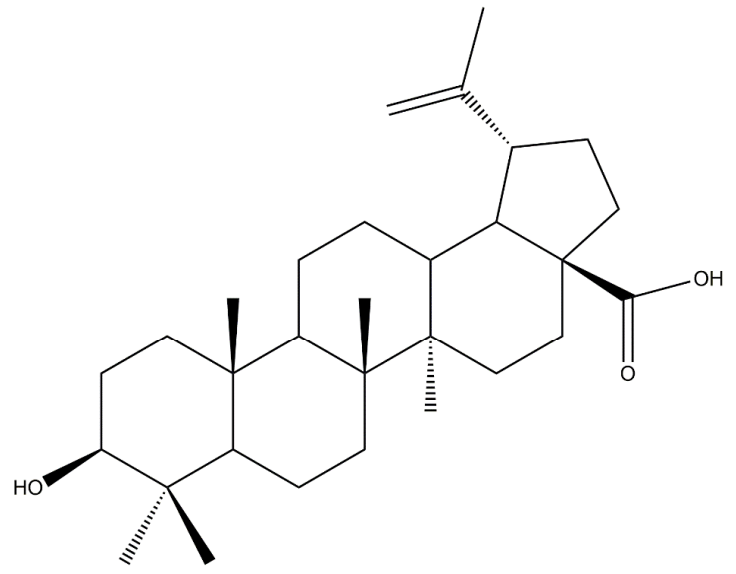

Betulinic acid

Figure 4. Inotodiol and betulinic acid, two important triterpenoids found in I. obliquus.

Inotodiol has been shown to induce apoptosis though a p53-dependent pathway in HeLa cervical cells (42), and to induce apoptosis by caspase-3 activation in the leukemia cell line p388 (43). While inotodiol is a compound that seems to be exclusive to the Inonotus genus (SciFinder search, 03/06-2020), betulinic acid has been isolated from a variety of different plant species. The origin of its name comes from the chemically related triterpenoid betulin, which is found in abundance in birch bark (44). Betulinic acid is known to induce apoptosis in melanoma cells (45). Although previously believed that it was selective against melanoma, it has later become evident that betulinic acid has cytotoxic and cytostatic effects toward many types of cancer cells (46). Betulinic acid has been extensively researched, and there have been 
efforts to develop synthetic derivatives of this compound in order to increase its cytotoxicity and bioavailability (47). Apart from its cytotoxic effects, inotodiol was recently shown to prevent food-induced allergy in a mouse study by selectively inhibiting mast cells (48), and the betulinic acid-derived drug Bevirimat has been shown to have anti-HIV activity in clinical trials (49).

\subsection{Melanin}

Melanin is a pigment widely distributed in nature, and is found in all kingdoms of life. The function of melanin is often to protect the organism against environmental stressors such as UV light (50). Although the distribution of melanin is widespread, there are still many unknown aspects regarding its functions and detailed chemical structure. Animal melanin, such as found in human skin and hair, are usually termed eumelanin or pheomelanin. While eumelanin is black or brown in appearance and protects against UV radiation, pheomelanin has a reddish color and can lead to increased susceptibility against UV light (51). Both eumelanin and pheomelanin synthesis begins with oxidation of L-tyrosine to dopaquinone and subsequent polymerization of similar structures, but the pathways differ due to incorporation of L-cysteine into the pheomelanin pathway at the dopaquinone step (51).

Fungal melanin pigments are less characterized than animal melanin, and structural composition seems to vary from species to species and may be dependent on environmental factors as well (50). There are two main pathways associated with fungal melanin synthesis (Figure 5). In one of them, the monomeric units are tyrosine-derived molecules similar to the ones found in animal eumelanin. In the other, sometimes called the allomelanin or polyketide melanin pathway, the pigment is polymerized from 1,8-dihydroxynaphthalene $(1,8$-DHN) or similar units (52). 
<smiles>Oc1cc2cc[nH]c2cc1O</smiles>

5,6-dihydroxyindole (DHI)<smiles>O=C(O)c1cc2cc(O)c(O)cc2[nH]1</smiles>
$\mathrm{OOH}$

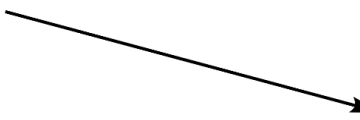

Polymerization
Eumelanin (L-tyrosinederived melanin)

5,6-dihydroxyindole-

2-carboxylic acid (DHICA)<smiles>Oc1cccc2cccc(O)c12</smiles>

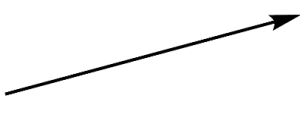


characterized, and it has been suggested that the chemical composition may vary depending on if the fungus is growing in the wild or in culture (56). Because I. obliquus is exclusively found in cold climates in the Northern Hemisphere, it seems unlikely that the fungus produces melanin as a way of protecting itself from UV radiation, implying that it is used for other purposes. Indeed, it has been suggested that melanin pigments can serve a wide variety of biological functions, and apart from being a virulence factor, melanin might be involved in thermoregulation and provide mechanical rigidity, both of which seem plausible functions for melanin in I. obliquus (50).

\section{The immune system}

Our immune system constantly protects us from external and internal threats, and consists of a large number of different cell types as well as secreted proteins such as antibodies (57). This complex system is usually divided into two main parts depending on the specificity of the response: the innate and the adaptive immune system (Figure 6). The innate immune system is part of our first line of defense. In addition to physical barriers such the skin and mucus layers, the innate immune system is characterized by its ability to recognize pathogen associated- and damage-associated molecular patterns (PAMPs and DAMPs) from e.g. invading pathogens and apoptotic debris. Upon recognition of such structural motifs, a non-specific immune response against the threat is rapidly generated, which can be through different mechanisms such as secretion of toxic molecules or engulfment of the foreign material by phagocytic cells (57). The innate immune system consists of several types of effector cells, the most important being macrophages, dendritic cells, natural killer (NK) cells, neutrophils, basophils and eosinophils. In addition, the complement system, consisting of a series of secreted proteins, is usually considered as a part of the innate immune response (58). Upon activation, the innate immune system is able to communicate the nature of the threat with cells from the adaptive immune system (T- and B-cells), and this will ultimately lead to the generation of a specific (adaptive) immune response. 


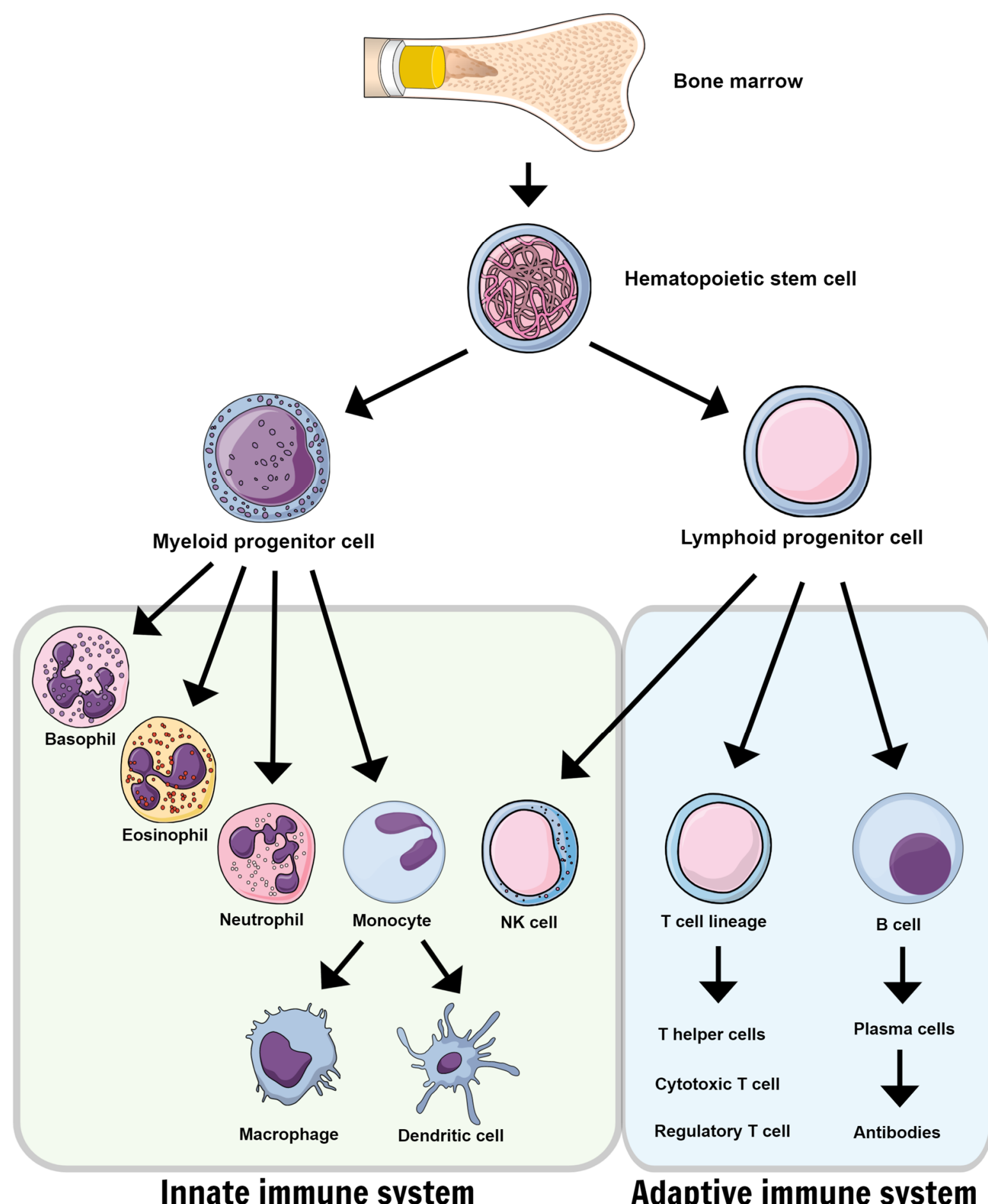

Innate immune system

Figure 6. Cell lineages of the innate and adaptive immune system. Virtually all immune cells are derived from hematopoietic stem cells in the bone marrow, which are differentiated into two separate linages, myeloid and lymphoid progenitor cells, giving rise to innate and adaptive immune cells, respectively. An exception to this rule is the natural killer (NK) cells, which are considered a part of the innate immune system but are differentiated from lymphoid progenitor cells. (Figure created using Mind the Graph) 
Fungal compounds may interact with the immune system in many ways. The polysaccharide lentinan from shiitake mushrooms is used as an adjuvant in cancer therapy in several countries due to its immunomodulating properties (59), the cyclic peptide cyclosporine isolated from soil fungi is used as an immunosuppressant in organ transplantation (60), and dozens of secondary metabolites from marine fungi show promise as anti-inflammatory agents in pre-clinical research (61). These examples emphasize the importance of discovering new types of fungal metabolites, which might prove beneficial in a world where the number of people with autoimmune diseases, chronic inflammation and cancer are rapidly increasing.

\subsection{The complement system}

Complement is an integral part of the innate immune system and, among other things, functions as a bridge between innate and adaptive immunity (62). As the name implies, the complement system is regarded as a supplement (or complement) to the other parts of the immune system. Although this is true to some extent, complement was actually one of the earliest defense mechanisms we evolved, and in addition to being a crucial part of our immune system, complement is important for a variety of other homeostatic processes not related to the immune response (58). The main function of complement is to recognize PAMPs and DAMPs. The complement system consists of a large number of circulating proteins, which upon recognition of PAMPs (or DAMPs) may trigger an activation cascade leading to the recruitment of effector immune cells and the destruction of e.g. invading pathogens (58). Although a simplification, it is generally accepted that the complement activation cascade is triggered through three different pathways (Figure 7) (62). 


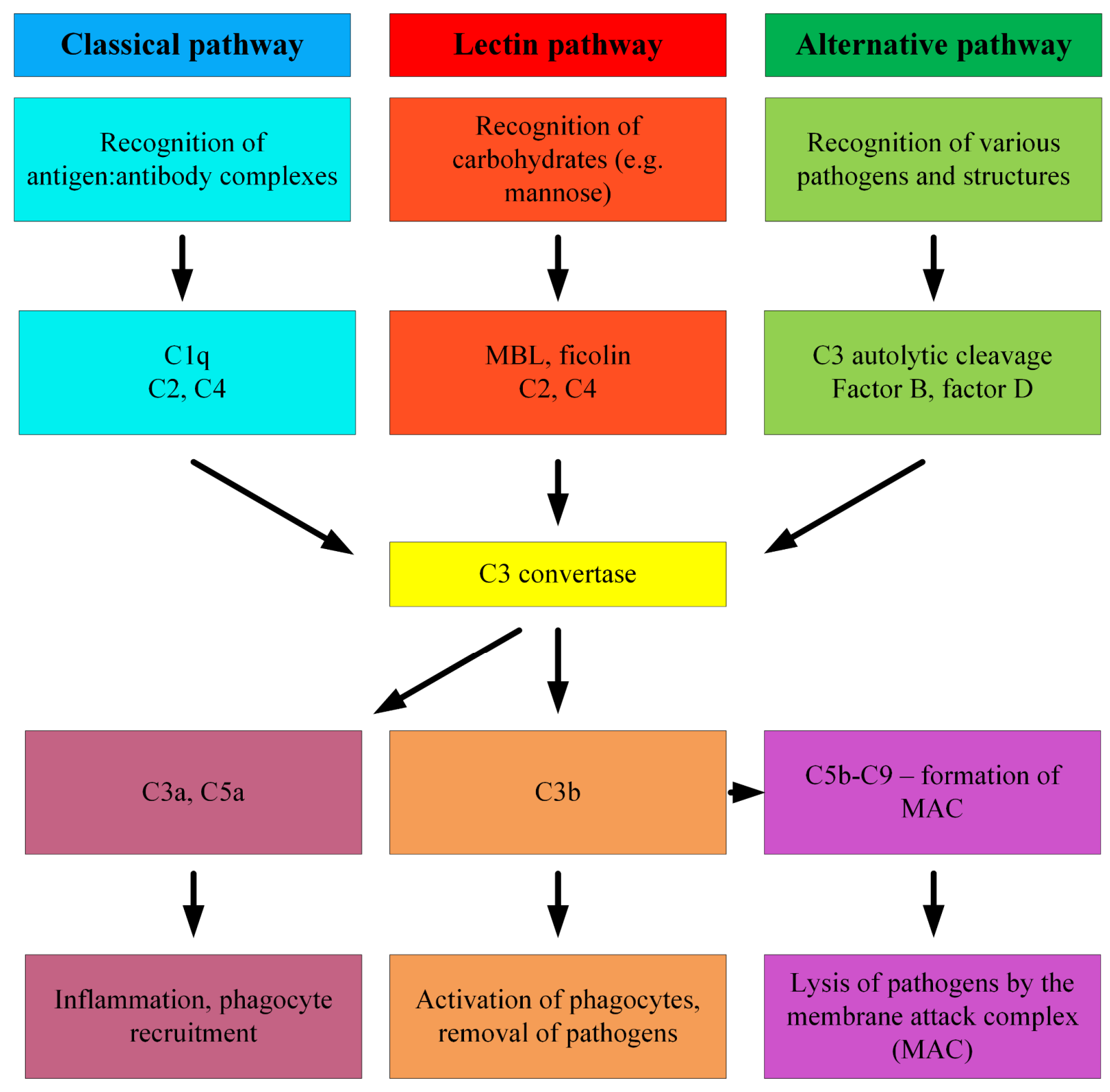

Figure 7. The complement cascade is triggered through three distinct pathways, based on which danger signals are present. Activation of complement leads to similar outcomes regardless of the pathway, ending up with recruitment of immune cells and clearance of the threat, which can be for example invading pathogens or apoptotic debris.

The classical pathway is known as the antibody-dependent pathway, and is initiated when a complement protein called $\mathrm{C} 1 \mathrm{q}$ binds to the $\mathrm{Fc}$ region of antibodies bound to antigens. The lectin pathway recognizes carbohydrate structures on microbes such as bacteria and fungi, and involves some of the same proteins as the classical pathway, such as $\mathrm{C} 2$ and $\mathrm{C} 4$. The alternative pathway can be triggered by many types of structural motifs. This pathway involves spontaneous cleavage of $\mathrm{C} 3$ into $\mathrm{C} 3 \mathrm{a}$ and $\mathrm{C} 3 \mathrm{~b}$, meaning that this pathway is constitutively turned on (at low levels) and acts as a constant surveillance system against invading threats. 
Activation of any of the three pathways leads to several outcomes. First, some complement proteins (e.g. $\mathrm{C} 3 \mathrm{a}$ and $\mathrm{C} 5 \mathrm{a}$ ) function as chemoattractants to recruit immune cells such as phagocytes to the activation site. Further, activated complement proteins (e.g. C3b) can bind to complement receptors on macrophages and other phagocytic cells. This leads to an activation of the phagocytes and subsequent uptake and killing of the microbes, which may ultimately lead to presentation of the antigens to $\mathrm{CD} 4^{+} \mathrm{T}$-cells and thus activation of adaptive immunity. Another mechanism of action by the complement cascade is the assembly of complement proteins (C5b-C9) into a membrane attack complex (MAC), which is able to destroy the cell membrane of the invading microbes (58). Thus, activation of the complement cascade can lead to a strong immune response against both external and internal threats through multiple mechanisms.

Dysregulation of complement has been linked to the development of a wide range of diseases and pathological conditions such as cancer, and compounds that can modulate the activity of complement could therefore represent new types of pharmacotherapies (63). The role of the complement system in cancer development is complex, and it is thought that complement can act both tumor suppressive and tumor promoting (63). For example, it has been suggested that strong activation of complement in the tumor microenvironment could lead to generation of MAC, which eventually could destroy cancer cells (64). Some tumors, on the other hand, are known to produce complement-suppressing factors and can use different evasion strategies to escape complement-induced immune responses e.g. by downregulating membrane-bound complement receptors (64). Therefore, antibodies or other compounds that can target and block these suppressive responses could be attractive treatment options in cancer immunotherapy. Indeed, due to the increasing knowledge of the underlying mechanisms of complement activation, it seems evident that most other immune responses in one way or the other are linked to complement activation, and therefore this system will need to be taken into consideration when new drugs against immune related diseases are developed (58).

\subsection{Macrophages and dendritic cells}

Macrophages, dendritic cells and neutrophils are phagocytic cells, which are cells able to "eat" and engulf microbes, damaged cells or other components from their local environment. After uptake, phagocytes usually kill or degrade the material they have taken up by producing large amounts of reactive oxygen- and nitrogen species in a process known as respiratory burst (65). 
In addition, macrophages and dendritic cells in particular are known as antigen presenting cells, and are capable of presenting the degraded material as antigens to T-cells such as $\mathrm{CD} 4^{+} \mathrm{T}$ helper cells (57). Although a simplification, this antigen presentation will usually initiate an adaptive immune response, leading to a highly specific response toward the threat. Additionally, the phagocytes can themselves become activated by the antigen uptake or through recognition of PAMPs by PRRs on their cell membrane, and start to secrete cytokines and other pro-inflammatory signals into the local environment that might kill microbes or cancer cells and recruit other effector cells to the damaged site (66). An overview of some important functions of macrophages is shown in Figure 8.

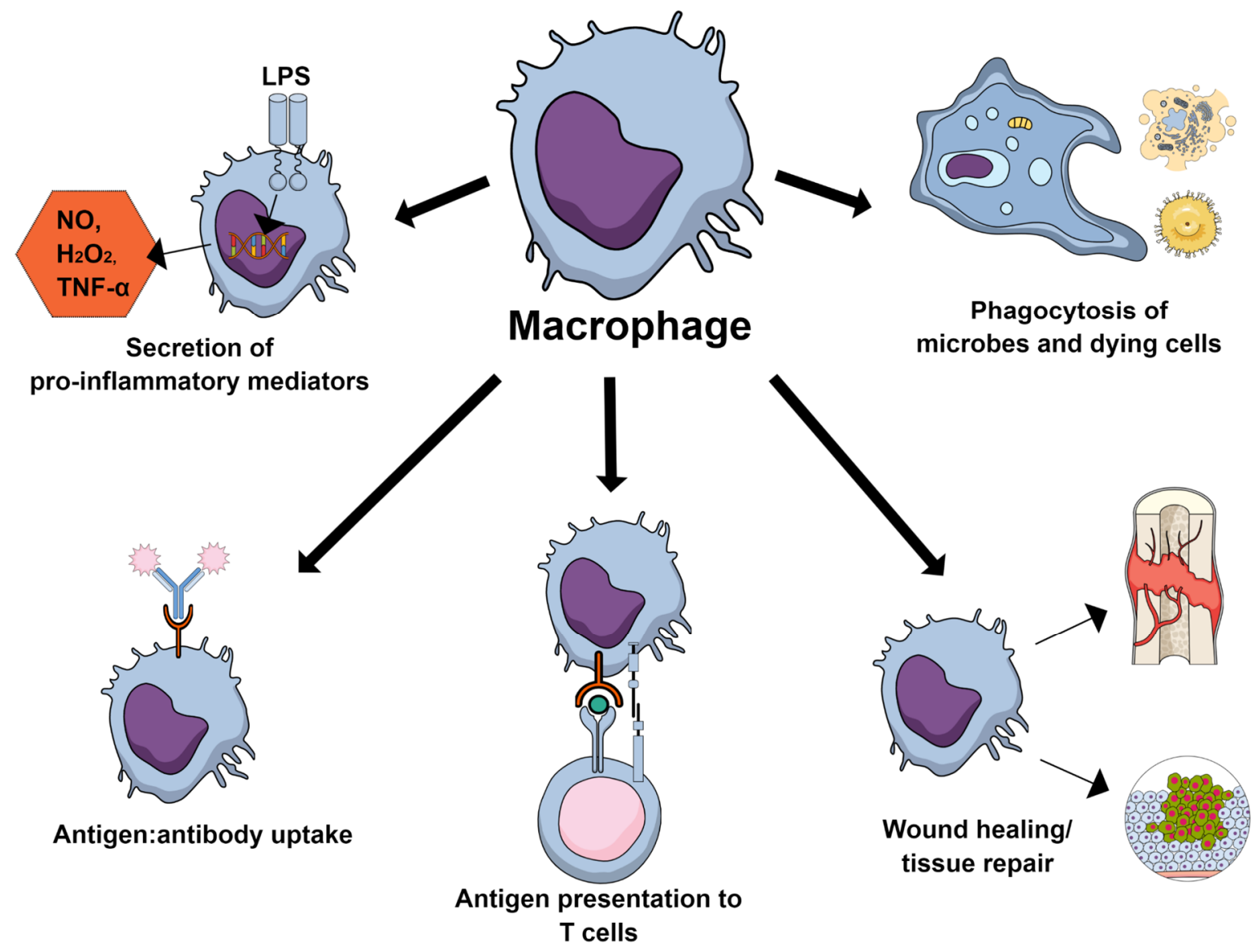

Figure 8. Macrophages have a wide variety of functions, which are usually dependent on the local environment.

Top left: Macrophages can aquire a proinflammatory phenotype upon stimulation by DAMPs and PAMPs (e.g. LPS). Bottom left: Macrophages can interalize antigen:antibody complexes. Bottom: Macrophages can present antigens to T cells, leading to an adaptive immune response. Bottom right: Macrophages are able to repair wounds by providing growth factors and other stimuli to damaged tissues. Top right: Macrophages can phagocytose microbes and apoptotic/necrotic cells. (Figure created using Mind the Graph) 
Although the phagocytic cell types have many similarities, they are quite different in many aspects. For example, neutrophils are short-lived (hours), blood-circulating cells that mainly act on their own by killing invading microbes. Macrophages and dendritic cells, on the other hand, are longer-lived (days/weeks) cells that are distributed in tissues (both) and lymph nodes (dendritic cells) throughout the body. Both these cell types are highly dynamic, and their phenotype and specific functions depend greatly on their local environment and which signals they receive from surrounding cells. Thus, these cells have the capacity to be modulated or polarized into certain phenotypes, and this ability gives them the potential to be utilized for development of drugs for novel therapies against cancer and autoimmune diseases. This is also evident from the recent interest for macrophage-directed cancer immunotherapies by the pharmaceutical industry (67). In addition, macrophages and dendritic cells are able to recognize many types of structures from fungal cell walls, and are attractive targets when it comes to investigating new types of immunomodulating compounds from fungi $(23,68)$.

\subsubsection{Tumor-associated macrophages}

In most solid tumors, the tumor microenvironment contains a huge number of immune cells, and knowledge about this immune cell composition can be used as a predictive tool for disease outcome and for treatment optimization (69). Due to their dynamic and ever-changing nature, macrophages present in the tumor microenvironment may have either anti- or pro-tumorigenic phenotypes, $(66,70,71)$. For example, tumor-associated macrophages (TAMs) can act protumorigenic by dampening the general immune response against the tumor and by providing growth factors and other signals to the tumor cells $(72,73)$. Macrophages are thought to gain their phenotypes through interaction with signal molecules from surrounding cells. Interferon gamma (IFN- $\gamma$ ), a cytokine produced by type $1 \mathrm{~T}$ helper $\left(\mathrm{T}_{\mathrm{h}} 1\right)$ cells, is thought to drive macrophages toward a pro-inflammatory phenotype. On the opposite side of this axis, interleukin-4 (IL-4), a cytokine produced by type $2 \mathrm{~T}$ helper $\left(\mathrm{T}_{\mathrm{h}} 2\right)$ cells, are thought to induce an anti-inflammatory phenotype in the macrophages, giving them a "repair-mode" phenotype. This macrophage polarization scheme induced by $T_{h} 1$ and $T_{h} 2$ cells eventually lead to the designation "M1" and "M2" macrophages, respectively, depending on which signals they received from $\mathrm{T}$ cells $(73,74)$. However, it is now clear that this is an over-simplification of the truth, and a wide range of macrophage phenotypes are known to exist, questioning the M1/M2 nomenclature (75). Although macrophage activation is a subject of debate, it seems clear that stimulation with toll-like receptor (TLR) ligands such as lipopolysaccharide (LPS) or 
$\mathrm{Pam}_{3} \mathrm{CSK}_{4}$ plus IFN- $\gamma$ induces a pro-inflammatory phenotype and upregulation of proinflammatory mediators such as nitric oxide (NO) and cytokines like tumor necrosis factor alpha (TNF- $\alpha$ ), which in turn might cause anti-tumorigenic effects (76). An activation of TAMs could induce an anti-tumor response by several distinct mechanisms, such as local tissue damage by NO and other reactive compounds, increased uptake of tumor neoantigens by the macrophages with subsequent presentation of the antigens to $\mathrm{CD} 4^{+} \mathrm{T}$-cells, as well as activation of NK cells and cytotoxic $\mathrm{CD}^{+} \mathrm{T}$-cells $(66,73,77)$. Although the full potential of macrophages in cancer therapy remains to be unleashed, several authors emphasize the importance these cells could have in cancer immunotherapy $(66,73)$. For example, it could be that future therapies need to induce activation of antigen-presenting cells such as dendritic cells or macrophages in order for an anti-tumor T-cell response to function efficiently (78). How this should be carried out in detail remains to be seen, but it seems clear that antigen-presenting, phagocytic cells will be important players in cancer treatment regimes in the future.

\subsection{Pattern recognition receptors}

One of the main ways that macrophages are activated into a pro-inflammatory phenotype is through the detection of PAMPs from invading pathogens. This usually happens via pattern recognition receptors (PRRs) present in the cell membrane, in endosomal membranes and in the cytosol (79). As mentioned earlier, these receptors (or sensors) have evolved to recognize general structural motifs from invading microorganisms, damaged tissues and apoptotic debris, and can detect a variety of different classes of molecules, including proteins, carbohydrates, lipids and nucleic acids. The PRRs can be divided into classes or families based on structural similarities between the receptors. One of the main classes, the Toll-like receptors (TLRs), consists of at least ten different receptors in humans, and this class is considered crucial for sensing bacterial and viral structures. In addition to the TLRs, other important classes of PRRs include the NOD-like receptors (NLRs), the RIG-1-like receptors (RLRs) and C-type-lectin receptors (CLRs) (79). As shown in Figure 9, the cellular distribution of the PRRs depends on the receptor family. In addition to the mentioned PRR families, macrophages can indirectly detect PAMPs upon activation of complement through complement receptors such as CR3 present in the cell membrane (58). 


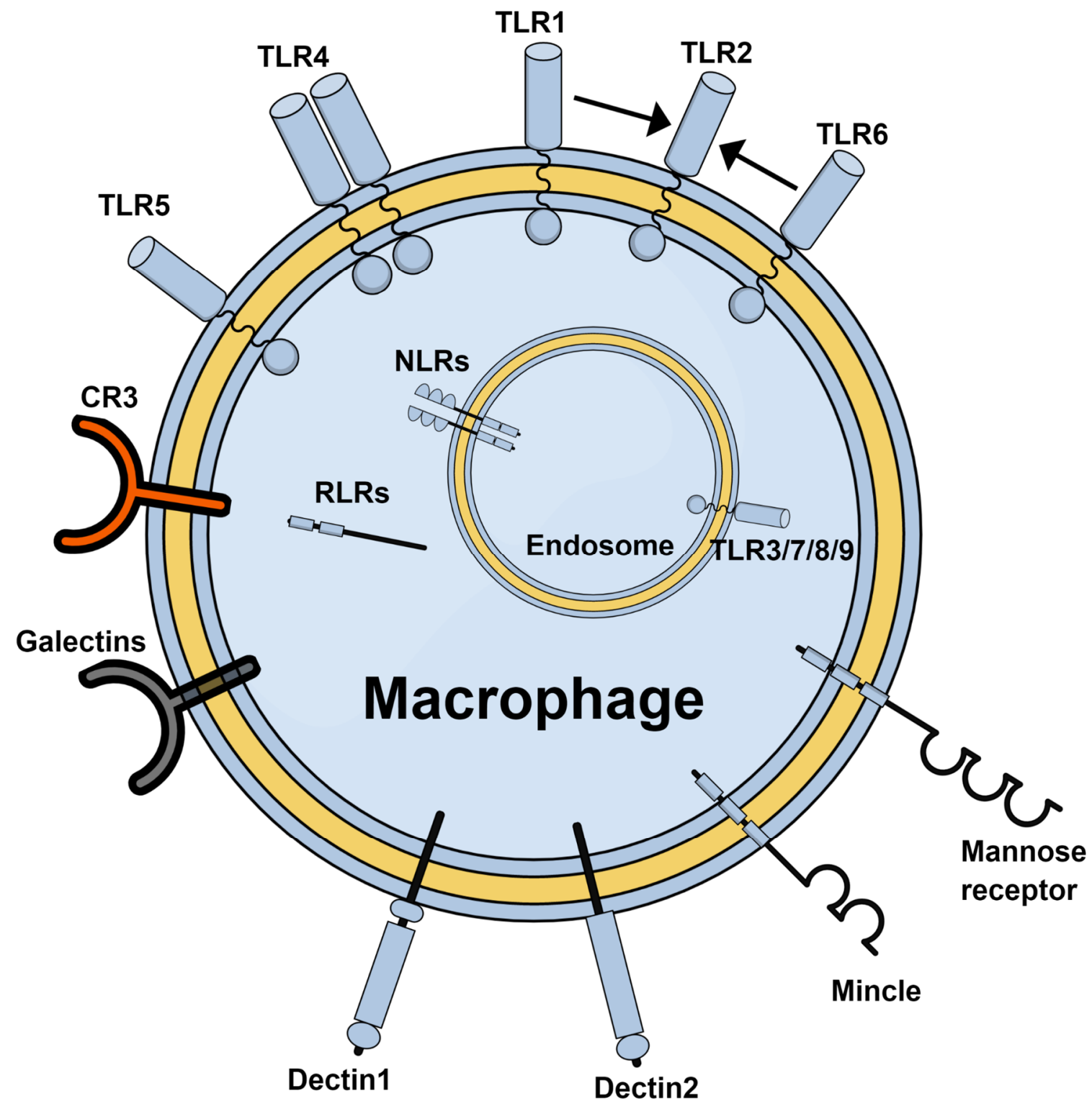

Figure 9. Macrophages express many different types of pattern recognition receptors (PRRs). Toll-like receptors (TLRs) are located either in the cell membrane (TLR1, 2, 4, 5 and 6) or in endosomal membranes (TLR3, 7, 8, 9). The C-type lectin receptors (CLRs) Dectin-1, Dectin-2, Mincle and the mannose receptor are located in the cell membrane. RIG1-like receptors (RLRs) are located in the cytosol, while NOD-like receptors (NLRs) can be located in both the cytosol and in endosomal membranes. In addition, other PRRs such as Galectin-3 and complement receptor 3 (CR3) are important receptors for detecting microbial structures. (Figure created using Mind the Graph) 


\subsubsection{Toll-like receptors}

The TLRs are membrane-spanning receptors present in macrophages and dendritic cells, and this family of receptors was the first of the PRR families to be described (80). The TLRs are able to sense microbial structures and induce a subsequent pro-inflammatory response through a signaling cascade initiated by their intracellular domain. Most TLRs except TLR3 initiate this signaling cascade through the adaptor protein MyD88, which ultimately activates the transcription factors NF-KB and AP-1 (80). TLR3 is activated through another adaptor protein called TRIF, activating the transcription factor IRF-1. TLR4, the receptor that detects LPS, is able to signal through both MyD88 and TRIF, leading to an activation of both pathways (81). Several of the TLRs are dependent on co-receptors to function properly. For example, TLR4 needs to associate with the membrane-bound proteins CD14 and MD-2. In addition, to induce potent TLR4 responses LPS needs to bind the soluble factor LPS-binding protein (LBP) in order to bind the TLR4-MD2-CD14 complex effectively (81). TLR2, on the other hand, does not function as a homodimer, and requires dimerization with either TLR1 or TLR6 to induce intracellular responses (82). The TLR2 $+1 / 6$ heterodimer is able to recognize a broad range of molecular patterns, such as lipoproteins. Interestingly, fungal pathogens and polysaccharides seem to be able to bind both TLR2 and TLR4, although direct mechanisms for this interaction have not yet been described (83).

\subsubsection{C-type lectin receptors}

In addition to the TLRs, the CLRs are present in abundance on the cell membrane of phagocytic cells. CLRs are usually associated with anti-fungal immunity, although it is now evident that they are involved in a variety of disease states such as cancer, allergy and autoimmunity, possibly due to its induction of adaptive immune responses through $\mathrm{T}_{\mathrm{h}} 17$ cells $(84,85)$. As the name C-type lectin implies, these receptors are calcium dependent receptors able to bind carbohydrates, although other types of structures such as DHN-type melanin can also be recognized by some CLRs (54). Similar to the TLRs, the CLRs can be divided into two broad groups based on their intracellular signaling pathways: spleen tyrosine kinase (Syk) dependent or -independent receptors (85). The $\beta$-glucan receptor Dectin-1 belongs to the Syk-dependent group of CLRs, and this receptor is considered one of the most important anti-fungal receptors, due to its ability to selectively detect $(1 \rightarrow 3)$ - and $(1 \rightarrow 6)$-linked $\beta$-glucans $(86)$. $\beta$-Glucans are one of the most common structural motifs in the fungal cell wall, and activation of Dectin-1 by 
such structures can lead to a strong immunological response. One of the hallmarks of a strong activation of Dectin-1 is the formation of a "phagocytic synapse" that will lead to engulfment of the $\beta$-glucan particle (87). This mechanism of action makes some logical sense, because fungal cells are very large, and it is important that they are removed from circulation and phagocytosed before they are able to cause damage to the surrounding tissue. Interestingly, particulate $\beta$-glucans trigger a strong Dectin-1 activation, while smaller, water-soluble $\beta$ glucans do not (87). Although speculative, it could be that smaller and soluble $\beta$-glucans are a sign of degraded fungal cell walls, and therefore do not pose as big a threat as particulate $\beta$ glucans that would still be part of the intact cell wall of a living fungal cell. In addition to Dectin-1, Dectin-2, Mincle, DC-SIGN and CD206 (known as the mannose receptor) are other CLRs important for anti-fungal immunity. Further, apart from signaling on their own, CLRs such as Dectin-1 are able to form receptor complexes with TLRs, in particular with TLR2 and TLR4, giving additional complexity to innate immune responses against fungal threats $(88,89)$.

\section{Immunomodulating polysaccharides from fungi in cancer therapy}

Fungal polysaccharides have been investigated for their anti-cancer potential, for example due to their ability to induce immune responses through activation of macrophages (23). A large number of in vitro studies have been carried out demonstrating that fungal polysaccharides are able to increase e.g. NO, TNF- $\alpha$ and interleukin (IL) production in macrophages, which has been suggested to increase their anti-tumor potential (90). In vivo experiments have demonstrated that polysaccharides from fungi such as I. obliquus were able to reduce tumorburden and increase survival in tumor-bearing mice $(16,17)$. Polysaccharides could also give synergistic anti-tumor effects in mice when combined with chemotherapeutic drugs such as 5fluorouracil by improving immune function $(90,91)$. Further, a few polysaccharides, including lentinan from L. edodes (6) and polysaccharide K from Coriolus versicolor (92), have shown promising effects in clinical trials in conjunction with conventional therapy, and their effects were suggested to be mainly through improvement of immune function. Although these are all promising results, there is little evidence in the literature on direct anti-tumorigenic effects through activation of macrophages by fungal polysaccharides. Because TAMs are attractive targets for cancer immunotherapy $(66,73)$, and NO seems to be important for their direct antitumorigenic effects $(76,93)$, it would be worthwhile investigating such effects from fungal polysaccharides, including polysaccharides isolated from I. obliquus. 


\section{AIMS OF THE THESIS}

The overall aim of this thesis was to isolate and chemically characterize compounds from $I$. obliquus and to investigate the immunomodulating properties of these compounds. According to the literature, extracts from I. obliquus could display either pro- or anti-inflammatory activities, and one aim of the thesis was therefore to clarify which compounds could be causing the specific activities. Another central aim was to increase knowledge about the immunological activities of the compounds by using new approaches and experimental setups in order to learn more about the mechanisms of action by the isolated compounds.

Specific aims were as follows:

- To isolate, purify and chemically characterize polysaccharides from I. obliquus, and identify central differences between water-extracted and alkali-extracted polysaccharides (paper I).

- To investigate the potential of the isolated polysaccharides to activate macrophages into a pro-inflammatory and/or tumoricidal phenotype (paper II).

- To identify which pattern recognition receptors were responsible for the macrophage activation by the isolated polysaccharides (paper II).

- To isolate triterpenoids from I. obliquus, preferably novel triterpenoids or triterpenoids that had not been found in I. obliquus previously (paper III).

- To screen the isolated triterpenoids for a variety of in vitro bioactivities including immunological activities, in order to find new usage for "old" compounds (paper III).

- To isolate and chemically characterize melanin pigments from I. obliquus, and investigate their immunological properties (paper III).

- After it was discovered that some triterpenoids and a melanin pigment interacted with the complement cascade, a new aim became to discover if this interaction was inhibitory or stimulatory, and which complement pathways were involved (paper III). 


\section{SUMMARY OF PAPERS}

\section{Paper I}

\section{Structural characterization of bioactive heteropolysaccharides from the medicinal fungus Inonotus obliquus (Chaga)}

The aim of this paper was to perform a comprehensive characterization of polysaccharides isolated from I. obliquus. Although a few papers describing the monomeric composition and molecular weights of polysaccharides from I. obliquus existed in the literature, satisfactory characterization of the polysaccharides, including anomeric configuration and linkage analysis, have not been reported. Further, because I. obliquus water-extracts have been used in traditional medicine against gastrointestinal disorders, it would be relevant to investigate the structure and biological activities of polysaccharides from such extracts. To isolate polysaccharides, finely ground fungal material from interior (IOI) and exterior (IOE) parts of I. obliquus were preextracted using DCM and $\mathrm{MeOH}$, followed by water- and alkali extraction, ethanol precipitation and dialysis. This extraction scheme yielded two water extracts (IOI-W and IOEW) and two alkali extracts (IOI-A and IOE-A) rich in polysaccharides. From the water extracts, neutral (IOI-WN and IOE-WN) and acidic (IOI-WAc and IOE-WAc) polysaccharide fractions were obtained after anion-exchange chromatography. IOI-WAc was fractionated further using a size-exclusion chromatography (SEC) column, to give three final acidic polysaccharide fractions (IOI-WAcF1, IOI-WAcF2 and IOI-WAcF3). From the alkaline extracts, only IOI-A contained satisfactory amounts of polysaccharides, and this extract was purified using SEC, yielding the polysaccharide fraction IOI-A1.

All polysaccharides were analyzed for their monosaccharide composition using gas chromatography (GC), and linkage analysis was carried out using GC-MS after methylation of the polymers. In addition, anomeric configuration and other structural details were determined using NMR spectroscopy. Molecular weights and size distribution of the fractions was determined by SEC-MALLS. Smith degradation was carried out to determine the relationship between different parts of the polymer. All water-extracted polysaccharide fractions were heterogeneous and contained several types of sugars. The main structural motif in all fractions was $(1 \rightarrow 3) /(1 \rightarrow 6)$ - $\beta$-glucose (Glc). Substantial amounts of $(1 \rightarrow 6)$ - $\alpha$-galactose $(\mathrm{Gal})$, some of which was $3-O$-methylated, was present in most fractions. In addition, $(1 \rightarrow 4)-\beta$-xylose (Xyl), 
as well as $\alpha$-mannose (Man), $\alpha$-rhamnose (Rha) and glucuronic acid (GlcA) were also parts of the fractions in varying amounts. The neutral polysaccharide fractions had molecular weights of 60-73 kDa, whereas the acidic polysaccharide fractions had substantially lower molecular weights (10-31 kDa). The acidic fractions differed from the neutral ones due to the presence of $(1 \rightarrow 4)$ - $\alpha$-galacturonic acid (GalA). Further, Smith degradation of the water-soluble fractions indicated that the polymers had a backbone of Glc with $(1 \rightarrow 3)$-linked stretches 5-7 monomers long, possibly separated with $(1 \rightarrow 6)$-linkages in between. Interestingly, no major structural differences between the IOI and IOE fractions were found. The alkaline polysaccharide fraction IOI-A1 consisted mainly of $(1 \rightarrow 3)-\beta$-Glc with presence of some $(1 \rightarrow 6)-\beta$-Glc and $(1 \rightarrow 4)-\beta-X y l$. This fraction had a much higher molecular weight than the water-extracted fractions $(>450$ $\mathrm{kDa}$ ), and was particulate (i.e. non-water-soluble).

The polysaccharide fractions were screened for in vitro immunological activity by their ability to induce nitric oxide (NO) secretion from the two murine cell lines J774.A1 (macrophage cell line) and D2SC/1 (dendritic cell line). When used at a concentration $100 \mu \mathrm{g} / \mathrm{mL}$ in combination with IFN- $\gamma$, all fractions managed to induce NO production in the D2SC/1 cells, detected using the Griess assay to measure nitrite levels in the cell supernatants. In the J774.A1 cell line, all polysaccharide fractions except IOI-A1 induced NO production. The fractions were analyzed for the presence of LPS by GC/MS, and it was revealed that IOE-WAc contained some LPS $(0.16 \%)$, whereas the rest of the fractions tested negative for LPS.

Thus, it was concluded that complex and heterogeneous polysaccharides were successfully isolated from I. obliquus, and that they were able to activate macrophages and dendritic cells in vitro. 


\section{Paper II}

Polysaccharides from the fungus Inonotus obliquus (Chaga) activate macrophages into a tumoricidal phenotype via interaction with TLR2, TLR4 and Dectin-1a

In paper I, it was observed that some of the I. obliquus polysaccharide fractions were able to induce nitric oxide production in macrophage and dendritic cell lines. This was a clear sign that they were immunologically active, and of interest to be explored further. Bone marrow-derived macrophages (BMDMs) were isolated from BL6 mice, and a number of different experiments were conducted to investigate the immunological effects of the polysaccharides. First, the ability to induce NO production in BMDMs by I. obliquus polysaccharides was measured using the Griess assay and by quantifying iNOS mRNA levels from the cells after treatment. Further, IL-6 and TNF- $\alpha$ concentration in BMDM supernatants were quantified using Luminex technology. To investigate if the polysaccharides also activated human cells, human monocytederived macrophages were included in the cytokine detection assay in addition to BMDMs. In addition, a growth inhibition assay using Lewis lung carcinoma cells in co-culture with wild type and TLR4 knockout BMDMs was carried out to investigate if activation by I. obliquus polysaccharides induced a tumoricidal phenotype in the BMDMs (Figure 10). 
A

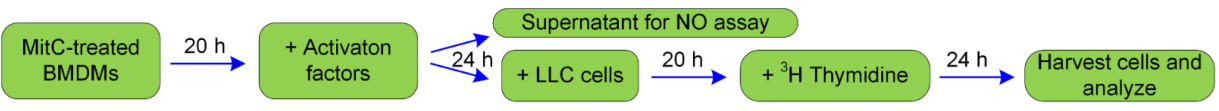

B

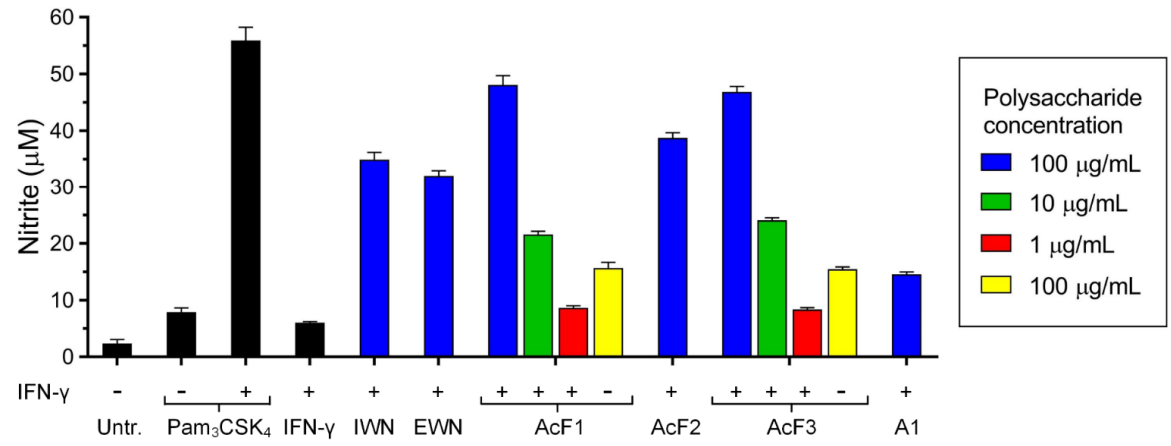

C

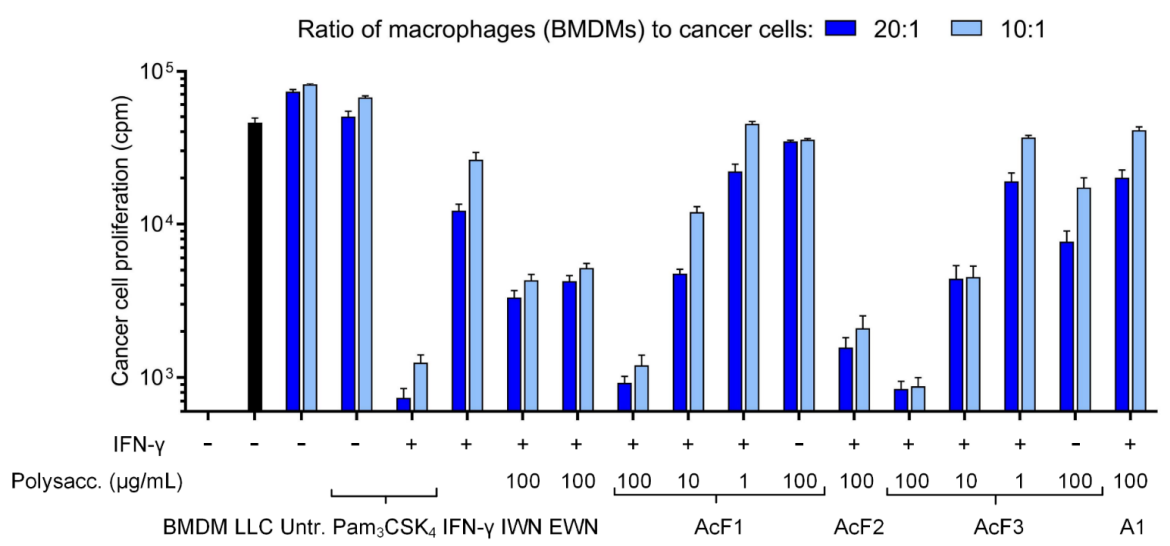

Figure 10. Polysaccharides from I. obliquus in combination with IFN- $\gamma$ inhibit cancer cell growth by activating macrophages. A) The experimental setup: wild-type BMDMs $\left(6 \times 10^{4}\right.$ cells/well or $3 \times 10^{4}$ cells/well for 20:1 and 10:1 ratios, respectively) were incubated with mitomycin $\mathrm{C}$ for $2 \mathrm{~h}$, and then cultivated for $20 \mathrm{~h}$ before treatment with polysaccharides and/or IFN- $\gamma$ for $24 \mathrm{~h}$. Then, $100 \mu \mathrm{L}$ cell medium was removed and analysed for NO before Lewis lung carcinoma (LLC) cells $\left(3 \times 10^{3}\right.$ cells/well) were added to the well containing macrophages, giving two different ratios between macrophages and cancer cells (20:1 and 10:1). After $20 \mathrm{~h}$, radiolabelled thymidine was added and the cells were incubated for another $24 \mathrm{~h}$ before inhibition of cancer cell growth was measured. B) NO concentration in the cell medium was measured using the Griess assay. The polysaccharide concentration was 100 $\mu \mathrm{g} / \mathrm{mL}$ except for AcF1 and AcF3, which were tested in concentrations 1, 10 and $100 \mu \mathrm{g} / \mathrm{mL}$. All samples were tested alone and in combination with IFN- $\gamma(20 \mathrm{ng} / \mathrm{mL}) . \mathrm{Pam}_{3} \mathrm{CSK}_{4}(100 \mathrm{ng} / \mathrm{mL})$ and IFN- $\gamma(20 \mathrm{ng} / \mathrm{mL})$ were used as controls. C) Cancer cell growth inhibition was measured using radiolabelled thymidine, expressed as radioactive counts per minute (cpm) values. The polysaccharide concentration was $100 \mu \mathrm{g} / \mathrm{mL}$ except for AcF1 and AcF3, which were tested in concentrations 1, 10 and $100 \mu \mathrm{g} / \mathrm{mL}$. All samples were tested alone and in combination with IFN- $\gamma(20 \mathrm{ng} / \mathrm{mL})$. Pam ${ }_{3} \mathrm{CSK}_{4}(100 \mathrm{ng} / \mathrm{mL})$ and IFN- $\gamma(20 \mathrm{ng} / \mathrm{mL})$ were used as controls. Untreated control (Untr.) consisted of co-cultured, non-activated BMDMs and LLC cells. Three independent experiments were performed, and a representative experiment is shown using average values \pm SD from technical triplicates. 
In all above-mentioned experiments, the acidic polysaccharide fractions IOI-WAcF1 (AcF1) and IOI-WAcF3 (AcF3) were the most active. Notably, AcF3 induced potent cytokine production in both human and murine macrophages. In the murine macrophages, activation by AcF3 induced comparable levels of IL-6 $(\sim 2 \mathrm{ng} / \mathrm{mL})$ and TNF- $\alpha(\sim 1 \mathrm{ng} / \mathrm{mL})$ to the positive control $\mathrm{Pam}_{3} \mathrm{CSK}_{4}$. In the human macrophages, $\mathrm{AcF} 3$ induced higher levels of IL-6 than $\mathrm{Pam}_{3} \mathrm{CSK}_{4}(\sim 15 \mathrm{ng} / \mathrm{mL}$ vs $\sim 7 \mathrm{ng} / \mathrm{mL}$, respectively), and induced comparable levels to LPS. For TNF- $\alpha$, AcF3 induced similar levels as Pam $_{3} \mathrm{CSK}_{4}$ and LPS ( $\left.15 \mathrm{ng} / \mathrm{mL}\right)$.

AcF1 and AcF3 induced strong cancer cell growth inhibition through activation of BMDMs, which correlated with NO production. Interestingly, IOI-A1 (A1) was not active in any of the assays, demonstrating that only the water-soluble I. obliquus polysaccharides with a high complexity were able to activate the macrophages. To exclude that LPS contamination was causing the activation, TLR4 knockout BMDMs were isolated, and used for the growth inhibition assay. The results showed that the I. obliquus polysaccharides, in particular AcF1 and $\mathrm{AcF} 3$, retained their activity when using these cells, thus confirming the immunological activity of the polysaccharides.

To identify receptors that were causing the macrophage activation, HEK-Blue ${ }^{\mathrm{TM}}$ reporter cells lines transfected with either human TLR4, human TLR2 or human Dectin-1a were used. Surprisingly, AcF3 and AcF1 were able to activate TLR4 in a dose-dependent manner. However, much higher concentrations were needed for activation compared to LPS. At a concentration of $100 \mu \mathrm{g} / \mathrm{mL}$, the activation by AcF1 and AcF3 was comparable to LPS (10 $\mathrm{ng} / \mathrm{mL}$ ). In addition, the acidic polysaccharides AcF1, AcF2 and AcF3 activated TLR2, with comparable potency as zymosan, which is a known TLR2 agonist. Interestingly, Dectin-1a was activated by IOI-A1 (A1), IOI-IWN (IWN) and IOE-WN (EWN) and to a smaller extent by $\mathrm{AcF} 1, \mathrm{AcF} 2$ and AcF3. The potency of the I. obliquus polysaccharides against Dectin-1a was weaker than zymosan (a particulate $\beta$-glucan) but was comparable to laminarin (a soluble $\beta$ glucan). These findings, combined with results from the TLR4 knockout cells suggest that TLR2 is the most important receptor for macrophage activation by I. obliquus polysaccharides, but that both TLR4 and Dectin-1a might play additional roles in activation (Figure 11). Thus, it can be concluded that I. obliquus polysaccharides, notably AcF1 and AcF3, were able to induce a pro-inflammatory, tumoricidal phenotype in murine macrophages, and that this likely happened through activation of multiple receptors, here demonstrated via TLR2, TLR4 and Dectin-1a. 


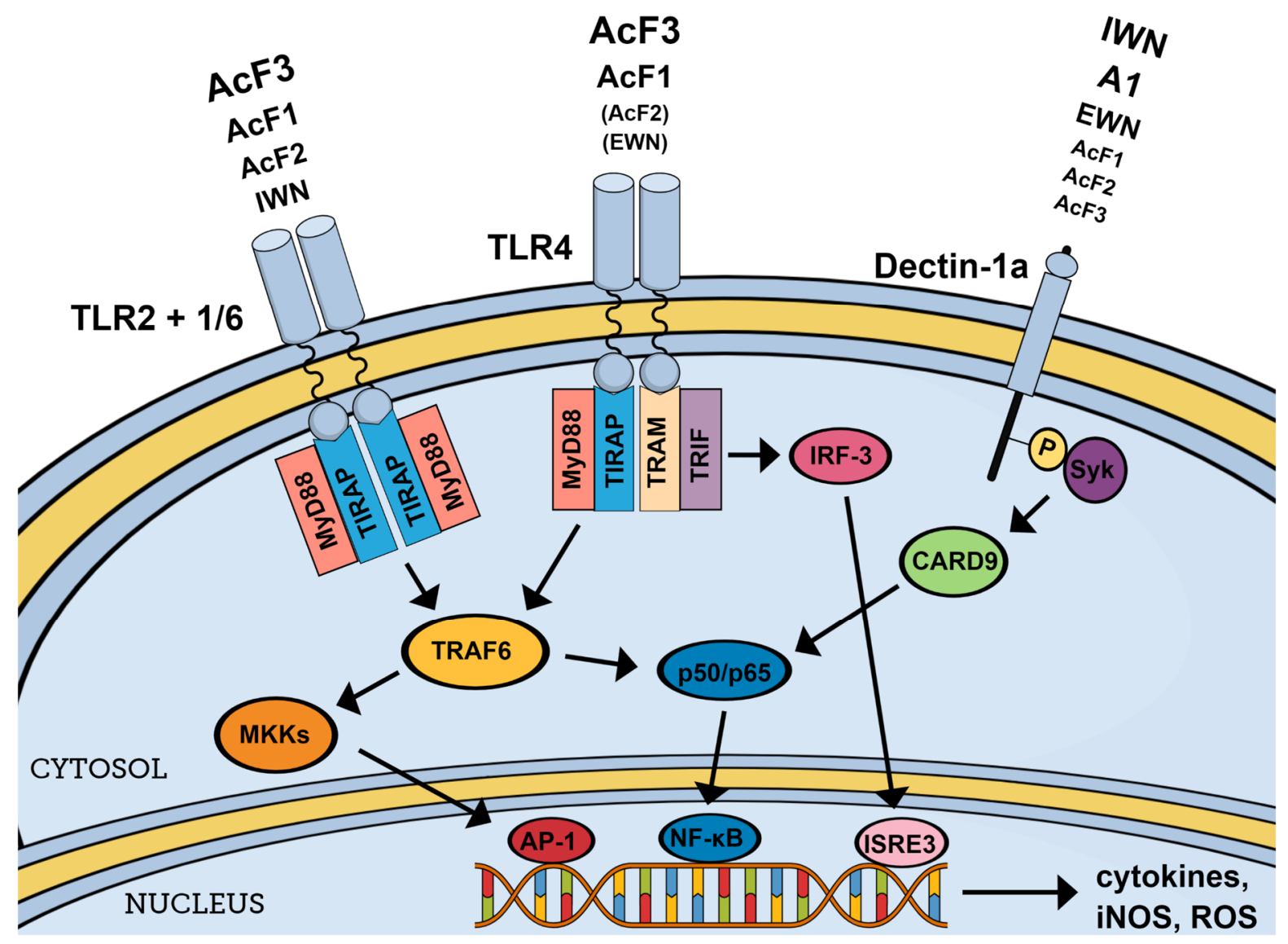

Figure 11. I. obliquus polysaccharides, in particular AcF1 and AcF3, were able to activate TLR2 and TLR4. In addition, IWN, A1, EWN and to some extent AcF1, AcF2 and AcF3 activated Dectin-1a. The activation of these receptors could trigger an intracellular signalling cascade in macrophages, leading to activation of the transcription factors AP-1, NF-KB and ISRE3, leading to production of pro-inflammatory cytokines, NO and other reactive oxygen species (ROS) in the macrophages. This could again lead to functional outcomes in the macrophages such as killing of cancer cells. 


\section{Paper III}

\section{Bioactive triterpenoids and water-soluble melanin from Inonotus obliquus (Chaga) with immunomodulatory activity}

In paper I, it was discovered that melanin was one of the main components in the I. obliquus water extracts. This warranted further investigation of this pigment, especially since there were no reported biological activities from water-soluble I. obliquus melanin in the literature. Further, because I. obliquus water-extracts have been used in traditional medicine against gastrointestinal disorders, it would be worthwhile investigating the immunological properties of the melanin pigments. In addition, many triterpenoids from I. obliquus have been chemically characterized before, but although their cytotoxic properties have been investigated, there are not many reports on immunological properties of these compounds. First, a melanin fraction was purified and isolated from the IOI-W extract described in paper I. The fraction was chemically characterized by various methods. Combustion analysis revealed an absence of nitrogen - this was indicative of the pigment not belonging to the eumelanin class. The pigment was also compared with a eumelanin from Sepia officinalis by using HPLC to analyze degradation products from both types of melanin. It was concluded that the I. obliquus pigment was fundamentally different from the $S$. officinalis eumelanin sample, and that it probably was a DHN-type melanin pigment, which are commonly found in fungi. Interestingly, the melanin pigment seemed to be covalently bound to carbohydrates. This was probably the reason for the water-solubility of the pigment, because melanin pigments by themselves are known to be insoluble in water. In addition to melanin, six triterpenoids were isolated from the DCM extracts from the interior and exterior parts of the fungus by extensive chromatographic purification using e.g. HPLC and TLC, before the compounds were identified using NMR spectroscopy and MS. The triterpenoids isolated were as follows: inotodiol, betulinic acid, betulin, betulin-3-Ocaffeate, trametenolic acid and 3ß-hydroxy-8,24-dien-21-al.

All triterpenoids as well as the melanin pigment were screened for various bioactivities, including complement-fixating activity, anti-inflammatory activity by reduction of LPSinduced NO production in macrophages, and cytotoxicity using the lung carcinoma cell line H460 and the colorectal adenocarcinoma cell line HT29-MTX. In addition, the ability to inhibit the enzymes xanthine oxidase, 15-lipoxygenase and acetylcholinesterase as well as antimicrobial and antioxidant activities were investigated. Interestingly, the melanin pigment as well as inotodiol and 3ß-hydroxy-8,24-dien-21-al were highly active in the complement- 
fixating assay, with 3 $\beta$-hydroxy-8,24-dien-21-al standing out, having an $\mathrm{ICH}_{50}$ value $=1.8 \pm$ $0.4 \mu \mathrm{M}$ (Figure 12). Notably, the other triterpenoids were completely inactive in this assay even at concentrations $>100 \mu \mathrm{M}$. This suggests that some specific pharmacophore was present in $3 \beta$-hydroxy-8,24-dien-21-al and inotodiol that made them interact with the complement cascade. Follow-up experiments were carried out by varying the incubation times of compounds with complement-containing human serum, revealing that the melanin pigment had an inhibitory effect on the complement cascade whereas the triterpenoids showed an activating effect. Further, using a commercial ELISA complement pathway detection kit, it was discovered that the melanin pigment inhibited all the three complement pathways (classical, lectin and alternative), whereas 3 $\beta$-hydroxy-8,24-dien-21-al and inotodiol activated the classical and lectin pathways.

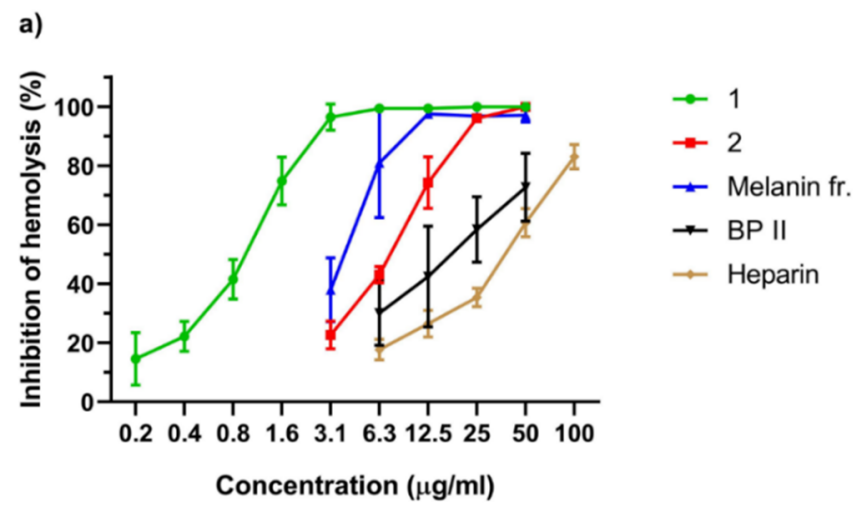

b)

Interaction with complement pathways

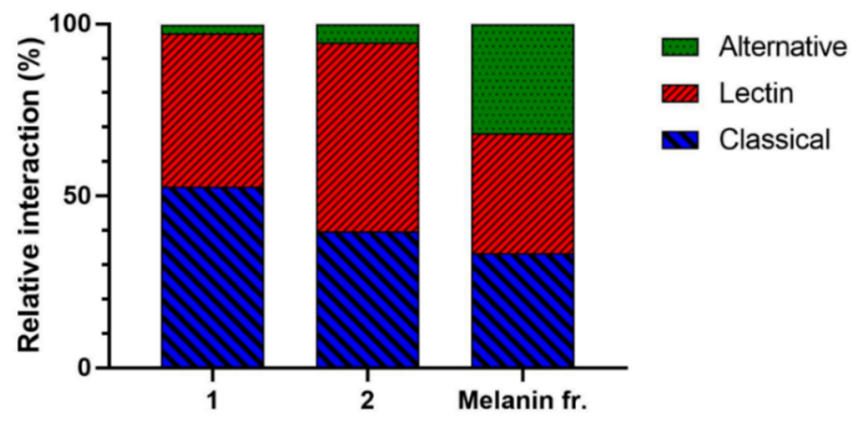

Figure 12. An I. obliquus melanin fraction and the triterpenoids 3 $\beta$-hydroxy-8,24-dien-21-al (1) and inotodiol (2) interact strongly with human complement. a) Inhibition of complement-induced hemolysis of sheep erythrocytes. Compounds were incubated with human serum for $30 \mathrm{~min}$ prior to addition of erythrocytes. BPII (a polysaccharide isolated from $B$. petersianum) and heparin were used as positive controls. Results are expressed as means $\pm \operatorname{SD}(n=3)$. b) Interaction with the classical, lectin and alternative pathways of the complement cascade. 
The melanin fraction and betulin-3-O-caffeate showed modest activity in the anti-inflammatory assay $\left(\mathrm{IC}_{50}\right.$ values $=24.1 \pm 7.9 \mu \mathrm{g} / \mathrm{mL}$ and $17.6 \pm 3.7 \mu \mathrm{M}$, respectively), being able to reduce NO concentration in a dose-dependent manner after stimulation of macrophages with LPS and IFN- $\gamma$. Further, betulinic acid, betulin and inotodiol had potent anti-proliferative activity against the colon adenocarcinoma cells line HT29-MTX ( IC $_{50}$ values $=0.8 \pm 0.3 \mu \mathrm{M}, 1.6 \pm 0.4 \mu \mathrm{M}$ and $3.8 \pm 0.8 \mu \mathrm{M}$, respectively) and the lung carcinoma cell line NCI-H460 ( IC $_{50}$ values $=2.1 \pm 0.5$ $\mu \mathrm{M}, 2.8 \pm 0.4 \mu \mathrm{M}$ and $4.7 \pm 1 \mu \mathrm{M}$, respectively) as measured using the MTT assay. All compounds were inactive in the enzyme-inhibition assays and in the anti-microbial assay used in this study. Taken together, the results demonstrate the anti-inflammatory and immunomodulating effects of I. obliquus melanin and triterpenoids. 


\section{GENERAL DISCUSSION}

In this thesis, immunological activities of isolated and chemically characterized polysaccharides, triterpenoids and melanin from I. obliquus have been investigated. Overall, the results showed that I. obliquus compounds exhibited either inhibitory or activating effects on macrophages and on complement proteins. Perhaps not surprisingly, the biological activity of the compounds was dependent on to which class of natural product they belonged. Watersoluble polysaccharide fractions consisting of $\beta$-Glc along with $\alpha$-Gal, $\alpha$-GalA, $\alpha$-Man and $\beta$ $\mathrm{Xyl}$ were shown to induce pro-inflammatory responses in macrophages by being recognized by pattern recognition receptors. Conversely, an isolated melanin fraction from the same water extract inhibited a pro-inflammatory macrophage phenotype in a similar experimental setup by reducing NO in the supernatant after activation with LPS and IFN- $\gamma$, as did the triterpenoid betulin-3-O-caffeate. Further, the melanin pigment was able to inhibit the complement cascade whereas the triterpenoids inotodiol and 3 $\beta$-hydroxy-8,24-dien-21-al activated the same cascade.

Some of the results presented in this thesis demonstrate that extracts from plants and fungi are complex mixtures of compounds that have the potential of negating each other's effects, which is often the case for crude extracts (94). This was most evident for the water extracts that were used to obtain polysaccharides and melanin. Interestingly, when macrophages were treated with the I. obliquus "crude polysaccharide extract" (IOI-W, paper I) that was prepared by ethanol precipitation and dialysis of the water extract, NO could not be detected in the supernatant, even when the extract was used in combination with IFN- $\gamma$ (results not shown). Analysis showed that this extract contained mainly polysaccharides and melanin, thereby suggesting that melanin was the dominating part of the extract, at least for this particular experimental setup. It has previously been shown that many pathogenic fungi utilize melanin as a way of escaping the host immune response (53). One possible explanation is that melanin is located in the outer part of the fungal cell wall, whereas the more immunologically active $\beta$-glucans are located closer to the cell membrane (Figure $2 \mathrm{c}$ ). Thus, the $\beta$-glucans would be more difficult to detect by PRRs on macrophages because they hidden below the other cell wall components including melanin. However, from the macrophage experiments using IOI-W, it seems evident that melanin was capable of negating the activity of the pro-inflammatory polysaccharides even without being part of a cell wall. This could be explained by an antioxidant activity of melanin that would reduce NO levels in the cell supernatant, meaning that the polysaccharides in the extract initially 
induced NO production and that NO was subsequently neutralized by melanin when secreted into the cell medium. Therefore, to investigate the anti-inflammatory effects of melanin in more detail, it should be attempted to re-produce the experimental setup by quantifying proinflammatory cytokines such as TNF- $\alpha$ instead of free radicals like NO (or nitrite). However, IOI-W was included in the HEK-Blue ${ }^{\mathrm{TM}}$ reporter cell line assays, and strikingly, neither TLR2 or TLR4 were activated by the extract, suggesting that melanin efficiently "cloaked" the polysaccharides from their target receptors (results not shown). The findings are quite relevant to the role of I. obliquus as a beverage, because the water extract used in this thesis is similar to the ones used in traditional medicine and similar to products commercially available in health stores today. The results presented in this thesis suggest that the effects these extracts would have on the gastrointestinal system are likely to be anti-inflammatory rather than proinflammatory. However, several factors could influence the in vivo effects of extracts, such as bioavailability of the compounds and their interaction with the gut microbiota. The effects of $I$. obliquus extracts and compounds on the gastrointestinal system should be therefore be explored further in order to conclude anything in this regard.

\section{Interpretation of the polysaccharide structure}

One important aim of this thesis was to isolate and characterize polysaccharides and identify their detailed chemical composition. A few research groups have reported the monosaccharide composition and molecular weights of water-soluble polysaccharides from I. obliquus $(16,17$, 35). In addition to the more commonly found fungal sugars Glc and Man, it seems evident that I. obliquus polysaccharides from water extracts can contain Gal, Xyl, Rha, Ara and GalA in high amounts. The polysaccharide fractions isolated in this thesis consisted of several different monomers with many types of linkages, and with both $\alpha$ - and $\beta$ - anomeric configurations. Apart from the $(1 \rightarrow 3 / 1 \rightarrow 6)$ - $\beta$-Glc motif, the most common structural motifs detected in the isolated polysaccharide fractions were $(1 \rightarrow 6)-\alpha-G a l,(1 \rightarrow 4)-\alpha$-GalA and $(1 \rightarrow 4)-\beta-X y l$, as well as $(1 \rightarrow 3)$ and $(1 \rightarrow 2)-\alpha-$ Man. Interestingly, the Gal units were partially $O$-methylated at $\mathrm{C} 3$, and this characteristic had not been described in I. obliquus before. There are a few reports on 3-Omethylated Gal in some other fungal species such as fungi from the Phellinus and Pleutotus genera $(95,96)$. Although a concrete biological function of these methylated sugars has not yet been identified, it seems that the specific 3-O-methylation of Gal is evolutionary conserved across several species, and it is therefore likely that it has some important biological function in the fungi. One possible explanation is that methylation makes the polymers more lipophilic, 
and make them interact more easily with hydrophobic parts of proteins in the cell wall. Indeed, it has been suggested that methylation of sugars are involved in recognition of certain undefined structures (97). However, the role of such methylation remains speculative, and experimental evidence is needed to identify the concrete biological roles of methylated sugars in the fungal cell wall.

The structures of the isolated I. obliquus polysaccharide fractions were characterized by several methods, such as GC-MS linkage analysis, NMR spectroscopy, SEC-MALLS and Smith degradation. Based on the collective knowledge obtained from the various experiments, structural compositions of the polymers were suggested, and these are presented in Figure 13.

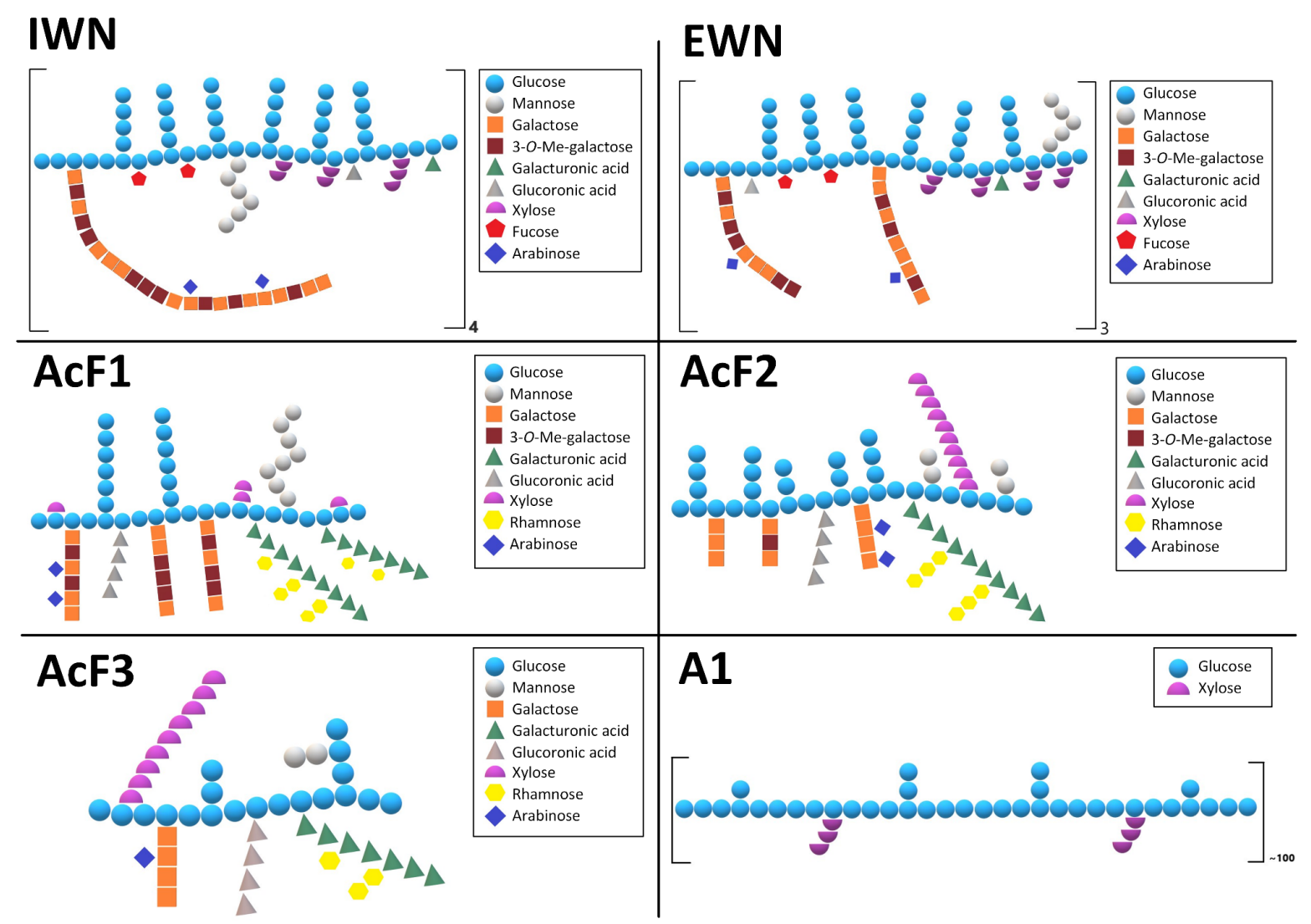

Figure 13. Suggested structures and short names for the polysaccharides isolated from I. obliquus.

The structures in Figure 13 are hefted with a significant degree of uncertainty. One of the most challenging tasks when characterizing polysaccharides is to determine how the different parts of the polymers are connected. In the case of the I. obliquus polysaccharide fractions presented in this thesis, it was difficult to find any direct proof of direct linkage between the different monomers, such as a connection between $\beta$-Glc and $\alpha$-Gal. Therefore, it might be that the 
polysaccharide fractions consisted of more than one type of polymer. However, there are several indications that this was not the case. First, the anomeric signals observed in HSQC spectra from the fractions were quite stretched out, indicating that many of the monomers were bound to other sugars, which would change their shift values slightly. Further, in order to detect couplings between the separate monomers within the polymer chain using NMR spectroscopy, the polymer must contain repetitive motifs with many couplings between the monomers, as is the case with e.g. galactomannans (98). In these cases, the couplings could be detected using e.g. HMBC experiments. Conversely, if different parts of a given heteropolysaccharide were to be linked together at only a few monomers, NMR signals from these monomers would be too weak to detect because they would make up a tiny percentage of the total signals, thus NMR spectroscopy would not be sensitive enough to detect the signals. This might be the case for the I. obliquus polysaccharides.

When purifying the water-soluble polysaccharide fractions, several types of SEC columns with different pore sizes were tested in order to obtain the best possible separation and purification. However, we were not able to separate the different types of sugars from each other. If indeed there were several types of homogenous polysaccharides in the fractions, e.g. pure $\beta$-glucans and pure $\alpha$-galactans, it is unlikely that they all were of identical sizes so that they could not be separated using any of the SEC columns. Further, SEC-MALLS analysis of the fractions revealed that IWN, AcF1, AcF2 and AcF3 had a narrow size-distribution, indicating that the fraction contained polymers of highly similar sizes. In addition, several master students working in our group isolated polysaccharides from I. obliquus collected at different sites than the one used in this thesis. The monosaccharide composition of isolated polysaccharide fractions in these projects were almost identical to the ones presented here. Although these arguments cannot completely rule out the possibility of a mixture of polymers, the results collectively suggest that the fractions contained heteropolysaccharides consisting of many types of sugars.

There are some logical explanations for the arguments presented above. As seen in Figure 2 in the Introduction, fungal cell wall polysaccharides can be cross-linked at one or two sites for each polymer to increase structural rigidity, and this might be the case for the I. obliquus polysaccharides as well $(23,99)$. Further, while cell wall polysaccharides usually serve stabilizing functions in the fungal cell wall, they may also be involved in communication with the local environment, and their structures can change with time depending on the life cycle of the cell, leading to more complex structural compositions of the polymers (23). Although polysaccharides are synthesized enzymatically, their structures are not encoded specifically by 
genes (as opposed to proteins), and therefore it is possible that they to some extent are "randomly" assembled or coupled, especially if there is no selective evolutionary pressure toward specific structural motifs. This could explain some of the variation and complexity observed for many fungal polysaccharides, such as the ones isolated from I. obliquus in this thesis. Lastly, pectic polysaccharides from plants are polymers well documented to be highly complex and heterogeneous (100), suggesting that this is a common phenomenon in nature and could thus be the case for fungal polysaccharides as well.

There are a few ways of observing direct experimental evidence for couplings in heteropolysaccharides. However, for the most complex polysaccharides, the detailed relationship between the monomers can be quite difficult to unravel. The usage of enzymes to degrade parts of the polymer can be used to identify the relationship between the polymers' different parts. However, such enzymes are usually quite specific for one type of monomer, e.g. the cleavage of $(1 \rightarrow 3)-\beta$-glucans is carried out by a $(1 \rightarrow 3)-\beta$-glucanase. Therefore, heteropolysaccharides with different sugars and branching points probably need a combination of different enzymes in order to be degraded (101). It can be more complicated to utilize enzymes for degradation of fungal polysaccharides than for plant polysaccharides like pectin. This is mainly because the specific enzymes that degrade fungal polysaccharides are much less characterized than their plant counterparts, and commercial availability of enzymes that are specific toward a given fungal species can be virtually non-existent. It was attempted to use enzymatic degradation for the I. obliquus polysaccharides isolated in this thesis, and several types of enzymes were tested. The enzymes tested were from Megazyme, and included: exo$(1 \rightarrow 3)-\beta$-glucanase $+\beta$-glucosidase (E-EXBGOS), $\alpha$-galactosidase (E-AGLAN), exo-polygalacturonase (E-EXPGA) and endo- $(1 \rightarrow 4)-\beta$-xylanase (E-XYTR3). The enzymes were tested alone and in various combinations. However, the enzymes were not successful in changing the monomeric composition of the fractions, and it might be that other combinations of enzymes are required for successful degradation of the I. obliquus polysaccharides. In addition to enzymatic degradation, partial hydrolysis using a weak acid might be another way to increase knowledge about the polysaccharide structure (102). It would be worthwhile to investigate the fine structure of the I. obliquus polysaccharide fractions further, both to reveal the true nature of the polymers and to reveal which parts of the polysaccharides are causing the biological activities. 


\section{Structure-activity relationship of the polysaccharide fractions and interaction with pattern recognition receptors}

The results presented in paper II revealed that the acidic polysaccharide fractions AcF1 and AcF3 were the most potent in the macrophage activation assays. Furthermore, they were devoid of LPS contamination and were able to activate TLR2, TLR4 and to some extent Dectin-1a. The main difference between the acidic and the neutral fractions was the presence of $(1 \rightarrow 4)-\alpha-$ GalA in the acidic polysaccharides (13-18\% of total carbohydrate) (Figure 13). It therefore seems likely that this motif was important for the activation of macrophages and for the TLR2and TLR4-binding capabilities. Interestingly, citrus pectin containing mainly $(1 \rightarrow 4)-\alpha-\mathrm{GalA}$ has previously been shown to bind both TLR2 and TLR4 (103). One study found that citrus pectin containing de-esterified GalA was able to inhibit activation of TLR2/1 due to negative charges on the polymer associating with positively charged areas of TLR2, but that TLR2/6 activity was unaffected (104). These findings suggest that GalA is able to bind TLR2 but is not necessarily responsible for activation of the receptor. Thus, it might be that other parts of the $I$. obliquus polysaccharides were important for TLR activation, requiring GalA in addition to other structural motifs present in AcF1 and AcF3, such as $\beta$-Glc.

The neutral fraction IWN activated TLR2 to some extent, while EWN activated TLR4 to some extent, although these fractions did not contain GalA, further suggesting that some structural motif other than GalA was important for the receptor interaction. IWN and EWN contained more of $\beta$-Glc than the acidic fractions ( $\sim 50 \%$ in neutral fractions vs $34-43 \%$ in the acidic fractions), but had lower activity in the macrophage activation experiments. Therefore, it seems likely that $\beta$-Glc was not solely responsible for the immunological activity. The low amounts of Gal and 3-O-Me-Gal in AcF3 (9\% vs $20 \%$ for AcF1) and low amounts of Xyl in AcF1 (5 $\%$ vs $19 \%$ in AcF3) suggest that these monomers are not crucial for the activation of TLR2 and TLR4. Further, the high amounts of Man in AcF1 and AcF2 compared to AcF3 suggests that neither this monosaccharide is the main cause of the biological activity. Finally, it is likely that some of the differences in activity are due to variations in the three-dimensional structure of the polysaccharides, which is known to be important for interaction with many PRRs (105). This could explain why AcF2 appeared less active at binding TLR2 and TLR4 than AcF1 and AcF3. According to GC-MS data, AcF2 exhibited a higher degree of side-chains than AcF1 and AcF3, which could affect the accessibility of GalA. Collectively, the data suggests that the presence 
of $(1 \rightarrow 4)-\alpha-G a l A$ combined with a highly complex structural motif were important features for activating TLR2 and TLR4 by I. obliquus polysaccharides.

The interaction with Dectin-1a seemed dependent on $(1 \rightarrow 3)$ - and/or $(1 \rightarrow 6)-\beta-G l c$, as is wellestablished in the literature $(34,86)$. IWN, EWN and IOI-A1 were the fractions with the strongest activity toward this receptor, managing to activate Dectin-1a at a concentration of 1 $\mu \mathrm{g} / \mathrm{mL}$, whereas AcF1, AcF2 and AcF3 were only active at concentrations of $100 \mu \mathrm{g} / \mathrm{mL}$. AcF1, AcF2 and AcF3 contained lower amounts of $\beta$-Glc than IWN and EWN. However, because the dominating structural motif in the acidic fractions was still $\beta$-Glc, they should be expected to have comparable levels of Dectin-1a activation as the neutral fractions. It might be that the presence of GalA negatively influenced the activity toward Dectin-1a, for example due to changes in the three-dimensional structure of the polymer, or due to the $\beta$-Glc motif being less exposed to the receptor.

There were some discrepancies between the experiments carried out in the cell lines (paper I) and primary cells (paper II). In paper I, it was concluded that EWN was the most promising of the polysaccharide fractions, in particular in the dendritic cell line D2SC/1. In paper II, the acidic polysaccharides AcF1 and AcF3 were the most active, and the potent activity of the fractions was likely caused by activation of TLR 2 and TLR4. Interestingly, both IWN and EWN induced NO and cytokine production in the BMDMs, but only induced a slight activation of TLR2, TLR4 and Dectin-1a. It therefore seems probable that the activation of the cells by EWN and IWN were caused by activation of some other, yet unidentified receptors. It could be that the cell lines had a different distribution of PRRs than the primary macrophages. Macrophages and dendritic cells are dynamic cell types that change their phenotype and receptors densities depending on signals from their local environment. Further, other PRRs such as CR3 (106), the galectins, DC-SIGN and NLRs are able to recognize fungal polysaccharides (23), and interaction with these receptors by the I. obliquus polysaccharides should be explored further using a combination of reporter cell lines, knockout BMDMs and receptor-blocking antibodies. It has also been shown that TLR2 and TLR4 could work in a synergetic manner with Dectin-1 $(88,89,107)$. Because the water-soluble polysaccharide fractions were shown to activate TLR2, TLR4 and Dectin-1a, the activation could have caused a synergism between the receptors that was sufficient to induce the macrophage activation. 
In summary, the full structure-activity relationship of the polysaccharides remains to be fully discovered, and efforts should be put into resolving this issue by isolating and testing immunological activity of different parts of polymers, in order to discover the mechanisms of action of the polysaccharides and possibly to increase their biological activity.

\section{Impurities in the polysaccharide fractions}

The acidic fractions AcF1, AcF2 and AcF3 contained some traces of phenolic compounds that could have influenced the macrophage activation experiments (4.2, 5.8 and $9.7 \%$ for AcF1, AcF2 and AcF3, respectively). The fractions had a slightly brownish color, and it therefore seemed likely that the color came from melanin or some other phenolic compounds with dark color. It was attempted to remove the phenolics by using various types of SEC columns to separate them from the polysaccharides, in addition to extensive dialysis of the fractions. However, this was not successful, and it was therefore plausible that the phenolics were covalently bound to the acidic polysaccharides. The linkage data obtained by GC-MS revealed no obvious differences in linkage patterns between polysaccharide fraction with and without phenolics, and the GalA units in AcF2 and AcF3 were almost exclusively ( $1 \rightarrow 6)$-linked. If there were some low-molecular weight phenolics covalently bound to the polysaccharides, these probably would have been detected by the linkage analysis. Therefore, it was concluded that the phenolics were of polymeric origin and attached to the polysaccharide chain at only a few sites, which would not be accurately detected by the linkage data. Based on accumulative knowledge of the I. obliquus extracts used in this thesis, it was concluded that this polymer was melanin. It was attempted to remove the melanin pigment by using a weak $\mathrm{HCl}$ solution to precipitate melanin, because I. obliquus melanin has been shown to precipitate at low $\mathrm{pH}(56)$. For the polysaccharide fractions, this was not possible. However, this method was somewhat successful on the crude extracts. Although promising, this method lead to very low yields of pure polysaccharides, and it seemed like a substantial portion of the polysaccharides were precipitated along with melanin, further suggesting a covalent attachment between the two. The covalent attachment was also suggested when the opposite was attempted, to isolate melanin by removal of polysaccharides, as shown in paper III (108). Therefore, the precipitation method to remove melanin needs optimization in order to be useful as a way of obtaining polysaccharides. One possibility is to carry out this method as a first step in the extraction process, using large amounts of fungal material to remove most of the pigment before extraction of water-soluble components. 
Regarding the influence on bioactivity by the melanin pigments, melanin seemed to have an opposite effects of the polysaccharides on macrophage activation, exemplified by the IOI-W extract, and it is therefore unlikely that the melanin pigments would be the cause of the macrophage-activating properties of the acidic polysaccharide fractions. In contrast to IOI-W, which seemed to get its activity from melanin and not from the polysaccharides, the opposite seemed true for $\mathrm{AcF} 1, \mathrm{AcF} 2$ and $\mathrm{AcF} 3$. The higher activity of these acidic fractions compared to the neutral fractions devoid of phenolic compounds demonstrated that the phenolic traces had little influence on the overall activity of the fraction. In addition, AcF2 had a higher phenolic content than AcF1, but was less potent for the activation of the macrophages. However, in the future it should be attempted to remove such traces in order to have as pure polysaccharide fractions as possible.

\section{Considering LPS and lipoprotein contamination in the polysaccharide fractions}

LPS contamination from gram-negative bacteria is a common risk factor when isolating natural products, because such bacteria can be found virtually everywhere, both when collecting plant and fungi and also in the lab (109). This risk becomes particularly evident when testing proinflammatory activity of polysaccharides, since LPS from Escherichia coli can activate macrophages at concentrations of $1 \mathrm{ng} / \mathrm{mL}$ or lower (in-house results). However, not all types of LPS are strong TLR4 agonists, and some are even TLR4 antagonists (110). The polysaccharide fractions from I. obliquus used in this thesis were tested for LPS contamination by using GC-MS to detect the lipid A part of LPS, and one polysaccharide fraction, IOE-WAc, tested positive $(0.16 \%$ LPS). Although included in paper I, this fraction was excluded from the macrophage experiments in paper II. Further, the most potent polysaccharide fractions (AcF1 and AcF3) were able to activate TLR4 KO macrophages strongly, and in the HEK-Blue ${ }^{\mathrm{TM}}$ TLR4 reporter cell line the activity of AcF3 and AcF1 was retained after treatment with the LPS antagonist PMB. Therefore, it was concluded that LPS was not the cause of the activity of the polysaccharide fractions used in this thesis. In addition to LPS, some authors have suggested that plant and fungal extracts are often contaminated with microbial lipoproteins that cause potent activation of TLR2, and that immunological activity often stems from these lipoproteins rather than the isolated plant or fungal polymers $(107,111)$. To rule out the possibility of such a lipoprotein contamination in the I. obliquus polysaccharide fractions, an elemental combustion analysis was performed in order to quantify the presence of nitrogen and carbon in 
the extracts. Presence of nitrogen would indicate lipoprotein contamination since bacterial lipoproteins contain large amounts of nitrogen (112). While one of the analysed I. obliquus ethanol extracts contained significant amounts of nitrogen, none of the water- or alkali polysaccharide extracts contained any traces of this element, strongly suggesting that the polysaccharide fractions did not contain such a contamination (results not shown).

\section{Triterpenoid characterization}

Six triterpenoids were isolated from the I. obliquus DCM extracts (Figure 14), and these were found to have anti-inflammatory, anti-proliferative and complement fixating activities in in vitro assays. Triterpenoids are an interesting and abundant class of natural products. It is thought that triterpenoids have a high probability of being biologically active due to their chemical similarity to some human hormones and sterols (37). Therefore, this class of natural products is promising in the search for biologically active compounds. The first triterpenoid isolated from I. obliquus in this thesis was betulin-3-O-caffeate. This compound had not been isolated from I. obliquus before, and in fact, although more than 50 triterpenoids and sterols have been isolated from I. obliquus throughout the years, none of them has contained a caffeic acid moiety. It was therefore attempted to isolate more compounds similar to betulin-3-O-caffeate from the DCM extracts. However, this attempt was not successful, even when using NMR-guided fractionation to look for the presence of phenolic signals in the triterpenoid-rich fractions. Instead, well-known triterpenoids were isolated in order to continue the investigation of biological activities. One of the reasons for the failed attempt could be the low amounts of fungal starting material in the extraction scheme. It could be that caffeic acid-bound triterpenoids are present in very low amounts in I. obliquus, thus it would require more fungal material in order to isolate them. For example, in a paper by Liu et al (40) they isolated low amounts of several novel triterpenoids from I. obliquus using $22 \mathrm{~kg}$ fungal starting material. In this thesis, only $\sim 200 \mathrm{~g}$ of fungus was used for the DCM extraction, sharply reducing the probability of finding new and rare compounds. Another possibility is that the DCM extracts were too nonpolar to contain triterpenoid-caffeate compounds, since the caffeic acid moiety would increase polarity of the triterpenoids. Therefore, it could be that $\mathrm{EtOH}$ or $\mathrm{MeOH}$ extracts are better starting points to look for such compounds. Ideally, if the goal is to isolate such compounds, it should be attempted to use a higher amount of fungal starting material, a semipolar solvent, and using NMR-guided fractionation to purify fractions from these extracts that 
contain phenolic signals, trans olefinic signals, and signals from triterpenoids (such as from methyl groups).

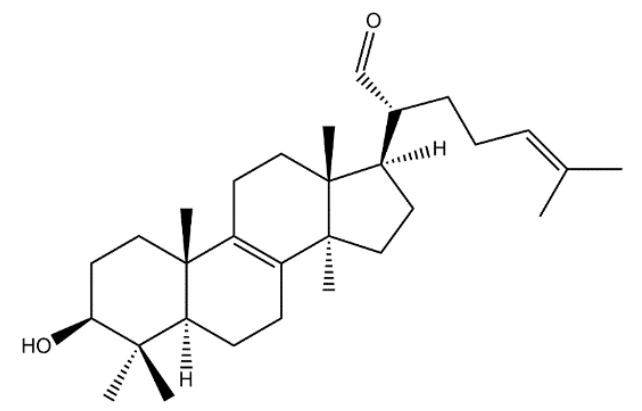

3ß-Hydroxy-8,24-dien-21-al

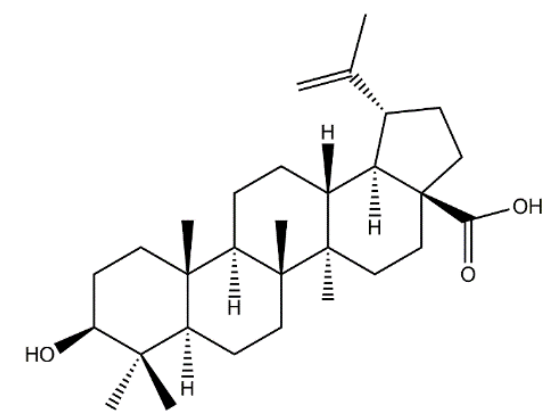

Betulinic acid

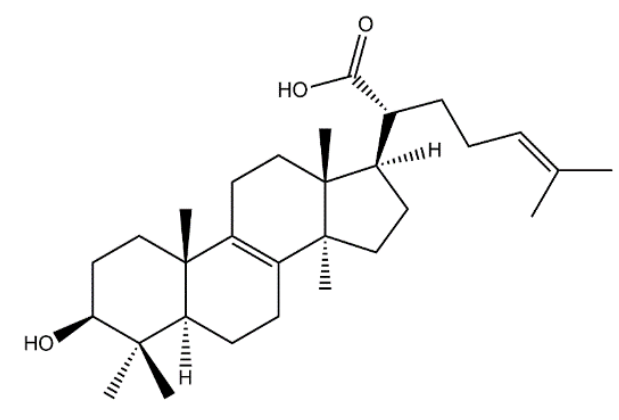

Trametenolic acid



Inotodiol



Betulin



Betulin-3-O-caffeate

Figure 14. Triterpenoids isolated from I. obliquus in this thesis.

Two of the isolated triterpenoids, namely inotodiol and 3ß-hydroxy-8,24-dien-21-al, were active in a complement fixation assay, with $\mathrm{ICH}_{50}$ values of $14.9 \pm 3.9 \mu \mathrm{M}$ and $1.8 \pm 0.4 \mu \mathrm{M}$. The activity of 3 $\beta$-hydroxy-8,24-dien-21-al was of notable potency, having one of the lowest 
$\mathrm{ICH}_{50}$ values ever observed for this assay in our research group. For example, the activity of this compound was 35 times more potent than our positive control BP-II, a pectic polysaccharide with known complement fixating activity $(0.8 \mu \mathrm{g} / \mathrm{mL}$ vs $27.7 \mu \mathrm{g} / \mathrm{mL})$. Interestingly, 3 $\beta$-hydroxy-8,24-dien-21-al has exclusively been isolated from I. obliquus (SciFinder search 08/06-2020). The first report of 33-hydroxy-8,24-dien-21-al was in 1984 (113), and it is found in abundance in I. obliquus, for example being the triterpenoid with the highest yield in this thesis $(23.5 \mathrm{mg})$. Nevertheless, 3ß-hydroxy-8,24-dien-21-al has not yet received a trivial name, as opposed to inotodiol and betulinic acid. This might be due to the absence of interesting biological activities from 3 $\beta$-hydroxy-8,24-dien-21-al, since the compound does not seem to be as cytotoxic to cancer cells as e.g. inotodiol (43). Due to the potent complement-interacting activities of $3 \beta$-hydroxy-8,24-dien-21-al, it might be that the compound now will attract interest from other researchers and will finally be given a name. The complement-interacting activity of inotodiol and 3 3 -hydroxy-8,24-dien-21-al is of noteworthy interest because they seem to elicit a specific and highly structure-dependent activity on the complement cascade. Other classes of compounds, such as polysaccharides and proanthocyanidins, are known to have complement fixating activities as well. However, this is likely to be in a non-specific manner such as polysaccharides being recognized as PAMPs through the lectin pathway (114), and proanthocyanidins being able to precipitate and inactive proteins in a non-specific manner $(115,116)$. In contrast to these class-specific activities, the other triterpenoids tested in this thesis were completely inactive, and even at 50 and $100 \mu \mathrm{M}$ they had zero activity in the complement fixating assay. Additionally, there are almost no publications found in the literature that describes triterpenoids with effects on the complement system, although a few reports on weak complement fixating activities exist, such as from some G. lucidum triterpenoids (117). The general lack of activity and non-existing literature on the matter suggests that a highly specific pharmacophore or moiety exists in 3 $\beta$-hydroxy-8,24-dien21-al and inotodiol. Because these two triterpenoids share the same tetracyclic carbon-skeleton, this skeleton could be important for the complement-interacting activity. However, trametenolic acid, also isolated in this thesis, has a chemical structure similar to $3 \beta$-hydroxy8,24-dien-21-al and inotodiol but was completely inactive in the complement fixating assay. Therefore, further studies should be carried out in order to locate the pharmacophore(s) causing the activity. A good starting place would be lanosterol, a common and commercially available triterpenoid that is identical to hydroxy-8,24-dien-21-al apart from the aldehyde moiety at C21. 
In addition to exhibiting complement fixating activity, it was demonstrated that hydroxy-8,24dien-21-al and inotodiol showed activating rather than inhibiting activity on the complement cascade. This was shown by varying incubation times ( 0 and $30 \mathrm{~min})$ of the compounds with complement-containing serum before attempting to lyse blood cells. An inhibition of complement proteins would cause similar $\mathrm{ICH}_{50}$ values for both time points, which was the case for the melanin pigment and for the positive control heparin, which is a known complement inhibitor. An activation of complement proteins would cause depletion of activity over time due to the cascade being "used up" before reaching the blood cells they are supposed to lyse (118). For hydroxy-8,24-dien-21-al and inotodiol, the $\mathrm{ICH}_{50}$ values were significantly higher at 0 min than at $30 \mathrm{~min}$. In fact, inotodiol was virtually inactive at $0 \mathrm{~min}$ incubation, whereas hydroxy8,24-dien-21-al had an $\mathrm{ICH}_{50}$ value of $33 \pm 5.1 \mu \mathrm{M}$ at 0 min incubation vs $1.8 \pm 0.4 \mu \mathrm{M}$ at 30 min incubation. By using a commercially available ELISA kit, it was further demonstrated that the two triterpenoids acted through the classical and lectin pathways but not through the alternative pathway. According to the manufacturer of the kit (119) the activation of these two pathways simultaneously suggests that the complement cascade was induced through complement proteins $\mathrm{C} 2$ and/or $\mathrm{C} 4$, which are shared by the two pathways. Because complement activation might be beneficial in some disease states including cancer $(63,64)$, it would be interesting to follow up these results and possibly carry out in vivo experiments in order to investigate the potential of hydroxy-8,24-dien-21-al as a future complement-interacting drug.

In addition to the experiments related to the complement system, an anti-inflammatory cellbased experiment was conducted. This was carried out using the Griess assay, by activating bone marrow-derived macrophages with LPS and IFN- $\gamma$ to produce NO and then attempt to inhibit or reduce NO levels in the cell supernatants by treating the cells with triterpenoids. Among the triterpenoids, only betulin-3-O-caffeate displayed moderate activity in this experiment, with an $\mathrm{IC}_{50}$ value of $17.6 \pm 3.7 \mu \mathrm{M}$. The cytotoxic compounds betulinic acid, betulin and inotodiol first appeared to reduce NO levels as well, but an MTT assay carried out subsequent of the Griess assay demonstrated that this was due to cytotoxicity of the compounds. Interestingly, betulin-3-O-caffeate was completely non-toxic according to the MTT assays carried out on BMDMs and the cancer cell lines, whereas betulin was not. It therefore seems evident that the caffeic acid moiety rendered the compound non-toxic while giving it anti- 
inflammatory properties. It could be that the reduction in NO by betulin-3-O-caffeate was due to its antioxidant properties, but other triterpenoids with 3-O-caffeate moieties such as ursolic acid-3 $O$-caffeate were shown to not display NO reducing activity in similar experimental setups (120). Therefore, the anti-inflammatory activity of betulin-3-O-caffeate should be explored further, e.g. by quantifying cytokines such as TNF- $\alpha$ instead of NO.

Apart from the immunological activities, betulinic acid, betulin and inotodiol had an antiproliferative activity toward the lung carcinoma cell line H460 and the colorectal adenocarcinoma cell line HT29-MTX. It is well known from the literature that betulinic acid and inotodiol have cytotoxic activities against cancer cells $(43,46)$. However, to this author's knowledge these compounds have not been tested against the HT29-MTX cell line before. This cell line is resistant to the chemotherapeutic drug methotrexate (121), and have some different properties than its "mother" cell line HT29 due to the development of a resistance mechanism against methotrexate (122). The strong anti-proliferative effects of the isolated triterpenoids against HT29-MTX (e.g. betulinic acid had an $\mathrm{IC}_{50}$ value of $0.8 \pm 0.3 \mu \mathrm{M}$ ) demonstrated that these compounds acted through a different mechanism than methotrexate, and this is interesting in a pharmaceutical point of view, as combination therapy in cancer treatment is now commonly used in clinical practice (123). In addition, the results are particularly interesting because ethanol extracts from I. obliquus are sold on the marked, even in Norway. Although the triterpenoids isolated in this thesis came from DCM extracts, they are present in ethanol extracts as well (124). Due to the potent anti-proliferative activity of the compounds toward HT29-MTX cells, it might be that ethanol extracts can have anti-cancer effects in the colon due to these triterpenoids. Due to the low degree of uptake of triterpenoids from the GI tract (125), they would likely pass through the small intestine and as such, tumors being present in the colon would be exposed to the compounds. Therefore, it would be worthwhile to investigate the in vivo effects of ethanol extracts and isolated triterpenoids on the general colorectal health and against colon cancer in particular. The direct anti-cancer activity of I. obliquus triterpenoids is also interesting because these compounds could potentiate the indirect anti-tumorigenic activities of the isolated polysaccharides. Thus, the combined actions of these highly different classes of natural products could lead to a more potent anti-cancer activity. Such a combination therapy should be explored in more detail using in vivo cancer-treatment experiments where differences between single treatments and combinations of compounds are compared. 


\section{Challenges regarding structural characterization of melanin}

In addition to the polysaccharides and triterpenoids described in this thesis, a melanin fraction was also isolated and characterized. Although the characterization of melanin was not an initial aim of the thesis, it became evident quite early that the water-extracts contained massive amounts of dark pigment, later identified as melanin. In order to get a better picture of which of the I. obliquus compounds had biological activities, a thorough investigation of the melanin pigment was warranted. As demonstrated in the biological assays, the isolated melanin fraction had immunomodulating properties. The melanin fraction exhibited inhibitory effects on the complement cascade through all three pathways, with an $\mathrm{ICH}_{50}$ value $=8.4 \pm 0.1 \mu \mathrm{g} / \mathrm{mL}$ (after 0 min incubation). In addition, it showed moderate anti-inflammatory effects ( IC $_{50}$ value $=24.1$ $\pm 7.9 \mu \mathrm{g} / \mathrm{mL}$ ) by reducing NO levels in the macrophage supernatant after activation with LPS + IFN- $\gamma$.

After discovering the immunomodulating activity of the melanin pigment, it was attempted to characterize its structure. A common way of characterizing the chemical structure of melanin is to first separate it from other compounds by a prolonged treatment with highly acidic solvents (e.g. $3 \mathrm{M} \mathrm{HCl}$ ) (126), before analyzing the melanin pigment by spectroscopic or chromatographic methods. However, this harsh treatment renders the pigment insoluble except at high $\mathrm{pH}(>12)$, and thus characterization of its biological activities would become difficult. The water-soluble melanin fraction used in the biological experiments in this thesis was obtained by treating the crude water-soluble "polysaccharide extract" IOI-W several times with $0.1 \mathrm{M} \mathrm{HCl}$ in order to precipitate the pigment. After centrifugation of this solution, watersoluble polymers such as polysaccharides would be present in the supernatant, which could then be discarded. When the melanin precipitate was re-dissolved in neutral $(\mathrm{pH} 7)$ water and dialyzed, it was still water-soluble, and now likely to be free of other polymers or low-molecular weight compounds (see paper III for detailed isolation steps). It was attempted to analyze this fraction using NMR spectroscopy and MS, to get a clue of what type of monomeric unit the pigment consisted of. However, this was not successful, even after extensive testing and optimization of the experiments. Melanin pigments seem to be among the most difficult natural products to characterize, and the structure of fungal melanin pigments is still poorly understood (53). For example, NMR spectroscopy is often unsuitable for their characterization, although solid-state NMR might be useful to a certain extent (127). It was therefore attempted to characterize the pigment by other methods. First, it was revealed by GC analysis that the melanin fraction contained $\sim 5 \%$ carbohydrates, including Glc, Gal and GalA. Because these 
carbohydrates could not be removed by the precipitation technique, it was suggested that they were covalently bound to the melanin pigment. Further, using a SEC column and dextran standards, the approximate molecular weight of the polymer was estimated to be less than 10 $\mathrm{kDa}$. In addition to these methods, it was attempted to characterize the structure of the melanin polymer. To achieve this, the pigment was first treated with $6 \mathrm{M} \mathrm{HCl}$ for $24 \mathrm{~h}$ to remove all carbohydrates. Then, the pigment was degraded using $\mathrm{H}_{2} \mathrm{O}_{2}$ in alkaline conditions before analyzing the degradation products by reverse phase HPLC. The degradation products were compared with eumelanin degradation products from $S$. officinalis and with the known eumelanin markers pyrrole-2,3,5-tricarboxylic acid (PTCA) and pyrrole-2,3-dicarboxylic acid (PDCA) $(128,129)$. The degradation products from the I. obliquus melanin fraction did not match any of the markers, and it was concluded that the pigment consisted of different building blocks than the eumelanin from S. officinalis. It was therefore attempted to analyze the main degradation products of the I. obliquus melanin further by NMR spectroscopy and MS, but this attempt was not successful. One important reason was that the phosphate buffer used for HPLC analysis seemed to interfere with the isolation, separation and characterization of the markers when using preparative HPLC, but this buffer was required for sufficient separation and solubility of the degradation product in the first place. It should be attempted to optimize this procedure further, because the structure of the degradation products could give important clues on the overall structure of the melanin pigments (128). Further, combustion analysis revealed the I. obliquus pigment to be devoid of nitrogen, in contrast to the eumelanin from S. officinalis. Therefore, it was likely that the I. obliquus pigment was of the allomelanin/DHN type, which is one of the most common types of melanin in fungi and also one of the only nitrogen-devoid types of melanin (52). However, due to the insufficient characterization of the pigment, it should be attempted to investigate its structure further. One way of doing this is by growing mycelium in culture, and then block different known melanin-producing pathways, as this is a common way of determining the building blocks of the melanin pigments.

\section{Using cell-based in vitro assays as a model system}

Cell-based in vitro assays are a powerful tool to investigate biological effects of natural products. However, such assays have many limitations. First, an obvious question is how the results from in vitro experiments translate to the in vivo situation. Bioavailability of the compounds is not an issue in vitro, but is usually a key parameter to consider for in vivo 
experiments. The bioavailability of a compound is dependent on many factors, such as molecular weight, charge, solubility and chemical and biological stability. Another important feature that is lost in vitro is the dynamic environment that cells live in in vivo. As discussed earlier, macrophages are highly dynamic cells that constantly adapt to their surroundings, and therefore it is unlikely that cultured macrophages will have the same phenotype as they have in vivo, even when primary bone marrow-derived macrophages are used. As a notable example, there is a discrepancy when it comes to NO production by human macrophages in vitro and in vivo. In vitro, the iNOS gene responsible for NO production appears to be switched off (at least under certain conditions), but human macrophages are known to produce NO in vivo (130, 131). This demonstrates that in vitro experiments should be followed up using in vivo experiments if a given compound has interesting activities, to assess the validity of the results.

When performing biological experiments, the logical way of thinking is that compounds are more active in vitro than in vivo, but this is not always the case. For example, the growth inhibition assay presented in this thesis was carried out in vivo as well by researchers in our group. In this experiment, $\mathrm{Pam}_{3} \mathrm{CSK}_{4}+\mathrm{IFN}-\gamma$ were used to activate macrophages, before the macrophages were injected sub-cutaneously in mice along with cancer cells. The results clearly showed that the activated macrophages gave an eradication of the tumor (unpublished results) in the mice, as opposed to the control group that was injected with untreated macrophages. When the mice were re-challenged with cancer cells on the opposite side of the body some weeks later, the mice that had received treatment with activated macrophages did not develop a tumor, indicating a protective, systemic effect by the original treatment. This effect would not have been possible in vitro and further underlines the importance of following up in vitro experiments with similar experiments in vivo. 


\section{CONCLUDING REMARKS AND FUTURE PERSPECTIVES}

The overall aim of this thesis was to isolate and chemically characterize compounds from $I$. obliquus and to investigate the immunomodulating properties of these compounds.

Complex and heterogeneous polysaccharides were isolated from the I. obliquus water extracts, and these induced pro-inflammatory and anti-tumorigenic effects in murine macrophages, likely through the activation of TLR2, TLR4 and Dectin-1a.

Further, six triterpenoids were isolated from the I. obliquus dichloromethane extracts. $3 \beta$ hydroxy-8,24-dien-21-al and inotodiol had potent activating effects on the classical and lectin complement pathways, whereas betulin-3-O-caffeate displayed anti-inflammatory activity by reducing nitric oxide concentration subsequent of macrophages activation. In addition, betulinic acid, betulin and inotodiol exhibited potent anti-proliferative activities against the cancer cell lines HT29-MTX and NCI-H460.

Finally, a DHN-type melanin pigment was isolated from an I. obliquus water extract, and was shown to have potent inhibitory effects on the classical, lectin and alternative complement pathways, as well as an anti-inflammatory activity by reducing nitric oxide concentration subsequent of macrophage activation.

Some of the results presented in this thesis warrant further investigation. The macrophage activation by the polysaccharides should be explored using in vivo tumor growth inhibition experiments, and the in vitro experiments should be repeated using human macrophages. Furthermore, interaction with other receptors apart from TLR2, TLR4 and Dectin-1a, such as CR3, should be investigated. It should also be attempted to use enzymes to partially degrade the polysaccharides in order to analyze their structure further and to potentially increase their activity.

The complement-activating effect of $3 \beta$-hydroxy-8,24-dien-21-al should be investigated further, for example by using a library of triterpenoids with similar chemical composition to identify the pharmacophore(s) of the compound. Additionally, the exact mechanism of action of the compound should be identified, and in vivo experiments should be used to verify its biological activity further.

The structure of the melanin pigment should be analyzed in more detail, by analyzing degradation products after treatment with $\mathrm{H}_{2} \mathrm{O}_{2}$ and possibly by solid-state NMR. In addition, 
in vivo experiments should be conducted to investigate the bioavailability of the pigment and its effects on the gastrointestinal system.

Collectively, the results presented in this thesis demonstrate the immunomodulating activities of polysaccharides, triterpenoids and melanin from I. obliquus. Interestingly, the different classes of compounds showed either pro- or anti-inflammatory effects in vitro. Therefore, it can be concluded that I. obliquus contains compounds that are able to elicit different types of immune responses, through either activation or inhibition of macrophages and the complement cascade. 


\section{REFERENCES}

1. Newman DJ, Cragg GM. Natural products as sources of new drugs from 1981 to 2014. J Nat Prod. 2016;79(3):629-61.

2. Gardens KRB. State of the world's fungi 2018 [Available from:

https://stateoftheworldsfungi.org/2018/reports/SOTWFungi 2018 Full Report.pdf, accessed 11.06.2020.

3. Hawksworth DL, Lucking R. Fungal diversity revisited: 2.2 to 3.8 million species. Microbiol Spectr. 2017;5(4).

4. de Mattos-Shipley KM, Ford KL, Alberti F, Banks AM, Bailey AM, Foster GD. The good, the bad and the tasty: The many roles of mushrooms. Stud Mycol. 2016;85:125-57.

5. Jin X, Ruiz Beguerie J, Sze DM, Chan GC. Ganoderma lucidum (Reishi mushroom) for cancer treatment. Cochrane Database Syst Rev. 2016;4:CD007731.

6. Zhang $M$, Zhang $Y$, Zhang $L$, Tian $Q$. Mushroom polysaccharide lentinan for treating different types of cancers: A review of 12 years clinical studies in China. Prog Mol Biol Transl Sci. 2019;163:297-328.

7. Hetland G, Johnson E, Lyberg T, Kvalheim G. The mushroom Agaricus blazei Murill elicits medicinal effects on tumor, infection, allergy, and inflammation through its modulation of innate immunity and amelioration of Th1/Th2 imbalance and inflammation. Adv Pharmacol Sci. 2011;2011:157015.

8. Min-Woong L, Hyeon-Hur, Kwang-Choon C, Tae-Soo L, Kang-Hyeon K, Jankovsky L. Introduction to distribution and ecology of sterile conks of Inonotus obliquus. Mycobiology. 2008;36(4):199-202.

9. Cha JY, Lee SY, Lee SY, Chun KW. Basidiocarp formation by Inonotus obliquus on a living paper birch tree. For Pathol. 2011;41(2):163-4.

10. Saar M. Fungi in Khanty folk medicine. J Ethnopharmacol. 1991:175-9.

11. Shikov AN, Pozharitskaya ON, Makarov VG, Wagner H, Verpoorte R, Heinrich M. Medicinal plants of the Russian Pharmacopoeia; their history and applications. J Ethnopharmacol. 2014;154(3):481-536.

12. EuropeanCommission. EU Novel food catalogue (v.1.1), Inonotus obliquus [Available from: https://ec.europa.eu/food/safety/novel food/catalogue/search/public/?event=home\&seqfce=421\& ascii=I\#, accessed 07.05.2020.

13. Spinosa R. The Chaga story, The Mycophile (by the North American mycological association)

2006. Available from: https://namyco.org/docs/MycoJanFeb06.pdf , accessed 07.05.2020.

14. Arata S, Watanabe J, Maeda M, Yamamoto M, Matsuhashi $H$, Mochizuki M, et al. Continuous intake of the Chaga mushroom (Inonotus obliquus) aqueous extract suppresses cancer progression and maintains body temperature in mice. Heliyon. 2016;2(5):e00111.

15. Chung MJ, Chung CK, Jeong Y, Ham SS. Anticancer activity of subfractions containing pure compounds of Chaga mushroom (Inonotus obliquus) extract in human cancer cells and in Balbc/c mice bearing Sarcoma-180 cells. Nutr Res Pract. 2010;4(3):177-82.

16. Chen Y, Huang Y, Cui Z, Liu J. Purification, characterization and biological activity of a novel polysaccharide from Inonotus obliquus. Int J Biol Macromol. 2015;79:587-94.

17. Kim YO, Park HW, Kim JH, Lee JY, Moon SH, Shin CS. Anti-cancer effect and structural characterization of endo-polysaccharide from cultivated mycelia of Inonotus obliquus. Life Sci. 2006;79(1):72-80.

18. Gery A, Dubreule C, Andre V, Rioult JP, Bouchart V, Heutte N, et al. Chaga (Inonotus obliquus), a future potential medicinal fungus in oncology? A chemical study and a comparison of the cytotoxicity against human lung adenocarcinoma cells (A549) and human bronchial epithelial cells (BEAS-2B). Integr Cancer Ther. 2018;17(3):832-43. 
19. Kukulyanskaya TA, Kurchenko NV, Kurchenko VP, Babitskaya VG. Physicochemical properties of melanins produced by the sterile form of Inonotus obliquus ("Chagi") in natural and cultivated fungus. Appl Biochem Microbiol. 2002;38(1):58-61.

20. Wold CW, Kjeldsen C, Corthay A, Rise F, Christensen BE, Duus JO, et al. Structural characterization of bioactive heteropolysaccharides from the medicinal fungus Inonotus obliquus (Chaga). Carbohydr Polym. 2018;185:27-40.

21. Hwang BS, Lee IK, Yun BS. Phenolic compounds from the fungus Inonotus obliquus and their antioxidant properties. J Antibiot (Tokyo). 2016;69(2):108-10.

22. Daley DK, Brown KJ, Badal S. Fungal metabolites. Pharmacognosy2017. p. 413-21.

23. Erwig LP, Gow NA. Interactions of fungal pathogens with phagocytes. Nat Rev Microbiol. 2016;14(3):163-76.

24. Liu J, Willför S, Xu C. A review of bioactive plant polysaccharides: Biological activities, functionalization, and biomedical applications. Bioact Carbohydr Diet Fibre. 2015;5(1):31-61.

25. Parada Venegas D, De la Fuente MK, Landskron G, Gonzalez MJ, Quera R, Dijkstra G, et al. Short chain fatty acids (SCFAs)-mediated gut epithelial and immune regulation and its relevance for inflammatory bowel disease. Front Immunol. 2019;10:277.

26. Canfora EE, Jocken JW, Blaak EE. Short-chain fatty acids in control of body weight and insulin sensitivity. Nat Rev Endocrinol. 2015;11(10):577-91.

27. Batbayar S, Lee DH, Kim HW. Immunomodulation of fungal beta-glucan in host defense signaling by Dectin-1. Biomol Ther (Seoul). 2012;20(5):433-45.

28. Synytsya A, Novak M. Structural diversity of fungal glucans. Carbohydr Polym. 2013;92(1):792-809.

29. Goodridge HS, Andrea J. Wolf AJ, Underhill DM. beta-glucan recognition by the innate immune system. Immunol Rev. 2009;230:38-50.

30. Rieder A, Samuelsen AB. Do cereal mixed-linked beta-glucans possess immune-modulating activities? Mol Nutr Food Res. 2012;56(4):536-47.

31. Whitehead A, Beck EJ, Tosh S, Wolever TM. Cholesterol-lowering effects of oat beta-glucan: a meta-analysis of randomized controlled trials. Am J Clin Nutr. 2014;100(6):1413-21.

32. Sasaki T, Takasuka N. Further study of the structure of Lentinan, an anti-tumor polysaccharide from Lentinus edodes. Carbohydr Res. 1976;47:99-104.

33. Strahl-Bolsinger S, Gentzsch M, Tanner W. Protein O-mannosylation. Biochim Biophys Acta. 1999;1426:297-307.

34. Taylor PR, Tsoni SV, Willment JA, Dennehy KM, Rosas M, Findon $\mathrm{H}$, et al. Dectin-1 is required for beta-glucan recognition and control of fungal infection. Nat Immunol. 2007;8(1):31-8.

35. Huang SQ, Ding S, Fan L. Antioxidant activities of five polysaccharides from Inonotus obliquus. Int J Biol Macromol. 2012;50(5):1183-7.

36. Quin MB, Flynn CM, Schmidt-Dannert C. Traversing the fungal terpenome. Nat Prod Rep. 2014;31(10):1449-73.

37. Hillier SG, Lathe R. Terpenes, hormones and life: isoprene rule revisited. J Endocrinol. 2019;242(2):R9-R22.

38. Jia Q, Chen X, Kollner TG, Rinkel J, Fu J, Labbe J, et al. Terpene synthase genes originated from bacteria through horizontal gene transfer contribute to terpenoid diversity in fungi. Sci Rep. 2019;9(1):9223.

39. Xia $Q$, Zhang $H$, Sun $X, Z$ hao $H$, Wu L, Zhu D, et al. A comprehensive review of the structure elucidation and biological activity of triterpenoids from Ganoderma spp. Molecules. 2014;19(11):17478-535.

40. Liu C, Zhao C, Pan HH, Kang J, Yu XT, Wang HQ, et al. Chemical constituents from Inonotus obliquus and their biological activities. J Nat Prod. 2014;77(1):35-41.

41. Zheng W, Miao K, Liu Y, Zhao Y, Zhang M, Pan S, et al. Chemical diversity of biologically active metabolites in the sclerotia of Inonotus obliquus and submerged culture strategies for up-regulating their production. Appl Microbiol Biotechnol. 2010;87(4):1237-54. 
42. Zhang SD, Yu L, Wang P, Kou P, Li J, Wang LT, et al. Inotodiol inhibits cells migration and invasion and induces apoptosis via p53-dependent pathway in HeLa cells. Phytomedicine. 2019;60:152957.

43. Nomura M, Takahashi T, Uesugi A, Tanaka R, Kobayashi S. Inotodiol, a lanostane triterpenoid, from Inonotus obliquus inhibits cell proliferation through caspase-3-dependent apoptosis. Anticancer Res. 2008;28:2691-6.

44. Scheffler A. The wound healing properties of betulin from birch bark: from bench to bedside. Planta Med. 2019;85(7):524-7.

45. Pisha E, Chai H, Lee I-S, Chagwedera TE, Farnsworth NR, Cordell GA, et al. Discovery of betulinic acid as a selective inhibitor of human melanoma that functions by induction of apoptosis. Nature Medicine. 1995;1(1046-1051).

46. Zuco V, Supino R, Righetti SC, Cleris L, Marchesi E, Gambacorti-Passerini C, et al. Selective cytotoxicity of betulinic acid on tumor cell lines, but not on normal cells. Cancer Letters. 2002;175:17-25.

47. Kvasnica M, Sarek J, Klinotova E, Dzubak P, Hajduch M. Synthesis of phthalates of betulinic acid and betulin with cytotoxic activity. Bioorg Med Chemi. 2005;13(10):3447-54.

48. Nguyen TMN, Le HS, Le BV, Kim YH, Hwang I. Anti-allergic effect of inotodiol, a lanostane triterpenoid from Chaga mushroom, via selective inhibition of mast cell function. Int Immunopharmacol. 2020;81:106244.

49. Smith PF, Ogundele A, Forrest A, Wilton J, Salzwedel K, Doto J, et al. Phase I and II study of the safety, virologic effect, and pharmacokinetics/pharmacodynamics of single-dose 3-o-(3',3'dimethylsuccinyl)betulinic acid (bevirimat) against human immunodeficiency virus infection. Antimicrob Agents Chemother. 2007;51(10):3574-81.

50. Cordero RJ, Casadevall A. Functions of fungal melanin beyond virulence. Fungal Biol Rev. 2017;31(2):99-112.

51. Greco G, Panzella L, Verotta L, d'Ischia M, Napolitano A. Uncovering the structure of human red hair pheomelanin: benzothiazolylthiazinodihydroisoquinolines as key building blocks. J Nat Prod. 2011;74(4):675-82.

52. Eisenman HC, Casadevall A. Synthesis and assembly of fungal melanin. Appl Microbiol Biotechnol. 2012;93(3):931-40.

53. Nosanchuk JD, Stark RE, Casadevall A. Fungal melanin: What do we know about structure? Front Microbiol. 2015;6:1463.

54. Stappers MHT, Clark AE, Aimanianda V, Bidula S, Reid DM, Asamaphan P, et al. Recognition of DHN-melanin by a C-type lectin receptor is required for immunity to Aspergillus. Nature. 2018;555(7696):382-6.

55. Rosas AL, MacGill RS, Nosanchuk JD, Kozel TR, Casadevall A. Activation of the alternative complement pathway by fungal melanins. Clin Diagn Lab Immunol. 2002;9(1):144-8.

56. Kukulyanskaya TA, Kurchenko NV, Kurchenko VP, Babitskaya VG. Physicochemical properties of melanins produced by the sterile form of Inonotus obliquus ("Chagi") in natural and cultivated fungus. Applied Biochemistry and Microbiology. 2002;38(1):58-61.

57. Chaplin DD. Overview of the immune response. J Allergy Clin Immunol. 2010;125(2 Suppl 2):S3-23.

58. Ricklin D, Hajishengallis G, Yang K, Lambris JD. Complement: a key system for immune surveillance and homeostasis. Nat Immunol. 2010;11(9):785-97.

59. Zhang $Y$, Zhang $M$, Jiang $Y$, Li $X, H e ~ Y$, Zeng $P$, et al. Lentinan as an immunotherapeutic for treating lung cancer: a review of 12 years clinical studies in China. J Cancer Res Clin Oncol. 2018;144(11):2177-86.

60. Cohen DJ, Loertscher R, Rubin MF, Tilney NL, Carpenter CB, Strom TB. Cyclosporine - A new immunosuppressive agent for organ transplantation. Ann Internal Med. 1984;101:667-82.

61. Xu J, Yi M, Ding L, He S. A review of anti-inflammatory compounds from marine fungi, 20002018. Mar Drugs. 2019;17(11). 
62. Ricklin D, Reis ES, Lambris JD. Complement in disease: a defence system turning offensive. Nat Rev Nephrol. 2016;12(7):383-401.

63. Mamidi S, Hone S, Kirschfink M. The complement system in cancer: Ambivalence between tumour destruction and promotion. Immunobiology. 2017;222(1):45-54.

64. Macor P, Capolla S, Tedesco F. Complement as a biological tool to control tumor growth. Front Immunol. 2018;9:2203.

65. Forman $\mathrm{HJ}$, Torres $\mathrm{M}$. Reactive oxygen species and cell signaling: respiratory burst in macrophage signaling. Am J Respir Crit Care Med. 2002;166(12 Pt 2):S4-8.

66. Pathria P, Louis TL, Varner JA. Targeting tumor-associated macrophages in cancer. Trends Immunol. 2019;40(4):310-27.

67. Morrison C. Industry shows increased appetite for macrophage biology: Nat. Rev. Drug

Discov.; 2020 [Available from: https://www.nature.com/articles/d41573-020-000753?fbclid=IwAR18AayqeCPk4sX8SQOsxj7FB8rA0voURvfSP01-YJ2jbyB59RBOHRuCyp8, accessed 11.06.2020.

68. Lundahl MLE, Scanlan EM, Lavelle EC. Therapeutic potential of carbohydrates as regulators of macrophage activation. Biochem Pharmacol. 2017;146:23-41.

69. Thorsson V, Gibbs DL, Brown SD, Wolf D, Bortone DS, Ou Yang TH, et al. The immune landscape of cancer. Immunity. 2018;48(4):812-30 e14.

70. Gajewski TF, Schreiber H, Fu YX. Innate and adaptive immune cells in the tumor microenvironment. Nat Immunol. 2013;14(10):1014-22.

71. Takeya $\mathrm{M}$, Komohara $\mathrm{Y}$. Role of tumor-associated macrophages in human malignancies: friend or foe? Pathol Int. 2016;66(9):491-505.

72. Riabov V, Gudima A, Wang N, Mickley A, Orekhov A, Kzhyshkowska J. Role of tumor associated macrophages in tumor angiogenesis and lymphangiogenesis. Front Physiol. 2014;5:75.

73. Mills CD, Lenz LL, Harris RA. A breakthrough: Macrophage-directed cancer immunotherapy. Cancer Res. 2016;76(3):513-6.

74. Mills CD. Anatomy of a discovery: $m 1$ and $m 2$ macrophages. Front Immunol. 2015;6:212.

75. Xue J, Schmidt SV, Sander J, Draffehn A, Krebs W, Quester I, et al. Transcriptome-based network analysis reveals a spectrum model of human macrophage activation. Immunity. 2014;40(2):274-88.

76. Muller E, Christopoulos PF, Halder S, Lunde A, Beraki K, Speth M, et al. Toll-like receptor ligands and interferon-gamma synergize for induction of antitumor M1 macrophages. Front Immunol. 2017;8:1383.

77. Corthay A, Skovseth DK, Lundin KU, Rosjo E, Omholt H, Hofgaard PO, et al. Primary antitumor immune response mediated by CD4+ T cells. Immunity. 2005;22(3):371-83.

78. Hammerich L, Marron TU, Upadhyay R, Svensson-Arvelund J, Dhainaut M, Hussein S, et al. Systemic clinical tumor regressions and potentiation of PD1 blockade with in situ vaccination. Nat Med. 2019;25(5):814-24.

79. Kumar H, Kawai T, Akira S. Pathogen recognition by the innate immune system. Int Rev Immunol. 2011;30(1):16-34.

80. Fitzgerald KA, Kagan JC. Toll-like receptors and the control of immunity. Cell. 2020.

81. Park BS, Lee JO. Recognition of lipopolysaccharide pattern by TLR4 complexes. Exp Mol Med. 2013;45:e66.

82. Oliveira-Nascimento L, Massari P, Wetzler LM. The role of TLR2 in infection and immunity. Front Immunol. 2012;3:79.

83. Bourgeois C, Kuchler K. Fungal pathogens-a sweet and sour treat for toll-like receptors. Front Cell Infect Microbiol. 2012;2:142.

84. Dambuza IM, Brown GD. C-type lectins in immunity: recent developments. Curr Opin Immunol. 2015;32:21-7.

85. Kerrigan AM, Brown GD. Syk-coupled C-type lectins in immunity. Trends Immunol. 2011;32(4):151-6. 
86. Brown GD, Taylor PR, Reid DM, Willment JA, Williams DL, Martinez-Pomares L, et al. Dectin-1 is a major beta-glucan receptor on macrophages. J Exp Med. 2002;196(3):407-12.

87. Goodridge HS, Reyes CN, Becker CA, Katsumoto TR, Ma J, Wolf AJ, et al. Activation of the innate immune receptor Dectin-1 upon formation of a 'phagocytic synapse'. Nature. 2011;472(7344):471-5.

88. Ferwerda G, Meyer-Wentrup F, Kullberg BJ, Netea MG, Adema GJ. Dectin-1 synergizes with TLR2 and TLR4 for cytokine production in human primary monocytes and macrophages. Cell Microbiol. 2008;10(10):2058-66.

89. Yadav M, Schorey JS. The beta-glucan receptor dectin-1 functions together with TLR2 to mediate macrophage activation by mycobacteria. Blood. 2006;108(9):3168-75.

90. Khan T, Date A, Chawda H, Patel K. Polysaccharides as potential anticancer agents - A review of their progress. Carbohydr Polym. 2019;210:412-28.

91. Bao X, Yuan H, Wang C, Liu J, Lan M. Antitumor and immunomodulatory activities of a polysaccharide from Artemisia argyi. Carbohydr Polym. 2013;98(1):1236-43.

92. Fritz H, Kennedy DA, Ishii M, Fergusson D, Fernandes R, Cooley K, et al. Polysaccharide K and Coriolus versicolor extracts for lung cancer: a systematic review. Integr Cancer Ther. 2015;14(3):20111.

93. Huerta S. Nitric oxide for cancer therapy. Future Sci OA. 2015;1(1):FSO44.

94. Appleton DR, Buss AD, Butler MS. A simple method for high-throughput extract pre-

fractionation for biological screening. CHIMIA. 2007;61(6):327-31.

95. Carbonero ER, Gracher AH, Rosa MC, Torri G, Sassaki GL, Gorin PA, et al. Unusual partially 3O-methylated alpha-galactan from mushrooms of the genus Pleurotus. Phytochemistry. 2008;69(1):252-7.

96. Yang Y, Zhang J, Liu Y, Tang Q, Zhao Z, Xia W. Structural elucidation of a 3-O-methyl-Dgalactose-containing neutral polysaccharide from the fruiting bodies of Phellinus igniarius. Carbohydr Res. 2007;342(8):1063-70.

97. Staudacher E. Methylation - an uncommon modification of glycans. J Biol Chem. 2012;393(8):675-85.

98. Muschin T, Yoshida T. Structural analysis of galactomannans by NMR spectroscopy. Carbohydr Polym. 2012;87(3):1893-8.

99. Free SJ. Fungal cell wall organization and biosynthesis. Adv Genet. 2013;81:33-82.

100. Inngjerdingen KT, Patel TR, Chen X, Kenne L, Allen S, Morris GA, et al. Immunological and structural properties of a pectic polymer from Glinus oppositifolius. Glycobiology. 2007;17(12):1299310.

101. Berlemont R. Distribution and diversity of enzymes for polysaccharide degradation in fungi. Sci Rep. 2017;7(1):222.

102. Narui T, Iwata S, Takahashi K, Shibata S. Partial hydrolysis of alpha-D-glucans with acid in the presence of 1,1,3,3-tetramethylurea. Carbohydr Res. 1987;170:269-73.

103. Vogt LM, Sahasrabudhe NM, Ramasamy U, Meyer D, Pullens G, Faas MM, et al. The impact of lemon pectin characteristics on TLR activation and T84 intestinal epithelial cell barrier function. J Funct Foods. 2016;22:398-407.

104. Sahasrabudhe NM, Beukema M, Tian L, Troost B, Scholte J, Bruininx E, et al. Dietary fiber pectin directly blocks toll-like receptor 2-1 and prevents doxorubicin-induced ileitis. Front Immunol. 2018;9.

105. Camilli G, Tabouret G, Quintin J. The complexity of fungal beta-glucan in health and disease: effects on the mononuclear phagocyte system. Front Immunol. 2018;9:673.

106. Vetvicka V, Thornton BP, Ross GD. Soluble beta-glucan polysaccharide binding to the lectin site of neutrophil or natural killer cell complement receptor type 3 (CD11b/CD18) generates a primed state of the receptor capable of mediating cytotoxicity of iC3b-opsonized target cells. J Clin Invest. 1996;98(1). 
107. Zhang J, Tyler HL, Haron MH, Jackson CR, Pasco DS, Pugh ND. Macrophage activation by edible mushrooms is due to the collaborative interaction of toll-like receptor agonists and dectin- $1 \mathrm{~b}$ activating beta glucans derived from colonizing microorganisms. Food Funct. 2019;10(12):8208-17. 108. Wold CW, Gerwick WH, Wangensteen H, Inngjerdingen KT. Bioactive triterpenoids and water-soluble melanin from Inonotus obliquus (Chaga) with immunomodulatory activity. J Funct Foods. 2020;71.

109. Lieder R, Petersen PH, Sigurjonsson OE. Endotoxins-the invisible companion in biomaterials research. Tissue Eng Part B Rev. 2013;19(5):391-402.

110. Gaikwad S, Agrawal-Rajput R. Lipopolysaccharide from Rhodobacter sphaeroides attenuates microglia-mediated inflammation and phagocytosis and directs regulatory $T$ cell response. Int J Inflam. 2015;2015:361326.

111. Pugh ND, Tamta H, Balachandran P, Wu X, Howell J, Dayan FE, et al. The majority of in vitro macrophage activation exhibited by extracts of some immune enhancing botanicals is due to bacterial lipoproteins and lipopolysaccharides. Int Immunopharmacol. 2008;8(7):1023-32.

112. Chapter 6 The chemical characterisation of lipoproteins. A Guidebook to Lipoprotein Technique. Laboratory Techniques in Biochemistry and Molecular Biology1984. p. 221-307.

113. Kahlos K, Hiltunen R, Schantz Mv. 33-Hydroxy-lanosta-8,24-dien-21-al, a new triterpene from Inontus obliquus. Planta Med. 1984;50(2):197-8.

114. van Asbeck EC, Hoepelman Al, Scharringa J, Herpers BL, Verhoef J. Mannose binding lectin plays a crucial role in innate immunity against yeast by enhanced complement activation and enhanced uptake of polymorphonuclear cells. BMC Microbiol. 2008;8:229.

115. Ho GT, Wangensteen $\mathrm{H}$, Barsett $\mathrm{H}$. Elderberry and elderflower extracts, phenolic compounds, and metabolites and their effect on complement, RAW 264.7 macrophages and dendritic Cells. Int J Mol Sci. 2017;18(3).

116. Rauf A, Imran M, Abu-Izneid T, lahtisham UI H, Patel S, Pan X, et al. Proanthocyanidins: A comprehensive review. Biomed Pharmacother. 2019;116:108999.

117. Seo HW, Hung TM, Na M, Jung HJ, Kim JC, Choi JS, et al. Steroids and triterpenes from the fruit bodies of Ganoderma lucidum and their anti-complement activity. Arch Pharm Res. 2009;32(11):1573-9.

118. Alban S, Classen B, Brunner G, Blaschek W. Differentiation between the complement modulating effects of an Arabinogalactan-protein from Echinacea purpurea and heparin. Planta Med. 2002;68:1118-24.

119. SvarLifeScience. WIESLAB ${ }^{\circledR}$ Complement system screen 2015 [Available from: https://cdn2.hubspot.net/hubfs/2441243/Product\%20Documents\%20/COMPL300\%20\%20COMPL300RUO/COMPL\%20300\%20RUO\%20LABEL-DOC-0028\%20v2\%200.pdf (accessed March 13th 2020).

120. Jeong W, Hong SS, Kim N, Yang YT, Shin YS, Lee C, et al. Bioactive triterpenoids from Callistemon lanceolatus. Arch Pharm Res. 2009;32(6):845-9.

121. Lesuffleur T, Porchet N, Aubert J-P, Swallow D, Gum JR, Kim YS, et al. Differential expression of the human mucin genes MUC1 to MUC5 in relation to growth and differentiation of different mucus-secreting HT-29 cell subpopulations. J Cell Sci. 1993;106:771-83.

122. Bertino JR, Göker E, Gorlick R, Li WW, Banerjee D. Resistance mechanisms to methotrexate in tumors. Stem Cells. 1998;14:5-9.

123. Bozic I, Reiter JG, Allen B, Antal T, Chatterjee K, Shah P, et al. Evolutionary dynamics of cancer in response to targeted combination therapy. Elife. 2013;2:e00747.

124. Zhao F, Mai Q, Ma J, Xu M, Wang X, Cui T, et al. Triterpenoids from Inonotus obliquus and their antitumor activities. Fitoterapia. 2015;101:34-40.

125. NA JCF, Pirson L, Edelberg H, L MM, Loira-Pastoriza C, Preat V, et al. Pentacyclic triterpene bioavailability: An overview of in vitro and in vivo studies. Molecules. 2017;22(3).

126. Magarelli M, Passamonti P, Renieri C. Purification, characterization and analysis of sepia melanin from commercial sepia ink (Sepia officinalis). Ces Med Vet Zootec. 2010;5(2):18-28. 
127. Zhong J, Frases S, Wang H, Casadevall A, Stark RE. Following fungal melanin biosynthesis with solid-state NMR: biopolymer molecular structures and possible connections to cell-wall polysaccharides. Biochemistry. 2008;47:4701-10.

128. Ito S, Nakanishi Y, Valenzuela RK, Brilliant MH, Kolbe L, Wakamatsu K. Usefulness of alkaline hydrogen peroxide oxidation to analyze eumelanin and pheomelanin in various tissue samples: application to chemical analysis of human hair melanins. Pigment Cell Melanoma Res. 2011;24(4):605-13.

129. Ito S, Wakamatsu K, Ozeki H. Spectrophotometric assay of eumelanin in tissue samples. Anal Biochem. 1993;215(2):273-7.

130. Fang FC, Vazquez-Torres A. Nitric oxide production by human macrophages: there's NO doubt about it. Am J Physiol Lung Cell Mol Physiol. 2002;282:L941-L3.

131. Moncada S, Palmer RMJ, Higgs EA. Nitric oxide: Physiology, pathophysiology, and pharmacology. Pharmacol Rev. 1991;43(2):109-42. 
SCIENTIFIC PAPERS

\section{Paper I}

Structural characterization of bioactive heteropolysaccharides from the medicinal fungus Inonotus obliquus (Chaga)

Christian Winther Wold, Christian Kjeldsen, Alexandre Corthay, Frode Rise, Bjørn E. Christensen, Jens Øllgaard Duus, Kari Tvete Inngjerdingen

Carbohydrate Polymers (2018), 185: 27-40

\section{Paper II}

Polysaccharides from the fungus Inonotus obliquus (Chaga) activate macrophages into a tumoricidal phenotype via interaction with TLR2, TLR4 and Dectin-1a

Christian Winther Wold, Panagiotis Christopoulos, Inger Øynebråten, Maykel Arias, Alexandre Corthay, Kari Tvete Inngjerdingen

Manuscript

\section{Paper III}

Bioactive triterpenoids and water-soluble melanin from Inonotus obliquus (Chaga) with immunomodulatory activity

Christian Winther Wold, William H. Gerwick, Helle Wangensteen, Kari Tvete Inngjerdingen Journal of Functional Foods (2020), 71: 104025 

I 



\title{
Structural characterization of bioactive heteropolysaccharides from the medicinal fungus Inonotus obliquus (Chaga)
}

\author{
Christian Winther Wold ${ }^{\mathrm{a}, *}$, Christian Kjeldsen ${ }^{\mathrm{b}}$, Alexandre Corthay ${ }^{\mathrm{c}}$, Frode Rise ${ }^{\mathrm{e}}$, \\ Bjørn E. Christensen ${ }^{\mathrm{d}}$, Jens Øllgaard Duus ${ }^{\mathrm{b}}$, Kari Tvete Inngjerdingen ${ }^{\mathrm{a}}$ \\ a School of Pharmacy, University of Oslo, P.O. Box 1068 Blindern, N-0316 Oslo, Norway \\ b Department of Chemistry, Technical University of Denmark, DK-2800 Kgs. Lyngby, Denmark \\ ' Tumor Immunology Lab, Department of Pathology, Rikshospitalet, Oslo University Hospital, P.O. Box 4950 Nydalen, NO-0424 Oslo,Norway \\ ${ }^{\mathrm{d}}$ NOBIPOL, Department of Biotechnology and Food Science, Norwegian University of Science and Technology, N-7491 Trondheim, Norway \\ e Department of Chemistry, University of Oslo, P.O. Box 1033 Blindern, N-0315 Oslo, Norway
}

\section{A R T I C L E I N F O}

\section{Keywords:}

Chaga

Fungi

Polysaccharides

Structural characterization

Galactoglucan

NMR

\begin{abstract}
A B S T R A C T
The aim of this paper was to perform a comprehensive characterization of polysaccharides isolated from the interior (IOI) and exterior (IOE) parts of the fungus Inonotus obliquus. Pre-extraction with DCM and $\mathrm{MeOH}$ followed by water and alkali extraction and ethanol precipitation gave two water extracts and two alkali extracts. Neutral and acidic polysaccharide fractions were obtained after anion-exchange chromatography of the water extracts. The neutral polysaccharides $(60-73 \mathrm{kDa})$ were heterogeneous and branched and consisted of a $(1 \rightarrow 3)$-linked $\beta$-Glc backbone with $(1 \rightarrow 6)$-linked kinks in the chain at approximately every fifth residue, with branches of $(1 \rightarrow 6)$-linked $\beta$-Glc in addition to substantial amounts of $(1 \rightarrow 6)$-linked $\alpha$-Gal with 3-O-methylation at about every third Gal residue. The acidic polysaccharide fractions (10-31 kDa) showed similar structural motifs as the neutral fractions differing mainly by the presence of $(1 \rightarrow 4)$-linked $\alpha$-GalA and $\alpha$-GlcA. $\beta$-Xyl, $\alpha$ Man and $\alpha$-Rha were also present in varying amounts in all fractions. No major structural differences between the IOI and IOE fractions were observed. An alkaline polysaccharide fraction ( $>450 \mathrm{kDa}$ ) was obtained from the IOI alkali extract, and consisted mainly of $(1 \rightarrow 3)$ - and $(1 \rightarrow 6)$-linked $\beta$-Glc and $(1 \rightarrow 4)$-linked $\beta$-Xyl. Several of the fractions showed in vitro immunomodulatory effect by increasing NO production in the murine macrophage and dendritic cell lines J774.A1 and D2SC/1. Most fractions managed to increase NO production only at the highest concentration tested $(100 \mu \mathrm{g} / \mathrm{ml})$, while the neutral fraction IOE-WN activated potent NO production at $10 \mu \mathrm{g} / \mathrm{ml}$ and was considered the most promising immunomodulating fraction in this study.
\end{abstract}

\section{Introduction}

Inonotus obliquus (Fr.) Pilát, a rather unusual polypore fungus found in northern latitudes, has been used as an infusion in traditional medicine in Northern Europe and Russia since the 16th century against a wide range of illnesses such as tuberculosis, heart disease, cancer and stomach issues (Saar, 1991; Shikov et al., 2014). I. obliquus infects living, broad-leaved trees from the Betulaceae family, causing white heart rot in the tree, growing for decades while producing massive, sterile conks of sclerotia without forming fruiting bodies (Cha, Lee, Lee, \& Chun, 2011; Min-Woong et al., 2008). Scientific studies have revealed many biological properties of extracts and isolated substances from $I$. obliquus, such as anti-inflammatory, anti-tumor, antioxidative and immunomodulatory properties (Chen, Huang, Cui, \& Liu, 2015; Glamoclija et al., 2015; Hu et al., 2016; Lee, Lee, Song, Ha, \& Hong,
2014; Ma, Chen, Dong, \& Lu, 2013). Structural analysis of compounds from this fungus has revealed the presence of triterpenoids, polyphenols, melanin pigments and polysaccharides which are likely to be, at least in part, responsible for its bioactive effects (Chen et al., 2015; Hwang, Lee, \& Yun, 2016; Kim et al., 2005; Youn et al., 2009; Zhao et al., 2015; Zheng et al., 2010).

Fungal polysaccharides, for example $(1 \rightarrow 3 / 1 \rightarrow 6)$ - $\beta$-D-glucans such as lentinan from Lentinula edodes (Shiitake), have been associated with immunomodulatory and anti-tumor effects (Zhang, Cui, Cheung, \& Wang, 2007). In Japan, lentinan is used in human cancer therapy in combination with conventional treatments such as chemotherapy to improve wellbeing and treatment outcome for patients (Ina, Kataoka, \& Ando, 2013; Kapoor, 2014). Various mechanisms of action have been proposed for the anti-tumor effects of such fungal polysaccharides, with both pro- and anti-inflammatory effects being reported in the literature

\footnotetext{
* Corresponding author.

E-mail address: c.w.wold@farmasi.uio.no (C.W. Wold).
} 
(Du, Lin, Bian, \& Xu, 2015; Schwartz \& Hadar, 2014; Zhang et al., 2007). A central mechanism of action of these compounds is their interaction with pattern recognition receptors (PRRs) on innate immune cells such as macrophages and dendritic cells (Erwig \& Gow, 2016). As such, the immunological effects of the polysaccharides will depend greatly on the specific receptors involved, since different PRRs could induce different downstream effects in the cell when activated (Fraser, Stuart, \& Ezekowitz, 2004). In this regard the structure and shape of the polysaccharide, including monosaccharide composition, molecular weight $\left(\mathrm{M}_{\mathrm{w}}\right)$, branching and anomeric configuration, are important for its activity and could explain some of the conflicting reports in the scientific literature. Further, many alleged polysaccharide extracts from medicinal fungi are shown to contain high levels of polyphenols and proteins (Wei \& Van Griensven, 2008). Since some fungal compounds can activate immune cells while others can suppress the same cells a proper purification of the extracts and isolation of pure compounds are highly important to avoid additional scientific confusion (Lu et al., 2016; Wei \& Van Griensven, 2008).

Although there are several reports of polysaccharides from $I . o b$ liquus with immunomodulatory and anti-tumor activity (Chen et al., 2015; Fan, Ding, Ai, \& Deng, 2012; Kim et al., 2006; Lee et al., 2014; Rhee, Cho, Kim, Cha, \& Park, 2008), satisfactory characterization of the polysaccharides including anomeric configuration and linkage analysis is lacking, and the purification methods used are often questionable. To our knowledge, the present study is the first extensive characterization of $I$. obliquus immunomodulating polysaccharides.

\section{Methods}

\subsection{Preparation of fungal material}

A fresh Inonotus obliquus (family: Hymenochaetaceae) specimen was harvested from a birch tree in Oslo, Norway and verified by Prof. Klaus Høiland (Dept. of Biosciences, UiO, Norway). A voucher specimen was deposited in the Pharmacognosy section, School of Pharmacy, University of Oslo, Norway. The fungal material was separated into two parts distinguished by the difference in texture and color - a brown interior part and a black exterior part - before cut into small pieces and lyophilized. The separate parts were then ground to a finely dispersed powder using an industrial blender (RAW $\left.{ }^{\circledR} \mathrm{X} 1500\right)$.

\subsection{Extraction of polysaccharides}

In order to remove low molecular weight compounds, the fungal material (weight: $227 \mathrm{~g} / 131 \mathrm{~g}$ interior/exterior) was pre-extracted in a soxhlet extractor, first with dichloromethane (DCM) for $48 \mathrm{~h}$ followed by methanol $(\mathrm{MeOH})$ for $48 \mathrm{~h}$. The remaining dried residue was then extracted first with distilled water $\left(\mathrm{dH}_{2} \mathrm{O}, 100{ }^{\circ} \mathrm{C}, 3 \mathrm{x}, 2 \mathrm{~h}\right)$ and subsequently with boiling sodium hydroxide plus sodium borodeuteride under reflux ( $1 \mathrm{M} \mathrm{NaOH}, 0.135 \mathrm{M} \mathrm{NaBD}_{4}, 2 \mathrm{x}, 4 \mathrm{~h}$ ). After each extraction, the supernatant was collected after centrifugation $(4000 \mathrm{rpm}$, $20 \mathrm{~min}$ ) and concentrated under reduced pressure on a rotavapor. The extracts were then treated with pancreatin $\left(3 \mathrm{~h}, 37^{\circ} \mathrm{C}\right.$, Sigma-Aldrich $\left.{ }^{\circledR}\right)$ to degrade proteins, before ethanol precipitation $\left(70 \%, 48 \mathrm{~h}, 4^{\circ} \mathrm{C}\right)$ and centrifugation. The precipitates were finally dissolved in $\mathrm{dH}_{2} \mathrm{O}$, dialyzed (cut-off $3500 \mathrm{Da}, 72 \mathrm{~h}, 4^{\circ} \mathrm{C}$ ) and lyophilized to obtain four crude polysaccharide extracts - two water-soluble extracts termed IOI-W and IOE-W (Inonotus obliquus Interior/Exterior Water-extracted) and two alkali-soluble extracts termed IOI-A and IOE-A (Inonotus obliquus Interior/Exterior Alkali-extracted).

\subsection{Fractionation of polysaccharides}

The extraction and fractionation scheme used to obtain the purified polysaccharide fractions is presented in Fig. 1. All column chromatography experiments were carried out using an Äkta FPLC system
(Pharmacia Äkta, Amersham Pharmacia Biotech, Uppsala, Sweden) with a fraction collector. All extracts and fractions were filtered through $0.45 \mu \mathrm{m}$ Millipore filters before loaded onto the columns. The Unicorn 4.0 software (GE Healthcare, Uppsala, Sweden) was used to set-up and monitor the experiments.

\subsubsection{Anion-exchange chromatography}

The water-soluble crude extracts IOI-W and IOE-W $(10 \mathrm{mg} / \mathrm{ml})$ were applied to a column packed with ANX Sepharose ${ }^{\mathrm{TM}} 4$ Fast Flow (high sub) (GE Healthcare). Neutral fractions were obtained by eluting with $\mathrm{dH}_{2} \mathrm{O}(2 \mathrm{ml} / \mathrm{min})$, while acidic fractions were obtained using a linear $\mathrm{NaCl}$ gradient $(0-1.5 \mathrm{M}, 2 \mathrm{ml} / \mathrm{min})$. Fractions were collected $(15 \mathrm{ml} /$ tube) and carbohydrate elution profiles were monitored using the phenol-sulfuric acid method (Dubois, Gilles, Hamilton, Rebers, \& Smith, 1956). The related fractions were pooled, dialyzed against $\mathrm{dH}_{2} \mathrm{O}$ (cutoff $3500 \mathrm{Da}$ ) and lyophilized.

\subsubsection{Size exclusion chromatography}

Several fractions were subjected to gel filtration/size exclusion column chromatography (SEC) using different SEC columns. The acidic fraction from IOI-W, termed IOI-WAc $(20 \mathrm{mg} / \mathrm{ml})$, was fractionated by Hiload $^{\mathrm{TM}}$ 16/60 Superdex ${ }^{\mathrm{TM}} 200$ prep grade column (GE Healthcare), eluting with $10 \mathrm{mM} \mathrm{NaCl}$ at $0.5 \mathrm{ml} / \mathrm{min}$ and collected ( $2 \mathrm{ml} /$ tube). The neutral fractions IOI-WN and IOE-WN $(2 \mathrm{mg} / \mathrm{ml})$ were subjected to SEC using a Superose ${ }^{\circledast} 6$ prep grade column (GE Healthcare), eluting with $10 \mathrm{mM} \mathrm{NaCl}$ at $0.3 \mathrm{ml} / \mathrm{min}$ and collected ( $0.5 \mathrm{ml} /$ tube).

The alkali-soluble crude extracts IOI-A and IOE-A $(20 \mathrm{mg} / \mathrm{ml})$ were subjected to SEC using a Sephacryl S-500 h column (GE Healthcare), eluting with $0.2 \mathrm{M} \mathrm{NaOH}$ at $2 \mathrm{ml} / \mathrm{min}$ and collected $(15 \mathrm{ml} / \mathrm{tube}$ ). Ultimately, carbohydrate elution profiles were monitored after each experiment using the phenol-sulfuric acid method and related fractions were pooled, dialyzed and lyophilized.

\subsection{Molecular weight determination by SEC-MALLS}

Molecular weight distributions and averages, notably the average molecular weights $\left(\mathrm{M}_{\mathrm{w}}\right)$ of the polysaccharides, were determined as previously described (Austarheim et al., 2012). Briefly, two SEC columns were coupled in series, coupled to a SIL-10AF auto sampler, a LC10ADvp pump and a SCL-10Avp system controller (Shimadzu, Japan). Three online detectors were used, a multi-angle laser light scattering (MALLS) Dawin Heleos-II (Wyatt, USA), a refractive index detector (Optilab DSP, Wyatt) and a viscometer (ViscoStar, Wyatt). The column was eluted with aqueous $0.05 \mathrm{M} \mathrm{Na}_{2} \mathrm{SO}_{4} / 0.01 \mathrm{M}$ EDTA, pH 6 at a flow rate of $0.5 \mathrm{~mL} / \mathrm{min}$. Pullulan $\mathrm{P} 8(8 \mathrm{kDa})$ was used for normalization of all the MALLS detectors. $100-150 \mu \mathrm{l}$ sample solution $(0.4-1.5 \mathrm{mg} / \mathrm{mL})$ was injected. All samples were dissolved in distilled water $24 \mathrm{~h}$ prior to analysis, and diluted with concentrated eluent to reach eluent concentration before injection. The samples were run in duplicates, and $M_{w}$ for each sample was calculated using the ASTRA V software (Wyatt). A refractive index increment $(\mathrm{dn} / \mathrm{dc})_{\mu}$ of $0.15 \mathrm{ml} / \mathrm{g}$ was used in the calculations.

\subsection{Determination of proteins, phenolics and lipopolysaccharide in the polysaccharide fractions}

The protein content of the purified polysaccharide fractions ( $1 \mathrm{mg}$ ) was determined by the Bio-Rad protein assay (Bio-Rad), based on the method of Bradford (Bradford, 1976). The standard procedure for microtiter plates was used with bovine serum albumin (BSA, Sigma) as a protein standard $(30-500 \mu \mathrm{g} / \mathrm{ml})$. The experiment was carried out in triplicates.

The amount of phenolic compounds in the purified polysaccharide fractions was determined using the Folin-Ciocalteu assay (Swain \& Hillis, 1959). Briefly, the samples were dissolved in $\mathrm{dH}_{2} \mathrm{O}(1 \mathrm{mg} / \mathrm{ml})$ before adding the same amount of Folin-Ciocalteu's phenol reagent (1:2 




Fig. 1. Flowchart for extraction and fractionation of polysaccharides from the interior part of Inonotus obliquus.

in $\mathrm{dH}_{2} \mathrm{O}$, Merck/Kebo), mixed and left for $3 \mathrm{~min}$ at room temperature. $1 \mathrm{M} \mathrm{Na} 2 \mathrm{CO} 3$ was added, before the tubes were mixed and allowed to stand for $1 \mathrm{~h}$. The absorbance was measured at $750 \mathrm{~nm}$. A standard curve was plotted using ferulic acid $(0-50 \mu \mathrm{g} / \mathrm{ml})$. The total phenolic content was determined as ferulic acid equivalents. The experiment was carried out in triplicates.

The amount of lipopolysaccharide (LPS) contamination in the purified fractions was determined by a previously described method (de Santana-Filho et al., 2012), with some modifications. Briefly, the lyophilized polysaccharide samples $(5 \mathrm{mg})$ were dissolved in $3 \mathrm{M} \mathrm{HCl}$ in $\mathrm{MeOH}$ and incubated for $20 \mathrm{~h}$ at $80^{\circ} \mathrm{C}$. The solution was then partitioned between hexane $(1 \mathrm{ml})$ and $\mathrm{dH}_{2} \mathrm{O}(0.5 \mathrm{ml})$ three times. The hexane phase was collected, pooled and dried under a gentle $\mathrm{N}_{2}$ stream. The samples were then acetylated at $100{ }^{\circ} \mathrm{C}$ for $1 \mathrm{~h}$ using a $1: 1$ mixture of pyridine and acetic anhydride, and thoroughly dried. Finally, acetone $(70 \mu \mathrm{l})$ was added and the samples were analyzed by GC-MS, using the GC-MS solution software, Version 2.10 (Shimadzu Corporation). The detection limit of this assay was $\sim 2 \mathrm{ng} / \mu \mathrm{l}$.

\subsection{Monosaccharide composition}

The monosaccharide composition of the crude extracts and purified fractions was determined using a method originally described by Chambers and Clamp with some modifications (Chambers \& Clamp, 1971; Nyman, Aachmann, Rise, Ballance, \& Samuelsen, 2016). In short, the samples were subjected to methanolysis with $3 \mathrm{M}$ hydrochloric acid $(\mathrm{HCl})$ in anhydrous methanol $(\mathrm{MeOH})$ for $24 \mathrm{~h}$ at $80^{\circ} \mathrm{C}$. To analyze for the presence of amino sugars, the samples were then dried using nitrogen before pyridine and acetic acid anhydride were added for $3 \mathrm{~min}$. After drying, $0.5 \mathrm{M} \mathrm{HCl}$ in $\mathrm{MeOH}$ was added and the samples were incubated for $1 \mathrm{~h}$ at $65^{\circ} \mathrm{C}$. Trimethylsilylated (TMS) derivatives of the methyl glycosides obtained after methanolysis were analyzed by capillary gas chromatography on a Trace ${ }^{\mathrm{TM}} 1300$ GC (Thermo Scientific ${ }^{\mathrm{TM}}$ ). Mannitol was used as an internal standard.

\subsection{Linkage analysis by methylation/GC-MS}

Glycosidic linkage analysis was performed by methylation and subsequent gas chromatography-Mass spectrometry (GC-MS) based on previously described methods (Ciucanu \& Kerek, 1984; Pettolino, Walsh, Fincher, \& Bacic, 2012). Briefly, polysaccharide samples $\left(\sim 1 \mathrm{mg}\right.$ ) were first reduced using $\mathrm{NaBD}_{4}$ to convert uronic acids to their corresponding neutral sugars. The polymers were then methylated with methyl iodide after dissolving in dimethylsulfoxide (DMSO) and $\mathrm{NaOH}$. The per-O-methylated polysaccharides were hydrolyzed with trifluoroacetic acid (TFA, $2.5 \mathrm{M}, 4 \mathrm{~h}$ ) and reduced with $\mathrm{NaBD}_{4}$. The $O$ methylated alditols were acetylated by adding 1-methylimidazole and acetic anhydride and partially $\mathrm{O}$-methylated alditol acetates were finally extracted with DCM and analyzed on a GCMS-QP2010 (Shimadzu Corporation) with a Restek Rxi-5MS silica column $(30 \mathrm{~m}$, i.d. $0.25 \mathrm{~mm}$, $0.25 \mu \mathrm{m}$ film thickness), split injection and set at a constant pressure mode. Initial flow was $1 \mathrm{ml} / \mathrm{min}$. The injector and interface temperatures were $280{ }^{\circ} \mathrm{C}$. At the time of injection, the column temperature was $80{ }^{\circ} \mathrm{C}$, then, after $5 \mathrm{~min}$, the temperature was increased with $10^{\circ} \mathrm{C} / \mathrm{min}$ up to $140{ }^{\circ} \mathrm{C}$ followed by $4{ }^{\circ} \mathrm{C} / \mathrm{min}$ to $210^{\circ} \mathrm{C}$ and then $20^{\circ} \mathrm{C} / \mathrm{min}$ to $310^{\circ} \mathrm{C}$, at which it was kept for $4 \mathrm{~min}$. Ion source temperature was $200^{\circ} \mathrm{C}$. Helium was used as the carrier gas. Spectra were analyzed using GC-MS solution software, Version 2.10 (Shimadzu Corporation).

\subsection{Characterization of a $(1 \rightarrow 6)-\alpha-3-O$-methyl-galactose}

\subsubsection{Demethylation of monosaccharides using boron tribromide}

The GC chromatograms from the monosaccharide composition analysis revealed three large peaks with unknown identity in some of the samples, which were speculated to come from a methylated hexose. To characterize this unknown compound, a method described in Methods in Carbohydrate Chemistry, Vol II (Whistler \& Wolfrom, 1963) was used. Briefly, IOE-WN was methanolyzed as described in Section 2.6, before the residue was dissolved in DCM and cooled to $-80^{\circ} \mathrm{C}$ using a mixture of acetone and dry ice. Boron tribromide $\left(\mathrm{BBr}_{3}\right.$, $-80^{\circ} \mathrm{C}$ ) was then carefully added to the sample, and left at this temperature for $30 \mathrm{~min}$ to induce demethylation. The samples were then left at room temperature overnight with a drying tube with $\mathrm{CaCl}_{2}$, before washed twice with $\mathrm{MeOH}$, dried and analyzed by GC as described in Section 2.6. Finally, the chromatogram from the demethylated sample was compared with the untreated counterpart. The standard 
curve from galactose was used for quantification.

\subsubsection{Linkage analysis by ethylation}

To establish the type of linkages and position of the methyl group, IOE-WN was subjected to ethylation and GC-MS analysis, as described in Section 2.7 with one modification; ethyl iodide was used instead of methyl iodide, which would produce unique fragmentation patterns in the GC-MS spectra.

\subsection{IR spectroscopy}

Infrared (IR) spectra of the purified polysaccharide fractions were obtained on a Nicolet $^{\mathrm{TM}}$ FT-IR Spectrometer (Thermo Fischer Scientific). In short, dried samples $\left(\sim 2 \mathrm{~mm}^{3}\right)$ were pressed onto an attenuated total reflectance (ATR) crystal using a high pressure tower to provide consistent results. The results were analyzed using the OMNIC8 software.

\subsection{Nuclear magnetic resonance (NMR) spectroscopy}

The water-extracted polysaccharide fractions $(5-10 \mathrm{mg})$ were dissolved in $500 \mu \mathrm{D}_{2} \mathrm{O}$ (99.9\%, Sigma). All experiments were carried out on a Bruker Avance III (799.90 MHz for $1 \mathrm{H}$ and $201.14 \mathrm{MHz}$ for ${ }^{13} \mathrm{C}$ ) equipped with a $5 \mathrm{~mm}$ TCI ${ }^{1} \mathrm{H} /\left({ }^{13} \mathrm{C},{ }^{15} \mathrm{~N}\right)$ cryoprobe using acetone as reference (2.22 ppm and $30.89 \mathrm{ppm}$ for ${ }^{1} \mathrm{H}$ and ${ }^{13} \mathrm{C}$, respectively). The chemical shifts were assigned using the following spectra: $1 \mathrm{D}{ }^{1} \mathrm{H}$ with presaturation, $1 \mathrm{D}{ }^{13} \mathrm{C}, 2 \mathrm{D}$ double quantum filter correlated spectroscopy (DQF-COSY), 2D total correlation spectroscopy (TOCSY) with $60 \mathrm{~ms}$ mixing time, 2D nuclear Overhauser effect spectroscopy (NOESY) with $200 \mathrm{~ms}$ mixing time, $2 \mathrm{D}{ }^{13} \mathrm{C}$ heteronuclear single quantum coherence (HSQC) with multiplicity editing, 2D ${ }^{13} \mathrm{C}$ HSQC- $\left[{ }^{1} \mathrm{H},{ }^{1} \mathrm{H}\right]$ TOCSY (HSQC-TOSCY) with $60 \mathrm{~ms}$ mixing time, and $2 \mathrm{D}{ }^{13} \mathrm{C}$ heteronuclear multi-bond correlation (HMBC) optimized for $10 \mathrm{~Hz}$ long range coupling constants. All spectra were recorded at $313 \mathrm{~K}$. Spectra were recorded using TopSpin 3.5 and processed and analyzed using the TopSpin 3.5 software (Bruker).

The alkali-extracted fraction IOI-A1 $(10 \mathrm{mg})$ was dissolved in $0.1 \mathrm{M}$ $\mathrm{NaOH}$ with $\mathrm{D}_{2} \mathrm{O}$ (99.9\%, Sigma). Experiments were carried out on a Bruker Avance III HD Ascend (800.03 MHz for ${ }^{1} \mathrm{H}$ and $201.17 \mathrm{MHz}$ for ${ }^{13} \mathrm{C}$ ) equipped with a $5 \mathrm{~mm}$ TCI ${ }^{1} \mathrm{H} /\left({ }^{13} \mathrm{C},{ }^{15} \mathrm{~N}\right)$ cryoprobe using the internal standard TMSP-d4 3-(trimethylsilyl)-2,2,3,3-tetradeuteropropionic acid sodium salt as reference. The chemical shifts were assigned using the following spectra: $1 \mathrm{D}{ }^{1} \mathrm{H}$ with presaturation and excitation sculpting solvent suppression, $1 \mathrm{D}^{13} \mathrm{C}, 2 \mathrm{D}$ DQF-COSY, 2D TOCSY with $80 \mathrm{~ms}$ mixing time, 2D NOESY with $300 \mathrm{~ms}$ mixing time and $2 \mathrm{D}{ }^{13} \mathrm{C}$ HSQC with multiplicity editing. Spectra were recorded at $313 \mathrm{~K}$ and acquired, processed and analyzed using TopSpin 3.5.

\subsection{Periodate oxidation and Smith degradation}

Periodate oxidation and Smith degradation were carried out as previously described (Kamerling \& Gerwig, 2007). In short, IOE-WN and IOI-WAc $(15 \mathrm{mg})$ were oxidized with $0.05 \mathrm{M}$ sodium periodate $(3 \mathrm{~mL})$ and $0.2 \mathrm{M}$ acetate buffer $\mathrm{pH} 4.0(3 \mathrm{~mL})$ at $4{ }^{\circ} \mathrm{C}$ in the dark with stirring for $48 \mathrm{~h}$. The oxidation was terminated by addition of $1 \%$ ethylene glycol and kept for $1 \mathrm{~h}$ before desalting using a PD-10 column (GE Healthcare). The samples were reduced with $\mathrm{NaBH}_{4}(60 \mathrm{mg})$ and left at room temperature overnight. The reaction mixture was neutralized to $\mathrm{pH} 7.0$ with $1 \mathrm{M}$ acetic acid on ice and desalted again before hydrolysis with $0.05 \mathrm{M}$ TFA for $30 \mathrm{~min}$ at $100{ }^{\circ} \mathrm{C}$. The samples were neutralized using $1 \mathrm{M} \mathrm{NaOH}$ and analyzed and fractionated using a CarboPac $^{\mathrm{TM}}$ PA100 ZOU column (Dionex, ThermoFisher) with galactose, glucose, maltotetraose and maltohexaose as standards (Sigma). Finally the fractions were collected and subjected to methanolysis and GC analysis as described in Section 2.6.

\subsection{Cell lines}

The murine macrophage cell line J774.A1 (a gift from Anders Gammelsrud, Norwegian Veterinary Institute) and the murine dendritic cell line D2SC/1 (a gift from Francesca Granucci, University of Milan) were grown in RPMI medium with 10\% FBS, 5\% streptomycin/penicillin, $1 \%$ NEAA and $1 \%$ mercaptoethanol, and incubated at $37^{\circ} \mathrm{C}, 5 \%$ $\mathrm{CO}_{2}$. Prior to the experiments both cell lines were tested and found to be negative for Mycoplasma infection.

\subsection{Nitric oxide assay}

Nitric oxide (NO) production by activated immune cells was measured using the Griess reagent system (Promega). Briefly, J774.A1 and D2SC/ 1 cells were seeded at a density of $5 \times 10^{5}$ cells $/ \mathrm{ml}$ in flat-bottomed 96-well plates to a total volume of $100 \mu \mathrm{l}$, and stimulated for $24 \mathrm{~h}$ with increasing concentrations of polysaccharide samples together with interferon-gamma $(20 \mathrm{ng} / \mathrm{ml}$, recombinant murine IFN- $\gamma$, Peprotech). After $24 \mathrm{~h}$, the supernatant was collected and Griess reagents $\mathrm{A}$ and $\mathrm{B}$ were added to convert $\mathrm{NO}$ into nitrite $\left(\mathrm{NO}_{2}{ }^{-}\right)$, which could be quantified colorimetrically using a dilution series of $\mathrm{NaNO}_{2}$ as a standard curve. The absorbance was measured at $540 \mathrm{~nm}$. LPS $(1 \mu \mathrm{g} /$ ml, from E.coli 055:B5, Sigma) and Pam3CSK4 (2 $\mu \mathrm{g} / \mathrm{ml}$, InvivoGen) with and without IFN- $\gamma(20 \mathrm{ng} / \mathrm{ml})$, IFN- $\gamma$ alone and untreated cells were used as controls. The nitrite value from the untreated negative control was set to zero and the value was subtracted from the other samples. The experiments were carried out in duplicates, and repeated two (D2SC/1) or three (J774.A1) times.

\section{Results and discussion}

\subsection{Extraction and fractionation of polysaccharides}

I. obliquus was separated into an interior and exterior part and extracted using a stepwise procedure with solvents of increasing polarity (Fig. 1). The extracts obtained by DCM and $\mathrm{MeOH}$ extraction were saved for later studies. Water- and alkali extraction, protease treatment and ethanol precipitation of the remaining fungal mass gave the four crude polysaccharide extracts, IOI-W, IOE-W, IOI-A and IOE-A. Since this procedure is a widely accepted method for isolation of crude polysaccharides from plants and fungi, it was expected that the main component in these extracts was polysaccharides (Bouchard, Hofland, \& Witkamp, 2007; Zhang et al., 2007). However, all four crude extracts appeared dark brown in color, indicating presence of substances other than polysaccharides. I. obliquus is known to contain massive amounts of melanin pigments, reported to be the main content in I. obliquus water extracts (Shashkina, Shashkin, \& Sergeev, 2006). One study reported a $57 \mathrm{kDa}$ allomelanin pigment isolated from $I$. obliquus, which could explain why the color remained in the extracts even after dialysis and ethanol precipitation (Kukulyanskaya, Kurchenko, Kurchenko, \& Babitskaya, 2002). Indeed, the fractions obtained after anion-exchange chromatography of the crude water-extracts revealed that only a minor part of the extracts were polysaccharides. The crude extract IOI-W ( $3.2 \mathrm{~g})$ gave two polysaccharide fractions - IOI-WN $(80 \mathrm{mg})$ and IOIWAc $(75 \mathrm{mg})$ - which combined were responsible for $4.8 \%$ of the total extract weight, or $0.45 \%$ of total interior I. obliquus dry weight. Although most of the pigment was trapped in the ANX sepharose resin when eluting with water, some of the color came out with the acidic fractions IOI-WAc and IOE-WAc. The presence of phenolics was later detected in the acidic fractions (Table 1). Previous studies have reported fungal melanin to have a polyphenolic structure (Eisenman \& Casadevall, 2012; Prados-Rosales et al., 2015), and in agreement with this phenolics were not detected in the fractions where the color was absent, such as IOI-WN. After anion-exchange chromatography, IOIWN, IOE-WN and IOI-WAc were subjected to SEC. IOI-WAc was fractionated using Superdex 200, giving the fractions IOI-WAcF1, IOI- 
Table 1

$\mathrm{M}_{\mathrm{w}}, \mathrm{R}_{\mathrm{G}}$, carbohydrate content, protein content (expressed as albumin), total phenolic content (expressed as ferulic acid) and LPS content in the isolated polysaccharide fractions.

\begin{tabular}{|c|c|c|c|c|c|c|}
\hline Fraction & $\mathrm{M}_{\mathrm{w}}(\mathrm{kDa})$ & $\mathbf{R}_{\mathrm{G}}(\mathrm{nm})$ & Total carbohydrate (\%) & Total protein (\%) & Total phenolics (\%) & LPS contamination \\
\hline IOI-WN & 60 & $57^{\mathrm{a}}$ & 89 & $<0.1$ & $<0.1$ & n.d. \\
\hline IOI-WAc & 15 & $<10-15^{\mathrm{b}}$ & 69.1 & $<0.1$ & 6.7 & n.d. \\
\hline IOI-WAcF1 & 28 & 17 & 81.9 & $<0.1$ & 4.2 & n.d. \\
\hline IOI-WAcF2 & 14 & $<10-15^{\mathrm{b}}$ & 61.2 & $<0.1$ & 5.8 & n.d. \\
\hline IOI-WAcF3 & 10 & $<10-15^{\mathrm{b}}$ & 58.3 & $<0.1$ & 9.7 & n.d. \\
\hline IOE-WN & 73 & 36 & 54.7 & $<0.1$ & $<0.1$ & n.d. \\
\hline IOE-WAc & 31 & $29^{\mathrm{a}}$ & 47.3 & $<0.1$ & 2.0 & $0.16 \%$ \\
\hline IOI-A1 & $>450$ & n.e. & 41.9 & $<0.1$ & 0.4 & n.d. \\
\hline
\end{tabular}

n.e. = not established; n.d. = not detected; Limit of detection for LPS: $2 \mathrm{ng} / \mu \mathrm{l}$ (de Santana-Filho et al., 2012).

${ }^{a}$ May be grossly overestimated due to irregular light scattering data.

b below detection limit.

WAcF2 and IOI-WAcF3 in that order (Fig. S30). The other samples could not be fractionated further (Figs. S27-S29).

Alkaline extraction of fungal polysaccharides has been reported to be a promising method that could increase polysaccharide yields (Huang et al., 2010). We have previously optimized a method in our lab for this extraction method by adding sodium borodeutride which could protect the reducing ends and thus prevent the polymers from degrading (see Section 2.2 for details). However, the downside to alkaline extraction is that it could also cause degradation of labile side-groups in the polymer such as methyl- and acetyl groups (unpublished results). The alkali-extracted interior part yielded a relatively pure polysaccharide fraction after SEC - IOI-A1 - accounting for $12.5 \%$ of the alkaline crude extract ( $\sim 1 \%$ of total dry interior weight). In contrast, the extract from the exterior part yielded only heavily pigmented fractions after SEC with low amounts of polysaccharide (IOE-A1 and IOE-A2) and these were not analyzed further in this study. The presence of melanin in the polysaccharide extracts highlights the importance of proper fractionation when isolating polysaccharides from I. obliquus, especially if biological assays will be conducted.

\subsection{Molecular weight determination by SEC-MALLS}

The weight-average molecular weights $\left(\mathrm{M}_{\mathrm{w}}\right)$ of the polysaccharide fractions and weight average radius of gyration $\left(R_{G}\right)$ values were determined by SEC-MALLS analysis (Table 1, Figs. S19-S26). The neutral polysaccharides IOI/IOE-WN had higher $\mathrm{M}_{\mathrm{w}}$ than the acidic ones (60/ $73 \mathrm{kDa}$ vs $10-31 \mathrm{kDa}$ ), while the alkaline-extracted IOI-A1 gave the highest $\mathrm{M}_{\mathrm{w}}$ of $\sim 2000 \mathrm{kDa}$. However, the $\mathrm{M}_{\mathrm{w}}$ of IOI-A1 must be interpreted with caution, since the SEC-MALLS analysis was conducted at $\mathrm{pH}$ 6 while IOI-A1 is poorly soluble at $\mathrm{pH}<8$. An HPLC analysis of this sample was therefore carried out in addition to SEC-MALLS, dissolving IOI-A1 in $10 \mathrm{mM} \mathrm{NaOH}$. The HPLC experiment indicated a polymer size larger than the biggest dextran standard utilized, which was $450 \mathrm{kDa}$ (data not shown). IOI-WN and IOE-WAc were suggested to contain more than one type of polymer, but when using prep grade SEC it was not possible to fractionate these any further. IOI-WAc was also suggested to contain more than one polymer. In the SEC-MALLS chromatogram of IOI-WAc (Fig. S21), only one main peak can be observed. However, the peak was quite broad, indicating that it could contain polymers of more than one size. This seemed to be the case, as IOI-WAc was fractionated into the three sub-fractions IOI-WAcF1-F3 by gel filtration (Fig. 1). In general, the SEC chromatograms, notably the light scattering profiles, were too irregular for conventional data analysis, making it difficult to estimate the radius of gyration $\left(R_{G}\right)$ values, as well as the number-average molecular weights $\left(M_{n}\right)$. Nevertheless, the $R_{G}$ (weight average) ranges observed across the peaks indicated that the polymers were in some cases rather compact structures, possibly aggregates, which could explain why we could not manage to further fractionate IOI-WN and IOE-WAc by SEC. There are a few reports in the literature on molecular weight of polysaccharides from I. obliquus. One group found a $48.8 \mathrm{kDa}$ water-soluble polysaccharide from wild $I$. $o b$ liquus, in reasonable agreement with our own data (Chen et al., 2015). Another study reported water-soluble $I$. obliquus polysaccharides with a wide range of sizes, from < $10 \mathrm{kDa}$ up to $1.100 \mathrm{kDa}$ (Kim et al., 2005), but the use of cultivated $I$. obliquus mycelium instead of wild fungus makes it difficult to compare these results directly with our own.

\subsection{Purity of the polysaccharide fractions and LPS contamination}

Table 1 gives an overview of the purity of the polysaccharide fractions, showing carbohydrate content, protein content, phenolic content and LPS contamination in the fractions. The fractions had a carbohydrate content ranging from 41.9 to $89 \%$. The percentages are based on the GC analysis, using mannitol as internal standard. It should be noted that there is some uncertainty to the calculations of these percentages. The peak coming from glucose in the GC chromatogram was too big for accurate quantification, possibly leading to an underestimation of actual content of this monosaccharide, but when diluting the samples the peaks from several of the other monosaccharides became too small. In addition, the calculation for $3-\mathrm{O}-\mathrm{Me}-\mathrm{Gal}$ was based on the galactose standard curve, meaning it could have been inaccurately quantified. All fractions were essentially free from proteins, as would be expected since the extracts were treated with a protease prior to fractionation. The phenolic content was likely related to the presence of melanin pigments as discussed in Section 3.1. The acidic fractions had a higher content of phenolics than the neutral fractions. For example, IOI-WN did not contain any phenolics, while IOI-WAc had a phenolic content of $6.7 \%$. This suggests that the pigments are not covalently bound to the polysaccharides, although a possible explanation could be that the galacturonic acid (which is present in large amounts in the acidic fractions) interacts with the pigments in some way. When IOI-WAc was further fractionated with SEC, the three sub fractions IOI-WAcF1- F3 contained 4,2\%, 5.8\% and $9.7 \%$ phenolics, respectively, suggesting that the melanin pigments range in size from at least $10-31 \mathrm{kDa}$. The alkali extracted IOI-A1 also contained some phenolic traces, accounting for $0.4 \%$ of total sample weight. The samples were analyzed for the presence of LPS, a constituent of the outer cell membrane in gram-negative bacteria and a common contaminant in natural products research (Lieder, Petersen, \& Sigurjonsson, 2013). Only the acidic fraction IOEWAc from the exterior part was found to contain LPS, accounting for $0.16 \%$ of the total sample weight which is equivalent to $1.6 \mu \mathrm{g} / \mathrm{ml} \mathrm{ng} /$ $\mathrm{ml}$ LPS in a $1 \mathrm{mg} / \mathrm{ml}$ sample. Biological assays are often highly sensitive to LPS, and it has been reported that LPS at less than $1 \mathrm{ng} / \mathrm{ml}$ can be recognized by human immune cells (Lieder et al., 2013). This underlines the need for caution when interpreting results when using natural products in such assays, and the analysis for the presence/absence of LPS should always be carried out prior to the experiments. 
Table 2

Monosaccharide composition (mol \%) after methanolysis and GC of the polysaccharide fractions isolated from I. obliquus.

\begin{tabular}{|c|c|c|c|c|c|c|c|c|}
\hline Monosaccharide & IOI-WN & IOE-WN & IOI-WAc & IOI-WAcF1 & IOI-WAcF2 & IOI-WAcF3 & IOE-WAc & IOI-A1 \\
\hline Arabinose & 1.4 & 2.0 & 2.1 & 2.3 & 2.3 & 2.4 & 0.9 & n.d. \\
\hline Rhamnose & 1.3 & 1.3 & 5.1 & 4.8 & 5.4 & 7.7 & 3.4 & n.d. \\
\hline Fucose & 1.7 & 2.5 & 1.3 & 1.5 & 1.0 & 0.7 & 0.5 & 2.8 \\
\hline Xylose & 6.9 & 8.9 & 8.8 & 4.7 & 5.2 & 19.0 & 6.0 & 12.2 \\
\hline Mannose & 6.8 & 7.2 & 8.2 & 10.8 & 13.4 & 4.8 & 8.4 & 4.0 \\
\hline Galactose & 17.5 & 17.2 & 11.2 & 13.5 & 9.6 & 8.1 & 15.6 & n.d. \\
\hline Glucose & 53.1 & 49.3 & 37.9 & 34.3 & 43.1 & 39.2 & 31.6 & 78.2 \\
\hline Glucuronic acid & 1.0 & 2.1 & 5.1 & 4.4 & 4.0 & 4.4 & 3.1 & n.d. \\
\hline Galacturonic acid & 1.8 & 1.2 & 15.7 & 17.7 & 13.1 & 12.9 & 24.3 & n.d. \\
\hline 3-O-methyl galactose & 8.7 & 8.3 & 4.6 & 6.0 & 2.9 & 1.1 & 6.2 & n.d. \\
\hline $\mathrm{N}$-acetylglucosamine & n.d. & n.d. & n.d. & n.d. & n.d. & n.d. & n.d. & 2.8 \\
\hline Sum \% & 100 & 100 & 100 & 100 & 100 & 100 & 100 & 100 \\
\hline
\end{tabular}

(n.d. = not detected)

\subsection{Monosaccharide composition}

The monosaccharide composition of the polysaccharide fractions is presented in Table 2. The most common monosaccharide in all watersoluble fractions was glucose, with galactose, mannose and xylose present in substantial amounts. The acidic fractions had high levels of galacturonic acid and increased amounts of rhamnose compared to the neutral fractions, while arabinose, fucose and glucuronic acid were present in minor amounts in all fractions. 3-O-methyl-galactose (3-OMe-Gal) was found in varying amounts in all fractions, and the amount decreased with decreasing size of the polymer, as seen by comparing the acidic fractions IOI-WAcF1, F2 and F3 (Tables 1 and 2). The alkaliextracted IOI-A1 contained mainly glucose (79.2\%) and xylose (12.1\%), with traces of mannose, fucose and $\mathrm{N}$-acetylglucosamine (GlcNAc). There are a few publications on isolated and purified I. obliquus polysaccharides. Chen et al. isolated a polysaccharide consisting of rhamnose, arabinose, glucose and galactose in a molar ratio of 2.5:4.6:1.0:2.6 with approx. 30\% uronic acid (Chen et al., 2015), while Fan et al. isolated a polysaccharide consisting of rhamnose, mannose and glucose in ratio 1.0:2.3:1.7 with 7.5\% uronic acid (Fan et al. 2012). The high amounts of arabinose and rhamnose in these studies are in contrast to our own data. There are several possible explanations for the differences, such as isolation methods, harvest location and age of the fungus, and which part of the fungus was used. Since arabinose and rhamnose are typical plant monosaccharides (Liu, Willför, \& Xu, 2015), it could also be possible that the I. obliquus samples were "contaminated" with birch material after harvesting, which is quite common when collecting wild-grown I. obliquus sclerotia. Apart from these papers, most of the studies on I. obliquus polysaccharides have been carried out using cultured mycelium rather than wild-grown fungi, and the results are therefore not directly comparable (Kim et al., 2006; Xu, Quan, \& Shen, 2015).

\subsection{Chemical determination of $(1 \rightarrow 6)-\alpha-3-O-M e-G a l$}

The results from the monosaccharide composition analysis by GC revealed three unknown peaks in the chromatogram, which based on previous discoveries in our lab was suggested to come from a methylated hexose (Fig. 2). To identify the unknown compound, IOE-WN was demethylated using $\mathrm{BBr}_{3}$ and analyzed by GC after methanolysis before compared with the original GC data. The three peaks in question disappeared after demethylation, while the ratio between mannose, galactose and glucose had changed from $\sim 1: 2: 7.5$ to $\sim 1: 3: 6$, suggesting that the methylated species was a galactose. The IOE-WN fraction was ethylated and analyzed by GC-MS to find the position of the methyl group. From the linkage analysis data (Table 3 ) it was seen that most of the galactose was $(1 \rightarrow 6)$-linked, and therefore we searched for fragments which matched this configuration, theoretically placing the methyl group in position C2, C3 or C4 to see which fragments were expected after ethylation. We located a large peak containing the fragments 132, 190, 203 and $261 \mathrm{~m} / \mathrm{z}$, which were expected if a hexose was methylated at C3. Ultimately, this finding was verified by NMR spectroscopy including anomeric configuration (Fig. 3, Table 4), suggesting presence of $(1 \rightarrow 6)-\alpha-3-O-M e-G a l$ residues in most fractions. The present study is the first to report this uncommon monosaccharide in I. obliquus. There are some papers describing $(1 \rightarrow 6)-\alpha-3-O-\mathrm{Me}-\mathrm{Gal}$ from other fungi, such as species from the genera Phellinus and Pleurotus (Carbonero et al., 2008; Yang et al., 2007). With the presence of this residue in Inonotus as well, it seems that extensive 3-O-methylation of galactose is more common in polypore fungi than previously thought, although the biological role of such methylation is still largely unknown (Staudacher, 2012).

\subsection{Linkage analysis by methylation/GC-MS}

To reveal how the different monosaccharides were linked, the methylated alditol acetates of the monosaccharides were analyzed by GC-MS, as seen in Table 3. The results are presented as total mol\% of each sub-species based on the percentage of each monosaccharide initially found by GC analysis after methanolysis (Table 2). The most common glucose species in the water-extracted fractions were $(1 \rightarrow 3)$ linked Glc and $(1 \rightarrow 6)$-linked Glc. There were also substantial amounts of terminal (non-reducing) Glc (T-Glc) and ( $\rightarrow 3,6)$-linked Glc, indicating a highly branched structure of the polysaccharides. The presence of $(1 \rightarrow 2,3)$-linked Glc $(\sim 1 \%)$ and $(1 \rightarrow 2,3,6)$-linked Glc $(\sim 1 \%)$ suggest that glucose could be substituted at position C2 by arabinose, fucose, xylose and glucuronic acid, which were all present in minor amounts and were mostly of terminal origin. Galactose was the second most common monosaccharide, being almost exclusively $(1 \rightarrow$ 6)-linked, with small amounts of T-Gal and $(1 \rightarrow 2,6)$-linked Gal indicating that also galactose was substituted at $\mathrm{C} 2$. The percentage of the different galactose species found from GC-MS is a combination of native and methylated galactose, since these two types would appear identical after methylation of the samples, except for $(1 \rightarrow 3)$ - and $(1 \rightarrow 3,6)$-linked Gal which only could come from native galactose. Mannose was mostly $(1 \rightarrow 2)$ - and $(1 \rightarrow 3)$-linked, with some T-Man as well. The acidic fractions had substantial amounts of $(1 \rightarrow 4)$-linked GalA, which was virtually absent in the neutral fractions IOI-WN and IOE-WN.

The presence of $(1 \rightarrow 4)$-linked GalA, $(1 \rightarrow 2)$ - and $(1 \rightarrow 2,4)$-linked Rha, as well as some $(1 \rightarrow 3)$-linked Gal and T-Ara indicates that a part of the polysaccharides had a pectin-like structure (Yapo, 2011). It could be that some birch material was mixed with the fungal material prior to extraction and fractionation. If this were true, the traces should only be prominent in the fractions isolated from the interior part of the fungus, since this part is in direct contact with the birch. Since fractions isolated from both the interior and exterior parts contained the pectin-resembling linkages, it is likely that there exists some other explanation for 

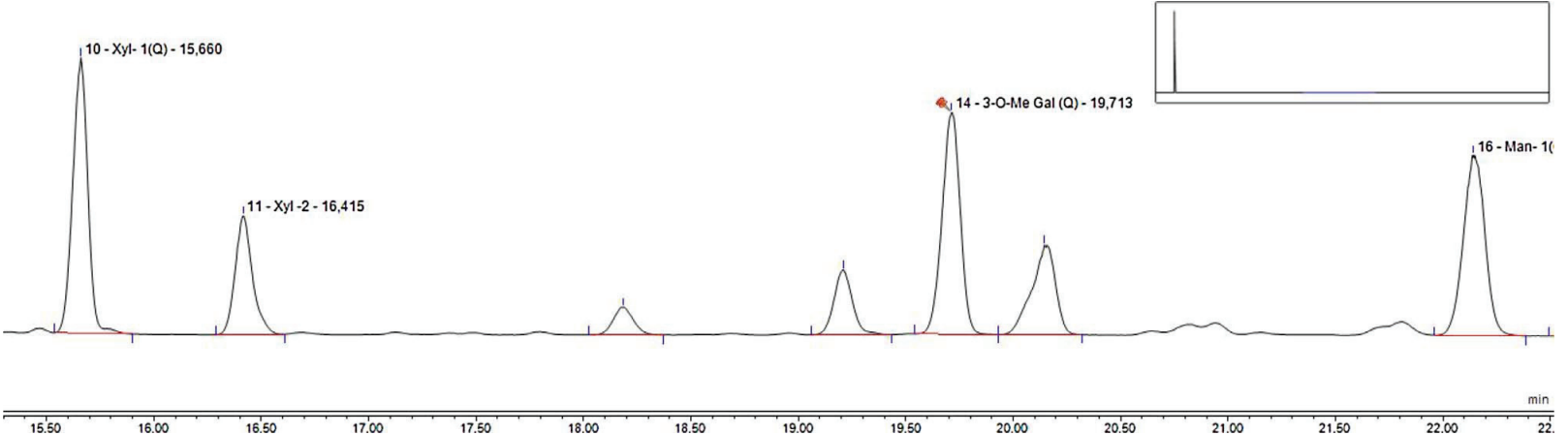

Fig. 2. The three chromatographic peaks (19-20.50 min) from 3-O-Me-Gal after methanolysis and GC analysis of IOE-WN, located between the peaks from xylose (15.5-16.5 min) and mannose (22 min).

the linkages observed.

\subsection{IR spectroscopy}

The polysaccharide fractions were analyzed by IR spectroscopy. There were several bands in the IR spectra that could be assigned to structural motifs from carbohydrates. There was a strong, but broad band at $3360 \mathrm{~cm}^{-1}$ coming from $-\mathrm{OH}$ groups, and several bands around $900-1050 \mathrm{~cm}^{-1}$ that probably came from $(\mathrm{C}-\mathrm{O}-\mathrm{C})$ in glycosidic linkages of various configurations. The bands from $\mathrm{C}-\mathrm{O}-\mathrm{C}$ will usually shift slightly depending on type of linkage $(\alpha, \beta, 1 \rightarrow 6$ vs $1 \rightarrow 3$ etc.) and it was therefore expected that this area was crowded with

Table 3

Glycosidic linkages (mol \%) in the polysaccharide fractions isolated from I. obliquus detected by GC-MS analysis of methylated alditol acetates.



(n.d. $=$ not detected; trace $=$ less than $1 \%$; $\mathrm{T}=$ terminal, non-reducing end). 


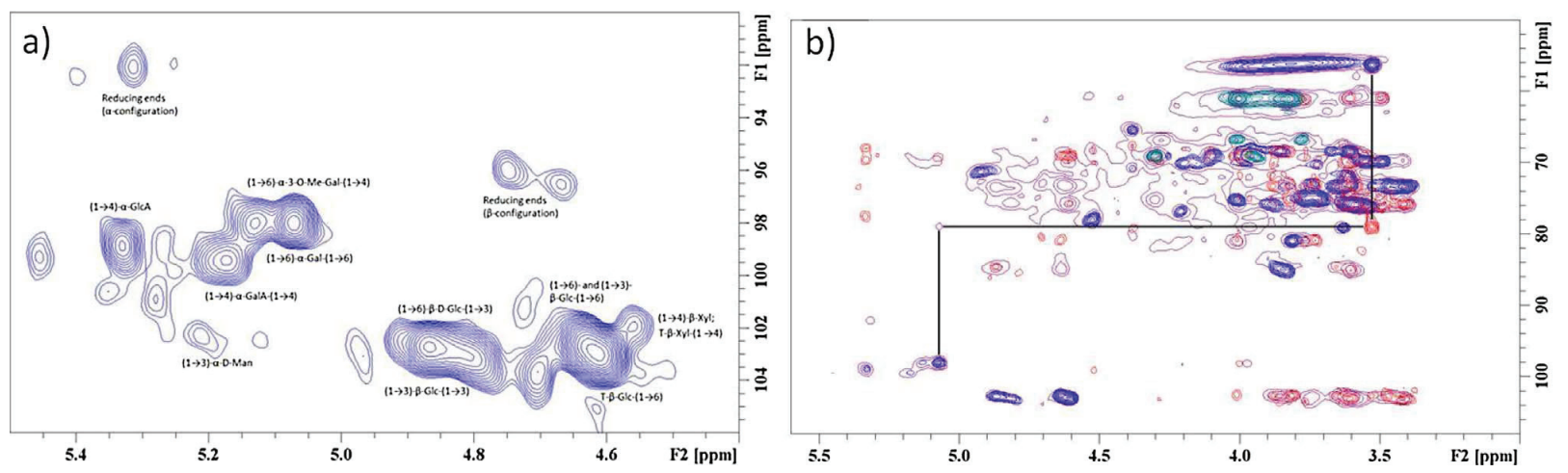

Fig. 3. (a) NMR analysis of fraction IOI-WAcF2, showing the anomeric region from the HSOC spectrum with assignments of the major signals in this region. Chemical shifts are expressed in ppm. (b) Overlay plot from IOI-WAcF2 of HSQC (blue)/HSQC-TOCSY (purple)/HMBC (red), showing the main carbohydrate region between $3-6 \mathrm{ppm} / 50-110 \mathrm{ppm}{ }^{1} \mathrm{H} /{ }^{13} \mathrm{C}$. This plot gives an idea of the complexity of the sample. The black line shows a direct link from the $O$-methyl signal at 3.53/56.02 ppm to a C3 signal from $\alpha$-Gal at $3.63 / 78.77 \mathrm{ppm}$, which could be traced back to the $\mathrm{C} 1$ signal at $5.07 / 97.72 \mathrm{ppm}$. This species was assigned as $(1 \rightarrow 6)-3-O-M e-\alpha-G a l$. (For interpretation of the references to colour in this figure legend, the reader is referred to the web version of this article.)

Table 4

Chemical shift values of the major signals found in the water-extracted polysaccharide fractions from $I$. obliquus. The values are expressed as ppm from each position in that species, from $\mathrm{C} 1 / \mathrm{H1}$ (1) to C6/H6 (6), and are based mainly on spectra from IOE-WN, IOI-WAc and IOI-WAcF1-F3. Some of the species could only be partially assigned due to overlaps and/or weak signal intensities.

\begin{tabular}{|c|c|c|c|c|c|c|c|}
\hline Type of monosaccharide w/linkage & 1 & 2 & 3 & 4 & 5 & 6 & 3-O-Me \\
\hline \multicolumn{8}{|l|}{$(1 \rightarrow 3)-\beta-$ Glc- $(1 \rightarrow 3)$} \\
\hline$\delta^{13} \mathrm{C}$ & 102.48 & 73.17 & 84.59 & 68.17 & 75.44 & 60.80 & \\
\hline$\delta^{1} \mathrm{H}$ & 4.87 & 3.65 & 3.85 & 3.61 & 3.6 & $3.83 / 4.03$ & \\
\hline \multicolumn{8}{|l|}{$1 \rightarrow 6)-\beta$-Glc- $(1 \rightarrow 3)$} \\
\hline$\delta^{13} \mathrm{C}$ & 102.92 & 73.35 & 75.42 & 69.62 & 74.59 & 68.85 & \\
\hline$\delta^{1} \mathrm{H}$ & 4.80 & 3.47 & 3.62 & 3.51 & 3.80 & $3.96 / 4.31$ & \\
\hline \multicolumn{8}{|l|}{$(1 \rightarrow 6)-\beta$-Glc- $(1 \rightarrow 6)$} \\
\hline$\delta^{13} \mathrm{C}$ & 102.72 & 73.05 & 75.62 & 69.52 & 74.82 & 68.85 & \\
\hline$\delta^{1} \mathrm{H}$ & 4.61 & 3.42 & 3.59 & 3.55 & 3.72 & $3.96 / 4.31$ & \\
\hline \multicolumn{8}{|l|}{$(1 \rightarrow 3)-\beta$-Glc- $(1 \rightarrow 6)$} \\
\hline$\delta^{13} \mathrm{C}$ & 102.40 & 72.58 & 84.79 & 68.11 & 75.44 & 60.80 & \\
\hline$\delta^{1} \mathrm{H}$ & 4.63 & 3.61 & 3.84 & 3.60 & 3.58 & $3.83 / 4.03$ & \\
\hline \multicolumn{8}{|l|}{ T- $\beta$-Glc- $(1 \rightarrow 6)$} \\
\hline$\delta^{13} \mathrm{C}$ & 102.72 & 73.05 & 75.62 & 69.52 & 74.82 & 60.80 & \\
\hline$\delta^{1} \mathrm{H}$ & 4.61 & 3.42 & 3.59 & 3.55 & 3.72 & $3.83 / 4.03$ & \\
\hline \multicolumn{8}{|l|}{$(1 \rightarrow 4)-\beta-X y l$} \\
\hline$\delta^{1} \mathrm{H}$ & 4.55 & 3.34 & 3.66 & 3.82 & $3.46 / 4.18$ & & \\
\hline \multicolumn{8}{|l|}{$\mathrm{T}-\beta-X y 1-(1 \rightarrow 4)$} \\
\hline$\delta^{13} \mathrm{C}$ & 101.69 & & & & 65.16 & & \\
\hline$\delta^{1} \mathrm{H}$ & 4.56 & 3.38 & & & $3.4 / 4.06$ & & \\
\hline \multicolumn{8}{|l|}{$(1 \rightarrow 6)-\alpha-$ Gal- $(1 \rightarrow 6)$} \\
\hline$\delta^{13} \mathrm{C}$ & 97.72 & 67.95 & 69.83 & 69.23 & 69.11 & 66.76 & \\
\hline$\delta^{1} \mathrm{H}$ & 5.08 & 3.93 & 3.97 & 4.11 & 4.26 & $3.78 / 4.01$ & \\
\hline \multicolumn{8}{|l|}{$(1 \rightarrow 6)-\alpha-3-O-M e-G a l-(1 \rightarrow 6)$} \\
\hline$\delta^{13} \mathrm{C}$ & 97.72 & 67.42 & 78.77 & 65.1 & 68.77 & 66.76 & 56.02 \\
\hline$\delta^{1} \mathrm{H}$ & 5.07 & 3.97 & 3.63 & 4.38 & 4.27 & $3.78 / 4.01$ & 3.53 \\
\hline \multicolumn{8}{|l|}{$(1 \rightarrow 4)-\alpha-$ GalA- $(1 \rightarrow 4)$} \\
\hline$\delta^{13} \mathrm{C}$ & 98.92 & 68.02 & 68.62 & 77.72 & 71.12 & 174.70 & \\
\hline$\delta^{1} \mathrm{H}$ & 5.17 & 3.85 & 4.09 & 4.52 & 4.85 & & \\
\hline \multicolumn{8}{|l|}{$(1 \rightarrow 3)-\alpha-$ Man } \\
\hline$\delta^{13} \mathrm{C}$ & 102.1 & 70.38 & 78.43 & & & 62.84 & \\
\hline$\delta^{1} \mathrm{H}$ & 5.21 & 3.86 & 4.12 & & & $3.82 / 3.98$ & \\
\hline \multicolumn{8}{|l|}{$(1 \rightarrow 4)-\alpha-G l c A$} \\
\hline$\delta^{1} \mathrm{H}$ & 5.34 & & 4.21 & & & & \\
\hline \multicolumn{8}{|l|}{$(1 \rightarrow 2)-\alpha-R h a$} \\
\hline$\delta^{13} \mathrm{C}$ & 102.3 & & & & & 16.93 & \\
\hline$\delta^{1} \mathrm{H}$ & 5.09 & 4.18 & & 3.5 & 3.9 & 1.33 & \\
\hline
\end{tabular}


signals, as observed in the spectra (Wiercigroch et al., 2017). In addition, a band with strong intensity could be observed at $1650 \mathrm{~cm}^{-1}$ in the acidic fractions, probably from a $\mathrm{C}=\mathrm{O}$ in a carboxylic group coming from the uronic acids.

\subsection{NMR spectroscopy}

The NMR data were interpreted by comparing and matching chemical shift values from the $1 \mathrm{D}$ spectra ${ }^{1} \mathrm{H}$ and ${ }^{13} \mathrm{C}$, and $2 \mathrm{D}$ spectra DQFCOSY, TOCSY, NOESY, HSQC, HSQC-TOSCY and HMBC. It was possible to deduce the monosaccharide structure for many of the major signals, including possible linkages. The polysaccharide fractions were analyzed separately, and differences between them were deduced by comparing the different NMR spectra and by comparison with GC- and GC-MS data. CASPER predictions and reference values from the literature were also used to assign the chemical shift values (Agrawal, 1992; Duus, Gotfredsen, \& Bock, 2000; Jansson, Stenutz, \& Widmalm, 2006; Lundborg \& Widmalm, 2011). In general, the signals belonging to the different monosaccharide species were assigned using the following procedure: First, an anomeric ${ }^{1} \mathrm{H}-{ }^{1} \mathrm{H}$ signal (H1) was located in a DQFCOSY spectrum. The $\mathrm{H} 1$ signal was then correlated with the $\mathrm{H} 2$ signal in the same spin system, and it was attempted to find further correlations to H3 and so on, using DQF-COSY and TOCSY. If the rest of the spin system could not be determined this way, HSQC-TOCSY, HMBC and NOESY spectra were used to find the last ${ }^{1} \mathrm{H}$ signals for that species and to look for long-range correlations and coupling to other monosaccharide species. HSQC and ${ }^{13} \mathrm{C}$ spectra were used continuously to assign the ${ }^{13} \mathrm{C}$ signals that correlated with the specific ${ }^{1} \mathrm{H}$ signals. The different samples were usually compared to each other using overlay plots, for example overlaying the HSQC spectra from IOE-WN and IOIWAcF1 to look for differences. If some signals could still not be assigned, either due to overlap with other signals or because of weak signal intensity, CASPER prediction was used to get an idea of the location (Jansson, Stenutz, \& Widmalm, 2006; Lundborg \& Widmalm, 2011). In general it was difficult to find evidence of linkages between the different monomers in the HMBC and NOESY spectra, such as direct linkages from Xyl to Glc, or from GalA to Gal. Since all fractions were highly heterogeneous, it means that either these linkage signals were too weak to observe by NMR analysis, or that the fractions consisted of several separate polymers which were diluted together during fractionation. However, the latter explanation seems unlikely. During column chromatography, we loaded sample onto the column step-wise, a small amount each time, and monitored the fractionation using the phenolsulfuric acid assay. The polysaccharides were eluted at the same point each time, and if the samples were to be a mix of "entangled" polymers, there would have been variation in elution times from experiment to experiment. It therefore seems more likely that the fractions contained heteropolysaccharides but that the linkages were too difficult to capture by NMR spectroscopy due to low relative abundance of each specific linkage signal. All water-soluble samples had presence of major peaks in the anomeric region, defined as having chemical shift values between 4.5-5.5 ppm for ${ }^{1} \mathrm{H}$ and $95-105$ ppm for ${ }^{13} \mathrm{C}$ (Fig. 3) (Duus, Gotfredsen, \& Bock, 2000). Typically, $\alpha$-anomeric signals will appear downfield of $\beta$-anomeric signals for ${ }^{1} \mathrm{H}$ chemical shifts, and opposite for ${ }^{13} \mathrm{C}$ chemical shifts. The chemical shift values of the major signals found are presented in Table 4. There was evidence of reducing ends present in some fractions (Figs. S9-S14). Although these signals correlated with the relative sizes of the fractions (lower $M_{w}$ means more reducing ends), the signals were stronger than they should be according to SEC-MALLS. For example, in IOI-WAcF2 the total signal strength of the reducing ends was about $6.7 \%$ compared to the anomeric signals, theoretically giving a $\mathrm{M}_{\mathrm{w}}$ of $\sim 2.5 \mathrm{kDa}$ instead of $14 \mathrm{kDa}$ as seen from SEC-MALLS (Table 1). One possible explanation for the observed effect is that reducing ends located near the surface would give stronger NMR signals than the monomers located inside the polymer, since they would be more flexible. This could be particularly true for the polysaccharides in this study, since the $R_{G}$ values suggest that the structures are very compact (Table 1). This could result in the reducing ends having T2 relaxation times more similar to oligo- or monosaccharides, and longer T2 relaxation times would in turn result in lower loss of magnetization during mixing times (Duus et al., 2000).

\subsection{1. $\beta$-glucose}

The HSQC spectra revealed the presence of two main signal clusters in the $\beta$-anomeric region located at $\left({ }^{1} \mathrm{H} /{ }^{13} \mathrm{C}\right) \sim 4.85 / 102.5 \mathrm{ppm}$ and $\sim 4.62 / 102.9 \mathrm{ppm}$ (Fig. 3). With the use of (DQF-COSY), TOCSY as well as HSQC-TOCSY spectra, the signals were suggested to come from $\beta$ glucose: $\beta$-Glc- $(1 \rightarrow 3)$-species at $\sim 4.85 / 103 \mathrm{ppm}$ and $\beta$-Glc- $(1 \rightarrow 6)$ species at $\sim 4.6 / 103 \mathrm{ppm}$ (Table 4 ). The peaks were too broad to contain just one signal, and the HSQC and ${ }^{13} \mathrm{C}$ spectra indicated presence of signals from several different anomeric carbons, probably variations of the different $\beta$-Glc species, such as $(1 \rightarrow 3-\beta-G l c-1 \rightarrow 3)$, $(1 \rightarrow 6-\beta-G l c-$ $1 \rightarrow 3)$, $(1 \rightarrow 3-\beta-G l c-1 \rightarrow 6)$, and $(1 \rightarrow 6-\beta-G l c-1 \rightarrow 6)$. Anomeric signals from even more complex species including $(1 \rightarrow 3,6)$-branching points, or with $\mathrm{C} 2$ in glucose additionally substituted by another monosaccharide as suggested from the linkage analysis data (Table 3) would also be located in the same anomeric signal clusters. Further, signals from terminal glucose would also be located here, as these could be defined as pure $\beta$-Glc- $(1 \rightarrow 3)$ or $\beta$-Glc- $(1 \rightarrow 6)$ species with no other substitutions. The two main clusters were defined on the basis of the assignment methods described above, and also because the two groups could be differentiated by the relatively large difference in shift values for C6 in the HSQC spectra, where the $(1 \rightarrow 6)-\beta$-Glc has a ${ }^{13} \mathrm{C}$ chemical shift downfield of its $(1 \rightarrow 3)-\beta$-Glc counterpart (Table 4$)$. In addition, the $\mathrm{C} 3$ signal from $(1 \rightarrow 3)-\beta$-Glc has a very characteristic chemical shift value, in our samples observed at $\sim 3.85 / 85 \mathrm{ppm}$ in the HSQC spectra, thus confirming that the signals come from glucose. It was attempted to find correlations between the various $\beta$-Glc species by comparing integrals in the HSQC spectra (which could not be done in 1D spectra due to overlaps). Although 2D integrals are less accurate and overlap also occurred in these spectra, some clues were found that could indicate a possible configuration. In IOE-WN, the HSQC integral from the anomeric $\beta$-Glc- $(1 \rightarrow 3)$ cluster was approximately the same size as the signal from $\mathrm{C} 3 / \mathrm{H} 3$ in $(1 \rightarrow 3)-\beta$-Glc. The signal from $\mathrm{C} 4 / \mathrm{H} 4$ in $(1 \rightarrow 3)$ $\beta$-Glc was about $25 \%$ smaller than the others, but a signal located at same ${ }^{13} \mathrm{C}$ ppm but $0.1{ }^{1} \mathrm{H}$ ppm downfield corresponded well with these $25 \%$ and according to CASPER this signal could come from $(1 \rightarrow 3,6)-\beta$ Glc- $(1 \rightarrow)$, suggesting several branching points in the polymer. The relative proportion of $(1 \rightarrow 3)$-linked and $(1 \rightarrow 6)$-linked $\beta$-Glc was approx. 1:1 for most samples according to GC-MS (Table 3), while the signal cluster in the HSQC spectra from $\beta$-Glc- $(1 \rightarrow 6)$-species was twice as big as the $\beta$-Glc- $(1 \rightarrow 3)$-cluster (Fig. 3). Methylation/GC-MS analysis does not give information on how terminal residues are linked but NMR does, and a possible explanation for the difference in signal strength could therefore be that most of the terminal Glc in our samples is $(1 \rightarrow 6)$-linked.

\subsection{2. $\beta$-xylose}

Some signals close to the two major peaks in the $\beta$-anomeric region were assigned to $(1 \rightarrow 4)$-linked $\beta$-xylose species (Fig. 3 , Table 4 ). $\beta$-Xyl has axial protons for $\mathrm{H} 1, \mathrm{H} 2, \mathrm{H} 3$ and $\mathrm{H} 4$, making it easier to detect than some other monosaccharides since the $\mathrm{H}, \mathrm{H}$ couplings will give strong signals which can be correlated through DQF-COSY and TOCSY. The anomeric ${ }^{1} \mathrm{H}$ signals could therefore be correlated throughout the molecule from $\mathrm{H} 1-\mathrm{H} 5$, but some of the ${ }^{13} \mathrm{C}$ signals could not be assigned due to overlaps (C3 and C4). In the HSQC spectra, opposite phase chemical shifts for $\mathrm{H} 5 \mathrm{a}$ and $\mathrm{H} 5 \mathrm{~b}$ were in accordance with CASPER prediction for $(1 \rightarrow 4)$ - $\beta$-Xyl and terminal $\beta$-Xyl $(1 \rightarrow 4)$. The $\mathrm{H}-\mathrm{H}$ split of $\mathrm{H} 5$ in xylose is quite large and characteristic compared to some other monosaccharides (Agrawal, 1992). There were some differences between the samples for the H5 signals. For example, IOE-WN had one clear ${ }^{1} \mathrm{H}-{ }^{1} \mathrm{H}$-split from $\mathrm{H} 5$ in $(1 \rightarrow 4)-\beta$-Xyl, while in IOI-WN there were 
two such splits differing by $0.05 \mathrm{ppm}$, suggesting more than one $(1 \rightarrow$ 4)- $\beta$-Xyl species in IOI-WN. This could mean that xylose chains of various lengths or xylose being in different local environments are present, giving rise to more signals. From GC-MS data it was found that the IOE-WN fraction had slightly more T-Xyl (4.7\%) than $(1 \rightarrow 4)$-Xyl (4.2\%), suggesting that xylose is substituting a main polysaccharide chain as either mono- or disaccharides with $(1 \rightarrow 4)$ linkages. The IOIWN fraction had slightly more $(1 \rightarrow 4)$-Xyl (3.8\%) than T-Xyl (3.1\%), suggesting that the xylose side chains would be of varying lengths, which would fit well with the NMR spectra observed. However, we could not observe any HMBC correlations from xylose to glucose or galactose, but this could also be due to the relative rarity of these linkages in the samples. As seen from Table 2, xylose is present in relatively high amounts in all fractions, also when galactose, mannose and galacturonic acid levels are low. It therefore seems likely that xylose is somehow linked to glucose within the polymers chain.

\subsection{3. $\alpha$-galactose and $\alpha-3-O-M e-G a l$}

All samples had presence of several strong signals in the $\alpha$-anomeric region (Fig. 3). The signal at 5.07/97.72 ppm was suggested to come from $(1 \rightarrow 6)-\alpha-3-O-M e-G a l$. Using DQF-COSY it was found that this signal actually correlated with two separate $\mathrm{H} 2$ signals, at 3.97 and $3.93 \mathrm{ppm}$. Using DQF-COSY and TOCSY the signals at $3.97 \mathrm{ppm}$ could be assigned through the spin system from H2-H6. The HMBC spectrum showed a correlation between the H3/C3 of this species to a methyl peak at 3.53/56.02 ppm, and as such this species was designated $(1 \rightarrow$ $6)-\alpha-3-O-M e-G a l$. The other signal in the same anomeric peak was assigned to $(1 \rightarrow 6)-\alpha$-Gal. According to CASPER prediction, the anomeric ${ }^{13} \mathrm{C}$ chemical shift values from these two species should actually be separated by $2 \mathrm{ppm}$. However, since the signal cluster ranged from $\sim 96.80-99 \mathrm{ppm}$, and others also have reported the same findings as us, it seems logical that the two signals could be located in the same area (Smiderle et al., 2008). This would also explain the relatively strong intensity of the anomeric peak, which had equal signal intensity as the $\beta$-Glc- $(1 \rightarrow 6)$ signal cluster. In both species the rest of the ${ }^{13} \mathrm{C}$ signals could be assigned using HSQC and HMBC. The ${ }^{13} \mathrm{C}$ signal from C6 could not be differentiated between the two species, and had a relatively downfield shift value compared to the expected value for galactose (Agrawal, 1992). This was probably due to the substitution at C6 with another $\mathrm{Gal}$ residue, which was further strengthened by finding a strong correlation from $\mathrm{C} 1$ to $\mathrm{C} 6$ in $\mathrm{HMBC}$ as well, suggesting that a part of the polymer chain has a $(1 \rightarrow 6)-\alpha-G a l-(1 \rightarrow 6)$ motif with about every second or third residue being 3-O-methylated.

\subsection{4. $\alpha$-GalA}

In the acidic fractions only, a signal at 5.17/98.92 ppm with strong intensity was found in the $\alpha$-anomeric region (Fig. 3a, Figs. S9-S14). The signal was suggested to come from $(1 \rightarrow 4)-\alpha$-GalA since this species should be present in high amounts in acidic fractions and absent from the neutral fractions according to GC-MS data (Table 3). The signal could be correlated throughout the ${ }^{1} \mathrm{H}$ spin system from $\mathrm{H} 1-\mathrm{H} 5$ and it was also possible to assign the ${ }^{13} \mathrm{C}$ signals to each ${ }^{1} \mathrm{H}$ signal (Table 4). According to CASPER prediction, GalA was expected to have ${ }^{1} \mathrm{H}$ chemical shifts values for $\mathrm{H} 4$ and $\mathrm{H} 5$ close to the $\beta$-anomeric region but with highly different ${ }^{13} \mathrm{C}$ values, and this seemed to be the case in our samples as well, with $\mathrm{H} 4$ at $4.52 \mathrm{ppm}$ and $\mathrm{H} 5$ at $4.85 \mathrm{ppm}$ (Agrawal, 1992). These two signals could be used to distinguish GalA from GlcA, since GlcA would have ${ }^{1} \mathrm{H}$ shift values at much lower ppm according to CASPER. In the ${ }^{13} \mathrm{C}$ and $\mathrm{HMBC}$ spectra there was a signal at $174.70 \mathrm{ppm}$ which indicated presence of a $\mathrm{C} 6$ carboxyl group coming from an uronic acid. Further, in several HMBC spectra we could correlate C4 and H1 and $\mathrm{H} 4$ and $\mathrm{C} 1$ to each other by HMBC, indicating that this species was a long chain of $(1 \rightarrow 4)-\alpha$-GalA- $(1 \rightarrow 4)$. No linkage correlations between GalA and other species could be observed, but this could be due to a very low relative abundance of monomers linking the GalA chain to the rest of the polymer.

\subsubsection{Other $\alpha$-linked species}

In most fractions there were also several minor signals that could not be completely assigned to specific species due to signal overlap and the relative weakness of the signals (Fig. 3a). GC and GC-MS data indicated that glucuronic acid, rhamnose and mannose were present in various amounts in all samples (Table 3) and several of the signals in the $\alpha$-anomeric region probably came from these monosaccharides. As for GlcA it was difficult to locate most signals, probably because of the low abundance in all samples (Table 4). According to CASPER prediction, $(1 \rightarrow 4)-\alpha$-GlcA is expected to have anomeric ${ }^{1} \mathrm{H}$ chemical shift values downfield of GalA, and the anomeric signal at 5.33/98.62 was consistently present in the acidic fractions where $(1 \rightarrow 4)$-GlcA was present. Although clear correlations to other signals could not be seen using DQF-COSY, the anomeric signal could be correlated to a ${ }^{1} \mathrm{H}$ signal at $4.21 \mathrm{ppm}$ using the TOCSY spectrum, which could be from $\mathrm{H} 3$ in the same spin system, and this also was in relative accordance with CASPER prediction. Other signals from $(1 \rightarrow 4)$-GlcA could not be observed due to unknown reasons, thus the signal assignments remains unclear. GC-MS data also indicated the presence of $(1 \rightarrow 2)$ - and $(1 \rightarrow 3)$-linked mannose, and mannose is usually in the $\alpha$-configuration in fungi (Arana et al., 2009). The HSQC signal at 5.21/102.27 ppm could come from $(1 \rightarrow 3)$-linked $\alpha$-Man (Fig. 3). However, this signal could not be correlated further to the $\mathrm{H} 2$ signal, which could be due to the fact that mannose can be quite difficult to deduce by NMR spectroscopy, as the equatorial $\mathrm{H} 2$ gives weak couplings to $\mathrm{H} 1$ and $\mathrm{H} 3$. This means that high amounts of each mannose species probably would be required for sufficient signal intensities to be produced. The absence of clear correlations is in this sense in accordance with GS-MS data which suggested that only small amounts of each mannose species were present in the samples (Table 3). Additionally, it was only in IOI-WAcF2 - the fraction with the highest amount of $(1 \rightarrow 3)$ - $\alpha$-Man - that the anomeric signal could be located clearly, with some traces in the IOI-WAc sample as well. There was also a slight indication of a correlation between ${ }^{1} \mathrm{H}$ signals at 4.155 and $5.369 \mathrm{ppm}$ in NOESY, which could come from mannose (Fig. S15). However, this could not be found in HSQC-TOCSY or other spectra, which makes it possible that it could come from across the glycosidic linkage and not from the same monomer.

Rhamnose was thought to be in $\alpha$-configuration due to the presence of several non-assigned anomeric signals in the $\alpha$-anomeric region. Using spectra from IOI-WAcF3, a signal was located at 1.33/16.93 ppm in HSQC which likely was from a methyl in a 6-deoxy sugar. In DQFCOSY, this signal had correlations to another ${ }^{1} \mathrm{H}$ signal at $\sim 3.90 \mathrm{ppm}$, which could be the H5 signal from $\alpha$-rhamnose (Agrawal, 1992). This signal could be correlated to signals at $\sim 3.5 \mathrm{ppm}$ and $4.18 \mathrm{ppm}$ using TOCSY, and although the peaks were quite broad this suggests the presence of at least one $\alpha$-Rha species in the samples, probably $(1 \rightarrow 2)$ $\alpha$-Rha (Figs. S16 and S17).

\subsubsection{IOI-AI}

The alkali-extracted fraction IOI-A1 was also subjected to NMR analysis. However, since the fraction was dissolvable only in alkali and neither $\mathrm{H}_{2} \mathrm{O}$ or dimethyl sulfoxide (DMSO), the sample was dissolved in $0.1 \mathrm{M} \mathrm{NaOH}$ in $\mathrm{D}_{2} \mathrm{O}$ which led to some results not consistent with the other samples. The HSQC spectrum revealed presence of some strong signals coming from $\beta$-Glc species (Fig. S18). Some of the signals could be assigned with a high degree of certainty, such as two $(1 \rightarrow 3)-\beta-G l c$ signals at 3.79/87.7 ppm (from H3/C3) and 3.83/4.02/61.1 ppm (from $\mathrm{H} 6 \mathrm{a} / \mathrm{H} 6 \mathrm{~b} / \mathrm{C} 6)$. Other signals seemed to come from $(1 \rightarrow 6)$-linked species. Some of the signals had shifted notably compared to the other samples, possibly due to the difference in $\mathrm{pH}$. In addition, the large size of the IOI-A1 fraction ( $>450 \mathrm{kDa}$ ) and the presence of $\mathrm{Na}^{+}$in the sample could give weaker signals than expected, especially for the backbone of the polymer which is primarily made up of $(1 \rightarrow 3)$-linked $\beta$-Glc, whereas the side-chains of higher mobility would be less affected.

The GC-MS data suggested that IOI-A1 contained mainly $(1 \rightarrow 3)$ - 
Glc (57.3\%), with presence of $(1 \rightarrow 6)$-Glc $(3.3 \%),(1 \rightarrow 3,6)-$-Glc $(5.6 \%)$ and T-Glc (8\%) (Table 3). The strongest signals seen in ${ }^{13} \mathrm{C}$ (Fig. S31) and HSQC spectra of IOI-A1 came from what seemed to be either $(1 \rightarrow$ $6)$-linked $\beta$-Glc or $(1 \rightarrow 3,6)-\beta$-Glc species, and it therefore seems likely that the terminal $\beta$-Glc would be $(1 \rightarrow 6)$-linked, thus accounting for some of the signals seen in the NMR spectra. This would give a polymer with a backbone consisting of $(1 \rightarrow 3)$-linked $\beta$-Glc with short side chains of $(1 \rightarrow 6)$-linked $\beta$-Glc, possibly substituted with $\beta$-Xyl in between.

\subsection{Periodate oxidation and Smith degradation}

IOE-WN was subjected to periodate oxidation and Smith degradation to find clues about the relationship between homologous stretches in the polymer chain and linkages. Since the $(1 \rightarrow 3)$-linked sugars should not be hydrolyzed by the weak acid utilized after periodate oxidation ( $0.05 \mathrm{M}$ TFA), it would be expected that some oligosaccharides were produced if stretches of $(1 \rightarrow 3)$-linked $\beta$-Glc existed (Kamerling \& Gerwig, 2007). Fractionation of the degraded sample gave three main fractions which were analyzed by GC. While the two first fractions consisted of monomers (3-O-Me-Gal and Glc, respectively), the last fraction had a retention time in the middle of the maltotetraose and maltohexaose standards, and contained only glucose. This indicated that there were stretches of about $4-6(1 \rightarrow 3)$-linked glucose monomers before a change in the polymer chain occurs. Given the relatively large proportion of $(1 \rightarrow 6)$ linked Glc and Gal in the samples, it could be that the main motif of the polysaccharides is $(1 \rightarrow$ 3)-linked $\beta$-Glc five monomers long with $(1 \rightarrow 6)$-linked "kinks" in between with either Glc or Gal residues. There are some reports of similar structures in the literature, such as galactoglucans from the fungus Lentinus fusipes (Manna et al., 2017) and from the bacteria Rhizobium meliloti (Zevenhuizen, 1997). In addition, alternating $(1 \rightarrow 4) /(1 \rightarrow 3)$ linkages of glucose is the main motif in oat and barley $\beta$-glucans (Brennan \& Cleary, 2005), suggesting that alternating linkages in the main chain of polysaccharides could exist in other species such as fungi as well.

\subsection{Nitric oxide (NO) assay}

The polysaccharide fractions were screened for their ability to induce nitric oxide (NO) production in the murine dendritic cell line D2SC/1 and the murine macrophage cell line J774A1. NO regulates many processes in the immune system and is an important molecule in human physiology (Hickok \& Thomas, 2010), and high NO production by macrophages and dendritic cells can be considered a marker for a pro-inflammatory phenotype (Curren Smith, 2015). To investigate the immunomodulating effect of I. obliquus polysaccharides, cells were incubated with polysaccharide fractions with or without IFN- $\gamma$ for $24 \mathrm{~h}$. IFN- $\gamma$ has been shown to have a synergistic effect with TLR ligands on NO production in immune cells (Qiao et al., 2013; Totemeyer et al., 2006). The results are presented in Fig. 4. Although the fractions failed to activate NO production when used alone, most fractions demonstrated potent activity with IFN- $\gamma$ at the highest concentration $(100 \mu \mathrm{g} /$ $\mathrm{ml}$ ), indicating a synergistic effect which also was observed using the positive control Pam3CSK4. The neutral fraction IOE-WN gave a clear dose-response relationship, and was therefore considered the most promising fraction in this assay. IOE-WAc initially seemed promising; however it was found that this fraction contained LPS equivalent to $160 \mathrm{ng} / \mathrm{ml}$ at $100 \mu \mathrm{g} / \mathrm{ml}(0.16 \%)$. The acidic fractions from the interior part contained polyphenolic traces which could influence the results. In preliminary experiments a phenolic fraction from I. obliquus had a repressive effect on NO production in J774.A1 cells pretreated with LPS and IFN- $\gamma$ (data not shown), whereas others have found the same effect using polyphenolic extracts from various plants and fungi (Diaz, Jeong, Lee, Khoo, \& Koyyalamudi, 2012). The absence of a clear dose-response relationship in the acidic fractions from the interior part (containing
4.2-9.7\% polyphenols) could therefore be due to the presence of polyphenols although it would not explain why the same fractions induced potent NO production at $100 \mu \mathrm{g} / \mathrm{ml}$. The alkali-extracted IOI-A1 was not able to induce NO production in a dose-dependent manner, but demonstrated some activation even at the lowest concentration $(1 \mu \mathrm{g}$ ) $\mathrm{ml}$ with IFN- $\gamma$ ) in the D2SC/1 cell line.

\subsection{Structure-function relationship}

A clear structure-function relationship of the polysaccharides and their immunomodulating effects could not be established using the NO assay alone, but some suggestions were made. Since the neutral fractions seemed more active than the acidic ones, the GalA residues were probably not the most important for immune cell activation. Further, there was no obvious correlation between the amount of galactose and activity, since IOI-WAcF1 ( $\sim 20 \%$ Gal/3-O-Me-Gal) and IOI-WAcF3 ( $\sim 9 \% \mathrm{Gal} / 3-\mathrm{O}-\mathrm{Me}-\mathrm{Gal}$ ) gave similar results. Since the alkaline fraction IOI-A1 was not very active, it seems logical to conclude that soluble polysaccharides were better immune cell activators than particulate ones in this assay, in contrast with previous reports (Goodridge et al., 2011). However, the relative inactivity of IOI-A1 could also be due to its large size rather than solubility, or some other factors like the presence of xylose (14\%) in this fraction. The two neutral fractions IOI-WN and IOE-WN were very similar in terms of monosaccharide composition and types of linkages found, but IOE-WN seemed more promising in the NO assay. IOE-WN was slightly larger, having an $\mathrm{M}_{\mathrm{w}}$ of $73 \mathrm{kDa}$ vs. $60 \mathrm{kDa}$ for IOI-WN. In addition, IOE-WN had a narrower weight-distribution according to SEC-MALLS which means a more homogenous polysaccharide fraction, and this could also explain some of the difference in activity. It seems likely that the $(1 \rightarrow 3) /(1 \rightarrow 6)$ - $\beta$-Glc motif is, at least in part, responsible for the main effects observed in the experiments, but other factors such as three-dimensional shape could also be important. Fungal $\beta$-glucans of various kinds have previously been shown to be potent immune activators, and many different immune cell receptors have been associated with the recognition of such fungal structures (Erwig \& Gow, 2016). Dectin-1 has for example been established as the main receptor for particulate $(1 \rightarrow 3)$ - $\beta$-glucans (Goodridge et al., 2011), but it could very well be that other receptors are involved in the recognition of soluble $\beta$-glucans such as the ones from $I$. obliquus described herein. One study found an increased production of TNF- $\alpha$ by RAW264.7 cells after treatment with a polysaccharide extract from $I$. obliquus, and this effect was sugggested to be via Toll-like receptor 2 (TLR2) interaction and not Dectin-1 or TLR-4 (Won et al., 2011). Although the effects in the mentioned study were measured indirectly, and the experiment was conducted using a crude polysaccharide extract obtained by ethanol precipiation only (probably containing substantial amount of melanin as well), the results encourage further testing of purified $I$. obliquus polysaccharides in similar assays.

\section{Conclusion}

To our knowledge, this study is the first comprehensive characterization of polysaccharides from the medicinal fungus I. obliquus. Waterextracted neutral and acidic polysaccharide fractions as well as an alkali-extracted polysaccharide fraction were isolated from the interior (IOI) and exterior (IOE) parts of I. obliquus sclerotia and characterized by chromatographic and spectroscopic methods. No major structural differences between fractions from IOI and IOE were observed. The neutral polysaccharides IOI-WN $(60 \mathrm{kDa})$ and IOE-WN $(73 \mathrm{kDa})$ were heterogeneous and branched and consisted of a $(1 \rightarrow 3)$-linked $\beta$-Glc backbone with $(1 \rightarrow 6)$-linked kinks in the chain at approximately every fifth residue, with branches of $(1 \rightarrow 6)$-linked $\beta$-Glc in addition to substantial amounts of $(1 \rightarrow 6)$-linked $\alpha$-Gal with 3 -O-methylation at about every third Gal residue. The acidic polysaccharide fractions IOIWAc, IOI-WAcF1, F2 and F3 and IOE-WAc (10-31 kDa) showed similar structural motifs as the neutral ones, differing mainly by the presence of 


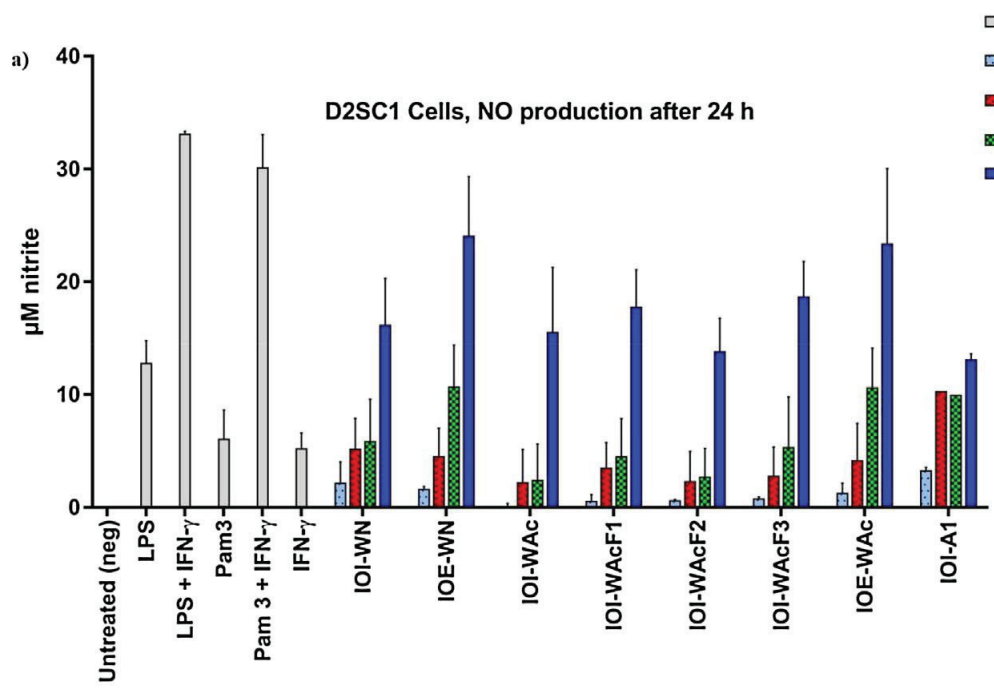

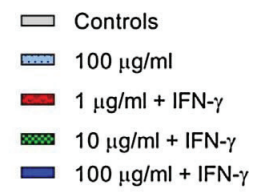

Fig. 4. NO production expressed as $\mu \mathrm{M}$ nitrite in the supernatant from D2SC/1 cells (a) and J774A.1 cells (b) after treatment with $I$. obliquus polysaccharide fractions with/without IFN- $\gamma$ for $24 \mathrm{~h}$. LPS $(1 \mu \mathrm{g} / \mathrm{ml})$ Pam3CSK4 $(2 \mu \mathrm{g} / \mathrm{ml})$ and IFN- $\gamma(20 \mathrm{ng} / \mathrm{ml})$ were used as positive controls alone and in combination. The untreated negative control had nitrite values ranging from 0.2 to $1 \mu \mathrm{M}$ but this was set to zero for the presentation of the results.

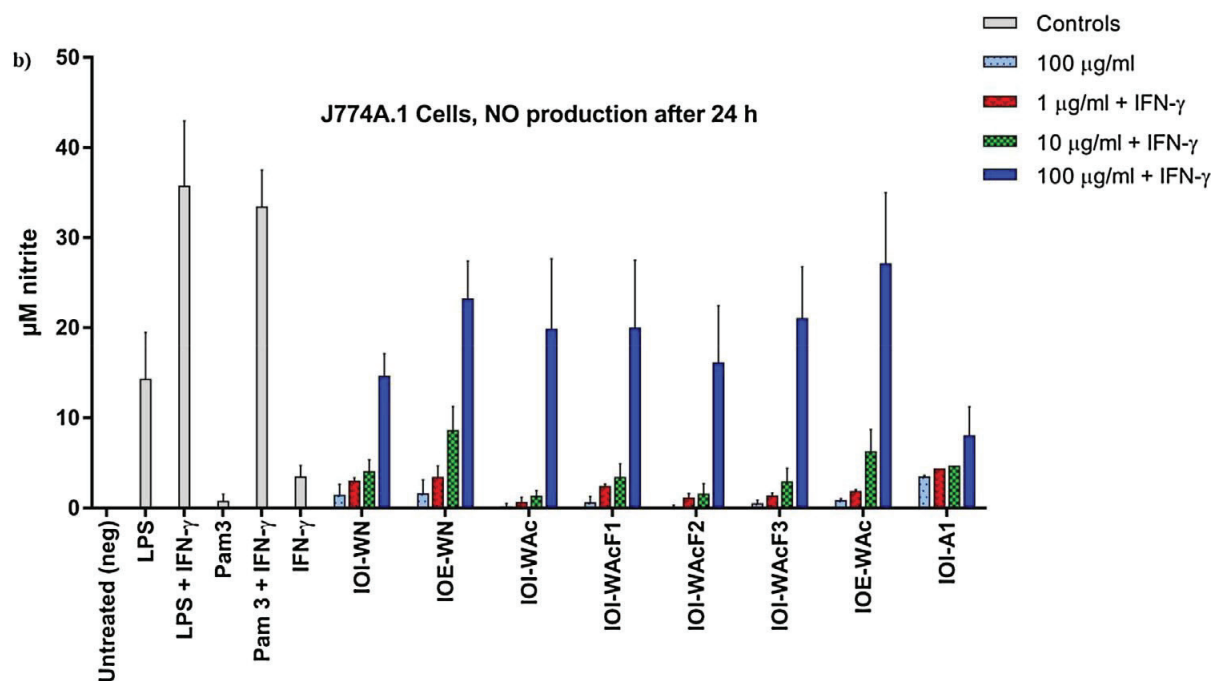

$(1 \rightarrow 4)$-linked $\alpha$-GalA and $\alpha$-GlcA. $\beta$-Xyl, $\alpha$-Man and $\alpha$-Rha were present in varying amounts in all fractions. The alkaline polysaccharide fraction IOI-A1 ( $>450 \mathrm{kDa}$ ) consisted mainly of $(1 \rightarrow 3)$ - and $(1 \rightarrow 6)$ linked $\beta$-Glc and $(1 \rightarrow 4)$-linked $\beta$-Xyl. GC-MS data from all fractions indicated that the polymer branching points were mainly found on $\beta$ Glc residues, thus these residues possibly connect the different parts of the polymers. However, such linkages could not be directly observed by NMR, and it should be attempted to clarify this in future studies. Several of the polysaccharide fractions showed promising results as immunomodulators as seen from the synergistic effect with IFN- $\gamma$ on NO production in the murine cell lines J774.A1 and D2SC/1. The neutral polysaccharide fraction IOE-WN showed the most potent effect in the assay, increasing NO production at $10 \mu \mathrm{g} / \mathrm{ml}$. It seems likely that the $(1 \rightarrow 3) /(1 \rightarrow 6)-\beta$-Glc motif within the polymer is important for bioactivity, but other factors such as three-dimensional shape could be equally important for the immunomodulating effect. The results presented herein encourage further exploration of the fine-structure, the structure-function relationship and bioactivity of polysaccharides from I. obliquus.

\section{Acknowledgements}

The authors would like to thank professor emerita Berit Smestad Paulsen for valuable discussions and interpretation of the results in general, Hoai Thi Nguyen Aas for help with the LPS determination assay, and Ellen Hanne Cohen for help with the Dionex column for the periodate oxidation/Smith degradation experiment. The work was partially funded by Novo Nordisk Foundation: Biotechnology-based synthesis and production research, program grant 5371. The $800 \mathrm{Mhz}$ NMR spectra for most samples were recorded on the NMR Center DTU, supported by the Villum Foundation. The work was partly supported by the Research Council of Norway through the Norwegian NMR platform, NNP (226244/F50). Finally the authors would like to thank the Norwegian PhD School of Pharmacy for funding a 3-week research stay at DTU for the NMR experiments.

\section{Appendix A. Supplementary data}

Supplementary material related to this article can be found, in the online version, at doi:https://doi.org/10.1016/j.carbpol.2017.12.041

\section{References}

Agrawal, K. A. (1992). NMR spectroscopy in the structural elucidation of oligosaccharides and glycosides. Phytochemistry, 31(10), 3307-3330.

Arana, D. M., Prieto, D., Roman, E., Nombela, C., Alonso-Monge, R., \& Pla, J. (2009). The role of the cell wall in fungal pathogenesis. Microbial Biotechnology, 2(3), 308-320. Austarheim, I., Christensen, B. E., Hegna, I. K., Petersen, B. O., Duus, J. O., Bye, R., ... Paulsen, B. S. (2012). Chemical and biological characterization of pectin-like polysaccharides from the bark of the Malian medicinal tree cola cordifolia. Carbohydrate 
Polymers, 89(1), 259-268.

Bouchard, A., Hofland, G. W., \& Witkamp, G. J. (2007). Properties of sugar, polyol, an polysaccharide water-ethanol solutions. Journal of Chemical \& Engineering Data, 52, $1838-1842$.

Bradford, M. M. (1976). A rapid and sensitive method for the quantitation of microgram quantities of protein utilizing the principle of protein-dye binding. Analytical Biochemistry, 72, 248-254.

Brennan, C. S., \& Cleary, L. J. (2005). The potential use of cereal $(1 \rightarrow 3,1 \rightarrow 4)-\beta$-d-glucans as functional food ingredients. Journal of Cereal Science, 42(1), 1-13.

Carbonero, E. R., Gracher, A. H., Rosa, M. C., Torri, G., Sassaki, G. L., Gorin, P. A., .. Iacomini, M. (2008). Unusual partially 3-O-methylated alpha-galactan from mushrooms of the genus pleurotus. Phytochemistry, 69(1), 252-257.

Cha, J. Y., Lee, S. Y., Lee, S. Y., \& Chun, K. W. (2011). Basidiocarp formation by Inonotus obliquus on a living paper birch tree. Forest Pathology, 41(2), 163-164.

Chambers, R. E., \& Clamp, J. R. (1971). An assessment of methanolysis and other factors used in the analysis of carbohydrate-containing materials. Biochemical Journal, 125, 1009-1018.

Chen, Y., Huang, Y., Cui, Z., \& Liu, J. (2015). Purification, characterization and biological activity of a novel polysaccharide from Inonotus obliquus. International Journal of Biological Macromolecules, 79, 587-594.

Curren Smith, E. W. (2015). Macrophage polarization and its role in cancer. Journal of Clinical and Cellular Immunology, 06(04).

de Santana-Filho, A. P., Noleto, G. R., Gorin, P. A. J., de Souza, L. M., Iacomini, M., \& Sassaki, G. L. (2012). GC-MS detection and quantification of lipopolysaccharides in polysaccharides through 3-O-acetyl fatty acid methyl esters. Carbohydrate Polymers, $87(4), 2730-2734$

Diaz, P., Jeong, S. C., Lee, S., Khoo, C., \& Koyyalamudi, S. R. (2012). Antioxidant and antiinflammatory activities of selected medicinal plants and fungi containing phenolic and flavonoid compounds. Chinese Medicine, $7(26)$

Du, B., Lin, C., Bian, Z., \& Xu, B. (2015). An insight into anti-inflammatory effects of fungal beta-glucans. Trends in Food Science \& Technology, 41(1), 49-59.

Dubois, M., Gilles, K. A., Hamilton, J. K., Rebers, P. A., \& Smith, F. (1956). Colorimetric method for determination of sugars and related substances. Analytical Chemistry, 28, 350-356.

Duus, J..Ø., Gotfredsen, C. H., \& Bock, K. (2000). Carbohydrate structural determination by NMR spectroscopy: Modern methods and limitations. Chemical Reviews, 100, 4589-4614.

Eisenman, H. C., \& Casadevall, A. (2012). Synthesis and assembly of fungal melanin. Applied Microbiology and Biotechnology, 93(3), 931-940.

Erwig, L. P., \& Gow, N. A. (2016). Interactions of fungal pathogens with phagocytes. Nature Reviews Microbiology, 14(3), 163-176.

Fan, L., Ding, S., Ai, L., \& Deng, K. (2012). Antitumor and immunomodulatory activity of water-soluble polysaccharide from Inonotus obliquus. Carbohydrate Polymers, 90(2), 870-874.

Fraser, I. P., Stuart, L., \& Ezekowitz, R. A. (2004). TLR-independent pattern recognition receptors and anti-inflammatory mechanisms. Journal of Endotoxin Research, 10(2), $120-124$

Glamoclija, J., Ciric, A., Nikolic, M., Fernandes, A., Barros, L., Calhelha, R. C., ... van Griensven, L. J. (2015). Chemical characterization and biological activity of Chaga (Inonotus obliquus), a medicinal "mushroom". Journal of Ethnopharmacology, 162, 323-332.

Goodridge, H. S., Reyes, C. N., Becker, C. A., Katsumoto, T. R., Ma, J., Wolf, A. J., ... Underhill, D. M. (2011). Activation of the innate immune receptor Dectin-1 upon formation of a 'phagocytic synapse'. Nature, 472(7344), 471-475.

Hickok, J. R., \& Thomas, D. D. (2010). Nitric oxide and cancer therapy: The emperor has NO clothes. Current Pharmaceutical Design, 16(4), 381-391.

Hu, Y., Sheng, Y., Yu, M., Li, K., Ren, G., Xu, X., ... Qu, J. (2016). Antioxidant activity of Inonotus obliquus polysaccharide and its amelioration for chronic pancreatitis in mice. International Journal of Biological Macromolecules, 87, 348-356.

Huang, S. Q., Li, J. W., Wang, Z., Pan, H. X., Chen, J. X., \& Ning, Z. X. (2010) Optimization of alkaline extraction of polysaccharides from Ganoderma lucidum and their effect on immune function in mice. Molecules, 15(5), 3694-3708.

Hwang, B. S., Lee, I. K., \& Yun, B. S. (2016). Phenolic compounds from the fungus Inonotus obliquus and their antioxidant properties. The Journal of Antibiotics, 69(2), $108-110$.

Ciucanu, I., \& Kerek, F. (1984). A simple and rapid method for the permethylation of carbohydrates. Carbohydrate Research, 131, 209-217.

Ina, K., Kataoka, T., \& Ando, T. (2013). The use of lentinan for treating gastric cancer. Anti-Cancer Agents in Medicinal Chemistry, 13, 681-688.

Jansson, P. E., Stenutz, R., \& Widmalm, G. (2006). Sequence determination of oligosaccharides and regular polysaccharides using NMR spectroscopy and a novel webbased version of the computer program CASPER. Carbohydrate Research, 341(8), 1003-1010.

Kamerling, J. P., \& Gerwig, G. J. (2007). Strategies for the structural analysis of carbohydrates. Elsevier Ltd.

Kapoor, S. (2014). Lentinan: Clinical benefit in the management of systemic malignancies. Surgery Today, 44(7), 1389.

Kim, Y. O., Han, S. B., Lee, H. W., Ahn, H. J., Yoon, Y. D., Jung, J. K., ... Shin, C. S. (2005) Immuno-stimulating effect of the endo-polysaccharide produced by submerged culture of Inonotus obliquus. Life Sciences, 77(19), 2438-2456.

Kim, Y. O., Park, H. W., Kim, J. H., Lee, J. Y., Moon, S. H., \& Shin, C. S. (2006). Anticancer effect and structural characterization of endo-polysaccharide from cultivated mycelia of Inonotus obliquus. Life Sciences, 79(1), 72-80.

Kukulyanskaya, T. A., Kurchenko, N. V., Kurchenko, V. P., \& Babitskaya, V. G. (2002) Physicochemical properties of melanins produced by the sterile form of Inonotus obliquus ("Chagi") in natural and cultivated fungus. Applied Biochemistry and
Microbiology, 38(1), 58-61.

Lee, K. R., Lee, J. S., Song, J. E., Ha, S. J., \& Hong, E. K. (2014). Inonotus obliquus-derive polysaccharide inhibits the migration and invasion of human non-small cell lung carcinoma cells via suppression of MMP-2 and MMP-9. International Journal of Oncology, 45(6), 2533-2540.

Lieder, R., Petersen, P. H., \& Sigurjonsson, O. E. (2013). Endotoxins-the invisible companion in biomaterials research. Tissue Engineering Part B: Reviews, 19(5), 391-402.

Liu, J., Willför, S., \& Xu, C. (2015). A review of bioactive plant polysaccharides: Biologica activities, functionalization, and biomedical applications. Bioactive Carbohydrates and Dietary Fibre, 5(1), 31-61.

Lu, C. C., Hsu, Y. J., Chang, C. J., Lin, C. S., Martel, J., Ojcius, D. M., ... Young, J. D. (2016). Immunomodulatory properties of medicinal mushrooms: Differential effects of water and ethanol extracts on NK cell-mediated cytotoxicity. Innate Immunity, 22(7), 522-533.

Lundborg, M., \& Widmalm, G. (2011). Structural analysis of glycans by NMR chemical shift prediction. Analytical Chemistry, 83(5), 1514-1517.

Ma, L., Chen, H., Dong, P., \& Lu, X. (2013). Anti-inflammatory and anticancer activities of extracts and compounds from the mushroom Inonotus obliquus. Food Chemistry, 139(1-4), 503-508.

Manna, D. K., Maity, P., Nandi, A. K., Pattanayak, M., Panda, B. C., Mandal, A. K. ... Islam, S. S. (2017). Structural elucidation and immunostimulating property of a nove polysaccharide extracted from an edible mushroom Lentinus fusipes. Carbohydrate Polymers, 157, 1657-1665.

Min-Woong, L., Hur, Hyeon, Kwang-Choon, C., Tae-Soo, L., Kang-Hyeon, K., \& Jankovsky, L. (2008). Introduction to distribution and ecology of sterile conks of Inonotus obliquus. Mycobiology, 36(4), 199-202.

Nyman, A. A., Aachmann, F. L., Rise, F., Ballance, S., \& Samuelsen, A. B. (2016). Structural characterization of a branched $(1 \rightarrow 6)$-alpha-mannan and beta-glucans isolated from the fruiting bodies of Cantharellus cibarius. Carbohydrate Polymers, 146 197-207.

Pettolino, F. A., Walsh, C., Fincher, G. B., \& Bacic, A. (2012). Determining the polysaccharide composition of plant cell walls. Nature Protocols, 7(9), 1590-1607.

Prados-Rosales, R., Toriola, S., Nakouzi, A., Chatterjee, S., Stark, R., Gerfen, G., ... Casadevall, A. (2015). Structural characterization of melanin pigments from commercial preparations of the edible mushroom Auricularia auricula. Journal of Agricultural and Food Chemistry, 63(33), 7326-7332.

Qiao, Y., Giannopoulou, E. G., Chan, C. H., Park, S. H., Gong, S., Chen, J., ... Ivashkiv, L. B. (2013). Synergistic activation of inflammatory cytokine genes by interferongamma-induced chromatin remodeling and toll-like receptor signaling. Immunity, 39(3), 454-469.

Rhee, S. J., Cho, S. Y., Kim, K. M., Cha, D.-S., \& Park, H.-J. (2008). A comparative study of analytical methods for alkali-soluble $\beta$-glucan in medicinal mushroom, Chaga (Inonotus obliquus). LWT- Food Science and Technology, 41(3), 545-549.

Saar, M. (1991). Fungi in Khanty folk medicine. Journal of Ethnopharmacology, 175-179.

Schwartz, B., \& Hadar, Y. (2014). Possible mechanisms of action of mushroom-derived glucans on inflammatory bowel disease and associated cancer. Annals of Translational Medicine, 2(2), 19.

Shashkina, M. Y., Shashkin, P. N., \& Sergeev, A. V. (2006). Chemical and medicobiological properties of chaga (review). Pharmaceutical Chemistry Journal, 40(10), 560-568.

Shikov, A. N., Pozharitskaya, O. N., Makarov, V. G., Wagner, H., Verpoorte, R., \& Heinrich, M. (2014). Medicinal plants of the Russian pharmacopoeia; their history and applications. Journal of Ethnopharmacology, 154(3), 481-536.

Smiderle, F. R., Olsen, L. M., Carbonero, E. R., Marcon, R., Baggio, C. H., Freitas, C. S., ... Iacomini, M. (2008). A 3-O-methylated mannogalactan from Pleurotus pulmonarius: Structure and antinociceptive effect. Phytochemistry, 69(15), 2731-2736.

Staudacher, E. (2012). Methylation - an uncommon modification of glycans. The Journal of Biological Chemistry, 393(8), 675-685.

Swain, T., \& Hillis, W. E. (1959). The phenolic constituents of Prunus domestica. I. - the quantitative analysis of phenolic constituents. Journal of the Science of Food and Agriculture, 10(1), 63-68.

Totemeyer, S., Sheppard, M., Lloyd, A., Roper, D., Dowson, C., Underhill, D., ... Bryant, C. (2006). IFN- $\gamma$ enhances production of nitric oxide from macrophages via a mechanism that depends on nucleotide oligomerization domain-2. The Journal of Immunology, 176(8), 4804-4810.

Wei, S., \& Van Griensven, L. J. (2008). Pro- and antioxidative properties of medicinal mushroom extracts. International Journal of Medicinal Mushrooms, 10(4), 315-324.

Whistler, R. L., \& Wolfrom, M. L. (1963). Reactions of carbohydrates. New York: Academi Press Inc.

Wiercigroch, E., Szafraniec, E., Czamara, K., Pacia, M. Z., Majzner, K., Kochan, K., ... Malek, K. (2017). Raman and infrared spectroscopy of carbohydrates: A review. Spectrochimica Acta Part A: Molecular and Biomolecular Spectroscopy, 185, 317-335.

Won, D. P., Lee, J. S., Kwon, D. S., Lee, K. E., Shin, W. C., \& Hong, E. K. (2011) Immunostimulating activity by polysaccharides isolated from fruiting body of Inonotus obliquus. Molecules and Cells, 31(2), 165-173.

Xu, X., Quan, L., \& Shen, M. (2015). Effect of chemicals on production, composition an antioxidant activity of polysaccharides of Inonotus obliquus. International Journal of Biological Macromolecules, 77, 143-150.

Yang, Y., Zhang, J., Liu, Y., Tang, Q., Zhao, Z., \& Xia, W. (2007). Structural elucidation of a 3-O-methyl-D-galactose-containing neutral polysaccharide from the fruiting bodie of Phellinus igniarius. Carbohydrate Research, 342(8), 1063-1070.

Yapo, B. M. (2011). Pectic substances: From simple pectic polysaccharides to complex pectins-a new hypothetical model. Carbohydrate Polymers, 86(2), 373-385.

Youn, M. J., Kim, J. K., Park, S. Y., Kim, Y., Park, C., Kim, E. S., .. Park, R. (2009). Potential anticancer properties of the water extract of Inonotus obliquus by induction of apoptosis in melanoma B16-F10 cells. Journal of Ethnopharmacology, 121(2), 
$221-228$.

Zevenhuizen, L. P. T. M. (1997). Succinoglycan and galactoglucan. Carbohydrate Polymers, 33, 139-144.

Zhang, M., Cui, S. W., Cheung, P. C. K., \& Wang, Q. (2007). Antitumor polysaccharides from mushrooms: A review on their isolation process, structural characteristics and antitumor activity. Trends in Food Science \& Technology, 18(1), 4-19.
Zhao, F., Mai, Q., Ma, J., Xu, M., Wang, X., Cui, T., ... Han, G. (2015). Triterpenoids from Inonotus obliquus and their antitumor activities. Fitoterapia, 101, 34-40.

Zheng, W., Miao, K., Liu, Y., Zhao, Y., Zhang, M., Pan, S., ... Dai, Y. (2010). Chemical diversity of biologically active metabolites in the sclerotia of Inonotus obliquus and submerged culture strategies for up-regulating their production. Applied Microbiology and Biotechnology, 87(4), 1237-1254. 



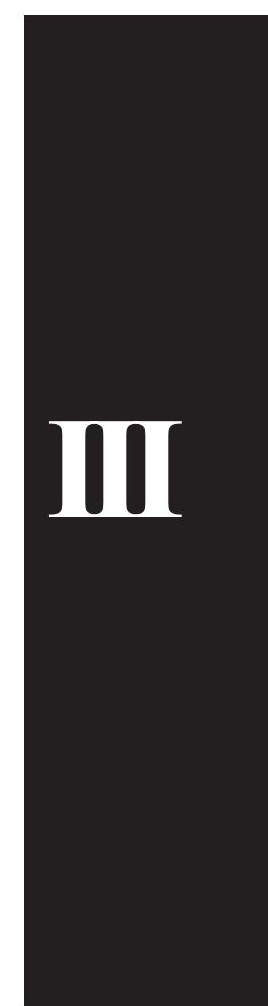





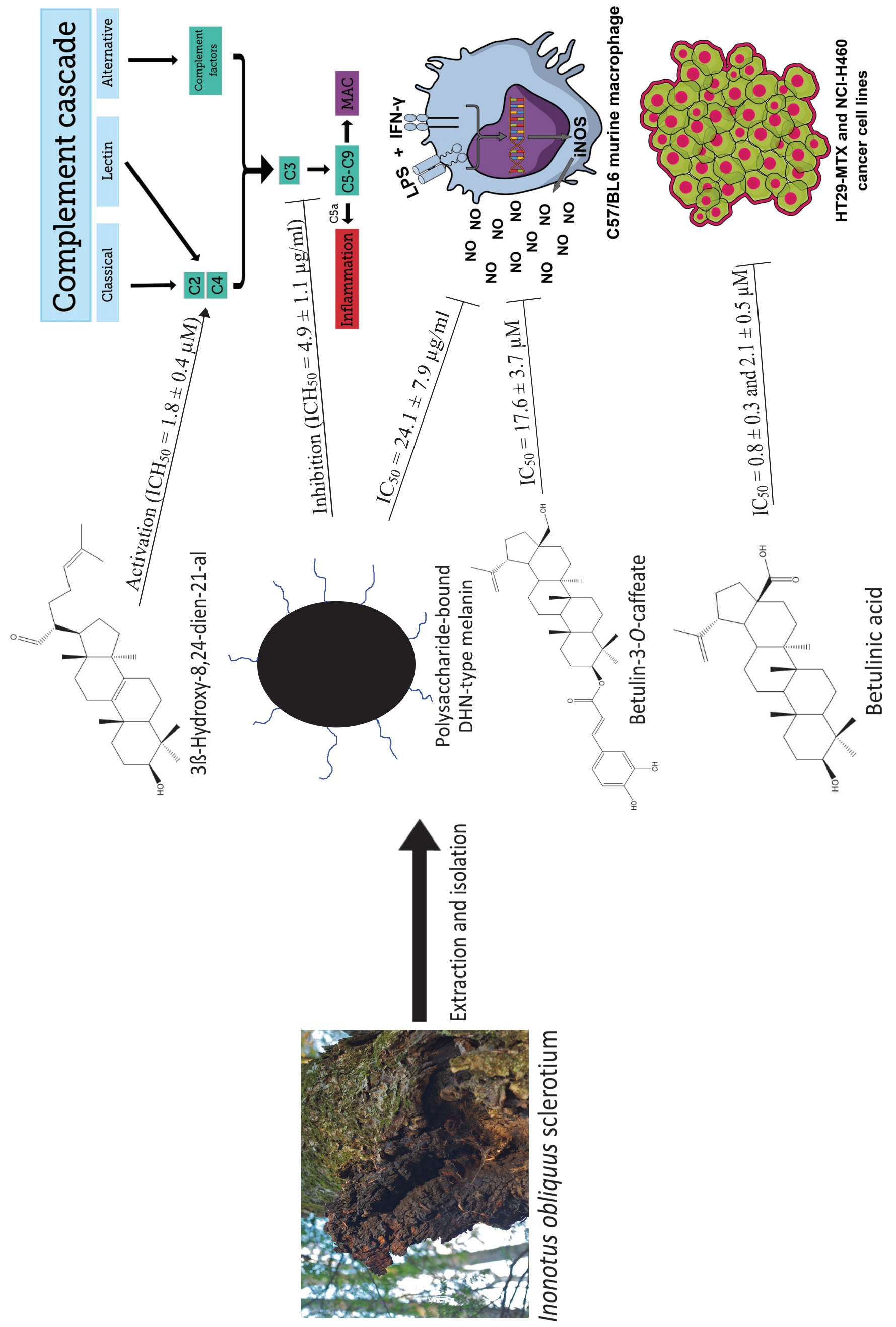





\title{
Bioactive triterpenoids and water-soluble melanin from Inonotus obliquus (Chaga) with immunomodulatory activity
}

\author{
Christian W. Wold ${ }^{\mathrm{a}, *}$, William H. Gerwick ${ }^{\mathrm{b}}$, Helle Wangensteen ${ }^{\mathrm{a}, 1}$, Kari T. Inngjerdingen ${ }^{\mathrm{a}, 1}$ \\ ${ }^{a}$ Section for Pharmaceutical Chemistry, Department of Pharmacy, University of Oslo, Norway \\ ${ }^{\mathrm{b}}$ Scripps Institution of Oceanography and Skaggs School of Pharmacy and Pharmaceutical Sciences, University of California San Diego, La Jolla, CA 92093, USA
}

\section{A R T I C L E I N F O}

\section{Keywords:}

Inonotus obliquus (Chaga)

Melanin

Triterpenoids

Anti-inflammatory

Complement

Cytotoxicity

\begin{abstract}
A B S T R A C T
The fungus Inonotus obliquus has historically been used in traditional medicine in Europe and Asia. A melanin fraction and six triterpenoids were obtained from I. obliquus sclerotia, and evaluated in various bioassays including for immunomodulatory, cytotoxicity and enzyme-interacting properties. The water-soluble, nitrogenfree melanin fraction and the triterpenoids 3ß-hydroxy-8,24-dien-21-al (1) and inotodiol (2) displayed potent activity in a human complement assay. The melanin fraction inhibited the complement cascade, whereas $\mathbf{1}$ and $\mathbf{2}$ activated the same cascade. Compound 2, as well as betulinic acid (3) and betulin (4) had anti-proliferative properties against the colon adenocarcinoma cell line HT29-MTX. Further, the melanin fraction and betulin-3-Ocaffeate (6) reduced nitric oxide production in primary murine macrophages. Furthermore, the metabolites were nontoxic against the common gut bacteria $E$. coli and $B$. subtilis. The results demonstrate the anti-inflammatory and immunomodulatory effects of $I$. obliquus melanin and triterpenoids, which could potentially justify the consumption of this increasingly popular "edible" fungus.
\end{abstract}

\section{Introduction}

Inonotus obliquus (Fr.) Pilát, a rather unusual polypore fungus growing on birch trees in northern latitudes, has been used in traditional medicine in Northern Europe, Canada and Russia since the 16th century against a wide range of illnesses such as tuberculosis, heart disease, cancer and gastrointestinal disorders (Shikov et al., 2014). The last decade or so, I. obliquus has become increasingly popular in the US and EU, where the fungus is now readily available as a food supplement in the form of water- or ethanol-based extracts. Anti-inflammatory, antioxidant, and anti-cancer effects of extracts and isolated substances from I. obliquus have been reported, likely caused by polyphenols, triterpenoids or polysaccharides (Du, Zhu, \& Xu, 2018; Wang et al., 2018; Zheng et al., 2010). However, little information exists on the chemical and biological properties of water-soluble melanin from I. obliquus. As this fungus is most commonly consumed as water extracts containing large amounts of melanin (Shikov et al., 2014; Wold et al., 2018), the chemical structure of the isolated melanin pigment was characterized in more detail, and its biological properties, including antimicrobial and immunological effects, were investigated. In addition to the lack of information on I. obliquus melanin, most of the research on triterpenoids from $I$. obliquus has focused on their anti-proliferative and antiviral effects. As such, there is a knowledge gap when it comes to other types of bioactivities from these constituents, which are usually present in ethanol-based I. obliquus products on the market (Shikov et al., 2014; Zhao et al., 2015). The importance of discovering new types of bioactivities for known molecules can be exemplified by betulin, a triterpenoid thought to have limited clinical potential due to its poor bioavailability in humans (Furtado et al., 2017). Recently, a birch bark preparation called Episalvan, containing 80\% betulin, was approved as a prescription drug by the European Medicines Agency (EMA) for the treatment of superficial skin wounds (epidermis and upper dermis) and second-degree burn wounds, where it appears to have superior effects compared to other treatments (Scheffler, 2019). In the present study, we characterized and screened extracts, isolated triterpenoids and a water-soluble melanin fraction from I. obliquus in a wide range of immunological, enzymatic and anti-proliferative assays, in order to discover new types of biological activities from these increasingly

Abbreviations: $\mathrm{IO}_{\mathrm{I}}$, Inonotus obliquus interior part; $\mathrm{IO}_{\mathrm{E}}$, Inonotus obliquus exterior part; NMR, nuclear magnetic resonance; DHN, 1,8-dihydroxynaphthalene; LPS, lipopolysaccharide; IFN $\gamma$, interferon gamma; NO, nitric oxide; DCM, dichloromethane; MeOH, methanol; EtOH, ethanol; DMSO, dimethyl sulfoxide; 15-LOX, 15lipoxygenase; XO, xanthine oxidase; DPPH, 2,2-diphenyl-1-picrylhydrazyl

* Corresponding author.

E-mail address: c.w.wold@farmasi.uio.no (C.W. Wold).

${ }^{1}$ These authors contributed equally to this work. 
consumed fungal metabolites.

\section{Materials and methods}

\subsection{General experimental procedures}

Preparative and analytical reverse phase high performance liquid chromatography (HPLC) was performed on Varian ProStar and Hitachi LaChrom Elite instruments, respectively, using Kinetex C18 columns [(preparative: $150 \times 21.3 \mathrm{~mm}$; analytical: $150 \times 4.6 \mathrm{~mm}$ ), $100 \AA$, $5 \mu \mathrm{m}$, Phenomenex]. Preparative thin-layer chromatography (TLC) was performed using standard silica gel plates $\left(\mathrm{F}_{254}, 20 \times 20 \mathrm{~cm}, 0.25 \mathrm{~mm}\right.$, Merck), or a Chromatotron ${ }^{\circledR}$ system (model 792413, Harrison Research) in a $\mathrm{N}_{2}$ atmosphere, using silica gel $60 \mathrm{PF}_{254}$ containing gypsum ( $2 \mathrm{~mm}$, Merck) as stationary phase. All fractionations were monitored by TLC and ${ }^{1} \mathrm{H}$ nuclear magnetic resonance (NMR) spectroscopy. ${ }^{1} \mathrm{H},{ }^{13} \mathrm{C}$ and 2D NMR spectra were recorded on a Bruker Avance II 600 spectrometer (600 MHz for ${ }^{1} \mathrm{H}$ and $150 \mathrm{MHz}$ for ${ }^{13} \mathrm{C}$, Bruker $\mathrm{GmbH}$ ) equipped with a $5 \mathrm{~mm} \mathrm{TCI}{ }^{1} \mathrm{H} /\left({ }^{13} \mathrm{C},{ }^{15} \mathrm{~N}\right.$ ) cryoprobe, using $\mathrm{CDCl}_{3}$ or $\mathrm{CD}_{3} \mathrm{OD}$ (both from Sigma-Aldrich) as solvents for the triterpenoids and $\mathrm{NaOH}(0.2 \mathrm{M})$ in $\mathrm{D}_{2} \mathrm{O}$ (Sigma-Aldrich) for the acid-treated, water-insoluble melanin. Tetramethylsilane (TMS, Sigma-Aldrich) or trimethylsilylpropanoic acid (TSP, Sigma-Aldrich) were used as internal standards. Infrared (IR) spectra of the melanin fraction were obtained on a Nicolet ${ }^{\mathrm{TM}}$ FT-IR Spectrometer (Thermo Fischer Scientific). High-resolution electrospray ionization mass spectrometry (HRESIMS) data were obtained using an Agilent 6230 Accurate-Mass TOFMS (Agilent) or Waters QTOF $2 \mathrm{~W}$ (Waters). Molecular weight of the melanin fraction was determined by gel filtration HPLC (UV-vis $223 \mathrm{~nm}$ ), using a Diol-300 column $\left(500 \times 6 \mathrm{~mm}, 5 \mu \mathrm{m}\right.$, YMC), eluting with $50 \mathrm{mM} \mathrm{Na}_{2} \mathrm{SO}_{4}$ and using dextran polymers as standards $(11.7-475 \mathrm{kDa}$, detection by refractive index (RI) detector). To investigate for presence of carbohydrates in the melanin fractions, the method by Chambers and Clamp with some modifications was utilized (Wold et al., 2018). In short, melanin fractions (both water-soluble and water-insoluble) were subjected to acid methanolysis with $\mathrm{HCl}(3 \mathrm{M})$ in methanol $\left(\mathrm{MeOH}, 24 \mathrm{~h}, 80{ }^{\circ} \mathrm{C}\right)$. Trimethylsilylated (TMS) derivatives of the methyl glycosides were analyzed by capillary gas chromatography on a Trace ${ }^{\mathrm{TM}} 1300$ GC (Thermo Scientific). Mannitol was used as an internal standard to determine the amount of each monosaccharide. For combustion analysis of melanin, samples were analyzed using a PerkinElmer 2400 Series II CHNS/O Element Analyzer, using Sepia officinalis eumelanin as a control. An acid-treated, carbohydrate-devoid melanin fraction was utilized for both the combustion analysis and the analysis of melanin degradation products, and for some of the NMR spectroscopy experiments. To analyze degradation products of melanin, a method developed by Ito et al was used (S. Ito et al., 2011). The melanin fraction (10 mg) was suspended in $1 \mathrm{~mL}$ distilled water before $\mathrm{K}_{2} \mathrm{CO}_{3}(3.75 \mathrm{~mL}, 1 \mathrm{M})$ and $\mathrm{H}_{2} \mathrm{O}_{2}(250 \mu \mathrm{L}, 30 \%)$ were added. The sample was mixed vigorously for $20 \mathrm{~h}$ at $25^{\circ} \mathrm{C}$, before $\mathrm{Na}_{2} \mathrm{SO}_{4}(500 \mu \mathrm{L}, 10 \%)$ and $\mathrm{HCl}(1.4 \mathrm{~mL}, 6 \mathrm{M})$ were added. The sample was centrifuged (4000 g, $1 \mathrm{~min}$ ), filtered (Millipore, $0.45 \mu \mathrm{m}$ ) and analyzed by HPLC (Kinetex C18, analytical) using potassium phosphate buffer/MeOH (99:1) as a mobile phase $(0.1 \mathrm{M}, \mathrm{pH}$ 2.1 , flow $0.8 \mathrm{~mL} / \mathrm{min}$ ). The degradation mixture was further analyzed by NMR spectroscopy after removal of salts and buffers by stepwise $\mathrm{MeOH}$ extraction of the dried degradation mixture, using small amounts of $\mathrm{MeOH}$ each time and finally dissolving it in $\mathrm{CD}_{3} \mathrm{OD}$.

\subsection{Fungal material}

Sclerotia of I. obliquus were harvested from a birch tree in Oslo, Norway (Coordinates (WGS84): N 59 57'01.4, E 10 41'32.0), in October 2015, and verified by Prof. Klaus Høiland (Dept. of Biosciences, UiO, Norway). A voucher specimen (specimen no. CW20151020) was deposited in the Pharmacognosy section, Department of Pharmacy, University of Oslo, Norway. Prior to extraction, the fungal material was separated into two parts distinguished by the difference in texture and color, a brown, soft interior part $\left(\mathrm{IO}_{\mathrm{I}}\right)$ and a black, hard exterior part $\left(\mathrm{IO}_{\mathrm{E}}\right)$. These separate parts were cut into small pieces and lyophilized. The separate parts were then ground into a finely dispersed powder using an industrial blender (RAW $\left.{ }^{\circledR} \mathrm{X} 1500\right)$.

\subsection{Extraction and isolation of triterpenoids}

The two fungal sections (227 g/131 g interior/exterior parts, respectively) were extracted with dichloromethane (DCM) under reflux in a Soxhlet extractor for $48 \mathrm{~h}$ to provide two DCM extracts $(4.1 \mathrm{~g} / 1.5 \mathrm{~g}$ interior/exterior). The DCM extracts were then fractionated by vacuum liquid chromatography (VLC) as previously described (Shao et al., 2015). Briefly, the extracts were applied to a silica gel column (Silica type H, Sigma, prepared in $n$-hexane) and fractionated under reduced pressure by solvents of increasing polarity. The fractionation was carried out using $n$-hexane, ethyl acetate (EtOAc) and $\mathrm{MeOH} \mathrm{(1:0:0} \rightarrow$ 9:1:0 $\rightarrow$ 4:1:0 $\rightarrow$ 3:2:0 $\rightarrow$ 2:3:0 $\rightarrow$ 1:4:0 $\rightarrow$ 0:1:0 $\rightarrow$ 0:3:1 $\rightarrow$ 0:0:1, $150 \mathrm{~mL}$ total volume for each fraction), to give fractions $\mathrm{IO}_{\mathrm{I}} / \mathrm{IO}_{\mathrm{E}}-\mathrm{F} 1-\mathrm{F} 9$ for each fungal part. $\mathrm{IO}_{\mathrm{I}}-\mathrm{F} 4$ (337 $\mathrm{mg}$ ) was further fractionated using a silica gel flash column (VersaPak spherical silica cartridge, $150 \mathrm{~mL}$ ), using a stepwise gradient of DCM-EtOAc $(1: 0 \rightarrow 9: 1 \rightarrow 4: 1 \rightarrow 13: 7 \rightarrow$ $1: 1 \rightarrow 0: 1,50 \mathrm{~mL}$ total volume for each fraction) to give fractions $\mathrm{IO}_{\mathrm{I}^{-}}$ F4-V1 - V6. IO $\mathrm{I}^{-\mathrm{F} 4-\mathrm{V} 2}(69.7 \mathrm{mg})$ was further fractionated using the Chromatotron ${ }^{\circledR}$ silica gel system (Bergheim, Malterud, \& Anthonsen, 1991). Briefly, the sample was dissolved in DCM and eluted with DCMEtOAc $(1: 0 \rightarrow 19: 1 \rightarrow 9: 1 \rightarrow 4: 1 \rightarrow 1: 1,25 \mathrm{~mL}$ total volume for each fraction) to give fractions $\mathrm{IO}_{\mathrm{I}^{-}} \mathrm{F} 4-\mathrm{V} 2-\mathrm{C} 1$ - C5. $\mathrm{IO}_{\mathrm{I}}-\mathrm{F} 4-\mathrm{V} 2-\mathrm{C} 2(41.9 \mathrm{mg})$ was further purified on the Chromatotron ${ }^{\circledast}$ with DCM, yielding 1 (23.5 mg). $\mathrm{IO}_{\mathrm{I}^{-}}-\mathrm{F} 4-\mathrm{V} 2-\mathrm{C} 4$ was identified as compound $2(4.7 \mathrm{mg}) . \mathrm{IO}_{\mathrm{I}^{-}}$ F4-V2-C5 (8.9 mg) was further purified by TLC (DCM-hexane 1:1) to give compound $3(4.8 \mathrm{mg})$. Due to some impurities in this fraction (the purity was found to be $85-90 \%$ ), pure compound 3 (betulinic acid) was purchased (Sigma-Aldrich) for parallel use in the bioassays. Next, $\mathrm{IO}_{\mathbf{I}^{-}}$ F5 (359 mg) was subjected to preparative HPLC (acetonitrile- $\mathrm{H}_{2} \mathrm{O} 1: 1$ to 99:1, linear gradient, $30 \mathrm{~min}$ ) to give fractions $\mathrm{IO}_{\mathrm{I}}-\mathrm{F} 5-\mathrm{H} 1-\mathrm{H6}$. $\mathrm{IO}_{\mathrm{I}}-\mathrm{F} 5-$ $\mathrm{H} 2\left(\mathrm{t}_{\mathrm{R}} 20.9 \mathrm{~min}, 37.2 \mathrm{mg}\right)$ was further fractionated with HPLC (MeOH$\mathrm{H}_{2} \mathrm{O} 4: 1$ to $99: 1$, linear gradient, $30 \mathrm{~min}$ ), giving fractions $\mathrm{IO}_{\mathrm{I}}-\mathrm{F} 5-\mathrm{H} 2-\mathrm{A} 1$ - A4. $\mathrm{IO}_{\mathrm{I}}-\mathrm{F} 5-\mathrm{H} 2-\mathrm{A} 1\left(\mathrm{t}_{\mathrm{R}} 14.7 \mathrm{~min}\right)$ was identified as compound 4 (7 mg). $\mathrm{IO}_{\mathrm{I}^{-}} \mathrm{F} 5-\mathrm{H} 2-\mathrm{A} 4\left(\mathrm{t}_{\mathrm{R}} 16.7 \mathrm{~min}\right)$ was identified as compound $5(3.8 \mathrm{mg})$. Ultimately, $\mathrm{IO}_{\mathrm{E}}-\mathrm{F} 5$ from the exterior part of the fungus (192 $\mathrm{mg}$ ) was subjected to reverse phase HPLC (acetonitrile- $\mathrm{H}_{2} \mathrm{O}$ 1:1 to 99:1, $40 \mathrm{~min}$ ), giving fractions $\mathrm{IO}_{\mathrm{E}}-\mathrm{F} 5-\mathrm{H} 1$ - $\mathrm{H} 5$. $\mathrm{IO}_{\mathrm{E}}-\mathrm{F} 5-\mathrm{H} 4\left(\mathrm{t}_{\mathrm{R}} 22.8 \mathrm{~min}, 6.5 \mathrm{mg}\right.$ ) was further purified by TLC (silica gel, hexane-acetone $3: 1$ ) to yield compound 6 (3 mg).

\subsection{Isolation of water-soluble melanin}

The fungal residues from the two sections after extraction with DCM were extracted with $\mathrm{MeOH}$ under reflux for $48 \mathrm{~h}$ in a Soxhlet extractor to obtain two $\mathrm{MeOH}$ extracts $\left(\mathrm{IO}_{\mathrm{I}}-\mathrm{MeOH}\right.$ and $\left.\mathrm{IO}_{\mathrm{E}}-\mathrm{MeOH}\right)$. The remaining residue of the interior part was extracted with distilled water $\left(\mathrm{dH}_{2} \mathrm{O}, 100{ }^{\circ} \mathrm{C}, 3 \mathrm{x}, 2 \mathrm{~h}\right)$, treated with pancreatin to remove proteins ( $3 \mathrm{~h}, 37^{\circ} \mathrm{C}$, Sigma), precipitated with ethanol $\left(70 \%, 48 \mathrm{~h}, 4{ }^{\circ} \mathrm{C}\right.$ ) and dialyzed (cut-off $3500 \mathrm{Da}, 72 \mathrm{~h}, 4{ }^{\circ} \mathrm{C}$ ) to yield an extract $\left(\mathrm{IO}_{\mathrm{I}^{-}}-\mathrm{W}, 25.3 \mathrm{~g}\right.$ ) rich in polysaccharides and melanin (Wold et al., 2018). $\mathrm{IO}_{\mathrm{I}}-\mathrm{W}$ (1 g) was then treated with diluted $\mathrm{HCl}\left(10 \mathrm{mM}, 30 \mathrm{~min}, 37^{\circ} \mathrm{C}, \mathrm{pH} 2\right)$, allowing the pigment to precipitate. The supernatant (containing carbohydrates) was discarded and the residue was washed several times with diluted $\mathrm{HCl}$ before it was neutralized to $\mathrm{pH} 7$ with $\mathrm{NaOH}$ (1 M), dialyzed and lyophilized to yield a water-soluble melanin fraction $(600 \mathrm{mg})$. To analyze the structure of melanin further, the water-soluble melanin fraction (300 mg) was treated with $\mathrm{HCl}\left(6 \mathrm{M}, 24 \mathrm{~h}, 100{ }^{\circ} \mathrm{C}\right)$, washed several times with distilled water and lyophilized, to yield an acid-treated, water-insoluble melanin fraction $(282 \mathrm{mg})$. 


\subsection{Bioassays}

Bioassays were carried out in triplicates, in three separate experiments, and recorded and analyzed on a SpectraMax 190 Microplate Reader (Molecular Devices) unless otherwise stated. For all bioassays, samples were dissolved in dimethyl sulfoxide (DMSO) and tested in four to seven different concentrations to establish dose-dependent effects and $\mathrm{IC}_{50}$ values. For the assays involving cells or complement proteins, the final concentration of DMSO was $0.5 \% \mathrm{v} / \mathrm{v}$ (for the highest concentration of sample) or lower. For the enzymatic assays, the final concentration of DMSO was $1.67 \% \mathrm{v} / \mathrm{v}$.

\subsubsection{Cancer cell lines and bone marrow-derived murine macrophages}

The NCI-H460 cell line (ATCC ${ }^{\circledR}$, H460) was cultured using RPMI1640 medium containing fetal bovine serum (FBS, 10\%, Sigma) and penicillin-streptomycin (Pen-Strep, 1\%, Sigma). The HT29-MTX cell line was cultured using DMEM- GlutaMax ${ }^{\mathrm{TM}}$ medium containing FBS (10\%, Sigma) and Pen-Strep (1\%). This cell line was a gift from Dr. Thécla Lesuffleur (Inserm Umr S938, Paris, France) and is resistant to methotrexate at $1 \mu \mathrm{M}$ (Lesuffleur et al., 1993). The cells were passaged at $60-80 \%$ confluency to prevent mucus production. Murine bone marrow-derived macrophages from C57BL/6 mice (BL6 macrophages) were isolated by flushing the bone-marrow of mice and differentiating macrophages using L929-conditioned medium (CM, 30\%) as previously described (Muller et al., 2017). One vial of cells $\left(5 \times 10^{6}\right.$ cells $\left./ \mathrm{mL}\right)$ was thawed before each experiment and maintained with RPMI-1640 medium containing FBS (10\%, BioChrom) and L929-conditioned media (CM, 10\%).

\subsubsection{Anti-inflammatory activity in LPS + IFN $\gamma$-activated macrophages}

The anti-inflammatory activity was investigated by measuring the inhibition of nitric oxide (NO) production in C57BL/6 primary macrophages, induced by lipopolysaccharide (LPS) and interferon gamma (IFN $\gamma$ ). The assay was carried out using the Griess assay as previously described, with some modifications (Ho, Wangensteen, \& Barsett, 2017). Briefly, macrophages were seeded in 96-wells plates at a density of $5 \times 10^{4}$ /well, and pre-incubated for one hour $\left(37^{\circ} \mathrm{C}, 5 \% \mathrm{CO}_{2}\right)$ with test compounds before LPS (500 ng/mL, Escherichia coli O55:B5, Sigma) and IFN $\gamma$ ( $20 \mathrm{ng} / \mathrm{mL}$, Peprotech) were added. The cells were incubated for $24 \mathrm{~h}$, and the amount of nitrite in the cell supernatants was measured at $\mathrm{A}_{540}$ after addition of Griess reagents $\mathrm{A}$ and $\mathrm{B}$, using a standard curve of $\mathrm{NaNO}_{2}$. Quercetin was used as a positive control. NO inhibition was expressed as the percentage decrease in NO production compared to the samples treated with LPS + IFN $\gamma$ (containing 0.5\% v/v DMSO).

\subsubsection{Cell viability assay}

Cell viability was determined by the MTT Assay (Cell Proliferation Kit I, Roche), measuring formazan salt formation in the cell media after $24 \mathrm{~h}$, according to the manufacturers protocol (Roche, , 2016). The results were calculated by subtracting $\mathrm{A}_{690}$ values from $\mathrm{A}_{570}$ values for each sample and comparing to the untreated control (containing $0.5 \%$ v/v DMSO). For the experiments with NCI-H460 and HT29-MTX cell lines, podophyllotoxin (Sigma) was used as positive control. For the measurement of viability subsequent to the anti-inflammation assay, DMSO $(20 \% \mathrm{v} / \mathrm{v})$ was used as a control for complete reduction in viability.

\subsubsection{Complement fixation assay}

The complement fixation assay is based on inhibition of hemolysis of antibody-sensitized sheep red blood cells (SRBC) by human sera, as described previously (Inngjerdingen, Coulibaly, Diallo, Michaelsen, \& Paulsen, 2006). Briefly, samples were dissolved in DMSO to stock concentrations $(5 \mathrm{mM})$ and diluted in veronal buffered saline $(\mathrm{pH} 7.2$, Virion/Serion) containing bovine serum albumin (Sigma). Serum titration was carried out before the experiments to give a serum concentration that gave $50 \%$ hemolysis of the blood cells. To determine if the samples either inhibited or activated human complement, the serum was incubated $\left(37^{\circ} \mathrm{C}\right)$ with samples for either $0 \mathrm{~min}$ (inhibition) or $30 \mathrm{~min}$ (activation) before addition of blood cells $\left(30 \mathrm{~min}, 37^{\circ} \mathrm{C}\right.$ ), according to a method developed by Alban et al (Alban, Classen, Brunner, \& Blaschek, 2002). The $\mathrm{A}_{405}$ values were used to calculate percent inhibition of hemolysis by comparing to the untreated controls that gave $\sim 50 \%$ hemolysis. A pectic polysaccharide isolated from Biophytum petersianum (BPII) (Inngjerdingen et al., 2006) and heparin (Heparin Sodium Salt, Sigma) were used as positive controls.

\subsubsection{Determination of complement pathway}

To investigate which of the three complement pathways the samples interacted with (classical, lectin or alternative), the WIESLAB ${ }^{\circledR}$ Complement system Screen kit (Svar Life Science) was utilized (SvarLifeScience, , 2015). The experiment was carried out according to protocol, with an additional incubation step $\left(30 \mathrm{~min}, 37^{\circ} \mathrm{C}\right)$ of the isolated samples with serum before adding them to the wells containing activators of each pathway (IgM, mannan or LPS for the classical, lectin and alternative pathways, respectively). $\mathrm{A}_{405}$ values were used to calculate percent interaction with the three complement pathways by comparing to the serum controls. To ensure a complete interaction with complement components, a compound concentration of $50 \mu \mathrm{g} / \mathrm{mL}$ was used. The experiment was carried out once, in technical duplicates using two different sera from healthy human subjects. The positive control was a serum provided by Svar Life Science, known to activate all three pathways. The results are given as relative interaction with each pathway compared to the positive control.

\subsubsection{Inhibition of xanthine oxidase}

The ability of extracts and isolated compounds to inhibit the activity of xanthine oxidase (XO, from bovine milk, Sigma) was investigated as previously described (Braunlich et al., 2013). The assay was adopted to a 96-well format, using UV-transparent well plates (Corning). Briefly, the samples were mixed with $\mathrm{XO}(6.8 \mathrm{mU} / \mathrm{mL})$ in phosphate buffer (50 mM, pH 7.4) and incubated $\left(5 \mathrm{~min}, 25^{\circ} \mathrm{C}\right)$, before xanthine $(50 \mu \mathrm{M})$ was added as a substrate. The increase in $\mathrm{A}_{290}$ for 5 min was measured and used to calculate enzyme inhibition by comparing with the untreated enzyme control. Allopurinol was used as a positive control.

\subsubsection{Inhibition of 15-lipoxygenase}

The ability of extracts and isolated compounds to inhibit the activity of 15-lipoxygenase from soybean (15-LOX, Sigma) was investigated as previously described (Braunlich et al., 2013). The assay was adopted to a 96-well format, using UV-transparent well plates (Corning). Briefly, the samples were mixed with $15-\mathrm{LOX}(200 \mathrm{U} / \mathrm{mL}$ final concentration) in borate buffer $\left(0.2 \mathrm{M}, \mathrm{pH}\right.$ 9) and incubated $\left(5 \mathrm{~min}, 25^{\circ} \mathrm{C}\right)$, before linoleic acid $(134 \mu \mathrm{M})$ was added as a substrate. The increase in $\mathrm{A}_{234}$ for $1.5 \mathrm{~min}$ was measured and used to calculate enzyme inhibition by comparing with the untreated enzyme control. Quercetin was used as a positive control.

\subsubsection{Inhibition of acetylcholinesterase}

The ability of extracts and isolated compounds to inhibit the activity of acetylcholinesterase from E. electricus (Sigma) was investigated as previously described, with minor modifications (Passos et al., 2013). Briefly, Ellman's reagent (0.18 mM, Sigma) in phosphate buffer (50 mM, pH 7.4) was mixed with samples and acetylcholinesterase (66 mU/mL final concentration) before acetylthiocholine iodine (ATCI, $300 \mu \mathrm{M}$ ) was added as a substrate. $\mathrm{A}_{415}$ after 6 min was measured and used to calculate enzyme inhibition by comparing with the untreated enzyme control. Tacrine and galanthamine were used as positive controls.

\subsubsection{Antimicrobial assay}

Antimicrobial activity of extracts and isolated compounds was assessed using the disk diffusion method as previously described 
(Wangensteen et al., 2006). The following bacterial and fungal strains were used: Escherichia coli ATCC 25922, Staphylococcus aureus ATCC 25923, Bacillus subtilis ATCC 6633, Pseudomonas aeruginosa ATCC 15442, Candida albicans ATCC 10,231 (all from Thermo Scientific). Mc Fairland standard no. 2 was used to standardize the amount of bacteria and yeast, and $50 \mu \mathrm{L}$ sample at various concentrations were loaded onto the discs, using DCM as a solvent. Chloramphenicol, gentamicin and sulconazole (all from Sigma) were used as positive controls. The assay was carried out in duplicate.

\section{6. $D P P H$ radical scavenging}

The 2,2-diphenyl-1-dipicrylhydrazyl (DPPH) radical scavenging activity of extracts and isolated compounds was investigated as previously described (Herald, Gadgil, \& Tilley, 2012). Briefly, DPPH solution $(36 \mu \mathrm{g} / \mathrm{mL})$ and samples were mixed and $A_{517}(5 \mathrm{~min})$ was measured. Results were calculated by comparing samples with an untreated DPPH control. Quercetin was used as a positive control. The experiment was carried out once with technical triplicates.

\subsection{Statistics}

Statistical analysis was conducted by using the GraphPad Prism 8 software (GraphPad). Values from control samples were used as a reference to each individual value. The data were analyzed using one-way ANOVA test, followed by Dunnett's test for comparison of multiple samples. Values were expressed as mean \pm SD of at least three separate experiments, if not otherwise stated.

\section{Results and discussion}

\subsection{Triterpenoid identification}

Six triterpenoids (Fig. 1) were obtained from I. obliquus after purification of the DCM extracts from the interior $\left(\mathrm{IO}_{\mathrm{I}}-\mathrm{DCM}\right)$ and the exterior $\left(\mathrm{IO}_{\mathrm{E}}-\mathrm{DCM}\right)$ part of the fungus. Along with 3 $\beta$-hydroxy-8,24-dien21-al (1) (Kahlos, Hiltunen, \& Schantz, 1984), inotodiol (2) (Shin, Tamai, \& Terazawa, 2000), betulinic acid (3) (Sholichin, Yamasaki, Kasai, \& Tanaka, 1980), betulin (4) (Fuchino, Satoh, \& Tanaka, 1995) and trametenolic acid (5) (Shin et al., 2000), previously isolated from $I$. obliquus, we report here betulin-3-O-caffeate (6) (Fuchino et al., 1995) for the first time in this species. Compound $\mathbf{1}$ is to our knowledge only reported to be found in I. obliquus (SciFinder, chemical structure search Feb 3-2020). Spectroscopic data of 1-6 were compared with values reported in the literature and confirmed by HRESIMS. For NMR- and mass spectra of compound 6, see Figs. S1, S2 and S5 in supplementary information. For ${ }^{1} \mathrm{H}$ and ${ }^{13} \mathrm{C}$ NMR spectra of compounds $\mathbf{1 - 5}$, see Figs. S26-S36.

\subsection{Melanin characterization}

Although the detailed molecular structure of melanin is poorly understood, the term melanin is used for black or brown polymeric pigments that are acid resistant and are bleached by oxidizing agents (Nosanchuk, Stark, \& Casadevall, 2015); the pigment from I. obliquus in this study had these characteristics. It is generally accepted that melanin is insoluble except in highly alkaline solutions (Eisenman \& Casadevall, 2012), and it was therefore unexpected that the isolated pigment from the $\mathrm{IO}_{\mathrm{I}}-\mathrm{W}$ extract was soluble in distilled water, even after removal of free carbohydrates by hydrochloric acid treatment to allow precipitation of the pigment $\left(\mathrm{HCl}, 10 \mathrm{mM}, 30 \mathrm{~min}, 20{ }^{\circ} \mathrm{C}\right)$. We therefore investigated if the water-solubility could be due to carbohydrates still present in the melanin fraction. Gas chromatographic analyses after acid methanolysis and derivatization revealed that the fraction still contained a minor quantity of polysaccharides $(\sim 5 \%)$ consisting of glucose, galactose, xylose, galacturonic acid and mannose in a 5:2:2:2:1 ratio, which was in accordance with polysaccharides previously isolated from the same fungal material (Wold et al., 2018). When the melanin fraction was treated for an extended time with a stronger $\mathrm{HCl}$ solution $\left(6 \mathrm{M}, 12 \mathrm{~h}, 100{ }^{\circ} \mathrm{C}\right.$ ), the pigment became insoluble in distilled water and was completely devoid of carbohydrates, suggesting that the sugars were covalently bound to the melanin polymer and were crucial for the water-solubility of the pigment. The watersolubility of the melanin fraction is an interesting characteristic, since $I$. obliquus is traditionally consumed as a water decoction to treat gastrointestinal disorders and therefore contains large amounts of these pigments (Shikov et al., 2014). As such, the interaction between $I$. obliquus melanin, gut microbiota and the intestinal immune system should be explored in order to understand the mechanisms behind the potential health benefits of this fungus. It has been suggested that the majority of melanin-producing fungi synthesize melanin by one of two pathways based on the precursor molecules utilized (Eisenman \& Casadevall, 2012). One pathway uses L-3,4-dihydroxyphenylalanine (LDOPA) or similar precursors to give a pigment known as eumelanin, whereas in the other pathway, 1,8-dihydroxynaphthalene (DHN) or similar precursors polymerize into allomelanin, also known as DHNmelanin or polyketide melanin. To determine the type of melanin found in I. obliquus, combustion analysis of the water-soluble melanin fraction and the acid-treated insoluble melanin was performed. The results showed a complete absence of nitrogen in both samples, in contrast to the control, an eumelanin from Sepia officinalis, which had a C:N ratio of approximately 7:1 (Magarelli, Passamonti, \& Renieri, 2010). The absence of nitrogen suggests that I. obliquus contains DHN-type melanin (Fig. 2). A polyketide synthase gene putatively responsible for melanin synthesis in I. obliquus was recently reported, further strengthening this hypothesis (Xu, Zhao, \& Zheng, 2015). Further, another paper comparing alkaline-extracted melanin from wild and cultivated I. obliquus (Kukulyanskaya, Kurchenko, Kurchenko, \& Babitskaya, 2002), reported similar findings when analyzing the wild-growing fungus, although the pigment from the cultivated fungal mass contained significant amounts of nitrogen. In a recent paper (Ribera et al., 2019), several fungal strains including $I$. obliquus were investigated for their ability to produce large amounts of melanin. I. obliquus was one of the least efficient melanin producers, but the authors used L-tyrosine - a known eumelanin precursor - to induce melanin production, and this could explain the low levels of melanin production in I. obliquus compared to the other fungi. Here, we measured the approximate polymer size of the melanin fraction to be $<10 \mathrm{kDa}$ by size-exclusion chromatography. This analysis gave a single peak at retention time $\left(R_{t}\right)=22.5$ min while dextran standards used for comparison had $R_{t}$ values ranging from $12.2 \mathrm{~min}$ for the $475 \mathrm{kDa}$ dextran to $20.0 \mathrm{~min}$ for the $11.7 \mathrm{kDa}$ dextran (Figs. S6 and S7). The molecular weights of dextran standards have previously been compared with globular proteins such as albumin by using gel permeation chromatography, giving approximate molecular weight deviations of $10-25 \%$ (Frigon, Leypoldt, Uyejl, \& Henderson, 1983). However, since dextran standards have not previously been used for determining the molecular weight of melanin, the results should be interpreted with caution because the dextran standards are $(1,6)$-linked $\alpha$-glucans with a different three-dimensional shape than melanin. Nevertheless, the fact that the melanin fraction eluted at $>2$ min later than the smallest dextran standard suggests a polymer size $<10 \mathrm{kDa}$. The FT-IR spectrum of the melanin fraction revealed a band at $3500-3100 \mathrm{~cm}^{-1}$, characteristic of hydrogen bonding -OH groups (Fig. S8). Another broad band at $1700-1550 \mathrm{~cm}^{-1}$ could be due to carbon-oxygen double bonds such as in carboxyl groups. These absorption bands were also present in the FT-IR spectrum of Sepia melanin (Fig. S9), and were also in agreement with previous findings (Kukulyanskaya et al., 2002).

In addition to analyzing the intact melanin fraction, degradation products were obtained by treating the acid-treated, carbohydrate-free, water-insoluble melanin with $\mathrm{H}_{2} \mathrm{O}_{2}$ under alkaline conditions. This method has been used by others to identify the presence of eumelanin 


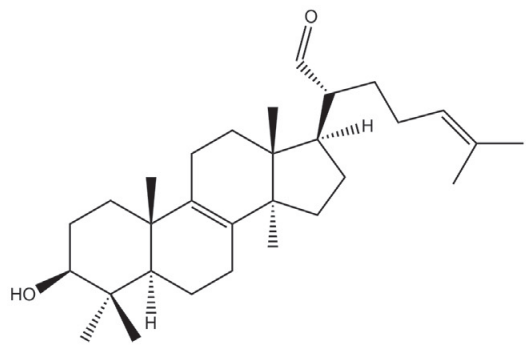

1

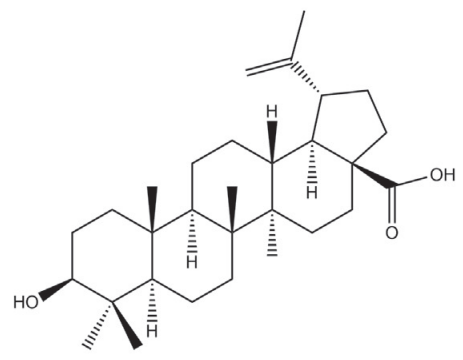

3

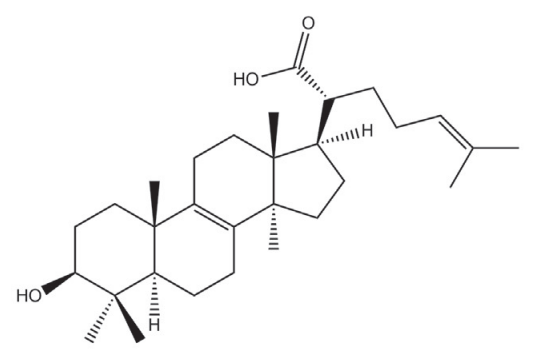

5<smiles>CC(C)=CC[C@@H](O)[C@@H](C)[C@]1(C)CC[C@]2(C)C3=C(CC[C@]21C)[C@@]1(C)CC[C@@H](O)C(C)(C)[C@@]1(C)CC3</smiles>

2

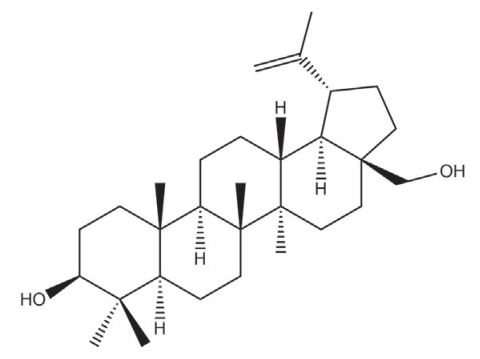

4

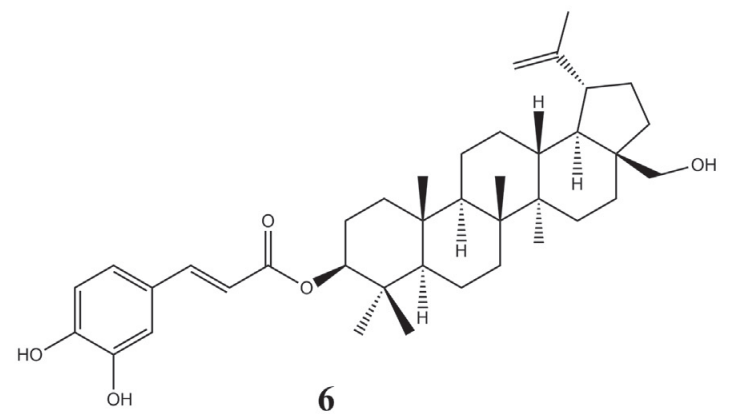

Fig. 1. Compounds 1-6 isolated from I. obliquus.

in biological samples by looking for the well-established eumelanin markers pyrrole-2,3,5-tricarboxylic acid (PTCA) and pyrrole-2,3-dicarboxylic acid (PDCA) in the degradation mixture using reverse phase HPLC (S. Ito et al., 2011). The markers were completely absent from the I. obliquus degradation mixture, while PTCA was the main product in the degradation mixture of eumelanin obtained from $S$. officinalis (Figs. S10-S16). The HPLC chromatogram obtained from the I. obliquus degradation mixture of the melanin fraction revealed a complex mixture of products with high polarity, with a prominent peak at $R_{t} 4.3 \mathrm{~min}$ along with multiple minor signals. Although we were not able to isolate any of the degradation products, the mixture was analyzed by NMR spectroscopy and compared to NMR signals from the melanin fraction itself (Figs. S17-S19). The ${ }^{1} \mathrm{H}$ NMR spectrum of the degradation mixture possessed multiple signals throughout the spectrum between 2 and<smiles>Oc1cccc2cccc(O)c12</smiles>

polymerization

\section{1,8 dihydroxynaphthalene (DHN)}

Water-soluble DHN-type melanin $(<10 \mathrm{kDa})$

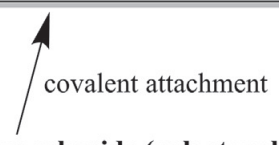

Fungal polysaccharide (galactoxyloglucan)

Fig. 2. Possible scheme of melanin polymerization in Inonotus obliquus. 1,8 dihydroxynaphthalene (DHN) or similar monomeric units polymerize and covalently bind to polysaccharide chains, which make the polymer water-soluble. 
$9 \mathrm{ppm}$. Several minor signals could be observed in the aromatic region (6.0-7.5 ppm), as well as several signals at 3.5-3.9 ppm, which could come from aromatic methoxy-groups. However, the aromatic signals could not be confirmed by ${ }^{13} \mathrm{C}$ NMR or HSQC spectra, possibly due to lower sensitivity in these experiments. The ${ }^{13} \mathrm{C}$ NMR spectrum revealed several resonances at 163-176 ppm, which could come from carboxyl or ester groups. Further, NMR spectra of the water-insoluble melanin (dissolved in $0.2 \mathrm{M} \mathrm{NaOH}$ in $\mathrm{D}_{2} \mathrm{O}$ ) were collected (Figs. S20-S22). Several sharp singlet resonances were observed in both the ${ }^{1} \mathrm{H}$ and ${ }^{13} \mathrm{C}$ NMR spectra that could come from aromatic protons, methoxy groups and carboxylic acids. However, the signals could not be confirmed using HSQC or other 2D experiments, although the HMBC spectrum gave a correlation between a proton at $3.84 \mathrm{ppm}$ and a carbon atom at $150 \mathrm{ppm}$, which could come from methoxy protons coupling with an aromatic carbon. The presence of carboxylic acid groups has to our knowledge not been reported for DHN-type melanin before, although it is found in abundance in eumelanin (S. Ito, Wakamatsu, \& Ozeki, 1993). Since both types of melanin have the common feature of $\mathrm{pH}$ dependent solubility in aqueous solutions, it should be expected that some acidic groups, such as a carboxylic acid, are present in DHN-type melanin pigments as well. This is consistent with the NMR spectra obtained for the melanin isolated from I. obliquus. A comparison of the chemical characteristic between melanin from I. obliquus and S. officinalis is summarized in Table 1 . However, due to the complex nature of melanin combined with the lack of sufficient chemical evidence regarding its detailed structure, it is beyond the scope of this paper to further describe the molecular structure of the melanin pigment from $I$. obliquus.

\subsection{Activation and inhibition of human complement}

Complement is an integral part of the human immune system and functions as a bridge between innate and adaptive immunity. Dysregulation of the complement system has been linked to the development of a wide range of diseases and pathological conditions such as cancer (Ricklin, Reis, \& Lambris, 2016). As such, compounds that can modulate the activity of complement could represent new types of pharmacotherapies. As seen in Fig. 3a, compounds 1, 2 and the melanin fraction interacted strongly with human complement, inhibiting complement-induced hemolysis of sheep erythrocytes with 50\% inhibitory concentration of hemolysis $\left(\mathrm{ICH}_{50}\right)$ values of $0.8 \mu \mathrm{g} / \mathrm{mL} \pm 0.2$ $(1.8 \pm 0.4 \mu \mathrm{M}), 6.6 \pm 1.7 \mu \mathrm{g} / \mathrm{mL}(14.9 \pm 3.9 \mu \mathrm{M})$ and $4.9 \pm 1.1 \mu \mathrm{g} / \mathrm{mL}$, respectively. For the initial screening to detect complement-modulating activity, an incubation period of $30 \mathrm{~min}$ of the compounds with serum (before adding erythrocytes) was used. This time point makes it possible to detect both complement activating and inhibiting activity, but does not discriminate between the two (Inngjerdingen et al., 2006). Compounds 3-6 were inactive in this assay, having $\mathrm{ICH}_{50}$ values $>100 \mu \mathrm{g} / \mathrm{mL}$ (Table 2, $30 \mathrm{~min}$ incubation). The difference in activities of 3,4 and 6 could be because they have a different triterpene skeleton than $\mathbf{1}$ and $\mathbf{2}$, with a pentacyclic backbone instead of a tetracyclic one. However, compound $\mathbf{5}$ shares the same backbone as $\mathbf{1}$ and $\mathbf{2}$ but was also inactive; in this case it could be due to a difference in polarity. Therefore, it would be necessary to evaluate a larger library of similar types of triterpenes in this assay in order to identify the pharmacophores of $\mathbf{1}$ and $\mathbf{2}$.
To determine if the interaction was due to inhibition or activation of complement, compounds $\mathbf{1}$ and $\mathbf{2}$ and the melanin fraction were incubated with serum for either 0 min or $30 \mathrm{~min}$ before erythrocytes were added. Full activation of complement prior to hemolysis requires some time whereas inhibition of complement components can take effect almost immediately.(Alban et al., 2002) Therefore, compounds that act as inhibitors of complement should have similar $\mathrm{ICH}_{50}$ values at different incubation times, whereas activators should appear more active over time. As seen from Table 2, the activity of $\mathbf{1}$ was almost 20 -fold lower at $0 \mathrm{~min}$ incubation when compared to $30 \mathrm{~min}$ incubation $\left(\mathrm{ICH}_{50}\right.$ value of $14.7 \pm 2.3 \mu \mathrm{g} / \mathrm{mL}$ vs $0.8 \pm 0.2 \mu \mathrm{g} / \mathrm{mL}$ ), while 2 became completely inactive $\left(\mathrm{ICH}_{50}\right.$ value $>50 \mu \mathrm{g} / \mathrm{mL}$ vs $6.6 \pm 1.7 \mu \mathrm{g} / \mathrm{mL}$ for $0 \mathrm{~min}$ and $30 \mathrm{~min}$ incubation, respectively). This suggests that these two compounds are activators of complement, but the results for $\mathbf{1}$ should be interpreted with caution because this compound still showed significant activity even at $0 \mathrm{~min}$. The melanin fraction had comparable activity regardless of incubation time $\left(\mathrm{ICH}_{50}\right.$ values of $8.4 \pm 0.1 \mu \mathrm{g} /$ $\mathrm{mL}$ and $4.9 \pm 1.1 \mu \mathrm{g} / \mathrm{mL}$ for $0 \mathrm{~min}$ and $30 \mathrm{~min}$ incubation, respectively), suggesting an inhibitory effect. This was also the case for the positive control heparin, which is a known inhibitor of the classical and alternative pathways of complement (Alban et al., 2002). To further explore how the compounds interacted with complement, we used a pathway specific ELISA kit originally developed to detect deficiencies in the complement cascade in clinical serum samples (SvarLifeScience, 2015). As shown in Fig. 3b, compounds 1 and $\mathbf{2}$ interacted through the classical pathway (52.8\% and $39.7 \%$ of total, respectively) and lectin pathway (44.6\% and $55 \%$ of total), with almost no detectable interaction with the alternative pathway. In contrast, the melanin fraction interacted with all three pathways to a similar extent (33.4\%, 34.8\% and $31.8 \%$ for the classical, lectin and alternative pathways, respectively). Although the classical and lectin pathways are usually triggered by distinct mechanisms (antibody-mediated vs. carbohydrate-mediated activation), they use some of the same complement components early in the complement cascade, in particular C2 and C4, which are not used in the alternative pathway (Ricklin et al., 2016). Since 1 and 2 did not interact with the alternative pathway, it is likely that they interact in some way with C2 and/or C4 as suggested by the manufacturer of the ELISA kit (SvarLifeScience, , 2015). As the melanin fraction interacted with all three complement pathways, it suggests that the inhibition occurs at a late stage in the cascade, inhibiting either C3 cleavage or one of the components C5-C9 (SvarLifeScience, , 2015). However, not much literature exists on the interaction of triterpenoids or melanin with human complement. In one study (Seo et al., 2009), they found that gandoderic acid isolated from Ganoderma lucidum had a weak inhibitory effect on complement activity through the classical pathway (IC 50 value of $44.6 \mu \mathrm{M}$ ). In another study (Rosas, MacGill, Nosanchuk, Kozel, \& Casadevall, 2002), fungal melanin "ghost" particles produced by Cryptococcus neoformans and Aspergillus niger were found to activate the alternative pathway of human complement via binding of C3 fragments. The type of melanin tested was not stated, but it has been reported that $C$. neoformans possesses the eumelanin type (Nosanchuk et al., 2015). To our knowledge, this is the first report of complement interacting compounds to be isolated from I. obliquus, and the potent activity of some of these demonstrates the immunomodulatory potential of this fungus. This seems to be particularly true for compound 1, which in addition to being the most abundant triterpenoid in the extract

Table 1

Comparison of the chemical properties of melanin from I. obliquus and S. officinalis.

\begin{tabular}{|c|c|c|c|c|c|c|c|c|}
\hline & Color & $\begin{array}{l}\text { Resistant to acid } \\
\text { degradation }\end{array}$ & $\begin{array}{l}\text { Bleached by } \\
\mathrm{H}_{2} \mathrm{O}_{2}\end{array}$ & $\begin{array}{l}\text { Soluble in } \\
\text { alkali }\end{array}$ & Carbohydrate content & $\mathrm{C}: \mathrm{N}$ ratio & Molecular weight & $\begin{array}{l}\text { Eumelanin markers found (PTCA/ } \\
\text { PDCA) }\end{array}$ \\
\hline I. obliquus & Black & + & + & + & $5 \%$ & n.d. & $<10 \mathrm{kDa}$ & - \\
\hline S. officinalis & Black & + & + & + & - & $7: 1$ & n.c.* & + \\
\hline
\end{tabular}

n.d. = nitrogen $(\mathrm{N})$ was not detected. n.c. = not calculated due to insolubility in the buffer used for HPLC analysis. 
a)

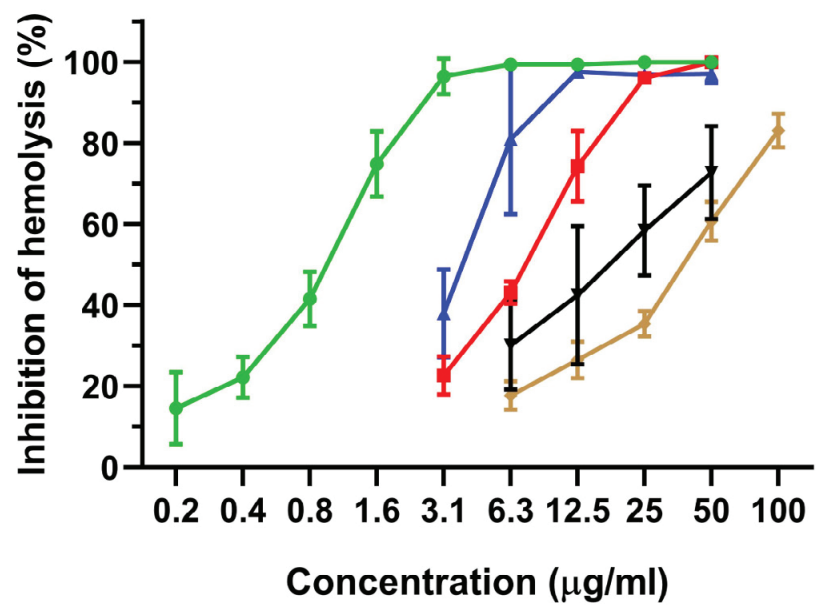

b)

Interaction with complement pathways

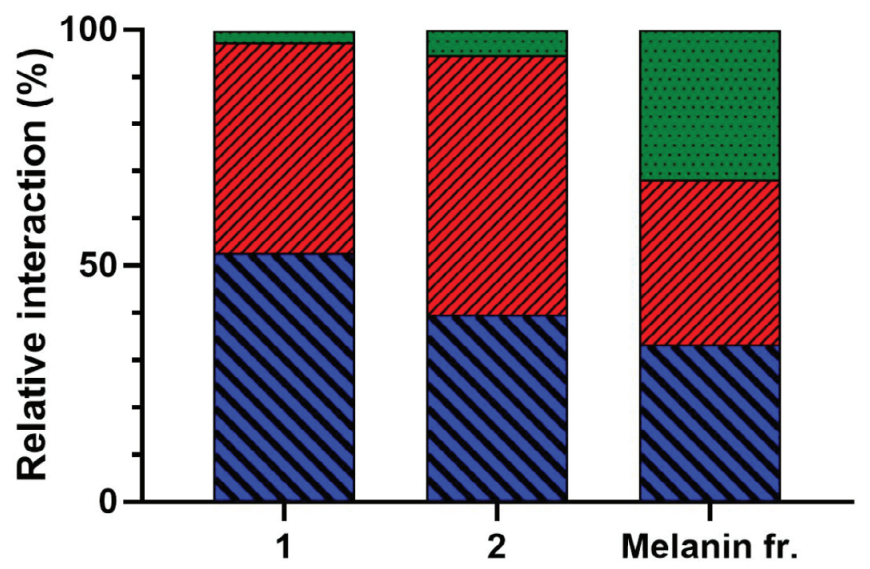

Table 2

Inhibition of serum-induced hemolysis of sheep erythrocytes.

\begin{tabular}{lll}
\hline Compound & $\begin{array}{l}\text { ICH} \\
\text { incubation }\end{array}$ & $\begin{array}{l}\mathrm{ICH}_{50} \text { values }(\mu \mathrm{g} / \mathrm{mL}), 30 \mathrm{~min} \\
\text { incubation }\end{array}$ \\
\hline $\mathbf{1}$ & $14.7 \pm 2.3$ & $0.8 \pm 0.2$ \\
$\mathbf{2}$ & $>50$ & $6.6 \pm 1.7$ \\
$\mathbf{3}$ & n.t. & $>100$ \\
$\mathbf{4}$ & n.t. & $>100$ \\
$\mathbf{5}$ & n.t. & $>100$ \\
$\mathbf{6}$ & n.t. & $>100$ \\
Melanin fraction & $8.4 \pm 0.1$ & $4.9 \pm 1.1$ \\
BP II & n.t. & $27.7 \pm 6.5$ \\
Heparin & $42.6 \pm 3$ & $40.7 \pm 4.2$ \\
\hline
\end{tabular}

BP-II (a polysaccharide isolated from Biophytum petersianum) and heparin were used as positive controls. Results are presented as means \pm SD $(n=3)$. n.t $=$ not tested

( $\sim 1.6 \%$ of the $\mathrm{IO}_{\mathrm{I}}$-DCM extract) was also the most active of the compounds. Additionally, there are no reports in the literature that triterpene aldehyde $\mathbf{1}$ has been isolated from any other sources than $I$. obliquus.
Fig. 3. (a) Inhibition of complement-induced hemolysis of sheep erythrocytes. Compounds were incubated with human serum for 30 min prior to addition of erythrocytes. BPII (a polysaccharide isolated from $B$. petersianum) and heparin were used as positive controls. Results are expressed as means $\pm S D(n=3)$. (b) Interaction of compounds 1,2 and the melanin fraction (fr.) with the classical, lectin and alternative pathways of the complement cascade. Results are expressed as percentage interaction with each pathway, the total interaction with all pathways was set to $100 \%$. The results are expressed as averages of compound interaction with two different human sera.

\section{A.:- Alternative \\ שn] Lectin \\ D. Classical}

\subsection{Anti-inflammatory activity in LPS + IFN $\gamma$-activated macrophages}

Macrophages can be activated into a pro-inflammatory phenotype by LPS and IFN $\gamma$. Upon activation, macrophages release mediators such as cytokines and nitric oxide (NO) (Bogdan, 2015). NO is involved in a variety of physiological processes, and functions as an important signaling and effector molecule in the immune system. However, if produced in excess, NO can potentially cause chronic inflammation and autoimmune diseases (Bogdan, 2015). Compounds that inhibit NO produced by macrophages are therefore anti-inflammatory, and might play a role in prevention and treatment of certain inflammatory diseases. All isolated compounds from I. obliquus were tested for their ability to reduce LPS + IFN $\gamma$-induced NO production in murine primary macrophages. In addition, an MTT assay was carried out subsequently to determine how the compounds affected the viability of the macrophages. As shown in Fig. 4, compound 6 and the melanin fraction gave a significant reduction in NO levels without affecting cell viability, with $\mathrm{IC}_{50}$ values of $10.6 \pm 2.2 \mu \mathrm{g} / \mathrm{mL}(17.6 \pm 3.7 \mu \mathrm{M})$ and $24.1 \pm 7.9 \mu \mathrm{g} / \mathrm{mL}$, respectively. Compounds $\mathbf{1}$ and $\mathbf{5}$ also appeared to inhibit NO in a dose-dependent manner, but the results were inversely correlated with cell viability, implying a cytotoxic or cytostatic effect on the cells that could have affected NO production. Compounds 2, 3 and 4 greatly reduced viability in all concentrations tested (results not 
a)



c)

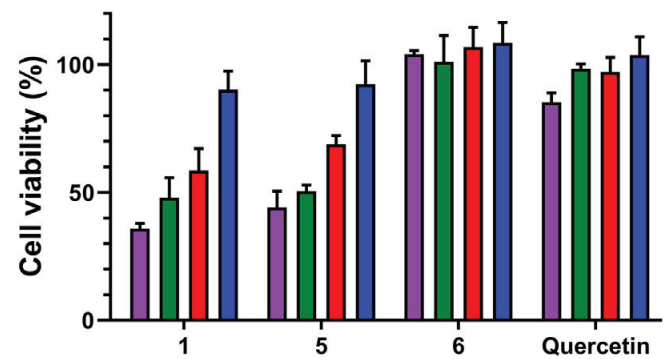

b)

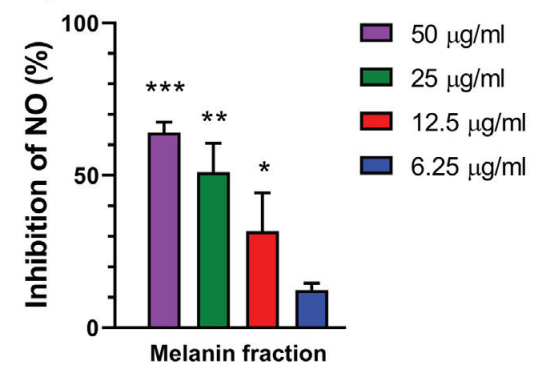

d)

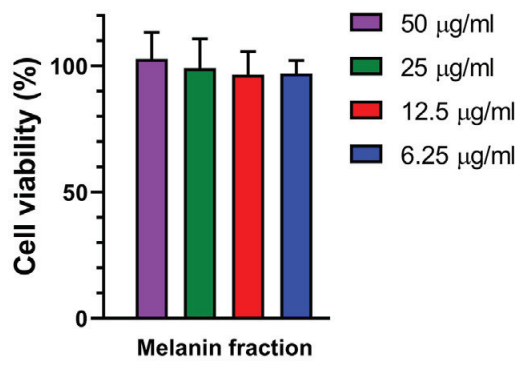

Fig. 4. Anti-inflammatory effects and cell viability in murine BL6 macrophages by 1, 5, 6 and the melanin fraction. Quercetin was used as a positive control. (a, b) Inhibition of LPS-induced NO production, presented as percent inhibition of NO compared to LPS-treated cells. Results are expressed as means \pm SD ( $\mathrm{n}=3$ ). $* * *=$ $\mathrm{p}<0.001,{ }^{*}=\mathrm{p}<0.01,{ }^{*}=\mathrm{p}<0.05$, compared to control cells. Statistical significance for NO inhibition is adjusted against cell viability. (c, d) Viability of macrophages, measured subsequently to the Griess assay, expressed as means $\pm \operatorname{SD}(n=3)$.

shown). Compounds 1, 2 and 5 have previously been investigated for their anti-inflammatory effects in a similar setup as described here (Ma, Chen, Dong, \& Lu, 2013). The authors found a weak anti-inflammatory effect of 5, whereas the other sterols and triterpenoids tested were inactive. However, concentrations of the compounds were not stated, and the lack of a subsequent viability assay makes these results less reliable. To our knowledge, this is the first time compound 6 and I. obliquus melanin have been investigated for their anti-inflammatory effects. Interestingly, it appears that the presence of the 3-O-caffeate moiety, which is the difference between compounds 4 and 6 , removes the cytotoxic effects while increasing the anti-inflammatory properties of the compound. In another study (Jeong et al., 2009), it was found that betulinic acid 3-O-caffeate, but not ursolic acid 3-O-caffeate, inhibited NO production in RAW 264.7 cells. Taken together, these findings suggest that both the triterpenoid part and the caffeic acid moiety in compounds similar to 6 are important for their anti-inflammatory effect.

\subsection{Cell viability in human cancer cell lines}

All compounds were screened for their ability to reduce cell viability of the two human cancer cell lines NCI-H460 (lung carcinoma) and HT29-MTX (methotrexate-resistant colon adenocarcinoma), using the MTT assay. To our knowledge, this is the first time compounds 1-6 have been tested on HT29-MTX cells. As shown in Table 3, compounds 2,3 and 4 reduced viability in both cells lines after $24 \mathrm{~h}$, with $\mathrm{IC}_{50}$ values $<5 \mu \mathrm{M}$. The other compounds were inactive with $\mathrm{IC}_{50}$ values $>50 \mu \mathrm{M}$ in both cell lines. It is well established that 2, 3 and 4 are cytotoxic to cancer cells by triggering apoptosis via caspase-dependent mechanisms, although detailed mechanisms of action are not completely understood (Kommera, Kaluderovic, Kalbitz, \& Paschke, 2011; Nomura, Takahashi, Uesugi, Tanaka, \& Kobayashi, 2008). As seen from Fig. 5, the results from the NCI-H460 cell line are in accordance with apoptotic cell death, giving a clear dose-dependent effect of 2, 3 and 4 and indicating that they are cytotoxic. For the HT29-MTX cell line, the cell viability compared to untreated cells seemed to stabilize at 30-40\%
Table 3

Viability of human cancer cell lines NCI-H460 and HT29-MTX after $24 \mathrm{~h}$ treatment with the isolated I. obliquus metabolites.

\begin{tabular}{lcc}
\hline Compound & NCI-H460 & HT29-MTX \\
\hline $\mathbf{1}$ & $33 \pm 6 \mu \mathrm{M}$ & $>50 \mu \mathrm{M}$ \\
$\mathbf{2}$ & $4.7 \pm 1 \mu \mathrm{M}$ & $3.8 \pm 0.8 \mu \mathrm{M}$ \\
$\mathbf{3}$ & $2.1 \pm 0.5 \mu \mathrm{M}$ & $0.8 \pm 0.3 \mu \mathrm{M}$ \\
$\mathbf{4}$ & $2.8 \pm 0.4 \mu \mathrm{M}$ & $1.6 \pm 0.4 \mu \mathrm{M}$ \\
$\mathbf{5}$ & $>50 \mu \mathrm{M}$ & $>50 \mu \mathrm{M}$ \\
$\mathbf{6}$ & $>50 \mu \mathrm{M}$ & $>50 \mu \mathrm{M}$ \\
Melanin fraction & $>50 \mu \mathrm{g} / \mathrm{mL}$ & $>50 \mu \mathrm{g} / \mathrm{mL}$ \\
Podophyllotoxin & $22 \pm 6 \mu \mathrm{M}$ & $24 \pm 3 \mu \mathrm{M}$ \\
\hline
\end{tabular}

$\mathrm{IC}_{50}$ values are given as $50 \%$ reduction in cell viability compared to untreated cells, and are presented as means $\pm \mathrm{SD}(\mathrm{n}=3)$.

even at higher concentrations (up to $100 \mu \mathrm{M}$ ), indicating a cytostatic rather than cytotoxic effect, in contrast to the NCI-H460 cells where viability was reduced to below $5 \%$ at $100 \mu \mathrm{M}$ (data not shown).

However, based on $\mathrm{IC}_{50}$ values, compounds 2, 3 and 4 appear more potent against HT29-MTX cells than against NCI-H460 cells. It has previously been reported (Kvasnica, Sarek, Klinotova, Dzubak, \& Hajduch, 2005) that compound 3 is only mildly cytotoxic to HT29 cells $\left(\mathrm{IC}_{50}\right.$ value $\left.=84.5 \mu \mathrm{M}\right)$ while 4 is inactive $\left(\mathrm{IC}_{50}\right.$ value $\left.>250 \mu \mathrm{M}\right)$, in contrast to the findings presented here. As HT29-MTX cells are resistant to the chemotherapeutic drug methotrexate, it could be that they respond differently to these compounds than non-drug resistant HT29 cells, and these discrepancies should be explored further. However, Compounds 2-4 are present in EtOH extracts from I. obliquus (Zhao et al., 2015), and such extracts have previously been reported to have cytotoxic effects against colon cancer cells (Tao, Li, Li, \& Li, 2018). The ability of these compounds to reduce viability of human colon cancer cell lines such as HT29-MTX at low concentrations may suggest that extracts containing these compounds could have a preventative effect on the development of intestinal and colorectal cancers in vivo as well, despite or perhaps because of their poor bioavailability in humans. 
a)

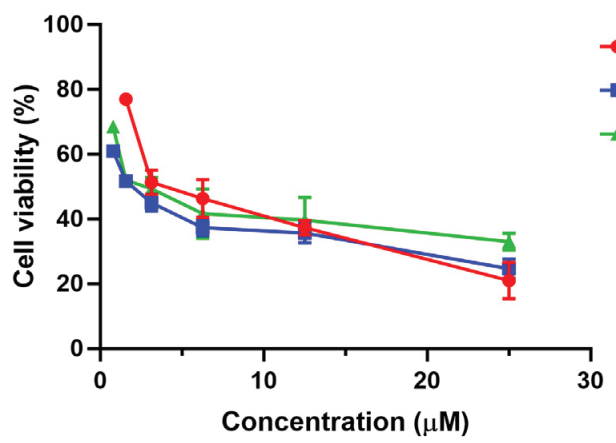

b)

\section{HT29-MTX}

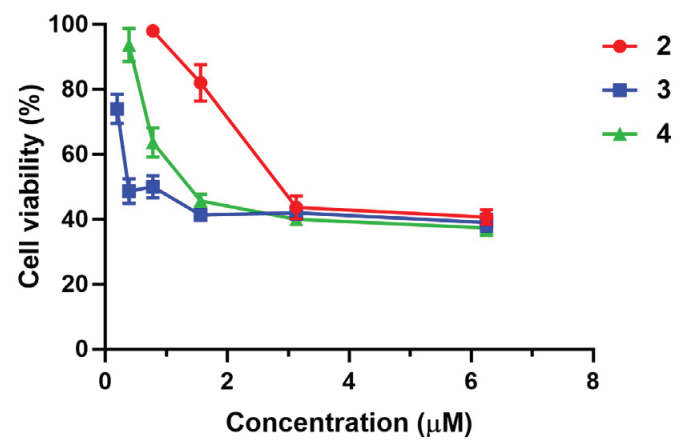

Fig. 5. Cell viability of (a) NCI-H460 cells and (b) HT29-MTX cells, after treatment with compounds 2,3 or 4 for 24 h. Results are expressed as means \pm SD (n $=3$ ).

\subsection{Inhibition of xanthine oxidase}

All the isolated $I$. obliquus compounds were inactive in a $\mathrm{XO}$ inhibition assay $\left(\mathrm{IC}_{50}\right.$ value $\left.>164 \mu \mathrm{M}\right)$. Yong et al. recently reported that an EtOH extract from I. obliquus was able to inhibit XO activity in mice, causing a significant reduction in serum uric acid levels (Yong et al., 2018). Interestingly, it was suggested that the XO inhibiting effect was due to triterpenoids such as $\mathbf{4}$ and $\mathbf{5}$, which according to in silico molecular docking simulations blocked the substrate site of XO. In addition, it was stated that compound $\mathbf{5}$ gave in vitro inhibition of XO comparable to allopurinol, albeit with quite high $\mathrm{IC}_{50}$ values ( $>100 \mu \mathrm{M}$ ). However, both compounds 4 and 5 were inactive in the screening presented here, whereas allopurinol had an $\mathrm{IC}_{50}$ value of $0.4 \pm 0.1 \mu \mathrm{M}$. To explore these discrepancies, we tested the $\mathrm{IO}_{\mathrm{I}}-\mathrm{DCM}$ and $\mathrm{IO}_{\mathrm{I}}-\mathrm{MeOH}$ extracts for XO inhibitory activity. $\mathrm{IO}_{\mathrm{I}}-\mathrm{DCM}$ was found inactive $\left(\mathrm{IC}_{50}>164 \mu \mathrm{g} / \mathrm{mL}\right.$ ) while $\mathrm{IO}_{\mathrm{I}}-\mathrm{MeOH}$ inhibited $\mathrm{XO}\left(\mathrm{IC}_{50}\right.$ value of $20.5 \pm 0.5 \mu \mathrm{g} / \mathrm{mL}$ ). Based on the ${ }^{1} \mathrm{H}$ NMR spectra for these two extracts, $\mathrm{IO}_{\mathrm{I}}-\mathrm{DCM}$ contains mainly triterpenoids, whereas $\mathrm{IO}_{\mathrm{I}}-\mathrm{MeOH}$ does not (Figs. S3 and S4). $\mathrm{MeOH}$ was used for extraction subsequently to DCM extraction in our scheme, and this is probably the main reason for the lack for terpenoids in the $\mathrm{MeOH}$ extract. To explore further, we extracted I. obliquus directly with $\mathrm{EtOH}$, and then fractionated the extract using $100 \% \mathrm{MeOH}$ (fraction A), 50/50\% $\mathrm{MeOH} / \mathrm{H}_{2} \mathrm{O}$ (fraction B) and $100 \% \mathrm{H}_{2} \mathrm{O}$ (fraction $\mathrm{C}$ ) using a C13-column. The EtOH extract (IC $\mathrm{I}_{50}$ value of $33.6 \pm 9.9 \mu \mathrm{g} / \mathrm{mL}$ ) had similar $\mathrm{XO}$ inhibiting activity to our $\mathrm{IO}_{\mathrm{I}}-\mathrm{MeOH}$ extract. Fraction $\mathrm{A}$ and $\mathrm{C}$ from the EtOH extract were inactive against XO ( $\mathrm{IC}_{50}$ values $>100 \mu \mathrm{g} / \mathrm{mL}$ ) while fraction B was highly active $\left(\mathrm{IC}_{50}\right.$ value $=2.8 \pm 0.1 \mu \mathrm{g} / \mathrm{mL}$ ). The ${ }^{1} \mathrm{H}$ NMR spectrum of fraction A revealed the presence of sharp methyl singlets at 0.7-2.0 ppm indicative of triterpenoids, and these signals were absent in fractions B and C (Figs. S23-S25), suggesting that the triterpenoids were not responsible for the XO inhibiting effect. The EtOH extract used by Yong et al. should be expected to contain triterpenoids due to the direct extraction of fungal material using EtOH as a solvent, possibly leading the authors to the faulty conclusion that triterpenoids are the active compounds responsible for the XO inhibiting effect. Further effort is therefore necessary to identify the I. obliquus compounds responsible for the observed XO inhibiting effects.

\subsection{Inhibition of 15-lipoxygenase and acetylcholinesterase}

None of the isolated $I$. obliquus compounds showed inhibitory activity against soybean 15-LOX ( IC $_{50}$ values $\left.>164 \mu \mathrm{M}\right)$. However, compound 6 was able to slightly inhibit the enzymatic activity at all concentrations tested, ranging from $\sim 45 \%$ inhibition at $164 \mu \mathrm{M}$ to $\sim 20 \%$ inhibition at $12.5 \mu \mathrm{M}$. In contrast, the positive control quercetin gave complete inhibition of 15-LOX at high concentrations ( $>90 \%$ inhibition at $328 \mu \mathrm{M}$ ), but lost most activity at lower concentrations $(<10 \%$ inhibition at $83 \mu \mathrm{M})$. This indicates that 6 may act as a partial inhibitor of 15-LOX, reducing - but not completely blocking - the activity of the enzyme even at lower concentrations. None of the compounds showed any inhibitory activity against Electrophorus electricus acetylcholinesterase.

\subsection{Antimicrobial activity}

The antimicrobial activity of the isolated I. obliquus compounds was investigated using the disk diffusion test, using four bacterial strains ( $E$. coli, $S$. aureus, B. subtilis, $P$. aeruginosa) and one fungal strain (C. albicans). The isolated compounds were not able to inhibit the growth of any of the strains tested, while the $\mathrm{IO}_{\mathrm{I}}$-DCM extract was slightly active against $S$. aureus, with inhibiting effects seen at $250 \mu \mathrm{g} / \mathrm{disc}$ (inhibition zone $=0.5 \mathrm{~cm}$ ). The fact that the melanin fraction did not have any anti-microbial activity even at high concentrations against gut-associated bacteria like $E$. coli and B. subtilis is interesting because I. obliquus is traditionally consumed as a melanin-containing water decoction, which potentially could interact with such bacteria. Further investigation into melanin's effect on gut-associated bacteria is warranted to understand the role of $I$. obliquus decoctions against gastrointestinal disorders.

\subsection{DPPH radical scavenging activity}

Compound 6 and the melanin fraction were able to scavenge DPPH with $\mathrm{IC}_{50}$ values of $31.5 \pm 1.6 \mu \mathrm{g} / \mathrm{mL}(52 \pm 2.7 \mu \mathrm{M})$ and $61.4 \mu \mathrm{g} / \mathrm{mL}$, respectively. The other $I$. obliquus compounds had no radical scavenging activity in this assay.

\section{Conclusion}

Six triterpenoids and a melanin fraction from the increasingly consumed fungus $I$. obliquus were isolated and chemically characterized, and investigated for their anti-inflammatory, immunological and cytotoxic activities using various bioassays. The melanin fraction was found to be of the DHN-type, and was covalently bound to polysaccharides that gave the pigment high solubility in aqueous solutions. The melanin fraction, as well as the triterpenoids $3 \beta$-hydroxy-8,24dien-21-al (1) and inotodiol (2) displayed potent activity in a human complement protein fixation assay. Compound $\mathbf{1}$ and $\mathbf{2}$ activated the complement cascade, whereas the melanin fraction inhibited the same cascade. Compound 2, as well as betulinic acid (3) and betulin (4) had anti-proliferative properties against the methotrexate-resistant human adenocarcinoma cell line HT29-MTX and the human lung carcinoma cell line NCI-H460. Further, the melanin fraction and betulin-3-Ocaffeate (compound 6 , reported for the first time from $I$. obliquus) were able to reduce NO production in primary murine macrophages. None of 
the metabolites displayed toxicity against bacteria commonly present in the human gut microbiota such as E. coli and B. subtilis. Taken together, the results demonstrate the anti-inflammatory and immunomodulatory effects of I. obliquus melanin and triterpenoids. As a significant portion of the human immune system is located in the gut, it is possible that the immunomodulatory effects described herein are clinically relevant even if the compounds are not absorbed and distributed systemically. Studies should therefore be conducted in order to investigate the in vivo bioavailability and -activity of the isolated metabolites.

\section{Ethics statements file}

The research conducted for this manuscript did not include any human or animal experiments.

\section{Acknowledgements}

This work was partly supported by the Research Council of Norway through the Norwegian NMR platform, NNP (226244/F50). The authors would like to thank Drs. Kazumasa Wakamatsu and Shosuke Ito, Fujita Health University School of Medical Sciences, Japan for the donation of the melanin markers, and professor K. A. Solhaug and C. Kreibich at NMBU for the elemental analysis of the melanin samples. Also, thanks to professor emeritus Karl Malterud for valuable input throughout the project. The authors would like to thank the Norwegian PhD School of Pharmacy, NORAM and UiO:Life Science for funding a 6months research stay at Scripps Institution of Oceanography, University of California San Diego, CA, USA. The picture of the I. obliquus sclerotium in the graphical abstract was used under the Creative Commons licence CC BY-NC-SA 2.0. The picture can be found online through Distant Hill Gardens (https://www.flickr.com/photos/59898141@N06/ 11106545074).

\section{Declaration of Competing Interest}

The authors declare no conflict of interest.

\section{Appendix A. Supplementary data}

Supporting information is available free of charge via the internet, and includes figures of NMR spectra, HRESIMS data, HPLC chromatograms, FT-IR spectra and UV-vis data. Supplementary data to this article can be found online at https://doi.org/10.1016/j.jff.2020.104025.

\section{References}

Alban, S., Classen, B., Brunner, G., \& Blaschek, W. (2002). Differentiation between the complement modulating effects of an Arabinogalactan-protein from Echinacea purpurea and heparin. Planta Medica, 68, 1118-1124.

Bergheim, S., Malterud, K. E., \& Anthonsen, T. (1991). Preparative scale separation of neutral lipids and phospholipids by centrifugally accelerated thin-layer chromatography. Journal of Lipid Research, 32, 877-879.

Bogdan, C. (2015). Nitric oxide synthase in innate and adaptive immunity: An update. Trends in Immunology, 36(3), 161-178. https://doi.org/10.1016/j.it.2015.01.003.

Braunlich, M., Slimestad, R., Wangensteen, H., Brede, C., Malterud, K. E., \& Barsett, H. (2013). Extracts, anthocyanins and procyanidins from Aronia melanocarpa as radical scavengers and enzyme inhibitors. Nutrients, 5(3), 663-678. https://doi.org/10. $3390 /$ nu5030663.

Du, B., Zhu, F., \& Xu, B. (2018). An insight into the anti-inflammatory properties of edible and medicinal mushrooms. Journal of Functional Foods, 47, 334-342. https://doi.org/ 10.1016/j.jff.2018.06.003.

Eisenman, H. C., \& Casadevall, A. (2012). Synthesis and assembly of fungal melanin. Applied Microbiology and Biotechnology, 93(3), 931-940. https://doi.org/10.1007/ s00253-011-3777-2.

Frigon, R. P., Leypoldt, J. K., Uyejl, S., \& Henderson, L. W. (1983). Disparity between Stokes radii of dextrans and proteins as determined by retention volume in gel permeation chromatography. Analytical Chemistry, 55, 1349-1354.

Fuchino, H., Satoh, T., \& Tanaka, N. (1995). Chemical evaluation of Betula species in Japan. I. Constituents of Betula ermanii. Chemical \& Pharmaceutical Bulletin, 43(11), 1937-1942.

Furtado, N. A. J. C., Pirson, L., Edelberg, H. L. M. M., Loira-Pastoriza, C., Preat, V., ...
Andre, C. M. (2017). Pentacyclic triterpene bioavailability: An overview of in vitro and in vivo studies. Molecules, 22(3), https://doi.org/10.3390/molecules22030400.

Herald, T. J., Gadgil, P., \& Tilley, M. (2012). High-throughput micro plate assays for screening flavonoid content and DPPH-scavenging activity in sorghum bran and flour. Journal of the Science of Food and Agriculture, 92(11), 2326-2331. https://doi. org/10.1002/jsfa.5633.

Ho, G. T., Wangensteen, H., \& Barsett, H. (2017). Elderberry and elderflower extracts, phenolic compounds, and metabolites and their effect on complement, RAW 264.7 macrophages and dendritic cells. International Journal of Molecular Sciences, 18(3), https://doi.org/10.3390/ijms18030584.

Inngjerdingen, K. T., Coulibaly, A., Diallo, D., Michaelsen, T. E., \& Paulsen, B. S. (2006). A complement fixing polysaccharide from Biophytum petersianum Klotzsch, a medicinal plant from Mali, West Africa. Biomacromolecules, 7, 48-53.

Ito, S., Nakanishi, Y., Valenzuela, R. K., Brilliant, M. H., Kolbe, L., \& Wakamatsu, K. (2011). Usefulness of alkaline hydrogen peroxide oxidation to analyze eumelanin and pheomelanin in various tissue samples: Application to chemical analysis of human hair melanins. Pigment Cell \& Melanoma Research, 24(4), 605-613. https://doi.org/ 10.1111/j.1755-148X.2011.00864.x.

Ito, S., Wakamatsu, K., \& Ozeki, H. (1993). Spectrophotometric assay of eumelanin in tissue samples. Analytical Biochemistry, 215(2), 273-277.

Jeong, W., Hong, S. S., Kim, N., Yang, Y. T., Shin, Y. S., Lee, C., ... Lee, D. (2009). Bioactive triterpenoids from Callistemon lanceolatus. Archives of Pharmacal Research, 32(6), 845-849. https://doi.org/10.1007/s12272-009-1605-3.

Kahlos, K., Hiltunen, R., \& Schantz, M. (1984). 3ß-hydroxy-lanosta-8,24-dien-21-al, a new triterpene from Inontus obliquus. Planta Medica, 50(2), 197-198.

Kommera, H., Kaluderovic, G. N., Kalbitz, J., \& Paschke, R. (2011). Lupane triterpenoids betulin and betulinic acid derivatives induce apoptosis in tumor cells. Invest New Drugs, 29(2), 266-272. https://doi.org/10.1007/s10637-009-9358-x.

Kukulyanskaya, T. A., Kurchenko, N. V., Kurchenko, V. P., \& Babitskaya, V. G. (2002). Physicochemical properties of melanins produced by the sterile form of Inonotus obliquus ("Chagi") in natural and cultivated fungus. Applied Biochemistry and Microbiology, 38(1), 58-61.

Kvasnica, M., Sarek, J., Klinotova, E., Dzubak, P., \& Hajduch, M. (2005). Synthesis of phthalates of betulinic acid and betulin with cytotoxic activity. Bioorganic \& Medicinal Chemistry, 13(10), 3447-3454. https://doi.org/10.1016/j.bmc.2005.03.006.

Lesuffleur, T., Porchet, N., Aubert, J.-P., Swallow, D., Gum, J. R., Kim, Y. S., ... Zweibaum, A. (1993). Differential expression of the human mucin genes MUC1 to MUC5 in relation to growth and differentiation of different mucus-secreting HT-29 cell subpopulations. Journal of Cell Science, 106, 771-783.

Ma, L., Chen, H., Dong, P., \& Lu, X. (2013). Anti-inflammatory and anticancer activities of extracts and compounds from the mushroom Inonotus obliquus. Food Chemistry, 139(1-4), 503-508. https://doi.org/10.1016/j.foodchem.2013.01.030.

Magarelli, M., Passamonti, P., \& Renieri, C. (2010). Purification, characterization and analysis of sepia melanin from commercial sepia ink (Sepia officinalis). Revista CES Medicina Veterinaria y Zootecnia, 5(2), 18-28.

Muller, E., Christopoulos, P. F., Halder, S., Lunde, A., Beraki, K., Speth, M., ... Corthay, A. (2017). Toll-like receptor ligands and interferon-gamma synergize for induction of antitumor M1 macrophages. Frontiers of Immunology, 8. https://doi.org/10.3389/ fimmu. 2017.01383.

Nomura, M., Takahashi, T., Uesugi, A., Tanaka, R., \& Kobayashi, S. (2008). Inotodiol, a lanostane triterpenoid, from Inonotus obliquus inhibits cell proliferation through caspase-3-dependent apoptosis. Anticancer Research, 28, 2691-2696.

Nosanchuk, J. D., Stark, R. E., \& Casadevall, A. (2015). Fungal melanin: What do we know about structure? Frontiers in Microbiology, 6, 1463. https://doi.org/10.3389/fmicb. 2015.01463.

Passos, C. S., Simoes-Pires, C. A., Nurisso, A., Soldi, T. C., Kato, L., de Oliveira, C. M., .. Henriques, A. T. (2013). Indole alkaloids of Psychotria as multifunctional cholinesterases and monoamine oxidases inhibitors. Phytochemistry, 86, 8-20. https://doi. org/10.1016/j.phytochem.2012.11.015.

Ribera, J., Panzarasa, G., Stobbe, A., Osypova, A., Rupper, P., Klose, D., \& Schwarze, F. (2019). Scalable Biosynthesis of Melanin by the Basidiomycete Armillaria cepistipes. Journal of Agricultural and Food Chemistry, 67(1), 132-139. https://doi.org/10.1021/ acs.jafc. 8 b05071.

Ricklin, D., Reis, E. S., \& Lambris, J. D. (2016). Complement in disease: A defence system turning offensive. Nature Reviews Nephrology, 12(7), 383-401. https://doi.org/10. 1038/nrneph.2016.70.

Roche. (2016). Cell proliferation kit I (MTT). Retrieved from https://www.sigmaaldrich. com/content/dam/sigma-aldrich/docs/Roche/Bulletin/1/11465007001bul.pdf (accessed Feb 6th 2020)

Rosas, A. L., MacGill, R. S., Nosanchuk, J. D., Kozel, T. R., \& Casadevall, A. (2002). Activation of the alternative complement pathway by fungal melanins. Clinical and Diagnostic Laboratory Immunology, 9(1), 144-148. https://doi.org/10.1128/cdli.9.1. 144-148.2002.

Scheffler, A. (2019). The wound healing properties of betulin from birch bark from bench to bedside. Planta Medica, 85(7), 524-527. https://doi.org/10.1055/a-0850-0224.

Seo, H. W., Hung, T. M., Na, M., Jung, H. J., Kim, J. C., Choi, J. S., ... Min, B. S. (2009). Steroids and triterpenes from the fruit bodies of Ganoderma lucidum and their anticomplement activity. Archives of Pharmacal Research, 32(11), 1573-1579. https:// doi.org/10.1007/s12272-009-2109-x.

Shao, C. L., Linington, R. G., Balunas, M. J., Centeno, A., Boudreau, P., Zhang, C., Gerwick, W. H. (2015). Bastimolide A, a potent antimalarial polyhydroxy macrolide from the marine cyanobacterium Okeania hirsuta. Journal of Organic Chemistry, 80(16), 7849-7855. https://doi.org/10.1021/acs.joc.5b01264.

Shikov, A. N., Pozharitskaya, O. N., Makarov, V. G., Wagner, H., Verpoorte, R., \& Heinrich, M. (2014). Medicinal plants of the Russian pharmacopoeia; their history and applications. Journal of Ethnopharmacology, 154(3), 481-536. https://doi.org/10 
1016/j.jep.2014.04.007

Shin, Y., Tamai, Y., \& Terazawa, M. (2000). Chemical constituents of Inonotus obliquus: A new triterpene, 33-hydroxy-8,24-dien-lanosta21,23-lactone from sclerotium. Eurasian journal of forest research, 1, 43-50.

Sholichin, M., Yamasaki, K., Kasai, R., \& Tanaka, O. (1980). ${ }^{13} \mathrm{C}$ nuclear magnetic resonance of lupane-type triterpenes, lupeol, betulin and betulinic acid. Chemical \& Pharmaceutical Bulletin, 28, 1006-1008.

SvarLifeScience. (2015). WIESLAB ${ }^{\circledR}$ Complement system screen. Retrieved from https:// cdn2.hubspot.net/hubfs/2441243/Product\%20Documents\%20/COMPL300\%20-\% 20COMPL300RUO/COMPL\%20300\%20RUO\%20LABEL-DOC-0028\%20v2\%200.pdf (accessed March 13th 2020).

Tao, J., Li, Y., Li, S., \& Li, H.-B. (2018). Plant foods for the prevention and management of colon cancer. Journal of Functional Foods, 42, 95-110. https://doi.org/10.1016/j.jff. 2017.12.064.

Wang, C., Santhanam, R. K., Gao, X., Chen, Z, Chen, Y., Wang, C., .. Chen, H. (2018). Preparation, characterization of polysaccharides fractions from Inonotus obliquus and their effects on $\alpha$-amylase, $\alpha$-glucosidase activity and $\mathrm{H} 2 \mathrm{O} 2$-induced oxidative damage in hepatic L02 cells. Journal of Functional Foods, 48, 179-189. https://doi. org/10.1016/j.jff.2018.07.024

Wangensteen, H., Duong, G. M., Alamgir, M., Sarder, M., Samuelsen, A. B., \& Malterud, K.
E. (2006). Biological activities of limonoids, catechins, procyanidins and extracts from Xylocarpus granatum. Natural Product Communications, 1(11), 985-990.

Wold, C. W., Kjeldsen, C., Corthay, A., Rise, F., Christensen, B. E., Duus, J. O., \& Inngjerdingen, K. T. (2018). Structural characterization of bioactive heteropolysaccharides from the medicinal fungus Inonotus obliquus (Chaga). Carbohydrate Polymers, 185, 27-40. https://doi.org/10.1016/j.carbpol.2017.12.041.

Xu, Q., Zhao, Y., \& Zheng, W. (2015). Inonotus obliquus strain ATCC 28281 putative water-soluble melanin synthase gene, complete cds. Retrieved from https://www. ncbi.nlm.nih.gov/nuccore/KR069058.1 (accessed Feb 6th, 2020).

Yong, T., Chen, S., Liang, D., Zuo, D., Diao, X., Deng, C., ... Chen, D. (2018). Actions of Inonotus obliquus against hyperuricemia through XOD and bioactives screened by molecular modeling. International Journal of Molecular Sciences, 19(10), https://doi. org/10.3390/ijms19103222.

Zhao, F., Mai, Q., Ma, J., Xu, M., Wang, X., Cui, T., ... Han, G. (2015). Triterpenoids from Inonotus obliquus and their antitumor activities. Fitoterapia, 101, 34-40. https://doi. org/10.1016/j.fitote.2014.12.005.

Zheng, W., Miao, K., Liu, Y., Zhao, Y., Zhang, M., Pan, S., \& Dai, Y. (2010). Chemical diversity of biologically active metabolites in the sclerotia of Inonotus obliquus and submerged culture strategies for up-regulating their production. Applied Microbiology and Biotechnology, 87(4), 1237-1254. https://doi.org/10.1007/s00253-010-2682-4. 


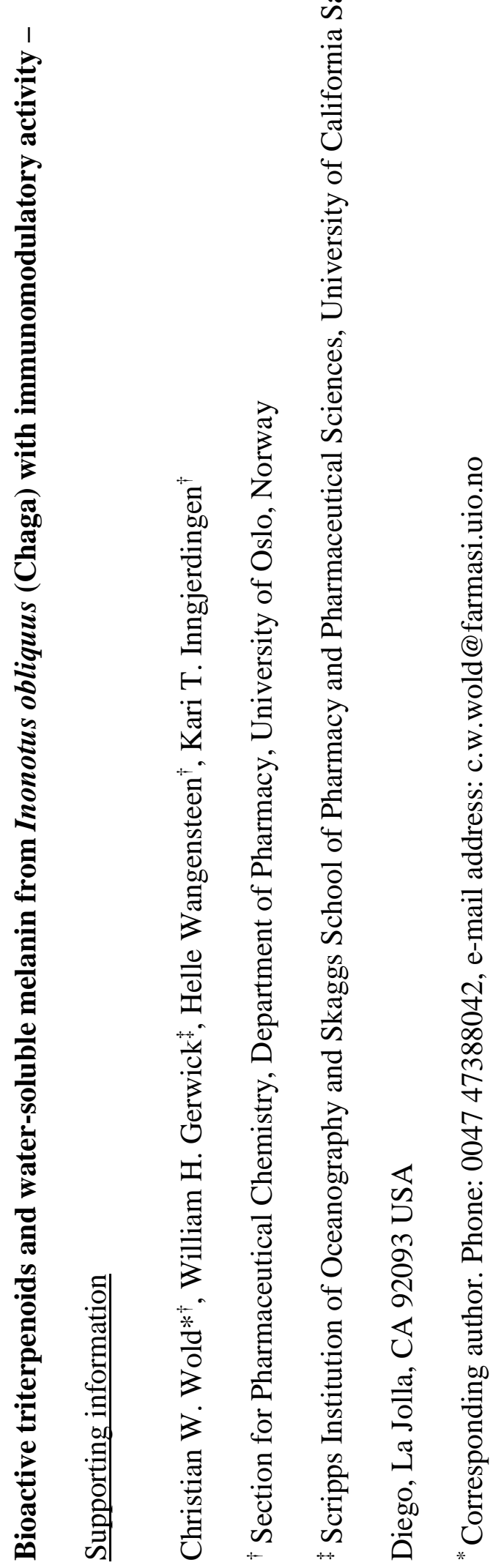




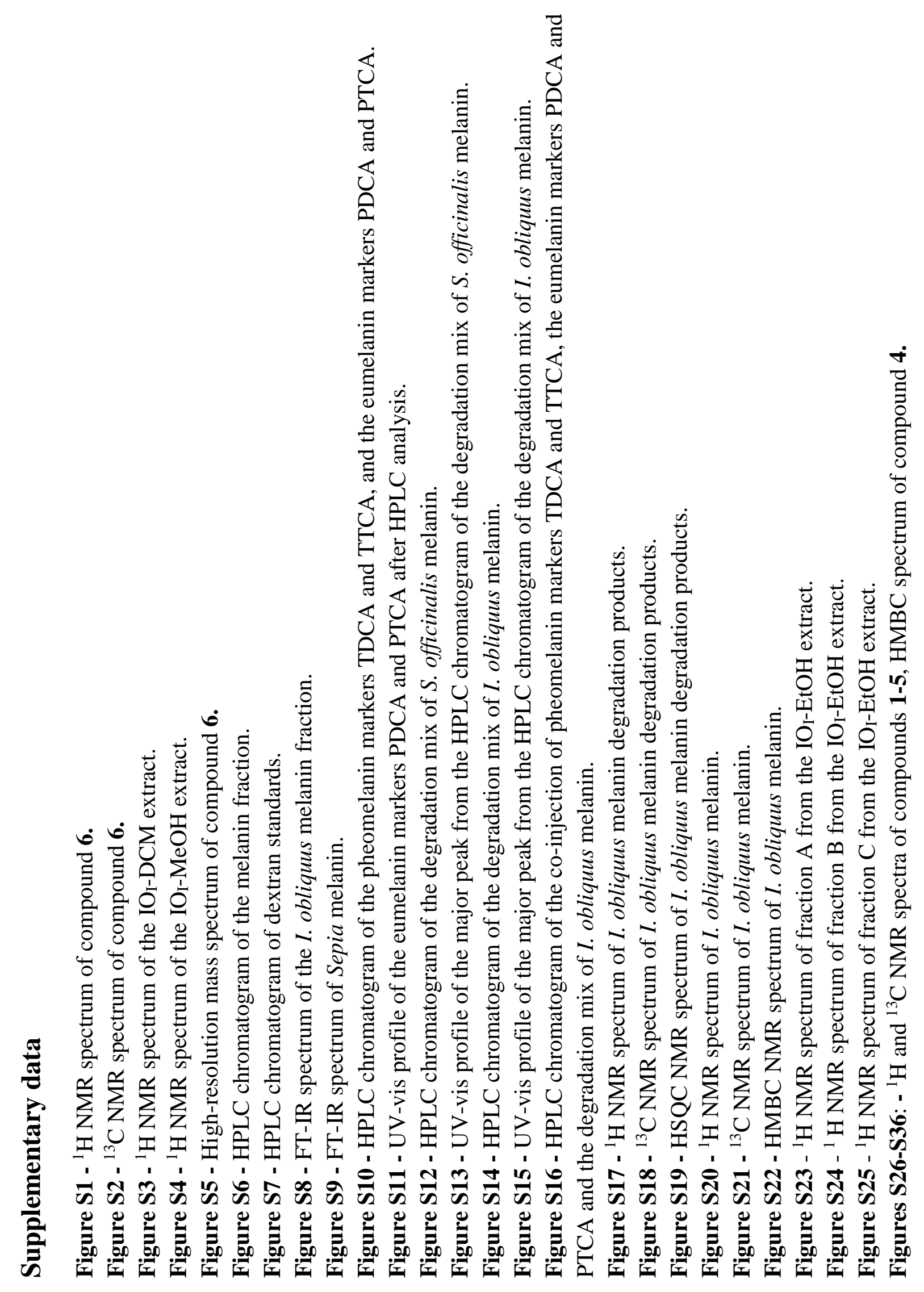














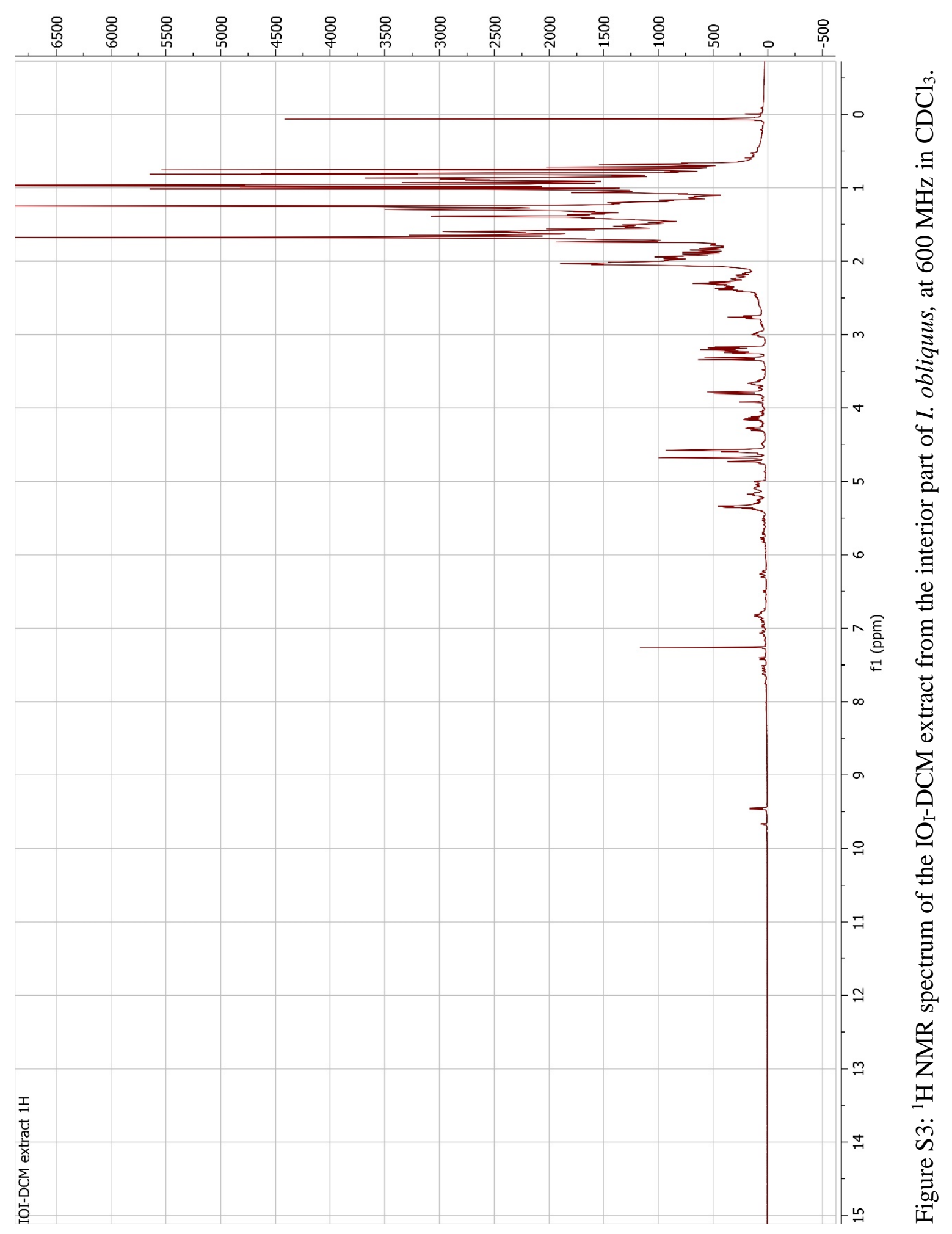









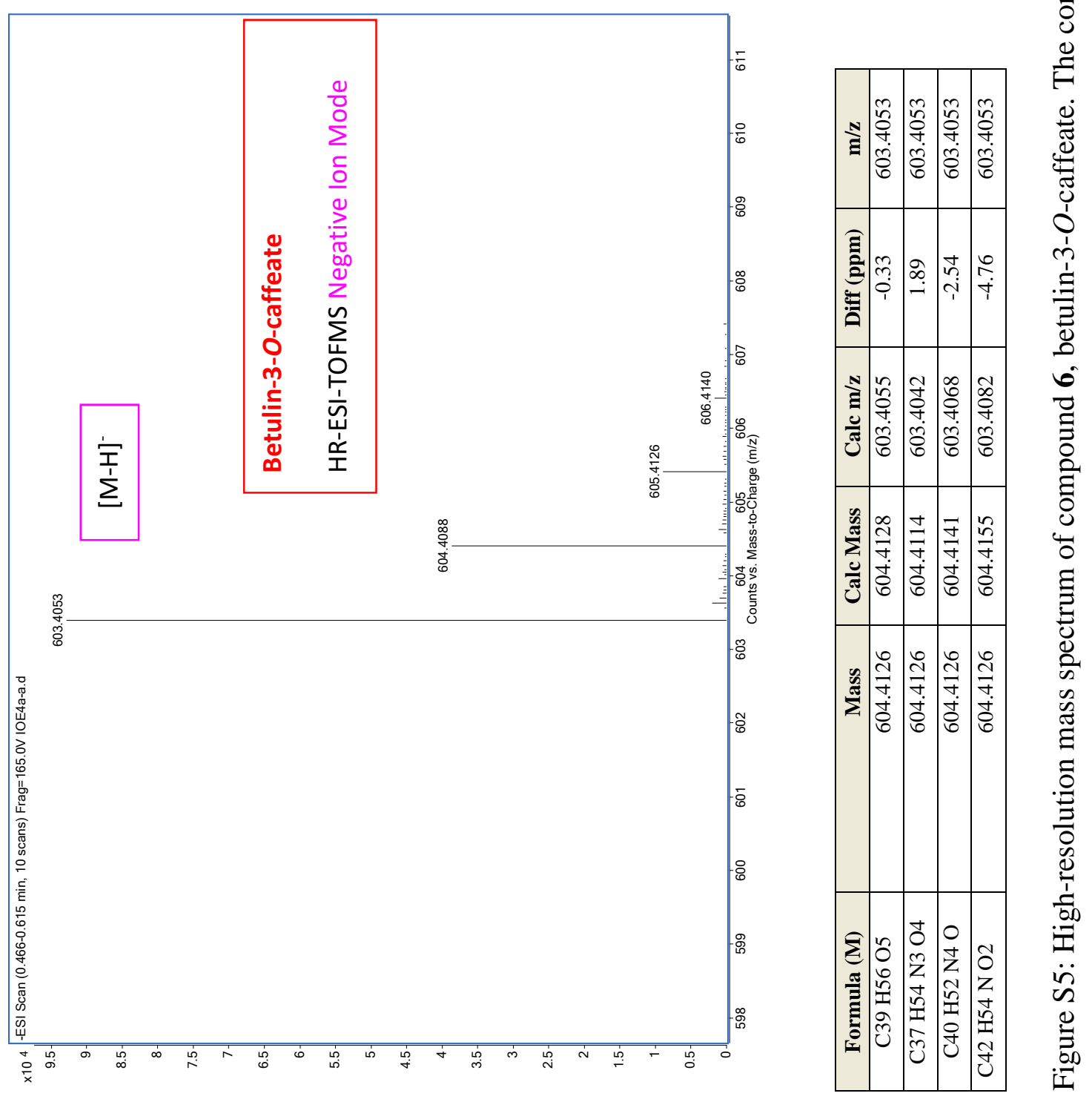


$\cap \forall m$

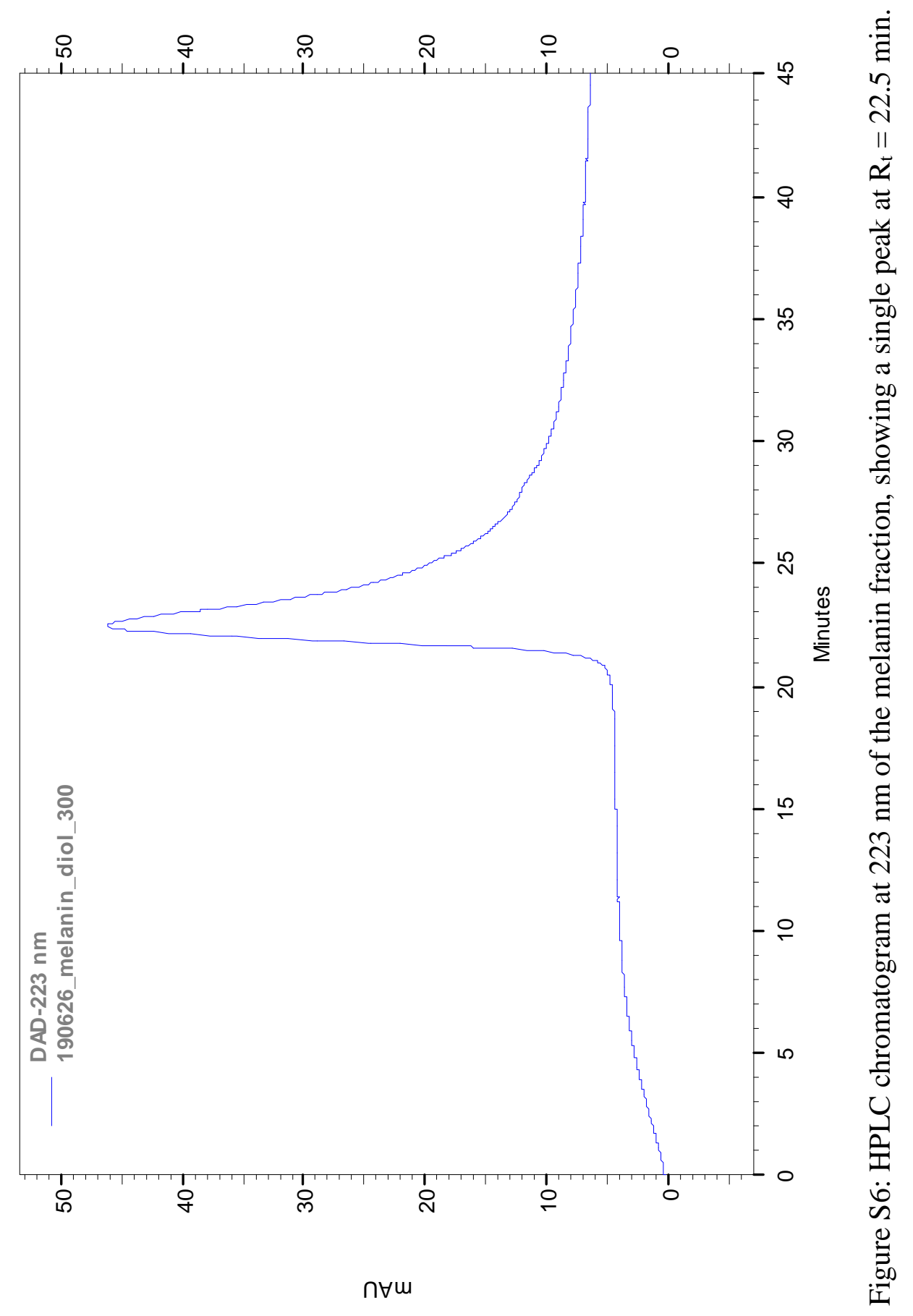




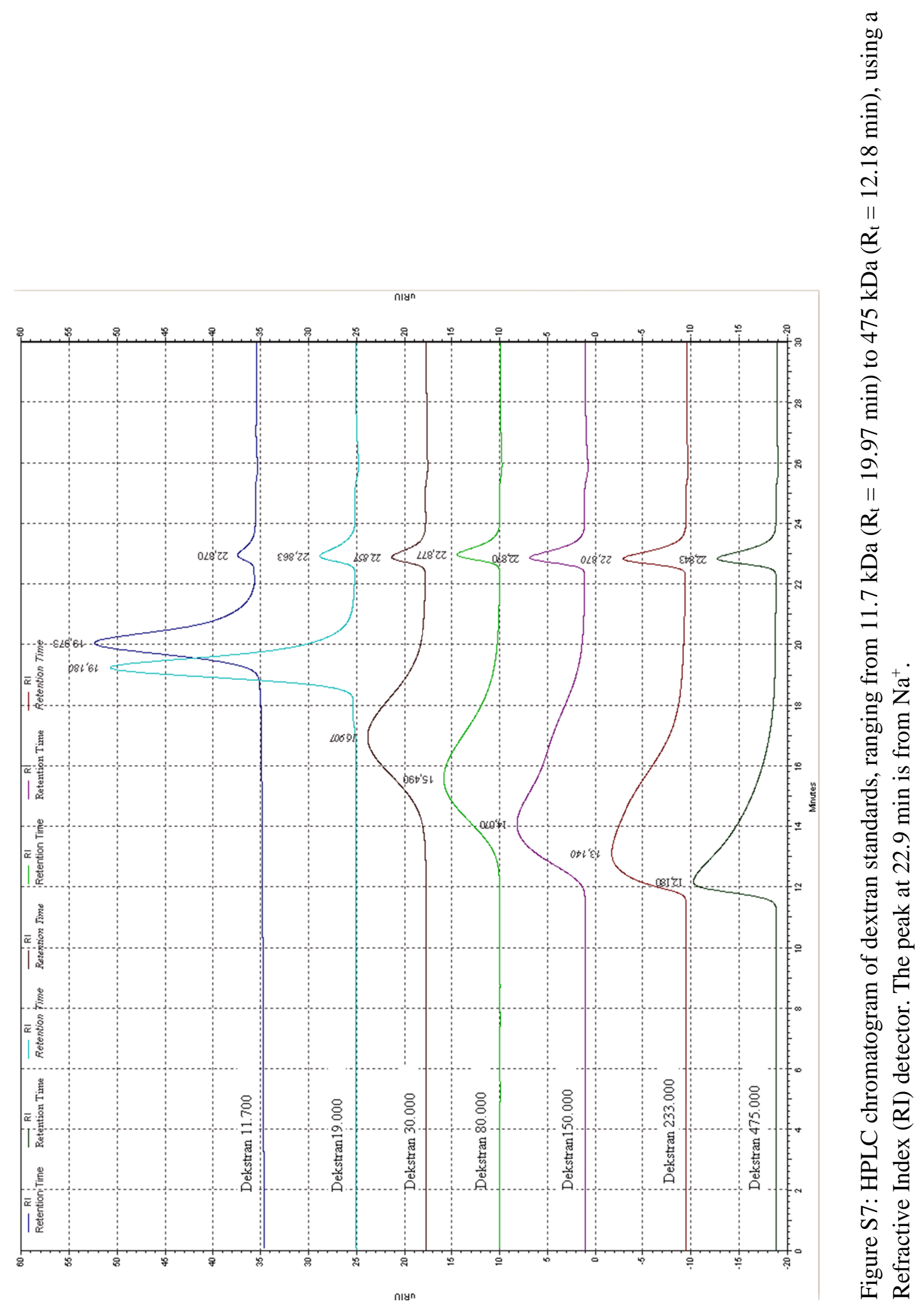




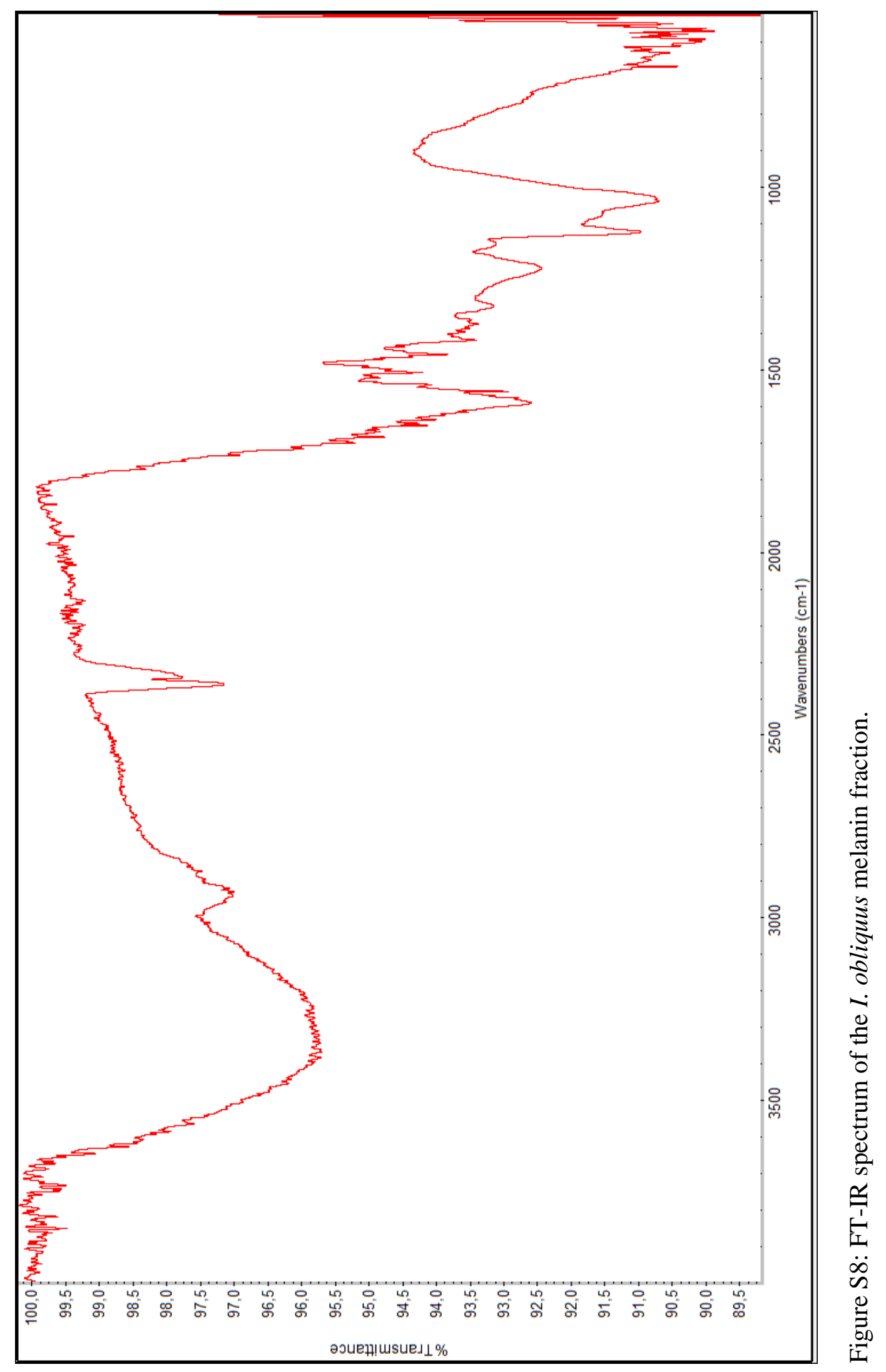




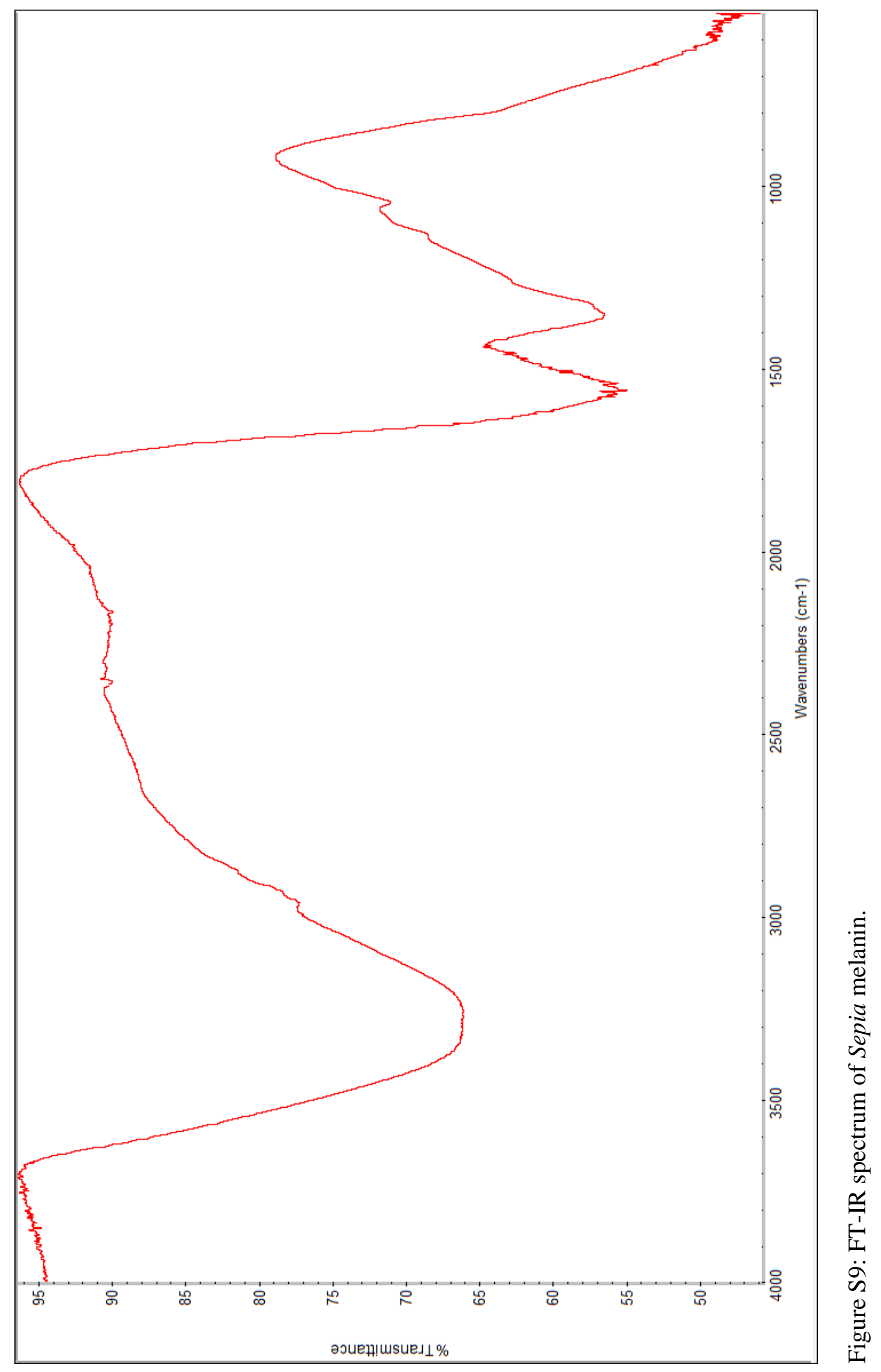




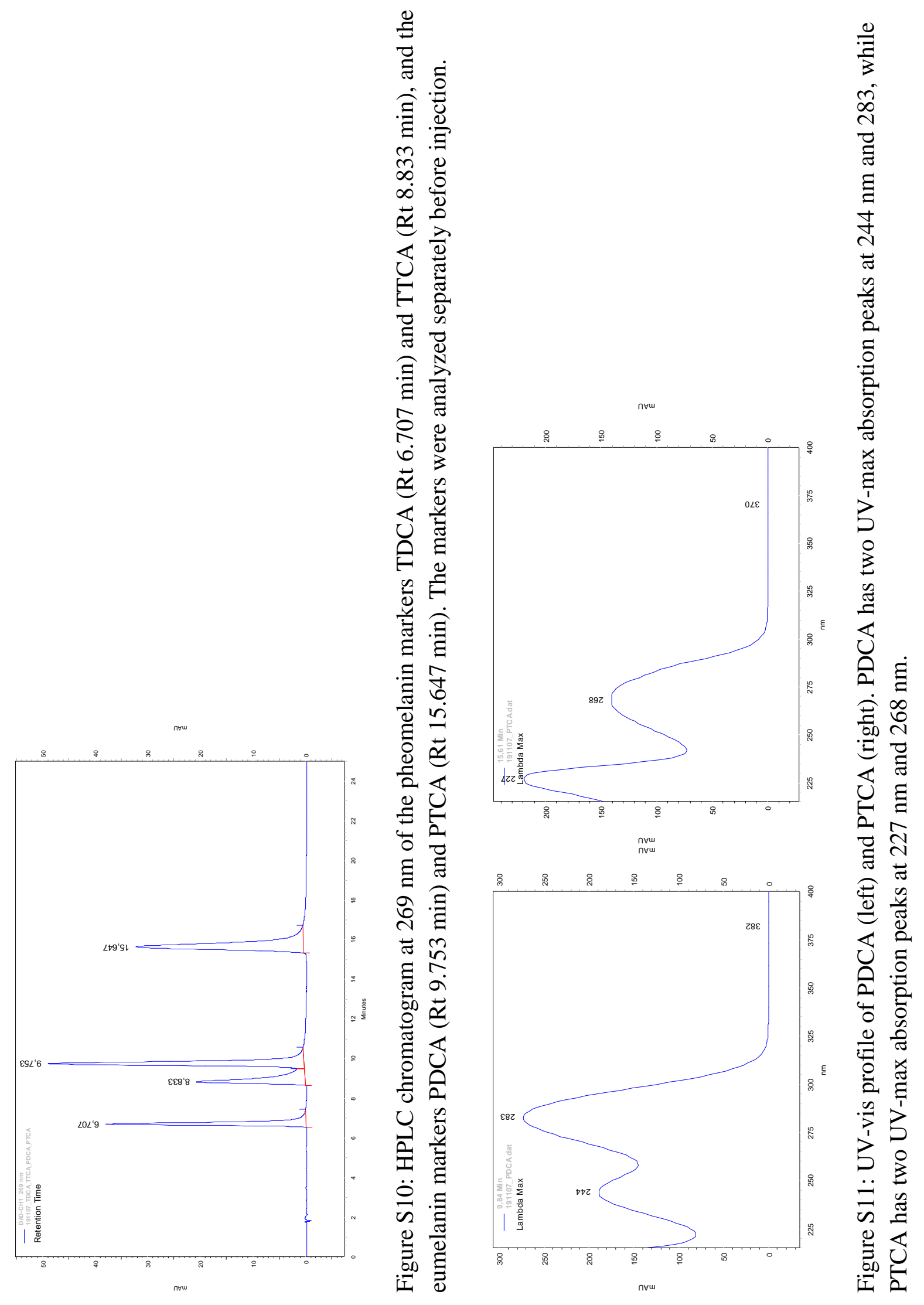




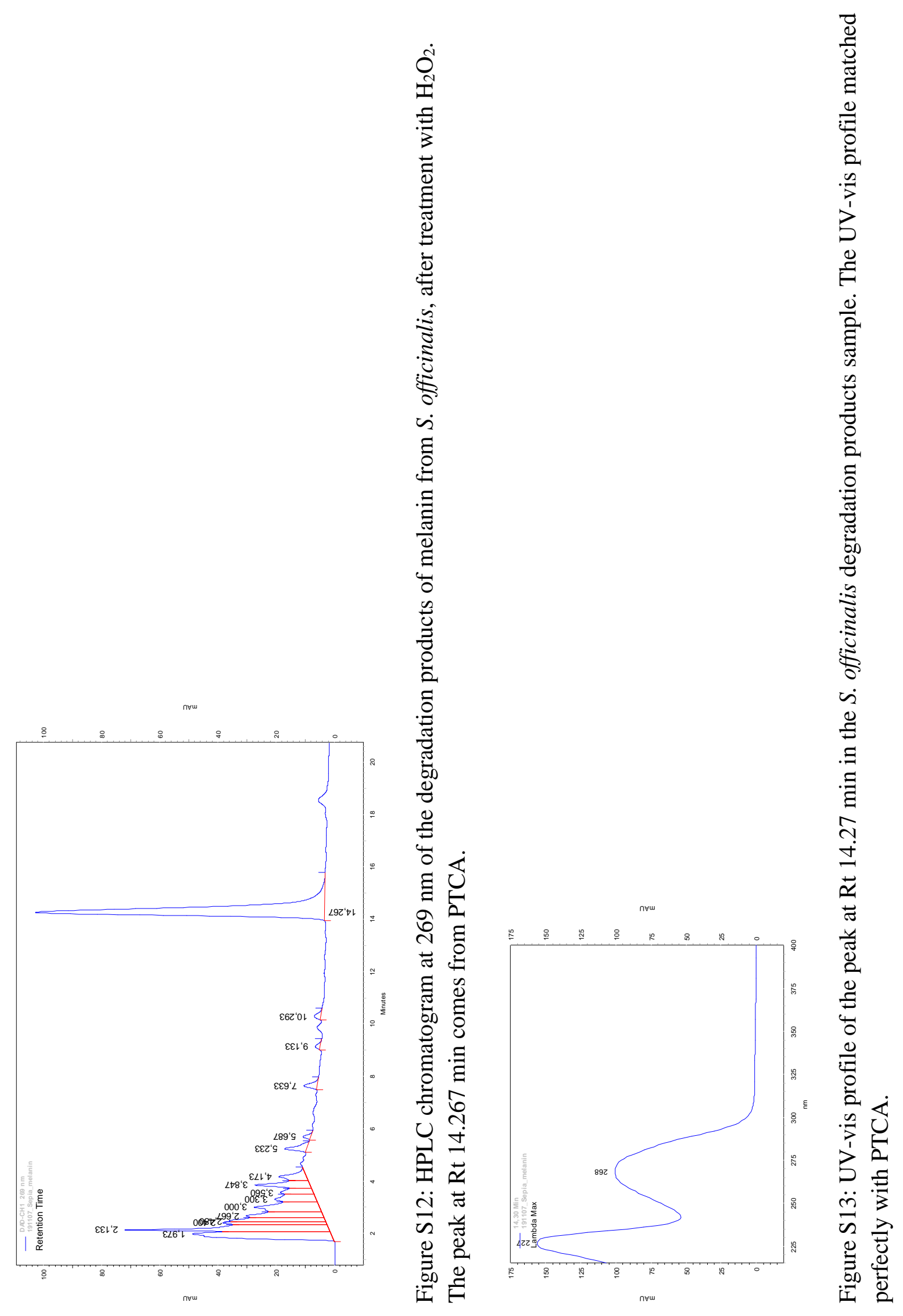




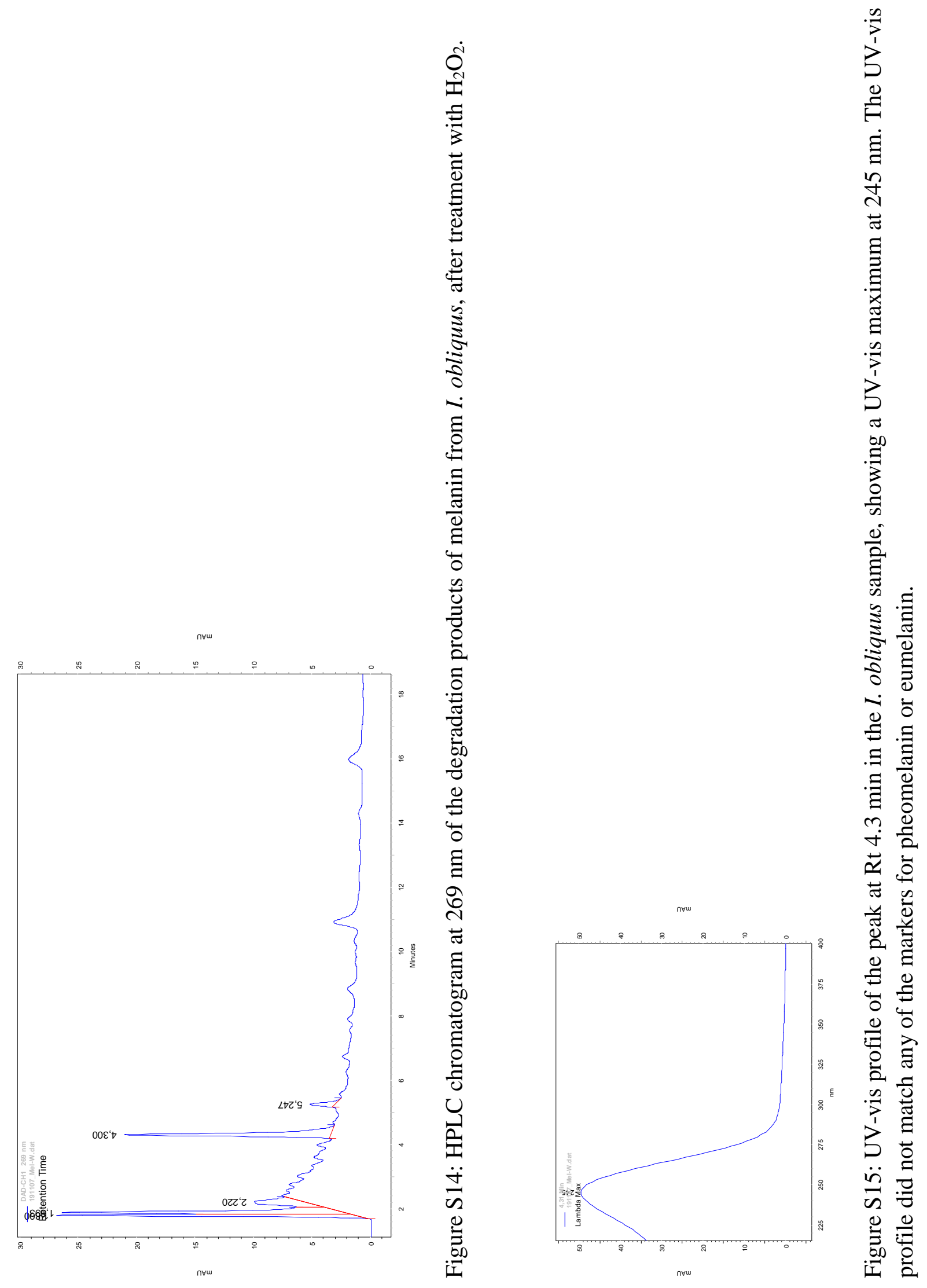




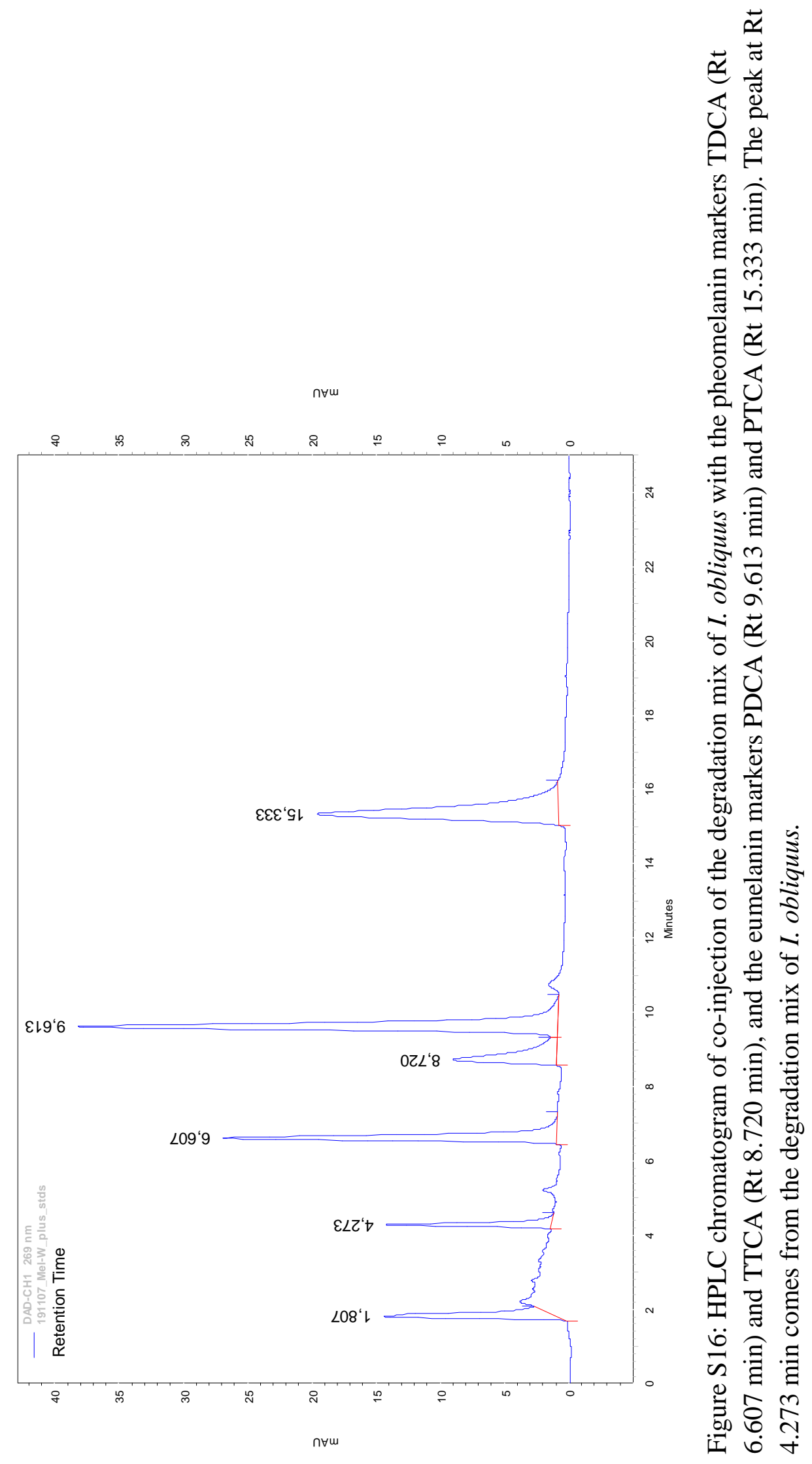




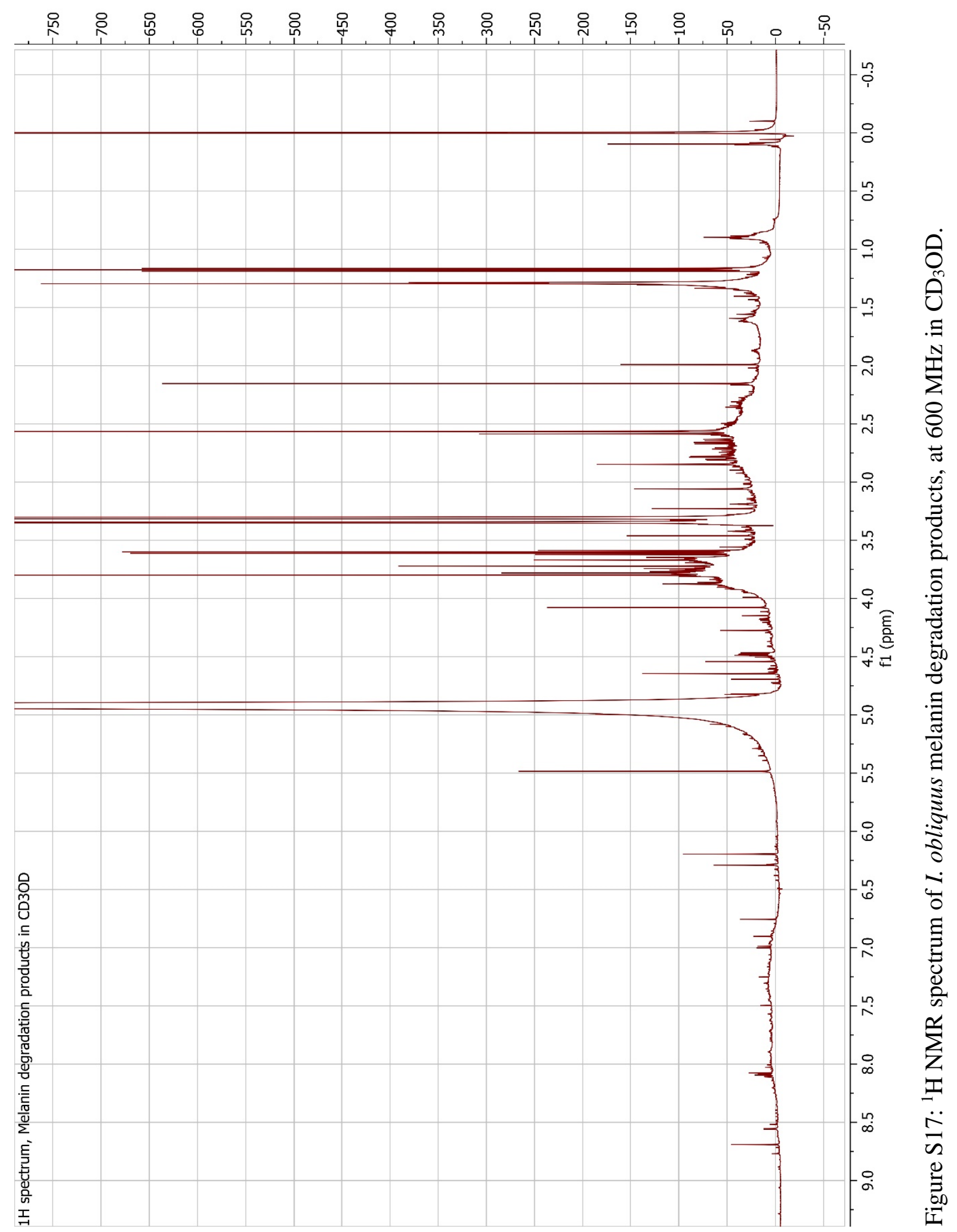




$$
1
$$


(udd) If

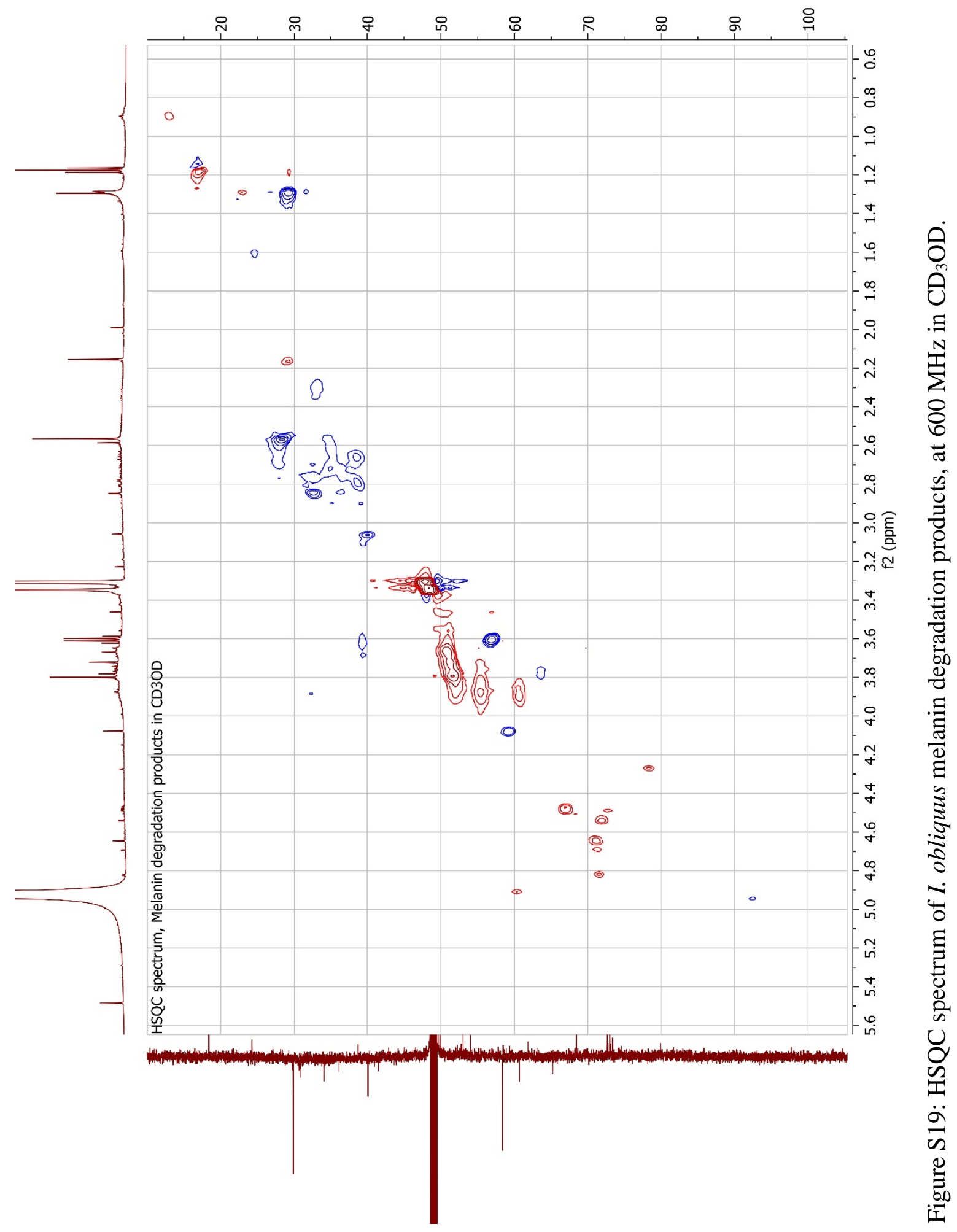




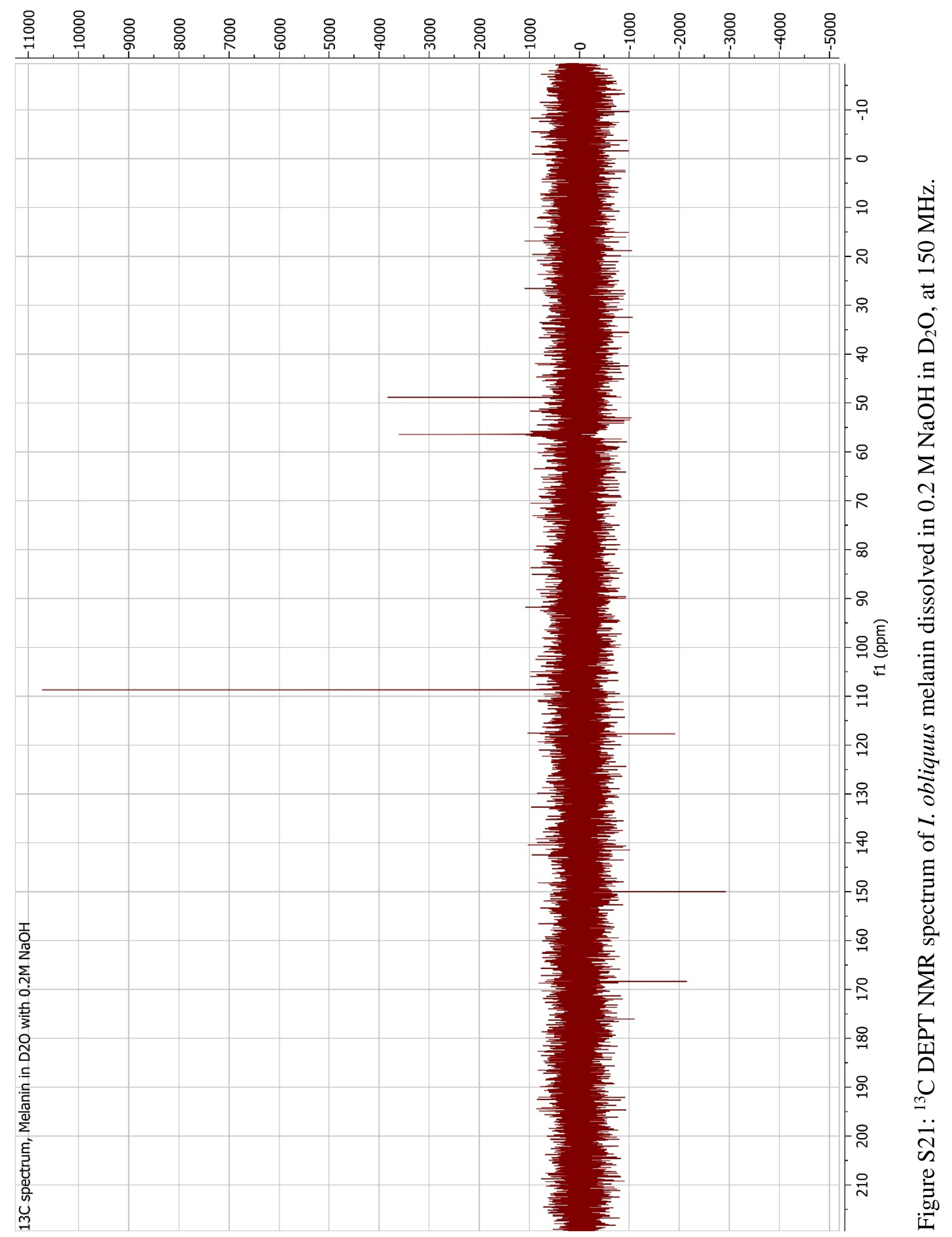


(udd) It

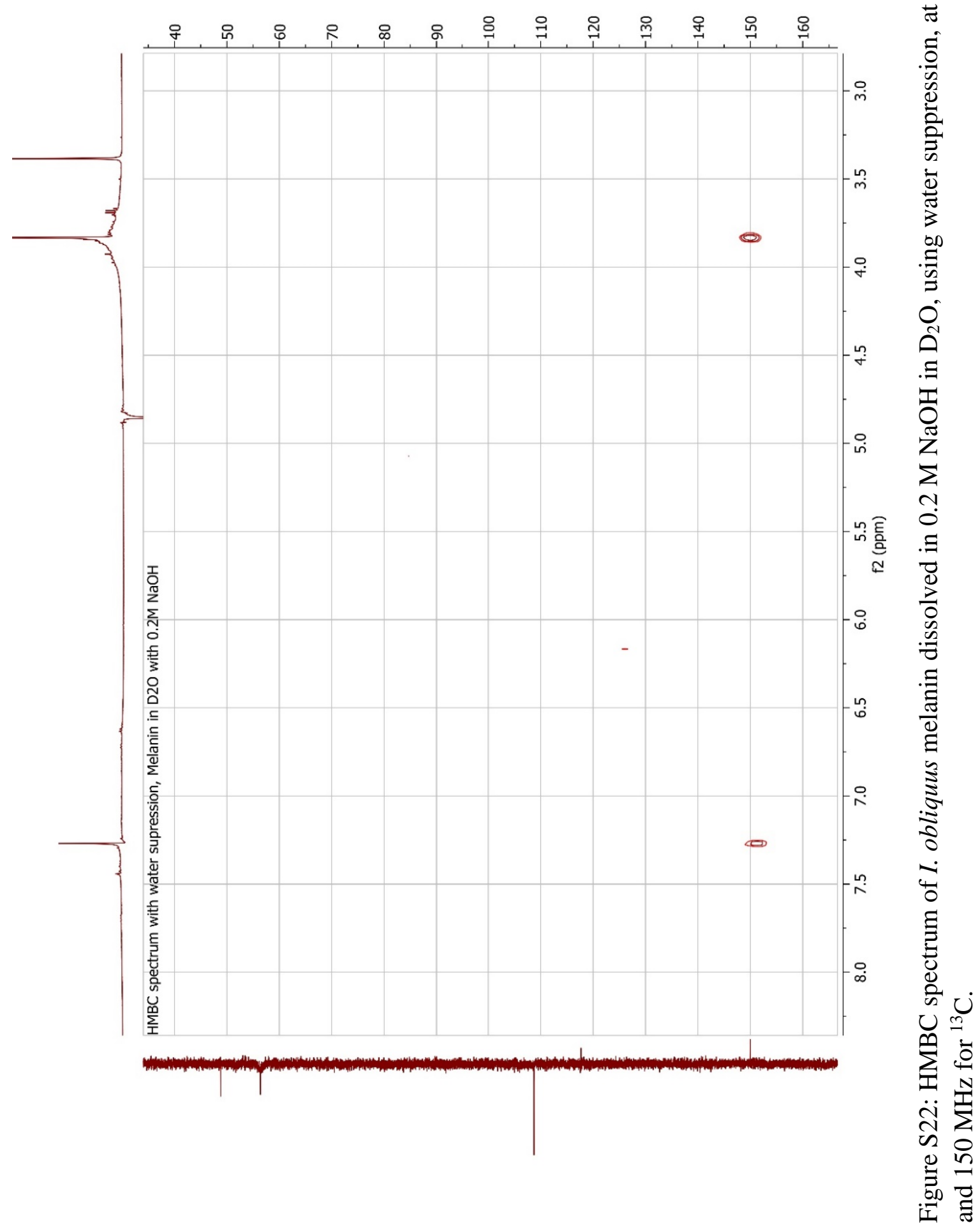




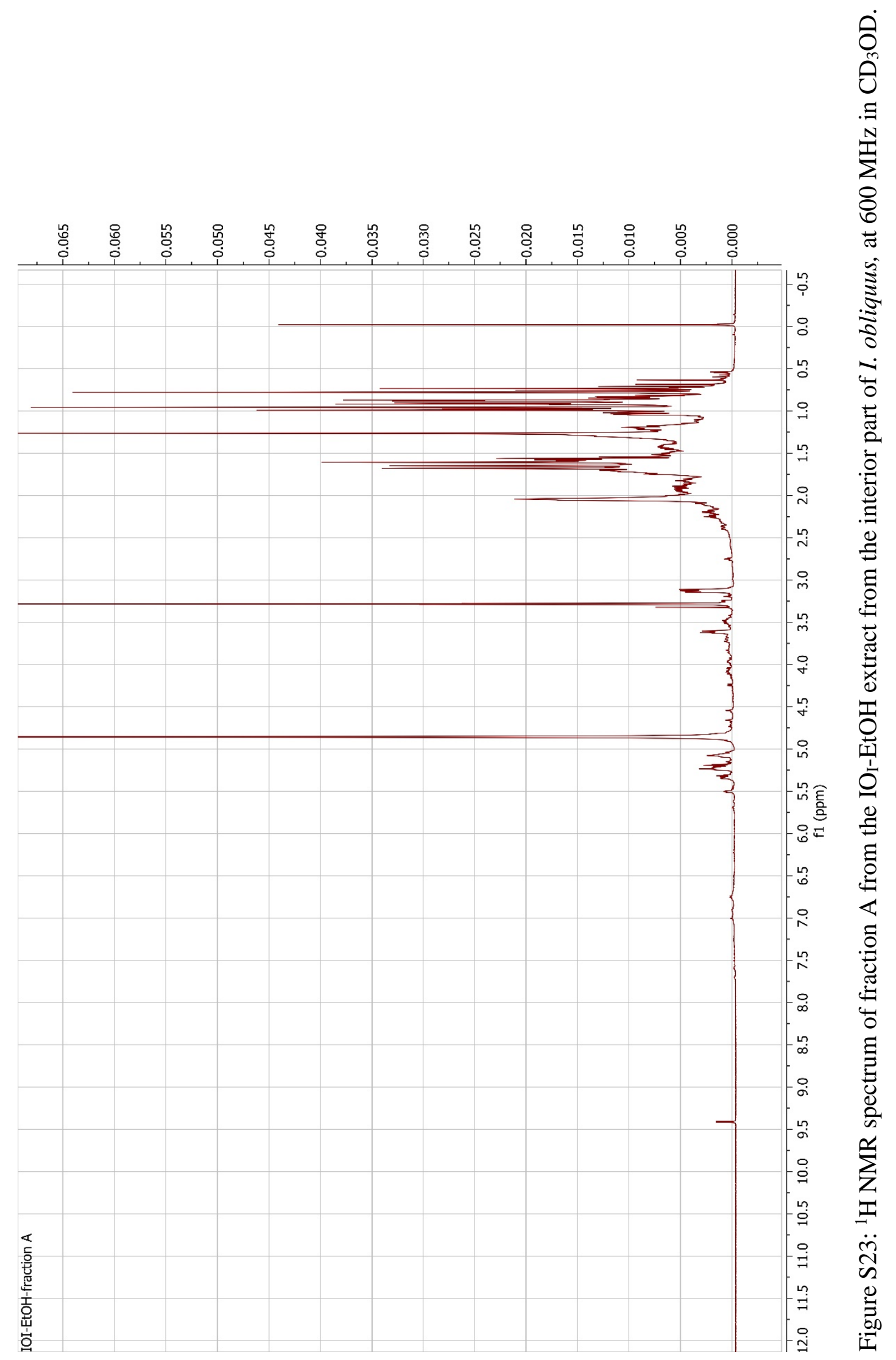




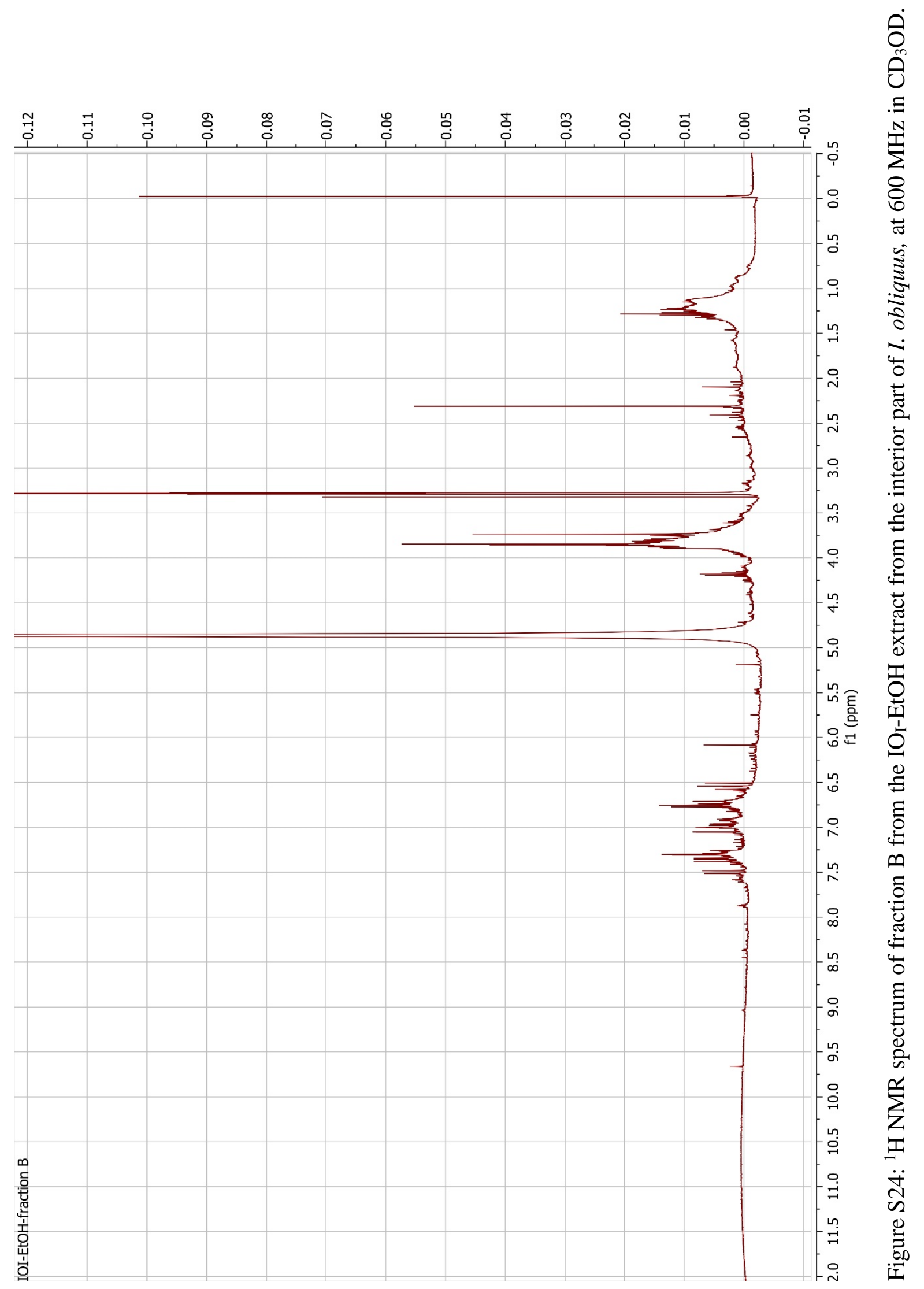




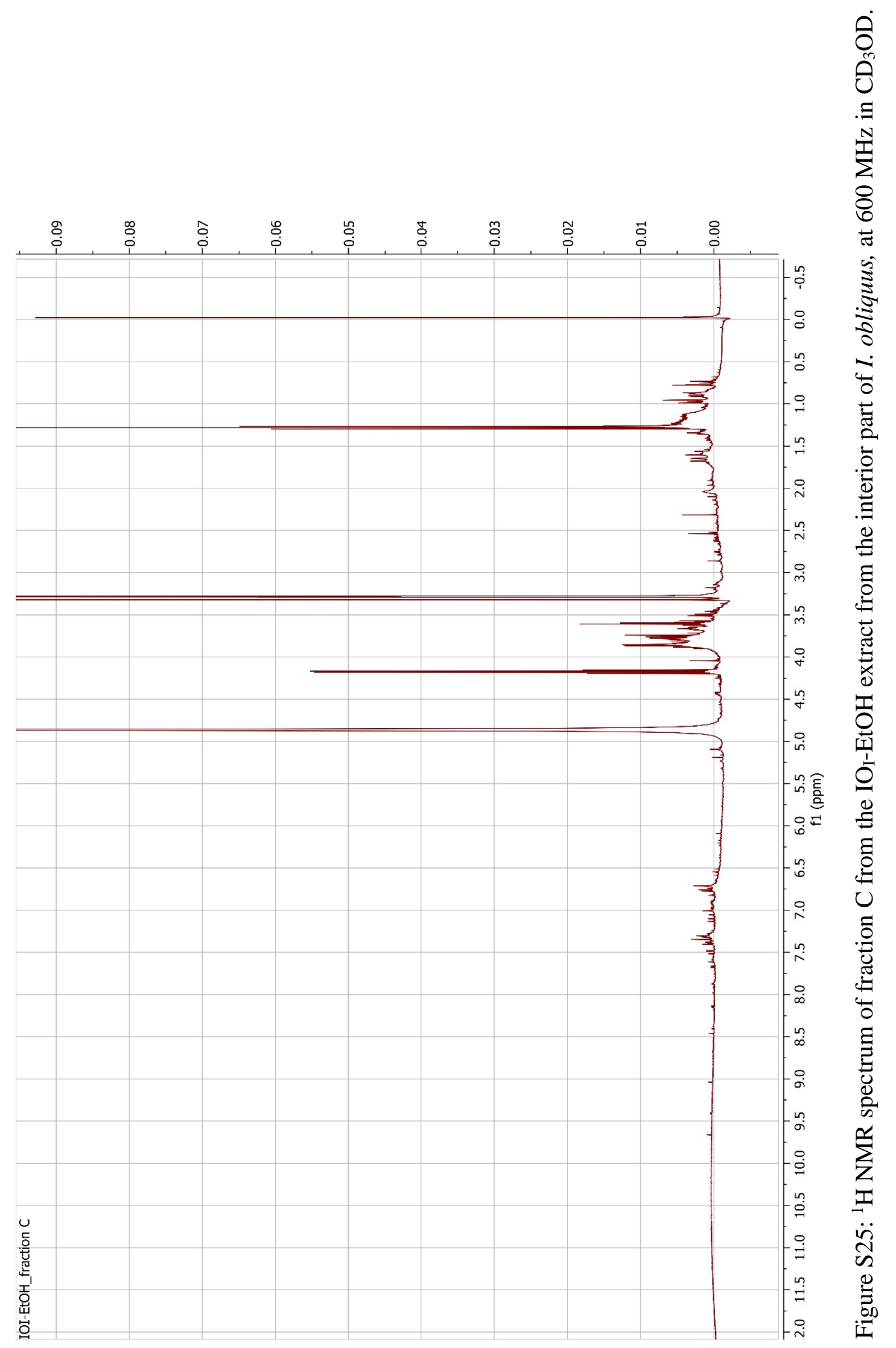









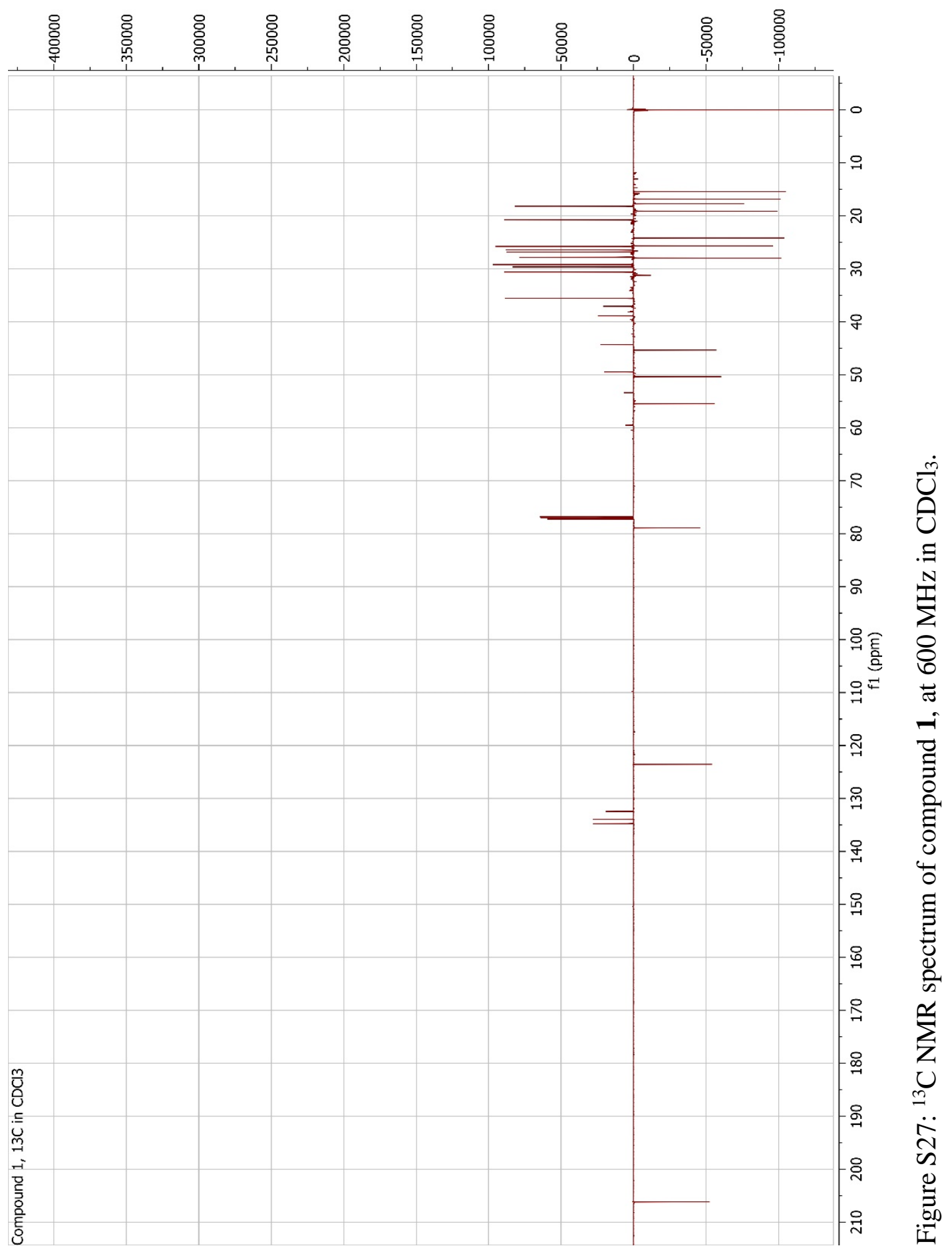




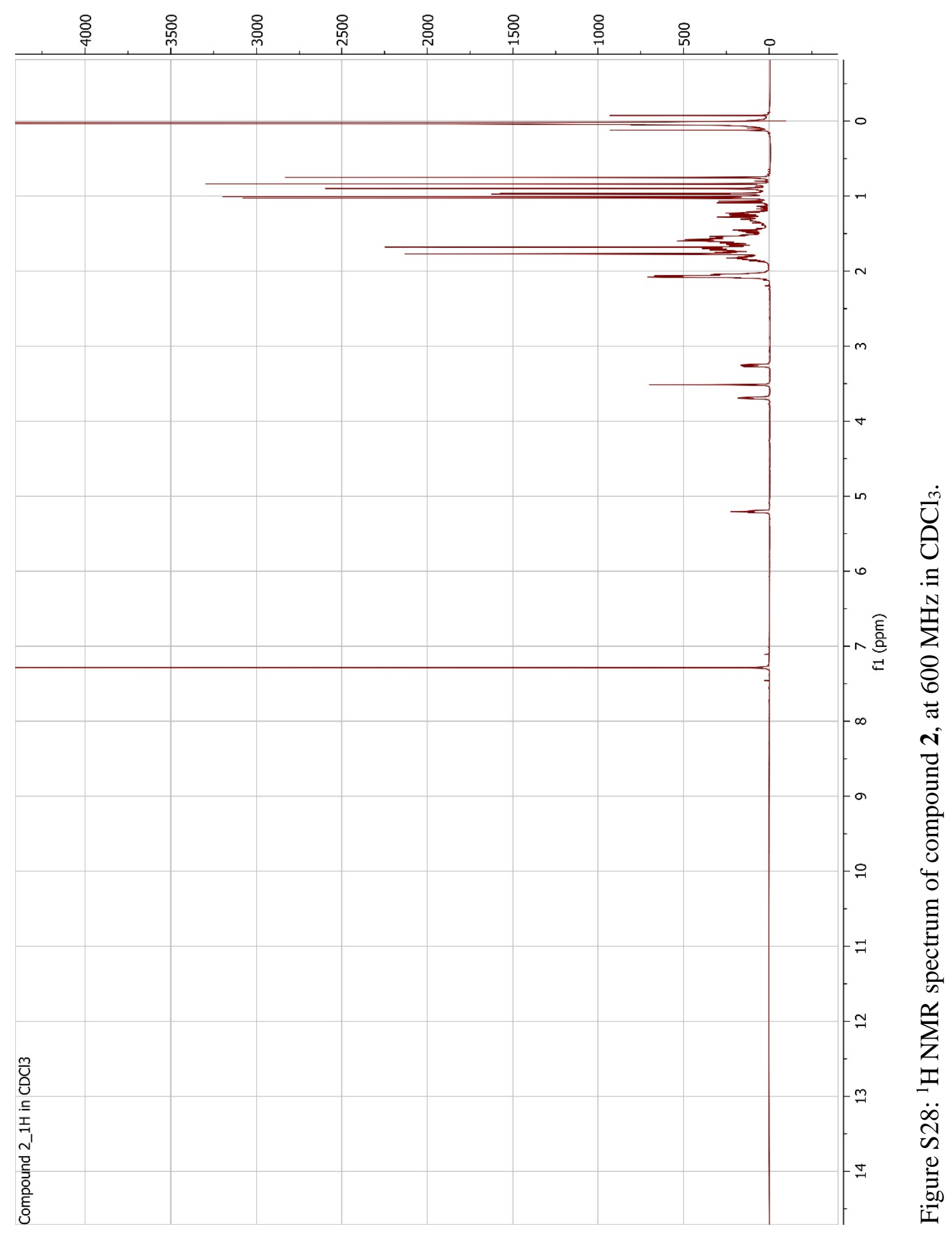




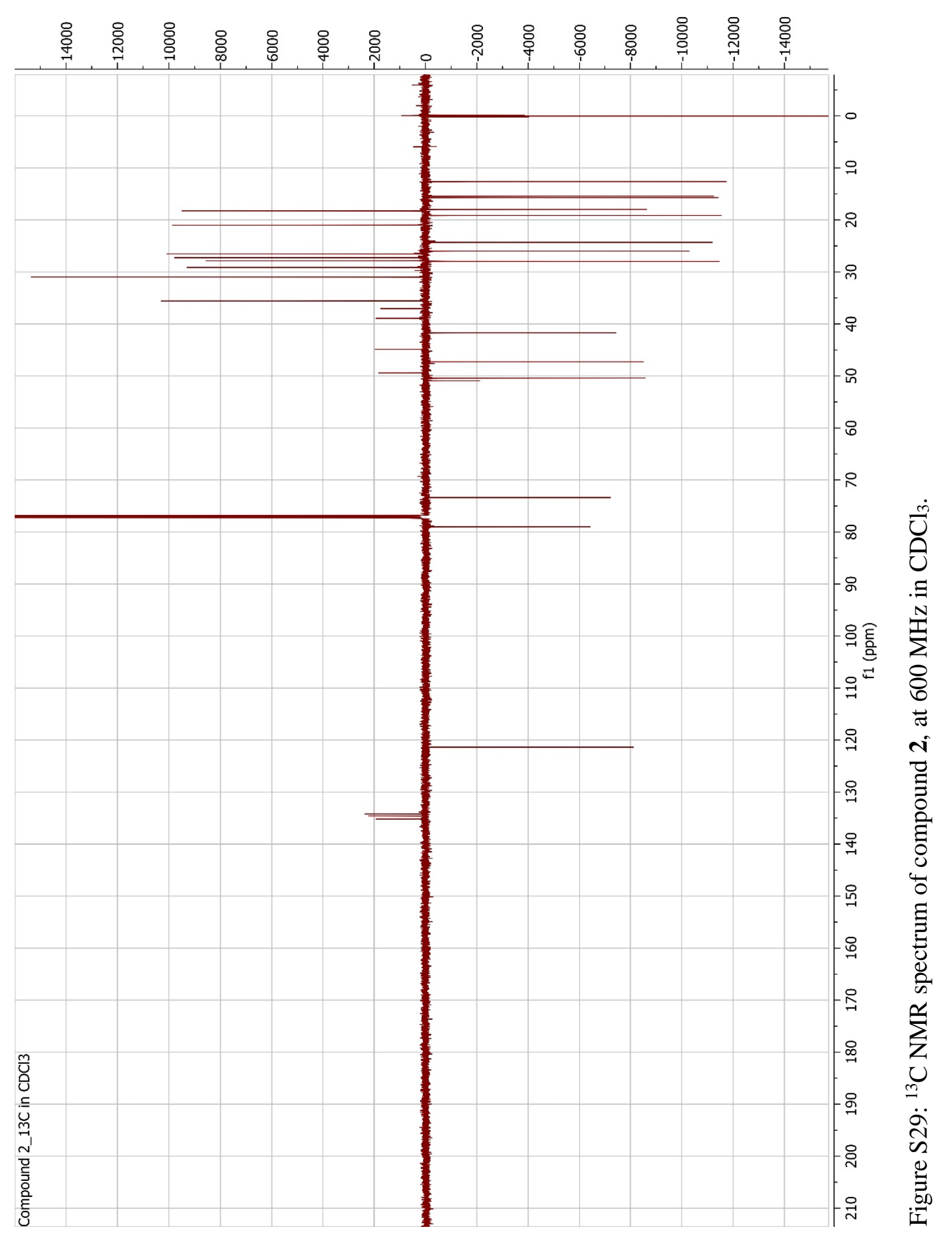




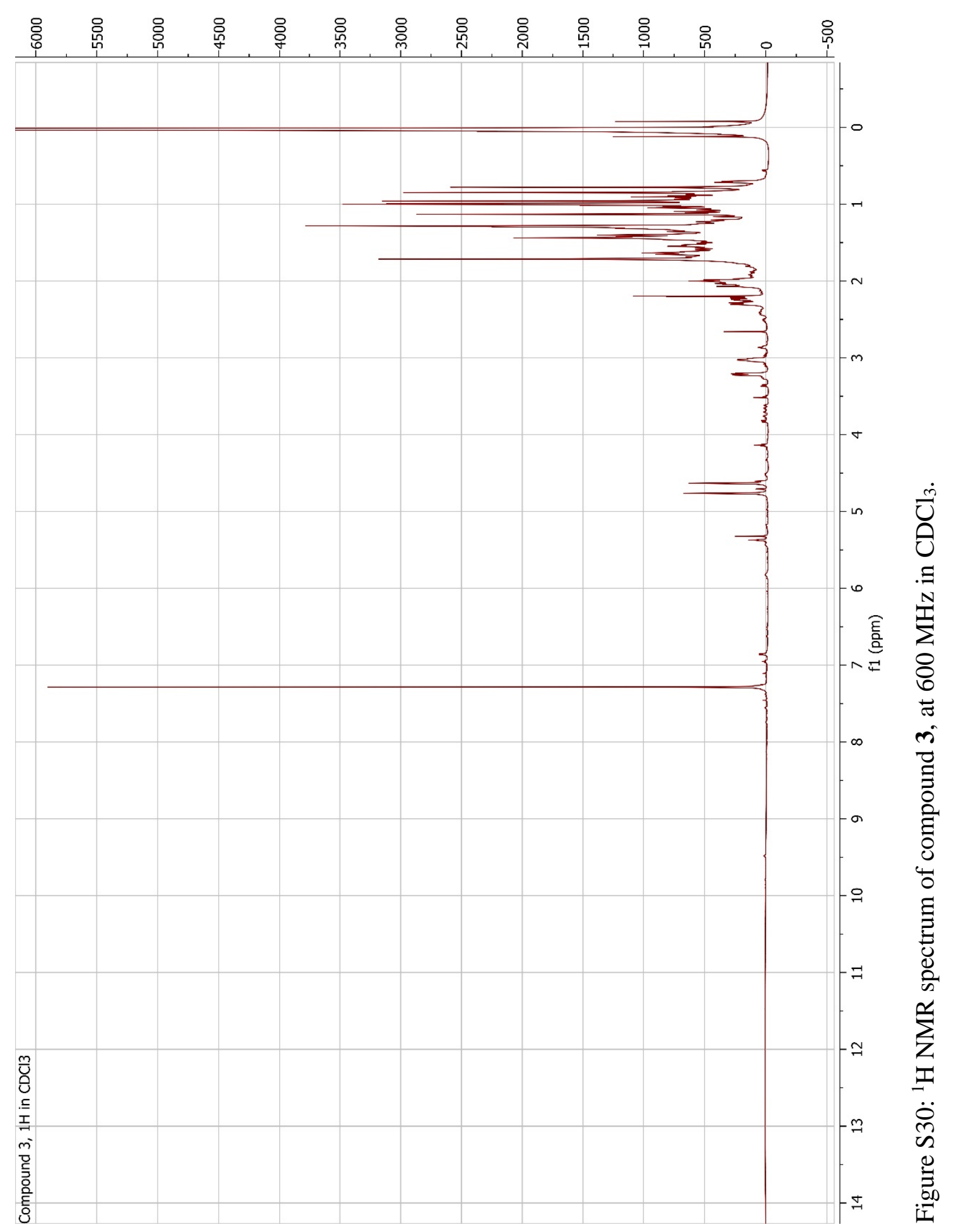




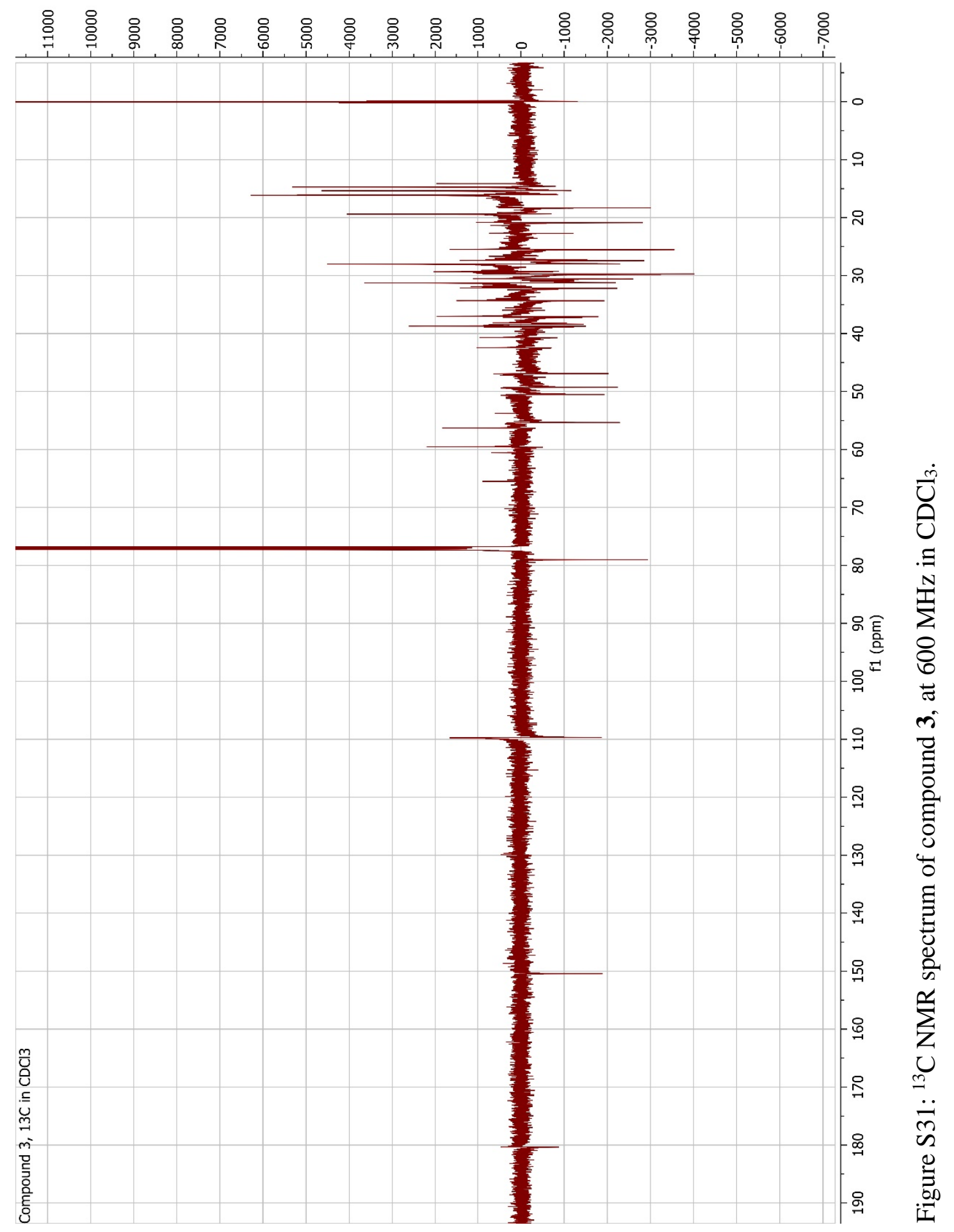




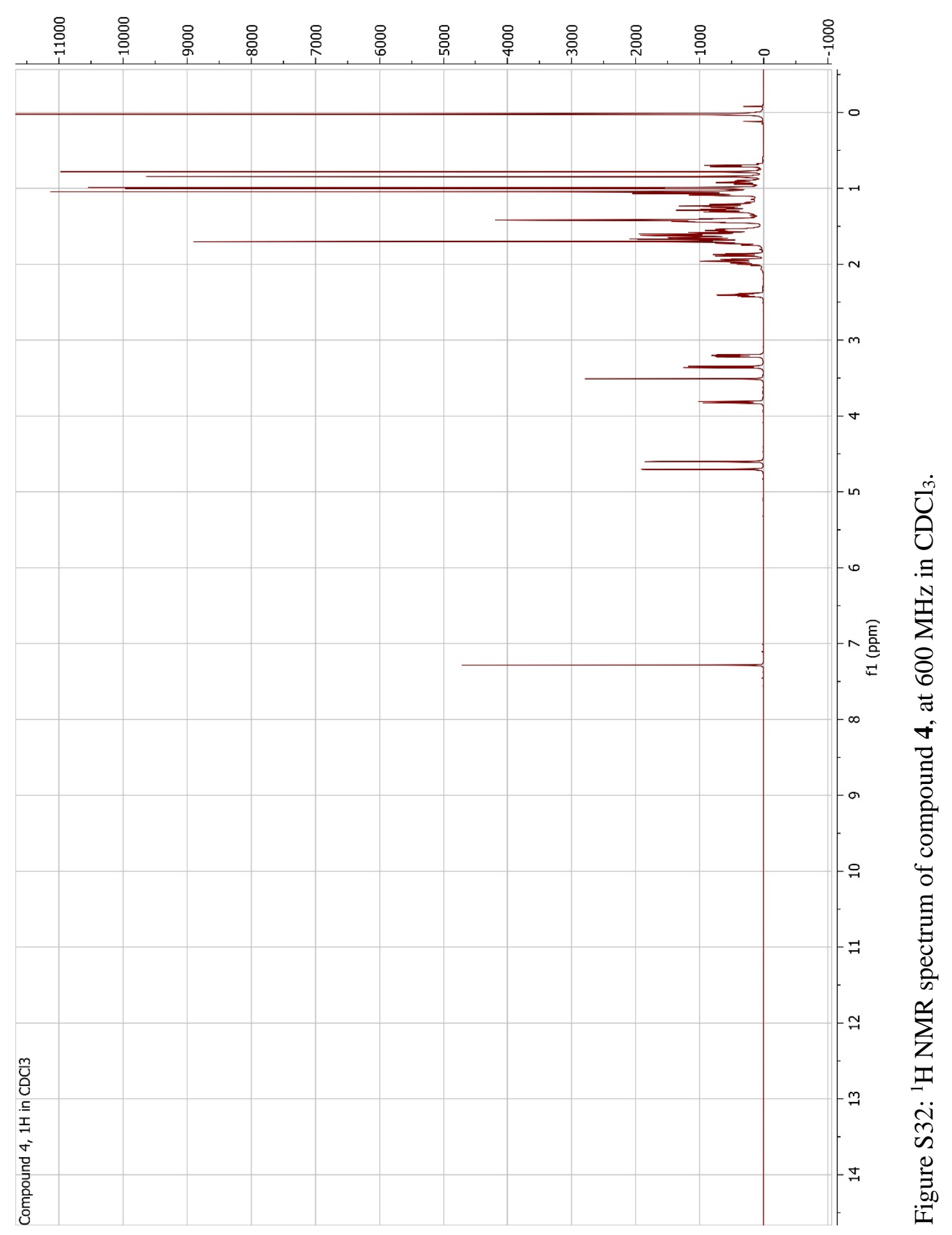




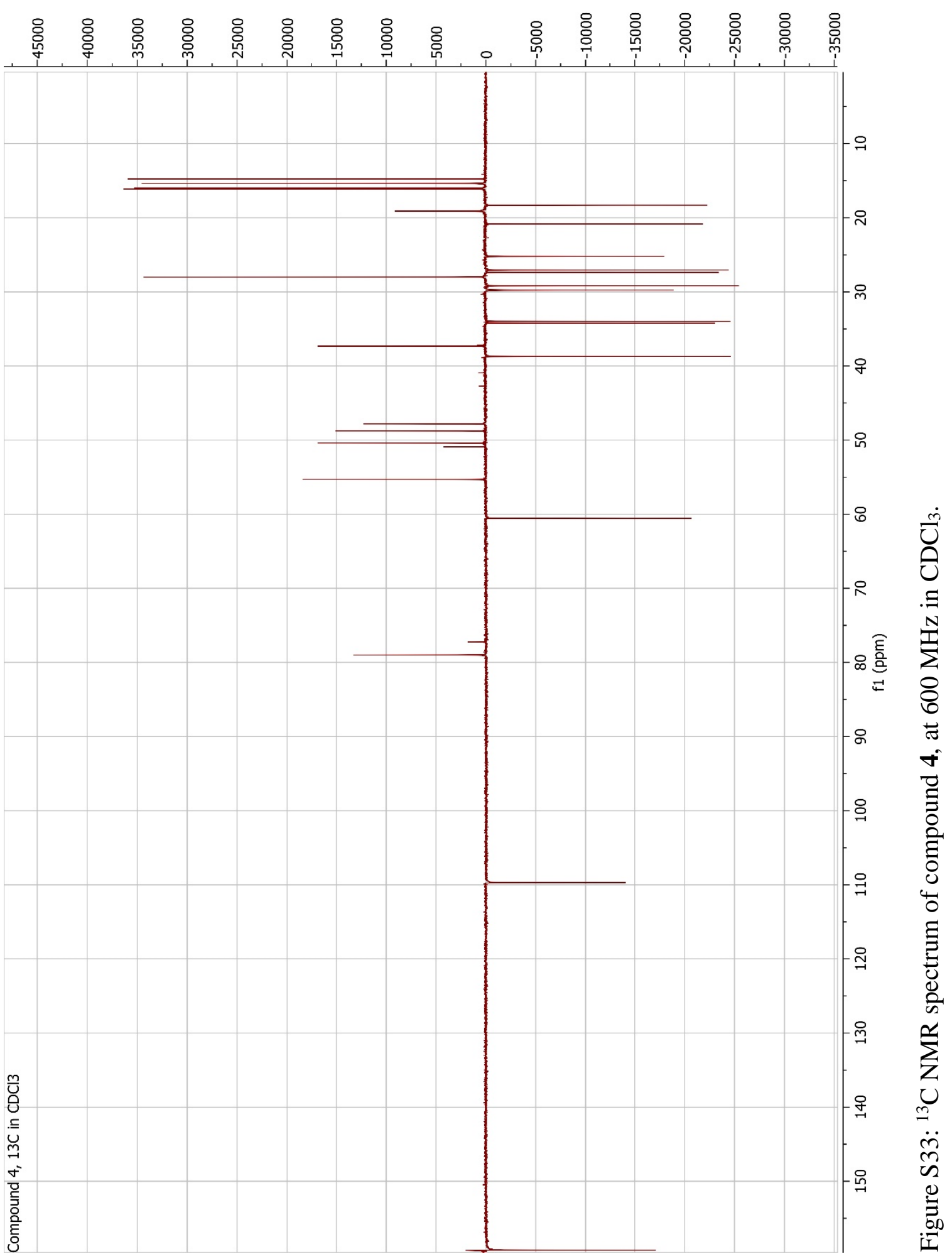


(urdd) If

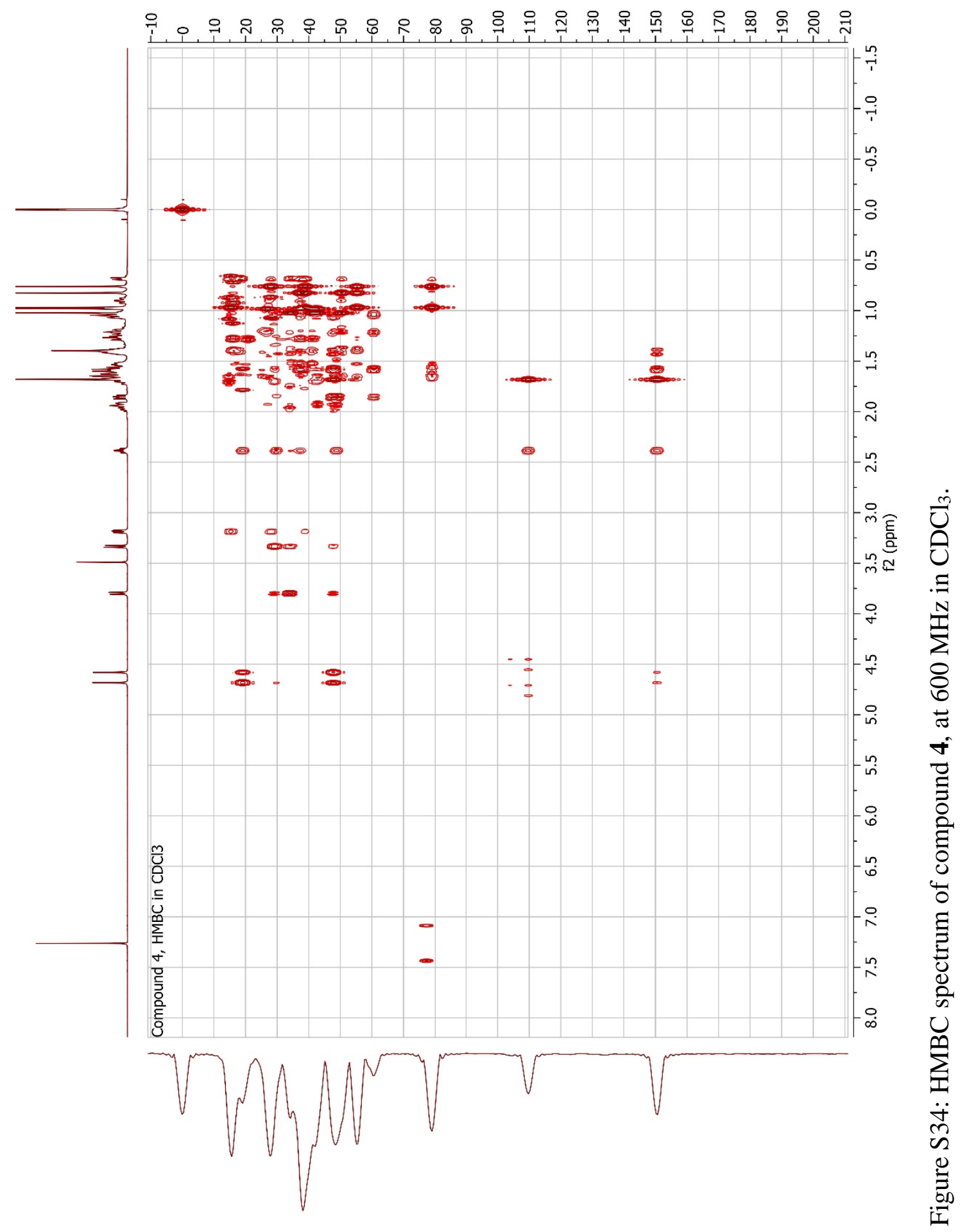




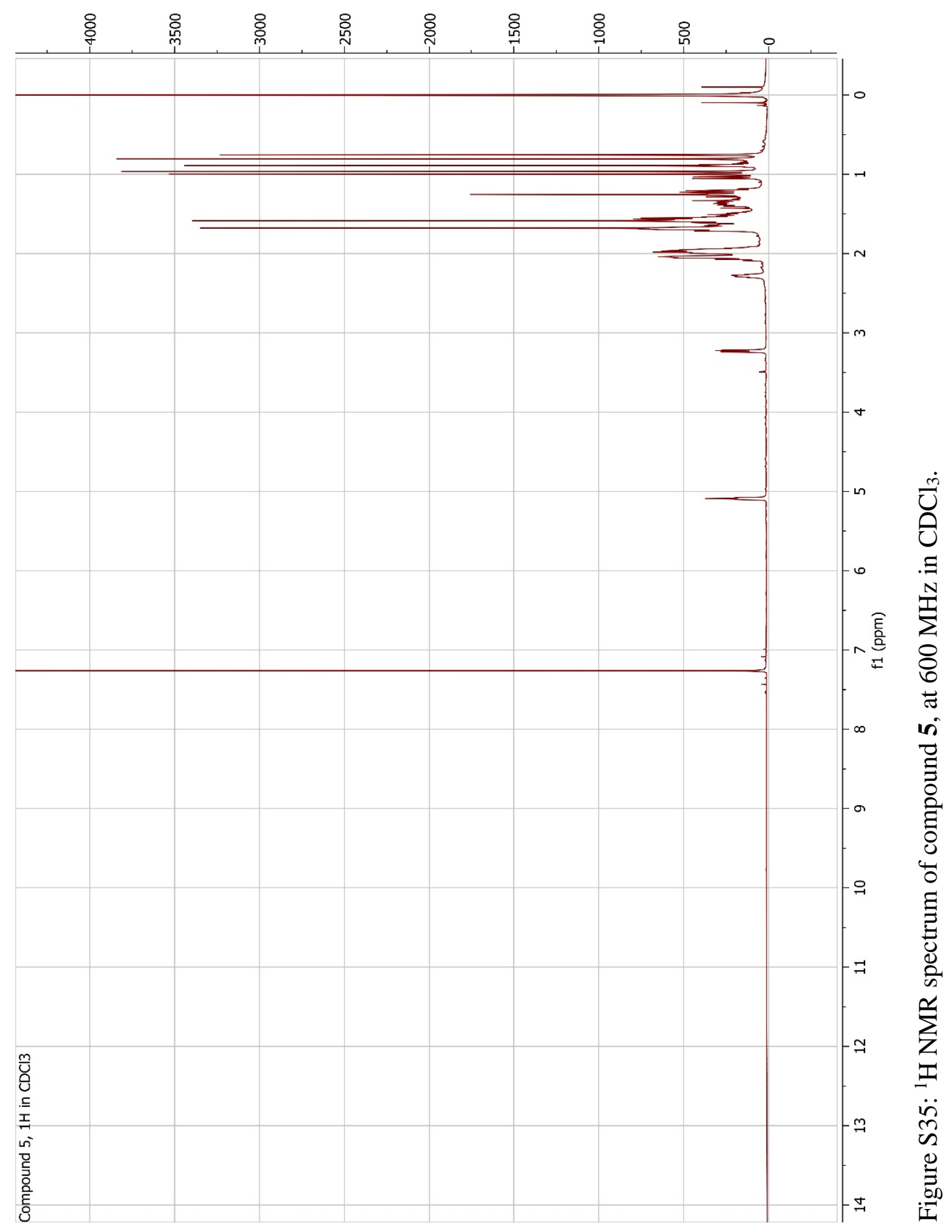




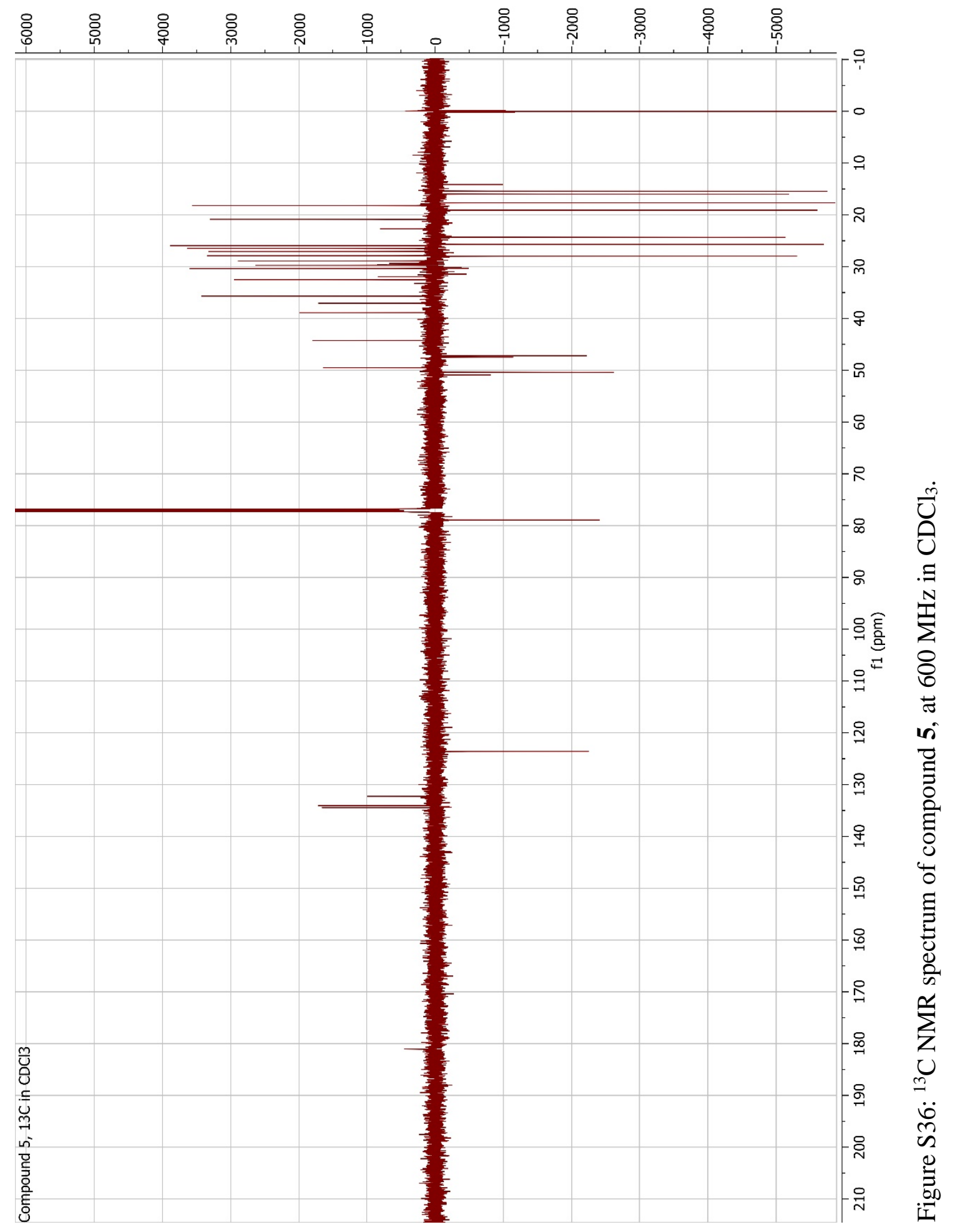

